\title{
RUMINATION IS A CORRELATE BUT NOT A CAUSE OF DEPRESSIVE SYMPTOMS DURING ADOLESCENCE
}

\author{
By \\ Kirsty Frances Weir
}

A thesis

Submitted to the Victoria University of Wellington in fulfillment of the requirements for the degree of

Doctor of Philosophy In Psychology

Victoria University of Wellington

2007 


\begin{abstract}
Data reported in this thesis were used to test the applicability of Response Styles Theory (RST; Nolen-Hoeksema, 1987; 1991) to preadolescent and adolescent populations across three studies. First, this thesis tested whether rumination maintained and exacerbated distress, and second, whether female adolescents ruminated more than male adolescents. In addition, this thesis compared rumination's ability to predict depression and anxiety with other predictors of maladjustment, such as stress, sense of control, and perceptions of false self. Study 1 had two major objectives, first to measure the concurrent relationships among stress, rumination, sense of control and depressive symptoms among 310 preadolescents (9-13 years). Results indicated that 13 year old females were slightly more ruminative and depressed than 13 year old males. Second, rumination mediated rather than moderated the relationship between stress and depressive symptoms. The second objective of Study 1 was to assess peer and parental influences in developing a ruminative response style. It was tested whether preadolescents expect females to ruminate more than males. In addition, parents ( $N$ $=218$ ) of these preadolescents reported whether they would encourage female children to ruminate more than male children. Data showed that preadolescents did not expect female preadolescents to ruminate more than male preadolescents, and similarly, parents did not encourage female children to ruminate more than male children.
\end{abstract}

Study 2 also had two objectives: first, to develop and validate a new scale entitled the Perceptions of False Self scale (POFS), enabling an additional predictor of maladjustment to be compared with rumination. This scale was constructed 
because existing scales (SWIT; Harter \& Waters, 1991) are difficult for adolescents to understand and complete. The POFS scale was developed using a multi-method approach, involving 331 adolescents (11-16 years). The POFS scale proved to be reliable and valid, and exhibited a stronger relationship with maladjustment (depression and anxiety) than existing measures. Study 2 also reported the relationships among stress, rumination, sense of control, perceptions of false self, and maladjustment among 195 adolescents across 10 weeks. Rumination predicted increases in later maladjustment when measured in isolation. However when stress, sense of control, and false self were included as predictors, rumination no longer predicted changes in maladjustment. Interestingly, the direction of effect among the measured variables seemed to flow from maladjustment to risk and vulnerability.

In Study 3, depressive and anxious symptoms were measured separately to test whether stress, rumination, sense of control, and perceptions of false self exhibited distinct relationships with anxious and depressive symptoms. Data were collected from 926 adolescents (11-17 years) across 4 months. Females were slightly more ruminative than males from age 12 , however, females did not report higher maladjustment until age 14. Furthermore, rumination and depression exhibited a bidirectional relationship across 4 months when measured in isolation, however when additional variables were assessed this relationship disappeared. In fact, anxiety, not rumination, predicted increases in maladjustment.

In combination, these studies show that although females report more maladjustment than males, this difference is small, supporting a gender similarities rather than a gender differences perspective. Second, rumination does not predict increases in either depressive or anxious symptoms during adolescence. Therefore, 
these findings challenge the applicability of RST to adolescent samples. It is concluded that rumination is a correlate, not a cause of depressive and anxious symptoms during adolescence. 


\section{Acknowledgements}

Many people have helped me along this dissertation journey, most of whom probably did so without even knowing it. There isn't nearly enough room to thank everyone that has influenced or encouraged me so apologies for those who are not mentioned by name.

First, thank you to my supervisor, Paul Jose for teaching me about the wonderful world of research, methods, and statistics. Throughout this process you have guided me through the inevitable problems and challenges involved in a thesis, while also encouraged me to grow as a researcher. Jim Vess also played a critical role by providing clinical expertise, as well as helping me break down what felt like, at times, an enormous workload into manageable chunks.

I could not have done any of this, of course, without the schools, parents, and adolescents who participated in this research, thank you. Various other people have been instrumental-Kim Grimshaw, who drew the cartoons for Study 1, and Joe Melser who conducted the boys' interviews in Study2. James Millington and Michael France were an enormous help with technical issues relating to the internet study. And the support staff upstairs, Ngarie Lavery, Nicola Panapa, and Jebi Jayapalan whose efficiency made this research so much easier to conduct.

My fellow postgraduates have been a constant source of stimulation, support, and friendship. In particular I would like to thank Chris Sibley and Andrew Robertson for their enthusiasm for life, and research. I would like to also thank the various Garry Lab members who have so warmly befriended me over the years, and other members of the Jose lab who have encouraged and supported me.

My oldest and dearest of friends, Rach and Ginny, your friendship extends well beyond the past 3 years, but you have both been invaluable throughout this process. You have kept me sane, and even entertained me while I ruminated (worried?) about my thesis. I would also like to make special mention of my family, who are always supportive of me, especially my parents, Graham and Helen Weir.

And of course, Matty G LP, my hubby, I don't even know how to describe how amazing you have been. Practically, you have read drafts, helped me with my graphs and figures, and made exceptional suggestions which no doubt have improved the final product of this thesis. More importantly however, you have always believed in me, and encouraged me to challenge myself, and my assumptions. I simply couldn't have done this without you. 


\section{Table of Contents}

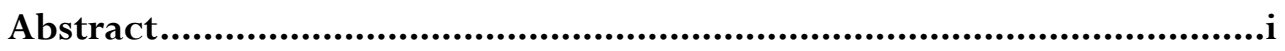

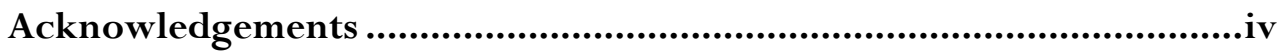

List of Tables ...................................................................................... viii

List of Figures.............................................................................. ix

Chapter 1: Introduction .................................................................. 1

Sex Differences in Depression ............................................ 2

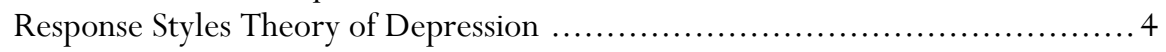

Empirical Support for Response Styles Theory in Adult Samples.................6 6

Response Styles Theory Applied to Children and Adolescents ................. 11

Overview of Studies .................................................. 20

Chapter 2: Concurrent Relationships among Stress, Rumination, Sense of Control, and Depressive Symptoms ................................................224

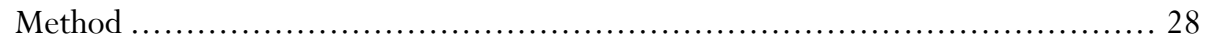

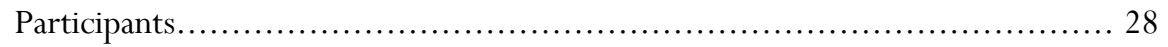

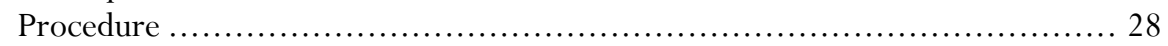

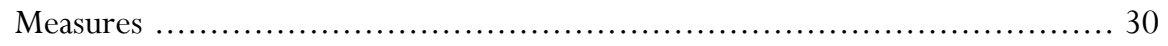

Results .............................................................. 32

Analytic Strategy ..................................................... 32

Developmental Trends of Stress, Sense of Control, Rumination, and Depressive

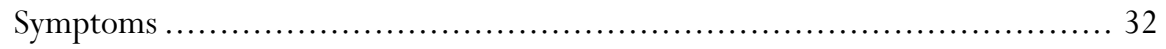

Relationships Among Stress, Rumination, Sense of Control, and Depressive

Symptoms. ....................................................... 35

Moderation Analyses ................................................. 35

Mediation Analyses ................................................. 37

Discussion .......................................................... 43

\section{Chapter 3: Parental and Peer Influences in Developing a Ruminative} Response Style During Preadolescence................................................ 49

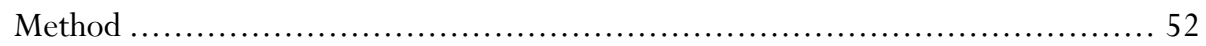

Participants and Procedure................................................. 52

Preadolescent Questionnaire ........................................... 53

Parental Questionnaire ............................................. 56

Results ............................................................... 57

Parental Encouragement of Rumination Based on Children's Age and Gender ... 57

Preadolescents Self Reported Levels of Rumination ........................ 58

The Relationship Between Parental Encouragement of Rumination and Self

Reported Rumination Among Preadolescents.............................. 59

Preadolescents Expectations of Rumination among their Peers ................. 61

The Relationship Between Self Reported Rumination and Preadolescents

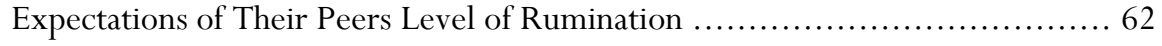

Discussion ........................................................... 62 
Chapter 4: The Development of a Scale to Measure Perceptions of False Self for Adolescents: Reliability, Validity, and Longitudinal Relationships with Maladjustment ......................................................6 69

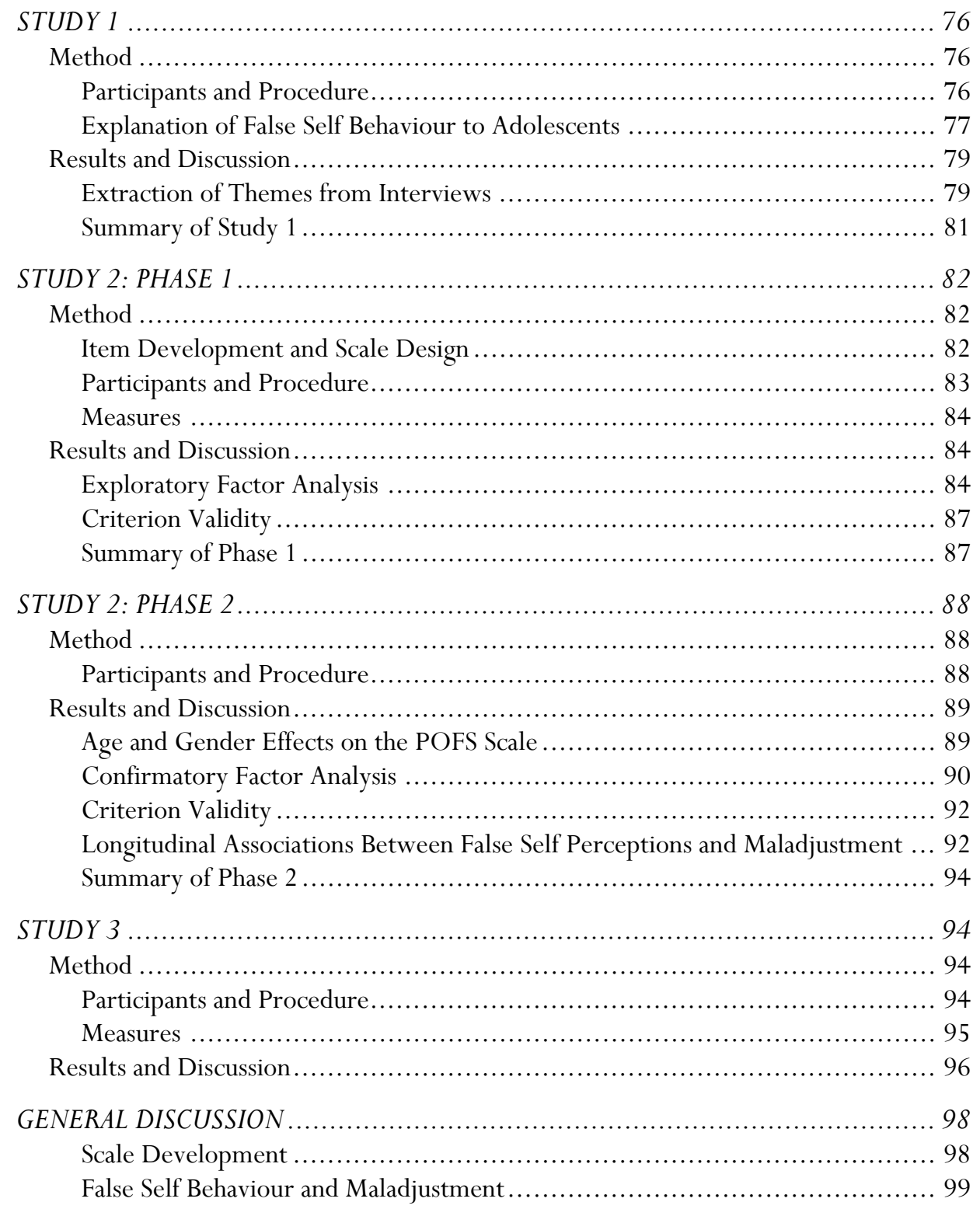

Chapter 5: The Relationships Among Stress, Rumination, Sense of Control, Perceptions of False Self, and Maladjustment Across 10 Weeks

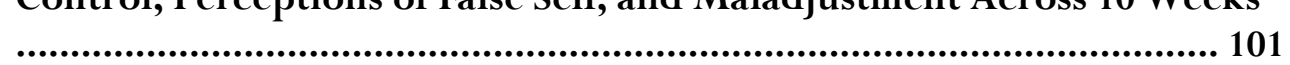

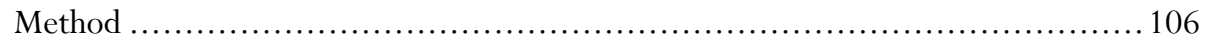

Participants and Procedure .................................................. 106

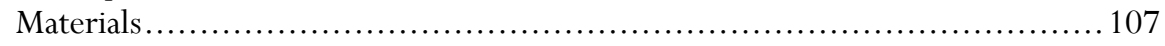

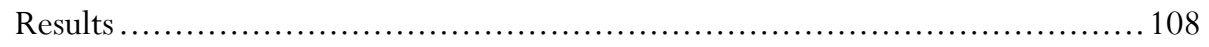

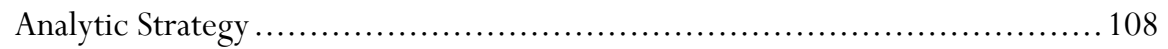

Developmental Trends of Stress, Sense of Control, Rumination, and

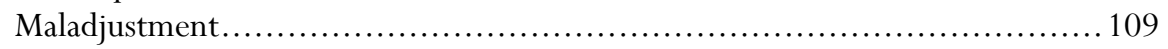

Relationships Among Stress, False Self, Sense of Control, Rumination, and

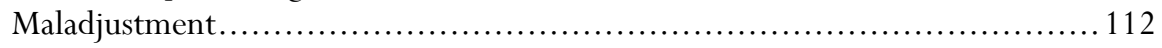


Mediational Model Replication........................................... 116

Directions of Effect Among Stress, Sense of Control, Rumination, Perceptions of

False Self, and Maladjustment Across 10 weeks............................. 118

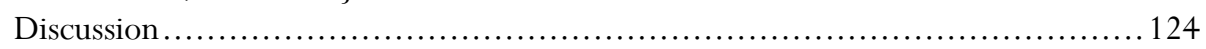

Chapter 6: The Relationships Among Stress, Rumination, Sense of Control, Perceptions of False Self, and Depressive and Anxious Symptoms......................................................................................... 128

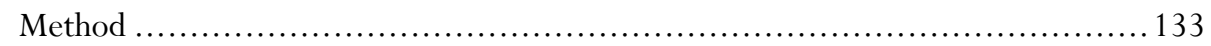

Participants and Procedures .............................................. 133

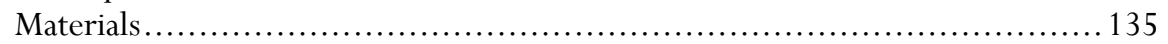

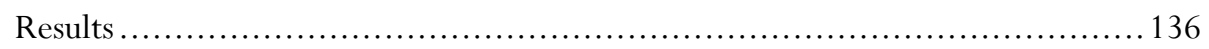

Analytic Strategy ................................................... 136

Developmental Trends of Stress, False self, Rumination, Sense of Control, and

Depressive and Anxious Symptoms. ...................................... 136

Relationships Among Stress, False Self, Sense of Control, Rumination, Anxious,

and Depressive Symptoms ............................................... 142

Construct Validity and the Direction of Effect among Risk Factors and

Maladjustment ............................................................. 144

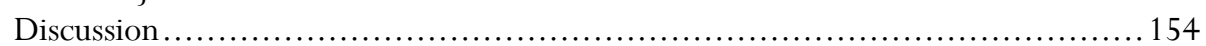

Chapter 7: General Discussion.................................................... 158

Sex Differences in Risk, Vulnerability, and Maladjustment During Adolescence 158 Response Styles Theory Applied to Adolescence: Does Rumination Prompt

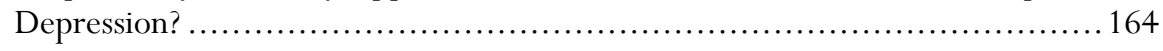

Applications to Clinical Depression .................................. 170

Conclusion .................................................... 170

References.............................................................................. 173

Appendix A: Global Stress Scale ................................................. 192

Appendix B: Overall Sense of Control Scale ....................................... 193

Appendix C: Ruminative Response Styles Questionnaire ................... 194

Appendix D: The Children's Depression Inventory ............................ 195

Appendix E: Stressful Scenarios presented to Preadolescents............. 197

Appendix F: Stressful Scenarios presented to Parents ......................... 206

Appendix G: Perception of False Self Scale ........................................ 215

Appendix H: The Revised Manifest Anxiety Scale for Children ......... 217

Appendix I: The Say What I Think Around Others Scale .................... 218

Appendix J: The Silencing the Self Scale ............................................ 219

Appendix K: Everyday Life Events Scale For Children........................ 220

Appendix L: Beck Anxiety Inventory ............................................. 222 


\section{List of Tables}

Table 2.1. Correlations among Stress, Rumination, Sense of Control, and

Depression

Table 2.2. Summary of Results of Rumination and Sense of Control Moderating the

Relationship Between Stress and Depression............................. 37

Table 3.1. Order of Scenarios Presented in Each of the Six Base Versions ......... 55

Table 3.2. Means and Standard Deviations for Male and Female Responses for Self

and Other Conditions................................................. 59

Table 3.3. Correlations Among Parental Encouragement of Rumination and Child

Self-Reported Rumination using Total Scores, Domain Scores, and RSQ

Scores ......................................................... 60

Table 4.1. Factor Loadings for the 1-Factor and 2-Factor Models of the POFS Scale

Table 4.2. POFS Means and Standard Deviations for Males and Females at Both

Time Points. 90

Table 4.3. Correlations Among Self Perception Scores and Depressive Symptoms.

Table 5.1. Within Subject Effects on Stress, Sense of Control, Rumination, and

Maladjustment

Table 5.2. Between-Subject Effects on Stress, False Self, Control, Rumination, and

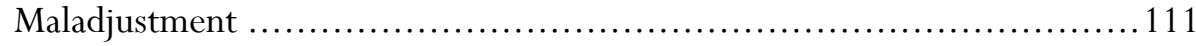

Table 5.3. Concurrent Correlations Among Stress, False Self Perceptions, Sense of

Control, Rumination, and Maladjustment.

Table 5.4. Longitudinal Relationships Among Stress, False Self Perceptions, Sense of

Control, Rumination, and Maladjustment.

Table 5.5. Results of Stress-Diathesis Moderation Analyses

Table 5.6. Results of Moderation Analyses Where Vulnerability Factors Are

Hypothesized to Exacerbate Maladjustment ..............................116

Table 6.1. Scale Reliabilities for Each Age Group at Both Baseline and 4 Months 135

Table 6.2. Means and Standard Deviations for Adolescents on all Measured

Variables.

Table 6.3. Concurrent Correlations Among Stress, False Self Perceptions, Sense of

Control, Rumination, Anxiety, and Depression.

Table 6.4. Longitudinal Relationships Among Stress, False Self Perceptions, Sense of

Control, Rumination, Anxiety, and Depression.

Table 6.5. Correlations Among Latent Variables for the Baseline and 4 Month

Measurement Models. 147 


\section{List of Figures}

Figure 2.1. Depressive symptoms reported by males and females aged 9-13 years

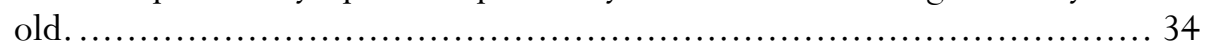

Figure 2.2. Rumination reported by males and females aged 9-13 years old....... 35

Figure 2.3. Rumination as a mediator between stress and depression ............. 38

Figure 2.4 Sense of control as a mediator between stress and depression.......... 39

Figure 2.5. Rumination and sense of control as simultaneous mediators between stress and depressive symptoms..................................... 41

Figure 2.6. Pruned structural mediational model where rumination and sense of control mediate the relationship between stress and depression............ 42

Figure 3.1. Parental encouragement of rumination for males and females aged 9-13 years. ............................................................ 57

Figure 4.1. Graphical depiction for adolescents of complete, partial, and no discrepancy between public and private self perceptions.................. 78

Figure 4.2. Confirmatory factor analysis of the POFS scale with 1-factor extracted 91

Figure 4.3. Cross lag path model between perception of false self and maladjustment across 10 weeks ..................................................... 93

Figure 5.1. Trends by age and gender on sense of control.....................111 112

Figure 5.2. Trends by age and gender on maladjustment ....................... 112

Figure 5.3. Mediation model replication....................................... 117

Figure 5.4. Modified mediation model ....................................... 117

Figure 5.5.Direction of effect between rumination and maladjustment across 10

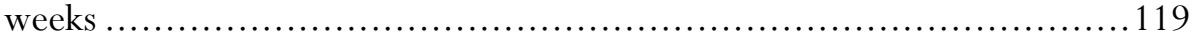

Figure 5.6. Pruned direction of effects model for the full sample................121

Figure 6.1. Reported rates of stress by males and females aged 11-16 yrs........140

Figure 6.2. Reported rates of depressive symptoms by males and females aged 11-16

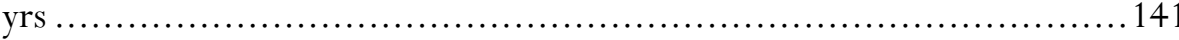

Figure 6.3. Reported rates of rumination by males and females aged 11-16 yrs...142

Figure 6.4. Measurement model for baseline variables.......................... 146

Figure 6.5.Direction of effect between rumination and depression across 4 months

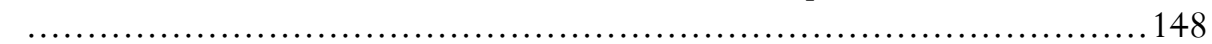

Figure 6.6. Pruned direction of effect model without anxious symptoms .........151

Figure 6.7. Direction of effect model with anxiety included for the total sample.153 


\section{Chapter 1: Introduction}

Adolescence is a time of considerable change and development. For most, adolescence is navigated successfully without experiencing any serious emotional problems. However, for a significant portion, emotional disorders such as depression can be debilitating. In a recent study of over 12,000 New Zealand Youth (9-13 years old), (2003) found that nearly 9\% of males and nearly $19 \%$ of females reported elevated levels of depressive symptoms. Given that depression is one of the most common forms of disability worldwide (World Health Organisation, 2007), studying the antecedents of depression during adolescence is critical to establish both preventative measures and effective treatment programs. Identifying the predictors of depression during adolescence is particularly important because depression during childhood and adolescence is often a precursor to adult depression (Harrington, Fudge, Rutter, Pickles, \& Hill, 1990; Kandel \& Davies, 1986).

Theoretical interest into childhood and adolescent depression has increased substantially over the last few decades. Various theoretical models explaining depression in adults have been extended downwards to explain depression in children and adolescents. Extending adult theoretical models of depression to adolescence is a promising endeavor because the phenomenology of depression is experienced somewhat similarly across the lifespan (Carlson \& Kashani, 1988;

Kovacs 1992). In this thesis, I test the applicability of the Response Styles Theory of depression (RST; Nolen-Hoeksema, 1987; 1990; 1991) in preadolescent and adolescent samples across a series of three studies. RST was originally developed to help explain one of the most intriguing and consistent findings relating to 
depression-depression is experienced up to twice as frequently among females (Weissman, Bland, Joyce, Newman, Wells, \& Wittcen, 1993). Two recent review articles (Hankin \& Abramson, 1999; Kuehner, 2003) have highlighted RST's potential to help explain childhood and adolescent depression, or more specifically to help explain why the gender difference in depression emerges during adolescence. In this chapter I will review the evidence for $\operatorname{sex}^{1}$ differences in depression, and then review evidence for RST in both adult and adolescent populations.

Prior to reviewing this literature however, it is important to define what is meant by adolescence. Adolescence is the period of physical, emotional, and cognitive transition between childhood and adulthood. Adolescence is also a time of increased autonomy and growing financial independence from parents or caregivers. The exact timing of the onset and length of these transitions differs across societies however, typically in Western societies, adolescence begins with the onset of puberty, which occurs at approximately age 13 for females and 14 years for males (Tanner, 1990). For the purposes of the studies presented in this thesis participants between the ages of 9-12 years will be considered preadolescents, and participants between 13-17 years old will be considered adolescents ${ }^{2}$.

Sex Differences in Depression

Adults. In one of the first reviews examining sex differences in depression, Weissman and Klerman (1977) examined treated cases of depression, completed

\footnotetext{
${ }^{1}$ Within this thesis the term sex and gender will be used interchangeably. Often gender and sex are used to specify the gender role characteristics and the biological features of individuals respectively (Unger \& Crawford, 1992). However because gender role characteristics are not directly measured in this thesis the distinction will not be used.

${ }^{2}$ Age was used to distinguish between preadolescence and adolescence because the stage of physical and cognitive development of participants was not measured.
} 
suicides, suicide attempts, general population surveys of depressed mood, and depression in bereaved adults. In this comprehensive review, data were gathered from numerous countries including New Zealand, USA, Wales, Denmark, India, Iraq, Taiwan, Thailand, and England. Across all countries, and measures of depressed mood, the incidence of depression was twice as frequent among females as males. The preponderance of depression among females has been supported by other authors (Kessler, McGonagle, Swartz, Blazer, \& Nelson, 1993; Kuehner, 2003; Nolen-Hoeksema, 1990; Weissman et al., 1993)

Children and Adolescents. An interesting developmental trend has been well documented in adolescent samples- females begin reporting higher rates of depression from the beginning of adolescence (Allgood-Merten, Lewinsohn, \& Hops, 1990; Hankin, Abramson, Moffitt, Silva, McGee, \& Angell, 1998; Schwartz \& Koenig, 1996; Twenge \& Nolen-Hoeksema, 2002). Hankin et al. tracked the development of sex differences in depression longitudinally-every 2 years, across a period of 10 years-among 653 eleven to twenty one year olds. Results indicated that 11 year old males were more depressed than females; however 13 year old males reported similar levels of depressed mood to females. At age 15 however, females were significantly more depressed than males. Hankin et al. concluded that mid-adolescence is characterized by a period of increased risk of depression among females. In another example, Twenge and Nolen-Hoeksema conducted a metaanalysis including 310 studies involving 62,424 adolescents aged 8-16. Twenge and Nolen-Hoeksema found that prior to adolescence $(<13$ years), males and females reported similar rates of depressive symptoms. However from age 13, females reported significantly more depressive symptoms than males. In combination, these 
studies show that males are either more depressed, or report similar levels of depression to females during preadolescence, but from the beginning of adolescence females' start to report significantly more depressed mood than males. Then, throughout the lifespan, subsequent to adolescence, females continue to report significantly more depressed mood than males (Nolen-Hoeksema, 1990) ${ }^{3}$. Response Styles Theory of Depression

Numerous theories have been developed to explain the general occurrence of depression (for reviews see Nolen-Hoeksema 1987; 1990). However, very few theories have been able to account for the gender difference in depression described above. In contrast, RST was developed specifically to help explain the higher prevalence of depression among females. RST (Nolen-Hoeksema, 1987; 1990; 1991) asserts that the maintenance and exacerbation of depressed mood can be explained, in part, by the use of ruminative coping; females are thought to experience more depressed mood than males because they ruminate more than males. A ruminative response is defined as "repetitively focusing on the fact that one is depressed; on one's symptoms of depression; and on the causes, meanings, and consequences of depressed symptoms" (Nolen-Hoeksema, 1991, p. 569) ${ }^{4}$. For example, when feeling distressed a ruminator thinks over and over about what caused them to feel upset and all the negative outcomes which may result from their feelings, instead of taking active steps to relieve their mood. In this sense,

\footnotetext{
${ }^{3}$ There are two adult groups where levels of depression do not consistently differ between males and females: university and elderly samples (Nolen-Hoeksema, 1990).

${ }^{4}$ Rumination as examined in this thesis differs to stress-reactive rumination (Robinson \& Alloy, 2003). Stress-reactive rumination is not examined in this thesis, and all references to rumination relate to emotion-focused rumination as defined by RST. For a review on stress reactive rumination, see Spasojevic, Alloy, Abramson, Maccoon, \& Robinson (2004).
} 
rumination is characterised as a self-focused passive style of coping where no active steps are taken to try and relieve one's distress.

Rumination is characterised as a coping style because most ruminators believe they are finding solutions to their problems through rumination (Lyubomirsky \& Nolen-Hoeksema, 1993). The unfortunate paradox, however, is that rumination enhances the effects of negative thinking, interferes with problem solving, instrumental behaviors, and concentration, and reduces an individual's willingness to engage in enjoyable activities which could alleviate their mood (Lyubomirsky \& Nolen-Hoeksema, 1993; 1995). A variety of other researchers have asserted that self-focused attention can increase depressed mood (Ingram, 1990; Pyszczynski \& Greenberg, 1987; Teasdale, 1983). However RST describes a specific type of self-focus, which has been found to have a larger impact on negative mood than non-ruminative, more general self-focused attention (Mor \& Winquist, 2002)

RST originally asserted that males experience less depression than females because males tend to distract themselves in the face of distress (Nolen-Hoeksema 1987; 1990; 1991). Thus, according to RST, males tend to be more active in their responses to distress than females. However, this latter assertion has not been consistently supported by empirical findings (e.g. Broderick, 1998; Butler \& NolenHoeksema, 1994; Just \& Alloy, 1997; Nolen-Hoeksema, Parker \& Larson, 1994; Strauss, Muday, McNall, \& Wong, 1997; Ziegert \& Kistner, 2002). In recent times, the role of distraction in RST has been minimized (Nolen-Hoeksema, Wisco, \& Lyubomirsky, 2007). In light of this reconceptualisation, the current thesis only examines the predictions of RST associated with rumination, not distraction. 
Empirical Support for Response Styles Theory in Adult Samples

The two major tenets of RST are that females ruminate more than males, and that rumination prompts and/or maintains depressed mood. Research testing these two major tenets will be reviewed in turn.

Sex differences in rumination. The sex difference in rumination among adults has typically been assessed by measuring males and females on self-report surveys. The most commonly used questionnaire is the Response Styles Questionnaire (RSQ; Nolen-Hoeksema et al., 1994), however the Cognitive Emotion Regulation Questionnaire (Garnefski, Kraaij, \& Spinhoven, 2001) and the Rumination on Sadness Scale (Conway, Csank, Holm, \& Blake, 2000) have also been used to assess rumination. On such scales, females consistently report higher endorsement of ruminative coping than males (Butler \& Nolen-Hoeksema, 1994; Conway, Mendelson, Giannopoulos, Csank, \& Holm, 2004; Garnefski, Teerds, Kraaij, Legerstee, \& van den Kommer, 2004; Nolan, Roberts, \& Gotlib, 1998; NolenHoeksema, Larson, \& Grayson, 1999; Nolen-Hoeksema et al., 1994). One exception to this general finding is in university samples where gender differences in rumination are not consistently found (Lyubomirsky \& Nolen-Hoeksema, 1995; Morrison \& O’Connor, 2005; Nolen-Hoeksema \& Morrow, 1991; Weir, Jose, \& Pilcher, 2006). Recall that gender differences in depression are not consistently found in university samples either; therefore, it is possible that females with good mental health are over-represented in university samples (Nolen-Hoeksema, 1990).

Given that females generally tend to ruminate more than males, what factors may prompt increased rumination among females? Research examining this question has found that females tend to report higher levels of a variety of variables 
that predict rumination. For example, Nolen-Hoeksema et al. (1999) measured levels of stress, mastery, rumination, and depressive symptoms among 1132 adults from the general population across 1 year. Rumination at 1 year was predicted by higher baseline levels of stress and depressive symptoms, and lower levels of mastery. In this study, women scored higher than males on stress and depressive symptoms and lower on mastery, hence, Nolen-Hoeksema et al. suggested that females may ruminate more than males because they are more stressed and depressed, and also because they feel less control over their lives. Concurrently, rumination has also been predicted by neuroticism (Nolan et al., 1998; Roberts, Gilboa, \& Gotlib, 1998), which also tends to be higher among females (Nolan et al.). Nolen-Hoeksema and Jackson (2001) have also suggested that females may ruminate more than males because females believe emotions are more uncontrollable, and may therefore rely on passive coping strategies more than males. In essence, Nolen-Hoeksema and colleagues have argued that rumination is more common among females because females experience more stress, feel less control over their lives, and have more neurotic tendencies than males. Each of these factors is thought to give women material to ruminate about, which in turn is thought to contribute to their feelings of distress.

The relationship between rumination and maladjustment. In one of the first empirical tests of RST, Morrow and Nolen-Hoeksema (1990) induced sadness in participants who were then assigned to either a ruminative task or a distractive task. In the ruminative task, participants read statements which related to their feelings ("I often wonder why I feel the way I do"), and in the distractive task participants read statements about external events (“Canada's biggest industry is lumber"). Each task was 
also broken down further as either active or passive. In the active condition, participants were required to actively sort giant cards, and in the passive condition participants simply read the statements on the cards. Results indicated that the most reduction in negative mood was seen in the active-distractive condition, followed by the passive-distractive, active-ruminative, and then the passive-ruminative condition. Importantly, the mood of subjects in the active-distractive condition returned to pre-induction levels, however the passive-ruminative group's level of sadness remained stable. These results showed that rumination maintained maladjustment in participants with induced negative mood.

In an extension of this study, Nolen-Hoeksema and Morrow (1993) compared how ruminative and distractive responses influenced the moods of depressed and non-depressed individuals. Nolen-Hoeksema and Morrow showed that rumination maintained depressed mood only in currently depressed individuals. These findings have been replicated by a number of other researchers using experimental designs (Blagden \& Craske, 1996; Katz \& Bertelson, 1993). Furthermore, such results have been replicated using longitudinal survey-based designs (Nolan et al., 1998). Specifically, Nolan et al. measured rumination and depression across a 10 week interval, and found that the highest rate of depression at follow up were among participants who: (a) ruminated about their problems at baseline, and (b) exhibited elevated initial levels of depression. In short, Nolan et al. found that rumination exacerbated the relationship between initial and later depression.

Many additional survey-based studies have provided support for RST. In a now classic study, Nolen-Hoeksema and Morrow (1991) measured response styles 
in undergraduate students 2 weeks before the 1989 Loma Prieta Earthquake, as well as 10 days and 7 weeks after the earthquake. Nolen-Hoeksema and Morrow were interested in whether an individual's response style would predict their reactions to the earthquake across time. In support of RST, after controlling for baseline levels of depressed mood, initial levels of rumination predicted levels of depression both 10 days and 7 weeks after the earthquake. Nolan et al. (1998) have also shown that rumination can predict increases in depressive symptoms over and above what initial baseline levels of depression can predict across 7-10 weeks. Rumination also appears to predict changes in depressed mood across much longer periods of time. Nolen-Hoeksema et al. (1994) tested rumination’s ability to predict depression across 6 months in a sample of bereaved individuals. Nolen-Hoeksema et al. found that rumination significantly predicted levels of depression 6 months after the loss of a loved one, even while initial levels of depressed mood were controlled. However, rumination did not predict depressed mood across 1 year in a sample of bereaved males who had lost their partner to AIDS (Nolen-Hoeksema, McBride, \& Larson, 1997). Nolen-Hoeksema et al. (1997) suggested that this unanticipated result was the function of small sample size and increased additional stressors experienced by participants in this latter study.

Extending upon RST, Just and Alloy (1997) tested whether or not rumination could predict the onset of depressive episodes in a sample of initially non-depressed participants who were cognitively at risk of developing depression because of their attributional style. Findings supported Just and Alloy's predictions: rumination predicted the onset of depressive episodes across 18 months. NolenHoeksema (2000) also tested whether or not rumination could predict new episodes 
of depression. Nolen-Hoeksema found that rumination could predict the onset of new episodes of depression across 1 year. In combination, these results suggest that rumination can both maintain distress across time and predict new episodes of depression in previously non-depressed individuals.

RST has also recently been extended to help explain the exacerbation of anxiety. In particular, Nolen-Hoeksema (2000) assessed whether rumination could predict anxious mood across 1 year. Nolen-Hoeksema found that even after controlling for initial levels of anxiety, rumination predicted changes in anxious symptoms across time. The relationship between rumination and anxiety has also been supported by Flett, Madorsky, Hewitt, and Heisel (2002). Therefore, the effects of rumination may extend beyond depression to anxiety.

For rumination to be considered a vulnerability factor to depression, rumination must remain stable across long periods of time, and remain stable even in the absence of current depressive symptoms (Price \& Lento, 2001; Roberts et al., 1998). Typically, research has assessed the stability of rumination by examining the test-retest reliability of rumination across time. Using this criterion, rumination has been found to be stable across 2 weeks, $r=.87$ (Weir et al., 2006), 4 months, $r$ $=.79$ (Weir et al.), 6 months, $r=.80$ (Nolen-Hoeksema et al., 1994), and 1 year, $r=.67$ (Nolen-Hoeksema et al., 1999). In addition, Nolen-Hoeksema, Stice et al. (2007) found that among adolescent females rumination remained relatively stable $(r \mathrm{~s}=.32$ to .44$)$ even when measured at yearly intervals across a 4 year period. Therefore, rumination appears to be a trait-like vulnerability factor for depressed and anxious mood in adults. 
In summary, RST has received strong empirical support in adult samples in that rumination has been found to maintain depressed mood, predict changes in depression across time, and also predict the onset of new depressive episodes. However, it is not well understood if rumination influences the wellbeing of children and adolescents. Because the gender difference in depression emerges during adolescence, RST may be able to account, in part, for the onset of this gender difference and for depression in adolescence more generally. Response Styles Theory Applied to Children and Adolescents

In two reviews (Nolen-Hoeksema, 1994; Nolen-Hoeksema and Girgus, 1994), RST was extended downwards in age to explain the higher prevalence of depression among female adolescents. These authors argue that depression is higher among females during adolescence because females experience higher levels of risk factors during adolescence, and females enter adolescence with more ruminative response styles than males. Specifically, it is hypothesized that females are at a higher risk of depression because they face more biological and social stressors than males during adolescence, and when these stressors interact with ruminative tendencies, females become more depressed than males. Therefore RST predicts that depression is more prevalent among adolescent females because adolescence is more stressful for females, and females exhibit greater vulnerability for depression than males during this period. RST asserts that the causal mechanisms which prompt depression are the same for males and females, but because females experience greater risk and vulnerability to depression, they develop depression more frequently than males (Nolen-Hoeksema, 1994). 
In essence, RST as applied to adolescence is a diathesis-stress model of depression, where the combination of increased risk (stress) and vulnerability (rumination) maintains and exacerbates negative mood (Nolen-Hoeksema, 1994; Nolen-Hoeksema \& Girgus, 1994). In diathesis-stress models, risk and vulnerability are characterized as distinct constructs (Compas \& Phares, 1991; Ingram \& Price, 2001). Risk factors are those associated with an elevated probability of developing depression, whereas vulnerability factors are conceptualized as the mechanisms which prompt disorders (Ingram \& Price). For example, gender, stress, and prior depressive symptoms are well documented risk factors for developing depression (Garber \& Flynn, 2001). Females, adolescents under stress, and adolescents who have experienced depressive symptoms in the past have an increased probability of developing depressive symptoms. However, not gender, stress, or prior depressive symptoms can explain why depression occurs on their own.

In contrast, vulnerability factors are considered causal mechanisms for the development of disorders (Ingram \& Price, 2001). Vulnerability factors are either biologically or environmentally based trait-like characteristics of an individual that exist across time regardless of an individual's current state of psychopathology (Price \& Lento, 2001). On their own, vulnerability factors are not thought to prompt maladjustment, however when vulnerability factors are triggered by stressful events, maladjustment is expected to occur. To date, diathesis-stress models with cognitive vulnerability factors such as dysfunctional attributional style have gained substantial support (for a review see Scher, Ingram, \& Segal, 2005), however, less research has tested whether rumination interacts with stress to prompt depression in the way RST would predict. Rumination has initial support as 
a vulnerability factor for depression in that it appears to be a stable trait-like variable in adolescence, as has been seen in adults (Driscoll, 2004; Nolen-Hoeksema, Stice, Wade, \& Bohon, 2007).

Next, the major tenets of RST as applied to adolescents will be reviewed. Specifically, research examining whether preadolescent and adolescent females are more ruminative will be examined, followed by the research that examines whether stress and rumination maintain and exacerbate depression during adolescence.

Sex differences in rumination. Although the developmental trajectory of depression is well understood (Allgood-Merten et al., 1990; Hankin et al., 1998; Twenge \& Nolen-Hoeksema, 2002), there is little research on the developmental trajectory of rumination. If rumination is to be considered a predisposing vulnerability factor for the gender difference in depression, rumination needs to be more prevalent among females prior to, or occur simultaneously with, the development of the gender difference in depression. To examine this question, Broderick (1998) tested whether females reported ruminating more frequently than males in preadolescents aged 9-12 years. In this study, preadolescents indicated how much they would ruminate about family, peer, and academic stressors. Across all three domains, females reported they would ruminate in response to stress significantly more than males. Broderick concluded that this result showed rumination was a significant vulnerability factor for the development of the gender difference in depression. However, Broderick did not adequately test whether females were more depressed in this sample; depressed mood was assessed using a 1-item measure of sadness. Thus, although this study demonstrated that female preadolescents self-report rumination at higher levels than males, it did not 
demonstrate that rumination precedes the gender difference in depression or demonstrate that rumination is related to depression in preadolescence.

In a series of later studies, Broderick and Korteland (2002) found that $4^{\text {th }}$ grade females ruminate more than $4^{\text {th }}$ grade males, yet females did not report more depressed mood than males until the $5^{\text {th }}$ grade. These results suggest that rumination is endorsed more frequently in females prior to the development of gender differences in depression, supporting the application of RST to preadolescence. In addition, Broderick and Korteland found that males and females were expected to behave in gender-typed stereotypical fashion by other adolescents. Specifically, in response to vignettes depicting male and female protagonists in stressful situations, adolescents expected female protagonists to ruminate more, whereas male protagonists were expected to distract more. Other researchers have found that the gender difference in rumination either precedes that of depression (Jose \& Brown, 2007; Ziegert \& Kistner, 2002) or occurs simultaneously (Muris, Roelofs, Meesters, \& Boomsma, 2004; Schwartz and Koenig, 1996). Interestingly, in samples where there has been no gender difference in rumination, there has also been no significant gender difference in depressive symptoms (Abela, Brozina, \& Haigh, 2002; Broderick \& Korteland, 2004). These results provide preliminary evidence in support of the RST's application to depression in adolescence. However, most studies to date have collapsed analyses across age, so subtle developmental changes in the prevalence rates of rumination remain unclear.

One of the only studies designed specifically to investigate the developmental trends of rumination was conducted by Jose and Brown (2007). Jose and Brown measured rumination using the RSQ in 1,218 10-17 year olds, and 
found that 12 year old females reported significantly more rumination than males; this gender difference in rumination persisted throughout the remaining age groups. Thus, the developmental trend of rumination differed for males and females. Females dramatically increased their levels of rumination from age 13 years, whereas males ruminated at a relatively consistent rate in subsequent year groups suggesting that it is not that males reduce their levels of rumination across adolescence, but rather females increase their levels of rumination. Furthermore, females did not report higher levels of depressive symptoms until age 13, showing that the gender difference in rumination precedes the development of the gender difference in depressive symptoms. This is one of the few studies investigating the developmental trends of rumination, but because it examined the trends using concurrent data, its findings require replication, preferably with longitudinal samples.

The relationship between rumination and maladjustment. Various studies have shown that rumination in child and adolescent samples are associated with increased maladjustment (Garnefski, Boon, \& Kraaij, 2003; Muris, Roelofs et al., 2004; Ziegert \& Kistner, 2002). These findings provide preliminary support for the basic tenets of RST being extended downward to children and adolescents. Additional studies have tested the explicit extension of RST that stress interacts with rumination to predict depressive symptoms. Using concurrent data, Kraaij et al. (2003) found a significant stress by rumination interaction on depressive symptoms, among adolescents ${ }^{5}$.

\footnotetext{
${ }^{5}$ However, whether the variables were centred in the moderation model were not specifically stated, nor was the interaction term plotted, therefore this result requires replication.
} 
The first longitudinal study which examined RST's predictions in an adolescent sample was conducted by Schwartz and Koenig (1996). Schwartz and Koenig examined whether rumination, negative life events, and negative attributional styles could predict depressive symptoms across 6 weeks in a sample of 14-18 year olds. After controlling for initial levels of depression, neither attributional style nor negative life events predicted depression, however, initial levels of rumination predicted depression 6 weeks later. None of the interactions between negative life events and cognitive styles however, predicted later depression. Similarly, Abela et al. (2002) found that rumination significantly predicted depression scores across 6 weeks, while initial levels of depression were controlled. Abela et al. did not measure stress, and therefore the extended RST could not be tested in this sample. These results provided preliminary support for RST in that rumination predicted changes in depressive symptoms in adolescents; however stress and rumination did not interact in Schwartz and Koenig's study to predict depression longitudinally.

In contrast, Driscoll (2004) found that stress did interact with rumination to predict depressive symptoms across 9 months in older adolescents $\left(6^{\text {th }}-7^{\text {th }}\right.$ grade) . However, the direction of the interaction was not in the predicted fashionrumination was most predictive of depression in adolescents not currently experiencing stress. Methodological limitations of this study suggest these results require verification. Specifically, the variables did not appear to be centered before creating the interaction terms, which can intensify problems with multicollinearity (Holmbeck, 1997; Howell, 2002). Thus, Kraaij, et al. (2003) was the only study which specifically supported the predictions of Nolen-Hoeksema (1994) and Nolen- 
Hoeksema and Girgus (1994). In this thesis, the specific predictions of RST as applied to adolescents will be examined further.

Recall that RST has recently been extended in the adult literature to help explain the exacerbation of anxiety (Nolen-Hoeksema, 2000). Similarly, in adolescent samples, higher levels of rumination are associated with higher levels of anxiety (Driscoll, 2004; Garnefski et al., 2001; Muris, Roelofs et al., 2004; Schwartz \& Koenig, 1996). Longitudinally, Schwartz and Koenig, and Driscoll tested whether a ruminative response style could predict increases in anxious symptoms across 6 weeks and 9 months respectively. For both studies, rumination predicted increases in later anxious symptoms, even after initial levels of anxiety were taken into account. In combination, these preliminary studies suggest that the effects of rumination may extend beyond depressive symptoms, to anxious symptoms in adolescent samples.

Although a growing body of literature supports the basic tenets of RST in both adult and adolescent samples, there are at least three major limitations of existing literature relating to the association between rumination and depression. First, it is not clear how the relationship between rumination and depression is affected by other associated variables, or how rumination compares with other cognitive vulnerability factors in predicting depression, such as perceived control and perceptions of false self. A decreased sense of control over one's life has long been associated with a range of psychological ailments, including childhood depression (Donnelly, 1999; Muris, Meesters, Schouten, \& Hoge, 2004). Similarly, perceiving oneself as lacking authenticity (false self perception) is associated with depressed mood during adolescence (Harter, Marold, Whitesell, Cobbs, 1996). 
However rumination is rarely compared with other vulnerability factors of depression. In one of the only published studies assessing additional vulnerability factors to rumination in adolescence, Abela et al. (2002) found that hopelessness and rumination were correlated, and partial support was found for the hypothesis that hopelessness mediated the relationship between rumination and depression.

False self perceptions were compared with rumination in this thesis because false self perceptions are conceptually similar to the self-silencing hypothesis (Jack, 1991). The self-silencing hypothesis is another prominent theory of why females are more depressed than males. Specifically, the self-silencing hypothesis states that females tend to silence their needs and feelings within close intimate relationships more than males, which results in elevated levels of depression for females. Selfsilencing is very similar to the definition of false self behaviour asserted by Harter (2002) as 'lack of voice'. However, because very few adolescents are likely to be in close intimate relationships, self-silencing may not be appropriate to account for the gender difference in adolescent depression. For this reason, false self perception and not self-silencing was assessed in this thesis.

Furthermore, by comparing the effect of rumination, sense of control, and perceptions of false self on depression, it is possible to also examine the relationships between rumination and other cognitive vulnerabilities of depression. Very few studies have done this, however, it is likely that rumination, sense of control, and perception of false self are interrelated. Rumination (by definition), occurs within a context of distress, and the reasons for this initial distress may relate to sense of control of perceptions of false self. For example, an adolescent may be ruminating about their false self behaviour, or their perceived lack of control over 
their lives, which in turn increases rumination and distress. Alternatively, ruminating about ones distress may result in additional or intensified feelings of lack of control, or false self perceptions, which in turn may increase distress. This thesis will explore the interrelationships among these cognitive vulnerabilities of depression, among preadolescent and adolescent samples. In summary, the first overarching goal of this thesis is to compare rumination with other vulnerability factors of maladjustment. Specifically, in this thesis rumination's predictive ability is compared with sense of control as well as perceptions of false self.

The second major limitation of existing research testing the predictions of RST is that most studies only test one direction of effect (causality) among rumination and depression-specifically most studies test whether rumination increases depression across time. This is likely to be because RST asserts that rumination can lead to depression, thus placing large emphasis on the effect of rumination on depression. However, multiple alternative directions of effect are possible (Arnow, Spangler, Klein, \& Burns, 2004). One possibility is that the relationship between rumination and depression is reciprocal. In other words, rumination and depression may prompt increases in each other simultaneously (Nolen-Hoeksema, Stice et al., 2007; Teasdale, 1983; Weir et al., 2006). In adolescent populations, Nolen-Hoeksema, Stice et al. are some of the few researchers who have tested alternative directions of effect; however their data only includes females. Nolen-Hoeksema, Stice et al.'s results suggest that rumination and depression effect each other reciprocally across a period of 4 years. This result is also consistent with research conducted with adult samples (Weir et al., 2006). Interestingly, in Nolen-Hoeksema, Stice et al.'s and Weir et al.'s studies the general 
trend of effect was stronger from depression to rumination than the reverse.

Therefore, another overarching goal of this thesis is to assess alternative directions of effect among rumination and depression.

The third limitation of existing literature is related to the construct validity of rumination. It is possible that the association commonly found among rumination and depression may be the result of construct overlap (Roberts et al., 1998). Furthermore, it is possible that the association between rumination and depression may be the result of spurious associations with additional variables (Arnow et al., 2004). Treynor, Gonzalez, and Nolen-Hoeksema (2003) tested the former issue using exploratory factor analysis and found support for the construct validity of rumination. However, additional research is needed to replicate the construct validity of rumination using more stringent tests. The latter issue, that the association between rumination and depression is spurious, is important to test because preliminary research suggests that the association between rumination and depression over time may be best accounted for by a common cause (also known as the spurious model) (Arnow et al.). Therefore, a further goal of this thesis is to test the construct validity of rumination and depression, and explore the interrelationships with correlated constructs (for example, anxiety) to test for spurious associations.

Overview of Studies

Across a series of three studies, RST is tested using adolescent and preadolescent samples. Study 1 is described in two parts, across Chapters 2 and 3. In Chapter 2, the developmental trends of rumination and depressive symptoms are assessed in preadolescents aged 9-13 years. If females are found to be more 
ruminative than males prior to the age at which females report more depressive symptoms than males, it will provide support for Nolen-Hoeksema's (1994) assertion that rumination is a vulnerability factor for depression with which females enter adolescence more frequently than males. In addition, Chapter 2 assesses the interrelationships among stress, rumination, sense of control, and depressive symptoms to explore whether risk and vulnerability interact to predict depressive symptoms, and also reports findings that allow a comparison of the ability of rumination and sense of control to predict depressive symptoms. These results will show how powerful rumination is compared to other established risk factors for predicting depression in adolescence.

Then in chapter 3, the same preadolescents responded to cartoon scenarios depicting males and females in stressful situations. It was tested whether preadolescents expect females to ruminate in the face of stress more than males. In addition, the parents of these preadolescents responded to the same cartoon scenarios as their children, but indicated whether they would encourage their children to ruminate; it was tested whether parents encourage female preadolescents to ruminate more than males. If parents encourage females to ruminate more than males, and peers expect female preadolescents to be more ruminative than male adolescents, support will be provided for Nolen-Hoeksema's (1991) assertion that societal expectations and pressures encourage greater use of rumination among females.

Study 2 is also described in two parts, across chapters 4 and 5 . Chapter 4 introduces an additional vulnerability factor, perceptions of false self, to be compared with rumination as a predictor of maladjustment. In chapter 4 the 
development of a scale measuring false self perceptions is described in a sample of 11-15 year olds. A new false self scale is developed because the format of existing scales is confusing to adolescents, and present measures have not been subjected to strict tests of psychometric properties. The new scale is developed using a multimethod approach beginning with interviews to develop scale items, followed by exploratory and confirmatory factor analysis to test the reliability and validity of the scale. And last, the newly developed scale is compared with existing measures of false self behaviour to predict maladjustment.

Then in chapter 5, the interrelationships among stress, false self, rumination, sense of control, and maladjustment are examined across 10 weeks using the same participants as are described in chapter 4 . The diathesis-stress models are tested and compared. In addition, the directions of effect among the measured variables are explored using structural equation modeling. If the direction of effect shows that rumination can predict depressive symptoms across time then the predictions of RST will be supported.

Chapter 6 describes study 3 where a large sample of 11-16 year olds were recruited to replicate and extend upon the findings presented in chapter 5. Adolescents were assessed on measures of stress, sense of control, rumination, perceptions of false self, and anxious and depressive symptoms across 4 months. Study 3 had three major goals. The first goal was to compare how stress, sense of control, false self, and rumination predict depressive and anxious symptoms. The second goal was to explore the construct validity of each measured variable. And the last goal was to test more thoroughly the direction of effect among these variables. The two latter goals of study 3 were tested using a 2-step latent variable 
path modeling approach (Anderson \& Gerbing, 1988). For RST to be supported, the construct validity of rumination needs to be verified, and rumination needs to predict depressive and anxious symptoms across time. Finally, in chapter 7, the major findings of this series of studies will be discussed in light of the theoretical implications for RST. 


\section{Chapter 2: Concurrent Relationships among Stress, Rumination, Sense of Control, and Depressive Symptoms}

In the current chapter I examine how stress, rumination, sense of control, and depressive symptoms are interrelated in a preadolescent sample. There are five objectives of the current study. First, I test whether the gender difference in rumination is evident in preadolescence. That is, do females ruminate more than males prior to the onset of the gender difference in depression? If rumination is more prevalent among females during preadolescence, then rumination may act as a vulnerability factor that influences the gender difference in depression, as NolenHoeksema (1994) and Nolen-Hoeksema and Girgus (1994) suggest. However if the gender difference in depression precedes the development of the gender difference in rumination, it would suggest that higher rumination among females may not account for the gender difference in depression. It was predicted, based on previous research (Jose \& Brown, 2007; Twenge \& Nolen-Hoeksema, 2002; Ziegert \& Kistner, 2002), that the gender difference in rumination would be evident in this preadolescent sample, and that there would not be a gender difference in depression.

The second goal of the current study was to examine whether rumination influences the relationship between stress and depression. Examining the ways in which rumination influences the relationship between stress and depression can empirically test the diathesis-stress model proposed by Nolen-Hoeksema (1994) and Nolen-Hoeksema and Girgus (1994). Empirically, a stress-diathesis model of depression can be tested using moderation analyses in which rumination is conceptualized as a moderator of the relationship between stress and depression (Cole \& Turner, 1993). Recall that in a diathesis-stress model, when under stress 
(risk factor), an adolescent with a ruminative tendency (vulnerability factor present) will experience greater distress than an adolescent without a ruminative tendency (vulnerability factor absent). To date, a number of studies have failed to support a diathesis-stress model (Sarin, Abela \& Auerbach, 2005; Schwartz \& Koenig, 1996). On the other hand, other studies have supported a stress-diathesis model, for example, Kraaij et al. (2003) found that rumination moderated the relationship between stress and depression in an adolescent sample. In the current study, I tested the diathesis-stress model of depression using moderation analyses (Baron \& Kenny, 1986; Holmbeck; Howell). Based on Kraaij et al. and the theoretical predictions of Nolen-Hoeksema and Girgus, I predicted that rumination would show a stronger relationship with depressive symptoms in preadolescents who were under stress. In short, I predicted that rumination would moderate the relationship between stress and depressive symptoms in the current sample.

Another way rumination could influence the relationship between stress and depressive symptoms is to act as a mediator, and in this capacity it can help explain how a stressed individual comes to experience depressive symptoms ${ }^{6}$. If rumination is found to be a mediator between stress and depression, it would suggest that stress prompts an individual to ruminate about his/her problems, which in turn increases an individual's feeling of distress. Rumination was conceptualized as a potential mediator in this study because rumination has been found to mediate the relationship between stress and depression in adult populations (NolenHoeksema et al., 1999), however, it is unknown if rumination acts as a mediator between stress and depression during preadolescence. It was predicted that

\footnotetext{
${ }^{6}$ Conceptually and empirically, it is possible for a variable to be both a mediator and moderator of a relationship (Baron \& Kenny, 1986).
} 
rumination would mediate the relationship between stress and depression, and hence be a mechanism which helps translate stress into depressive symptoms.

The third goal of this study was to compare rumination with sense of control, which is another well known vulnerability factor of depressive symptoms. As described earlier, a decreased sense of control over one's life has long been associated with a range of psychological ailments, including childhood depression (Donnelly, 1999; Muris, Meesters et al., 2004). Rumination and sense of control were compared as both mediators and moderators of the relationship between stress and depression. Traditionally, perceived control has been conceptualized as a moderator of the relationship between stressful life events and depression, however more recently, sense of control has also been conceptualized as a mediator of this relationship in child and adolescent populations (Cole \& Turner, 1993; Gibb \& Alloy, 2006). Sense of control is therefore considered to be a possible causal mechanism that transforms stress into depressed mood. It is not well understood how rumination compares with other known risk factors of depression in preadolescent populations such as perceived control. Therefore, a goal of the current study was to compare the relative strengths of rumination and perceived control as both mediators and moderators of the relationship between stress and depressive symptoms. There were no predictions made as to which vulnerability factor would better predict depressive symptoms.

The fourth goal of the current study was to explore the association between perceived control and rumination. Within adult samples, lower levels of perceived control (mastery) are associated with higher levels of rumination (Nolen-Hoeksema et al., 1999; Wanke \& Schmid, 1996). Within adolescent populations, Abela et al. 
(2002) have suggested that a lack of perceived control over one's life may mediate the relationship between rumination and depression. For example, rumination may prompt an adolescent to feel less control over his or her life, which in turn, may increase the adolescent's depressed mood. However, Abela et al. found mixed results for perceived control as a mediator between rumination and depression, and therefore this possibility needs to be explored further. I predicted that the relationship between rumination and depressive symptoms would be mediated by perceived control.

The final goal of the current study was to examine the impact that age and gender had on the interrelationships among stress, rumination, sense of control, and depressive symptoms. Various studies have found that females report lower perceived control over their lives than males (Nolen-Hoeksema et al., 1999) and ruminate more than males during adolescence (Jose \& Brown, 2007). However, theoretically, the effects of stress and rumination are not thought to differ between males and females in either adulthood or adolescence (Nolen-Hoeksema, 1990). Thus, I did not expect the interrelationships to be moderated by gender. I also examined if the relationships among stress, rumination, perceived control, and depressed mood were different for younger (ages 9-10 years) and older preadolescents (11-13 years old). Previous research has found that the effect of selffocused attention on maladjustment may be stronger for adults than adolescents (Mor \& Winquist, 2002), however because of the narrow age range in this study, differences between younger and older preadolescents were not expected. 


\section{Method}

Participants

Preadolescents. Initially, 320 preadolescents participated in this study;

however 10 of these participants completed less than $95 \%$ of the survey and were deleted from the sample (Tabachnick \& Fidell, 2001) ${ }^{7}$. The final sample included 310 preadolescents (138 males and 172 females) obtained from 12 schools throughout the Central New Zealand region. Regarding the socio-economic status of the schools, two schools were decile 3 , one school was decile 4, one school was decile 5 , two schools were decile 7 , two schools were decile 8 , and a further four schools were decile $10^{8}$. The sample was composed of 72 nine-year-olds (30 males, 42 females), 86 ten-year-olds (38 males, 48 females), 67 eleven-year-olds (26 males, 41 females), 55 twelve-year-olds (30 males, 25 females), and 30 thirteenyear-olds (14 males, 16 females). The majority of children (63.2\%) were European

New Zealanders, $12.3 \%$ were Maori, $4.2 \%$ were Pacific Islanders, $3.2 \%$ were Asian, $17.7 \%$ classified their ethnicity as "Other", and one participant did not provide information about ethnicity. These frequencies are representative of New Zealand's ethnic distribution.

Procedure

I sent introductory letters to the principals of 41 schools in the Central North Island Region. A follow up phone call was made to the principal to obtain an expression of interest in the study. If a school wished to participate, they designated a contact person for the project who provided class lists for each child eligible to

\footnotetext{
${ }^{7}$ Deleting these participants from the sample was not expected to affect the results in any way because the deleted participants comprised less than $5 \%$ of the initial sample (Tabachnick \& Fidell, 2001). ${ }^{8}$ School deciles are calculated based upon the household income, occupation of parents, amount of household crowding, educational qualifications, and whether or not income support is being received. Deciles can range between 1 (low SES) and 10 (high SES)
} 
participate in the project ${ }^{9}$. Each school was provided with enough recruitment packages for every child eligible to participate in the project. In total 1161 parental packages were provided to schools ${ }^{10}$. This recruitment package included a parental information sheet, a parental questionnaire, and a parental consent form for their child's participation. These packages were taken home to parents by children.

Within the package was a postage-paid self addressed envelope so parents could return the completed parental questionnaires in a quick, confidential manner. Signed parental consent forms were returned to the school prior to data collection. In all cases, data were collected at schools, during class time. These data were collected in groups of children ranging in size between 10-30 children. Assent from the children to participate was obtained prior to data collection. Data collection was voluntary and participants could withdraw at anytime. The children were told that the research investigated how adolescents saw themselves, what sorts of events happened in their lives, and how they felt. The research was conducted in a quiet room designated by the school, e.g. school library or empty classroom. In most cases the teacher was not present. When the teacher was present, it was because non-participating students remained in the classroom. Teachers were not involved in the administration of the questionnaire. To adjust for lower reading competencies in the younger preadolescents ( $\leq 10$ years), I read aloud each question while children completed the survey. For the older children, I read aloud the instructions of the questionnaire to the groups, and participants read the individual items

\footnotetext{
${ }^{9}$ The class list was requested in order to match the parental questionnaire with the gender of the child (see Chapter 3). Class lists were only obtained for study 1.

${ }^{10}$ Because it was up to the schools to distribute the parental packages to the children, it is unknown if all packages were delivered to the children. Thus it is unknown if the return rate of $26.7 \%$ represents children not wanting to take part, parents not wanting their children to take part, or teachers not wanting their children to miss class. The return rate is likely to represent all of these factors, as well as unknown reasons for non participation.
} 
themselves. In all cases, I was available to assist participants with their responses when requested. At the end of each session, participating children were thanked for their time, any questions were answered, and children were given either a small piece of confectionary or a stationery item in appreciation for their time.

On completion of the study, both parents and schools were sent a debriefing sheet. Letters were sent to the parents of any children who scored at a concerning rate on the Children's Depression Inventory by a clinical psychologist supervising the project ${ }^{11}$. All children were informed of this possibility before completing the survey, and were given the option to not participate. There were no participants who decided not to participate based on the description of the study.

Measures

Stress $^{12}$. Stress was measured using a 6 -item scale constructed for the purposes of this study. Children responded on a 5-point Likert scale $(1=$ not true, $2=$ a little true, $3=$ somewhat true, $4=$ fairly true, $5=$ very true), and responses were summed to create a total stress score, with higher scores indicating greater stress. In this study, this scale exhibited excellent internal reliability $(\alpha=.91)$. Sense of Control $^{13}$. Sense of control was assessed using a 17 -item self-report scale taken from the Overall Sense of Control subscale from the Shapiro Control Inventory (Shapiro, 1994) and adapted by Weir and Jose (2006a) for use with children and adolescents. Nine items assess positive sense of control ("I have the right amount of self-control”), and 8 items assess negative sense of control ("I have less control than I used to"). Responses are given on a 5-point Likert scale (1 =

\footnotetext{
${ }^{11}$ Seven parents were informed in study 1 , three were informed in study 2 , and a further 9 were informed in study 3.

${ }^{12}$ See Appendix A for the Global Stress Scale

${ }^{13}$ See Appendix B for the Overall Sense of Control Scale
} 
never, $2=$ rarely, $3=$ sometimes, $4=$ usually, $5=$ always), where children and adolescents report how much control they feel they have over their lives in general. In this sample, the scale exhibited excellent internal reliability $(\alpha=.84)$.

$$
\text { Rumination }{ }^{14} \text {. Participants completed a shortened version (10 items) of the }
$$

ruminative response subscale from the Response Styles Questionnaire (RSQ;

Nolen-Hoeksema et al., 1993) ${ }^{15}$. The RSQ assesses an individual's tendency to respond to depressed mood by ruminating about their symptoms and/or the causes and consequences of depressive symptoms. Responses are given on a 5-point Likert scale $(1=$ never to $5=$ always $)$ with higher scores indicating higher levels of ruminative coping. The RSQ has demonstrated good internal reliability (Butler \& Nolen-Hoeksema, 1994), good test-retest reliability across time (Nolen-Hoeksema et al., 1994), and good predictive validity of depressive symptoms (Just \& Alloy, 1997; Nolen-Hoeksema, 2000). In this sample, the shortened scale had high internal reliability $(\alpha=.89)$.

\section{Depression $^{16}$. The Children's Depression Inventory (CDI; Kovacs, 1985;}

1992) is a 27-item pen-and-paper measure designed to assess the severity of depressive symptoms in children and adolescents aged 7-17 years. The CDI is not a diagnostic tool, but rather assesses the frequency and severity of depressive symptoms. The CDI is structured so that each of the 27 items is composed of 3 sentences ("I like myself”, "I do not like myself”, "I hate myself”), and within each item participants choose the one sentence that best represents how they have felt

\footnotetext{
${ }^{14}$ See Appendix C for the Response Styles Questionnaire used in this study.

${ }^{15}$ The 10 RRS items used in this study were the items that contributed most to the internal reliability of the RRS scale used in Jose and Brown (2007). The RRS was shortened because of time restrictions in data collection in the current study.

${ }^{16}$ See Appendix D for the Children's Depression Inventory
} 
over the past two weeks. Items are scored on a three-point scale: $0=$ no symptoms, $1=$ mild symptoms and $2=$ severe symptoms, with overall scores ranging between 0-54. A score above 20 indicates significantly depressed mood (Kovacs, 1992). The CDI has good internal reliability (Saylor, Finch, Spirito, \& Bennet, 1984), and is a valid measure of depressive symptoms (Kovacs, 1985). In this study, the scale exhibited excellent internal reliability $(\alpha=.90)$.

\section{Results}

Analytic Strategy

Recall there were five goals of this chapter. The first goal was to explore the developmental trends of rumination and depression in preadolescence, thus, gender and age differences on each variable were tested using a multivariate analysis of variance. The second and third goals relate to whether vulnerability factors (rumination and sense of control) influence the relationship between stress and depression. To this end, I tested whether rumination and sense of control acted as moderators or mediators of the relationship between stress and depression. The fourth goal, to explore the relationship between rumination and sense of control, was achieved partly through the moderation analyses, where rumination and sense of control were allowed to interact to predict depression. The relationship between rumination and sense of control was tested further using structural equation modeling; it was tested whether or not sense of control mediated the relationship between rumination and depressive symptoms. And last, also using structural equation modeling, the effects of age and gender on the interrelationships among stress, sense of control, rumination, and depressive symptoms were explored. Developmental Trends of Stress, Sense of Control, Rumination, and Depressive Symptoms 
A 2 (gender: male, female) x 5 (age: 9, 10, 11, 12, 13 year groups)

multivariate analysis of variance was conducted to test for the effects of gender and age on stress, rumination, sense of control, and depressive symptoms. Multivariate results indicated a significant multivariate effect of gender, $F(4,297)=2.58, p<$ $.05, \eta^{2}=.03$, and a marginally significant multivariate age $\mathrm{x}$ gender interaction ${ }^{17}$, $F(16,1200)=1.52, p=.08, \eta^{2}=.02$. The multivariate effect of age was not significant, $F(16,1200)=1.47, p=.10, \eta^{2}=.01$. Based on these results, univariate results were examined for the main effect of gender and the age by gender interaction, however univariate effects for age were not interpreted.

Univariate effects for gender indicated that males $(M=14.27$, S.D. $=6.42)$ and females $(M=14.79$, S.D. $=6.54)$ reported similar levels of stress, $F(1,300)=$ $.74, p=.39$, partial $\eta^{2}<.01$. Likewise, the difference between males $(M=68.29$, S.D. $=9.79)$ and females $(M=66.90$, S.D. $=9.17)$ on perceived control was not significant, $F(1,300)=1.01, p=.32$, partial $\eta^{2}<.01$. The difference between males $(M=24.84$, S.D. $=8.35)$ and females $(M=25.44$, S.D. $=8.31)$ on rumination was marginal, $F(1,300)=2.83, p=.09$, partial $\eta^{2}<.01$. And last, females $(M=10.89$, S.D. $=8.48)$ reported significantly more depressed mood than males $(M=8.98$, S.D. $=7.72), F(1,300)=6.39, p=.01$, partial $\eta^{2}=.02$ (Figure 2.1).

Focused $t$ tests were conducted on the depressive symptoms to examine the specific age that the gender difference became apparent. Males and females reported similar levels of depressive symptoms across the ages 9-12 years old, however, at

\footnotetext{
${ }^{17}$ Throughout this thesis, any $p$ value between .05 and .09 will be interpreted as marginally significant; $p$ values less than .05 will be interpreted as statistically significant, and $p$ values of .10 and above will be interpreted as statistically non-significant.
} 
age 13 , females $(M=11.25$, S.D. $=7.58)$ reported significantly more depressive symptoms than males, $(M=6.43$, S.D. $=4.13), t(28)=2.65, p=.01$, Cohen's $d$ $=.77$.

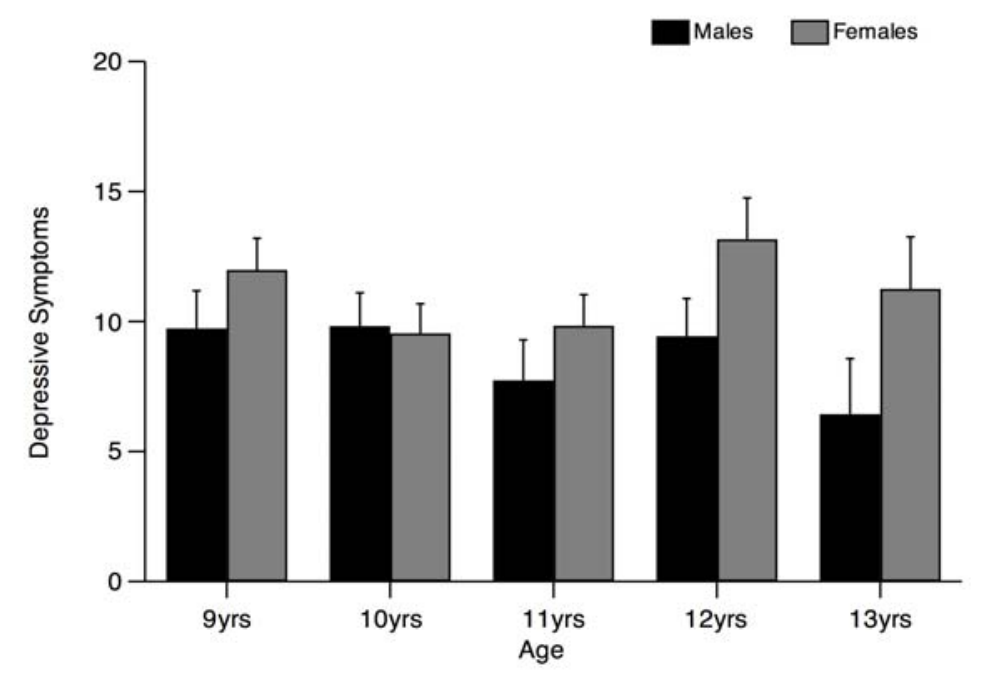

Figure 2.1. Depressive symptoms reported by males and females aged 9-13 years old.

Univariate effects for the age by gender interaction indicated that the age by gender interactions for stress, $F(1,300)=.78, p=.54$, partial $\eta^{2} \leq .01$; sense of control, $F(4,300)=.34, p=.84$, partial $\eta^{2}<.01$; and depression, $F(1,300)=$ $.79, p=.53$, partial $\eta^{2} \leq .01$, were not significant. Only the age by gender interaction for rumination was marginally significant, $F(1,300)=2.25, p=.06$, partial $\eta^{2}=.03$. A trend emerged where older females reported higher levels of rumination than older males (Figure 2.2). Focused $t$ test's indicated that females (M $=30.06$, S.D. $=8.20)$ reported higher levels of rumination than males $(\mathrm{M}=$ 22.57, S.D $=7.14$ ) only at age 13 years, $t(28)=2.65, p=.01$, Cohen's $d=.30$. 


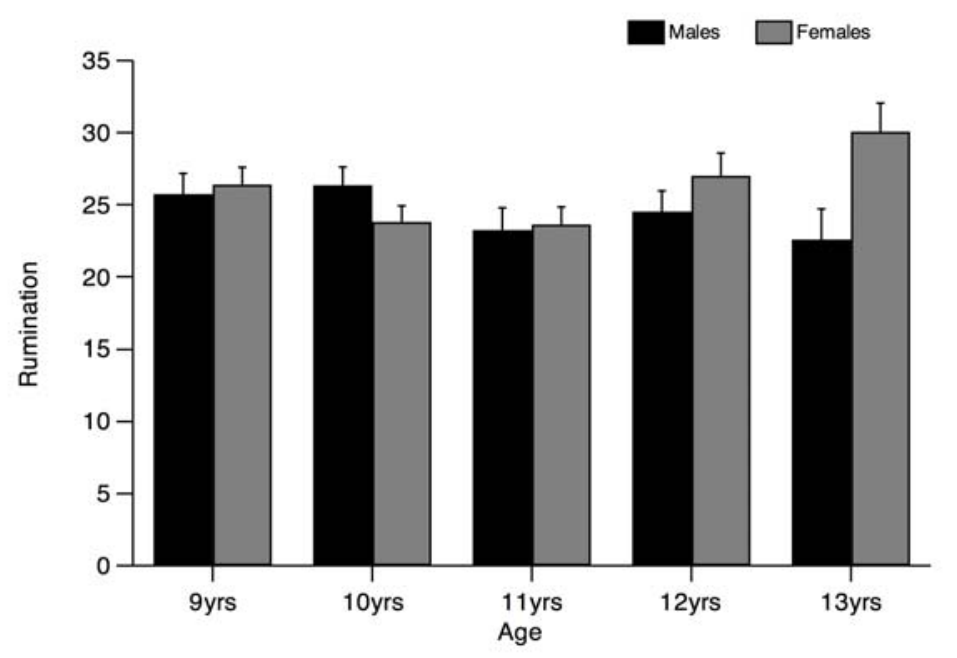

Figure 2.2. Rumination reported by males and females aged 9-13 years old.

Relationships Among Stress, Rumination, Sense of Control, and Depressive Symptoms.

Correlations. As illustrated in Table 2.1, higher self-reported stress and rumination was associated with heightened depressed mood. Lower self reported sense of control was associated with higher self reported depressed mood. Higher self reported levels of rumination were associated with more self-reported stress and a reduced sense of control over one's life. In sum, all variables were related to depressed mood in the predicted fashion.

Table 2.1. Correlations among Stress, Rumination, Sense of Control, and Depression

\begin{tabular}{lccc}
\hline & Stress & Rumination & Control \\
\hline Rumination & $.68 * * *$ & & \\
Control & $-.52 * * *$ & $-.43 * * *$ & \\
Depression & $.73 * * *$ & $.57 * * *$ & $-.62 * * *$ \\
\hline$* * *=p<.001$ & &
\end{tabular}

Moderation Analyses

A moderating variable is one where the impact of one variable (stress) on the outcome variable (depression) is influenced by the level of another variable (in this case either rumination or perceived control); moderating variables can either 
exacerbate or buffer against depressive symptoms (Baron \& Kenny, 1986; Holmbeck, 1997). For example, perceived control could be conceptualized as moderating the relationship between stress and depressive symptoms if stress was more highly associated with depressive symptoms when an individual felt they had little control over their life. Based on a diathesis-stress model of depression, high rumination and low perceived control should intensify the impact of stress on depression.

I conducted one moderation analysis to test whether the relationship between stress and depression was intensified by rumination and reduced by sense of control. Rather than conducting two moderation analyses (one for rumination and another for perceived control), a single moderation analysis was conducted to allow the theoretically interesting interaction of perceived control and rumination to impact upon the relationship between stress and depression. To reduce multicollinearity, all predictor variables were centered prior to creating the interaction terms (Howell, 2002). Following the recommendations of Holmbeck (1997), stress was entered in the first step of the hierarchical regression. The main effects of the moderators were entered during the second step (rumination, sense of control). The two-way interactions were entered in the third step (stress $\mathrm{x}$ rumination, stress $\mathrm{x}$ control, rumination $\mathrm{x}$ control). The three-way interaction was entered in the fourth step (stress x rumination x control). Results are presented in Table 2.2.

In the first step, stress was found to be a significant predictor, explaining over half of the variance in depressive symptoms. In step two, sense of control but not rumination significantly predicted depressive symptoms. Contrary to expectations, none of the neither two- nor three-way interactions were significant. In sum, neither 
rumination nor perceived control moderated the relationship between stress and depressive symptoms.

Table 2.2. Summary of Results of Rumination and Sense of Control Moderating the Relationship Between Stress and Depression

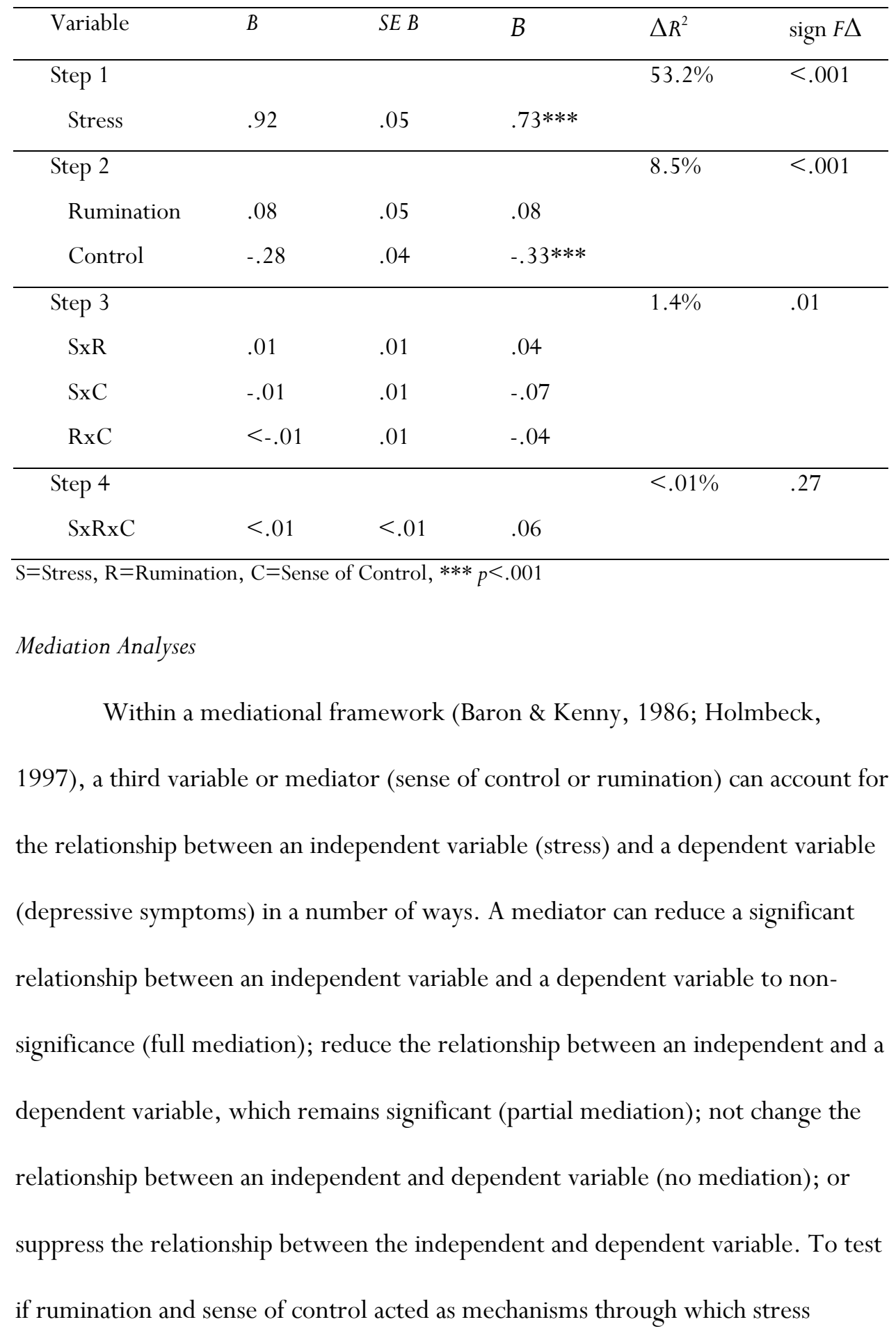


predicted depressive symptoms, I conducted two mediational analyses following the recommendations of Baron and Kenny using MedGraph (Jose, 2003). All variables were significantly correlated (Table 2.1), thus meeting the preconditions of mediation (Baron and Kenny).

Does rumination mediate the relationship between stress and depressive symptoms?

Figure 2.3 illustrates the mediational model. The values not in parenthesis represent zero order correlations, and the values in parenthesis represent the standardized beta weights after all variables are included in the analysis. The direct path from stress to depression remained significant after rumination was included in the analysis, however the basic relationship was significantly reduced, Sobel's $z=2.38$, $p=.02$. Thus, rumination partially mediated the relationship between stress and depressive symptoms. The mediating path (indirect path) from stress to depressive symptoms via rumination was also significant (.09); however the direct path (.64) from stress to depression was over 7 times as strong as this indirect path ${ }^{18}$.

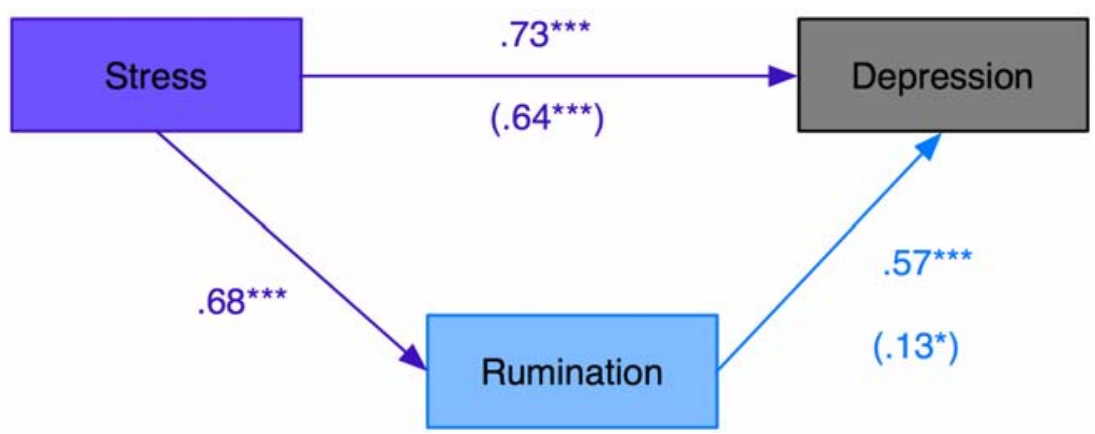

Figure 2.3. Rumination as a mediator between stress and depression

Does sense of control mediate the relationship between stress and depressive symptoms? The second mediation model tested whether perceived control mediated the relationship between stress and depression. The relationship

\footnotetext{
${ }^{18}$ The indirect path was calculated by multiplying each coefficient's value involved in the pathway (Schumacker \& Lomax, 1996). For example, the indirect path was calculated as $.68 \times .13=.09$.
} 
between stress and depression remained significant when sense of control was included into the model, yet the direct path was significantly reduced, Sobel's z $=$ $6.40, \mathrm{p}<.001$ (Figure 2.4). Thus, sense of control also partially mediated the relationship between stress and depressive symptoms. Again, the direct effect of stress on depression (.56) was stronger than the indirect path through sense of control (.17).

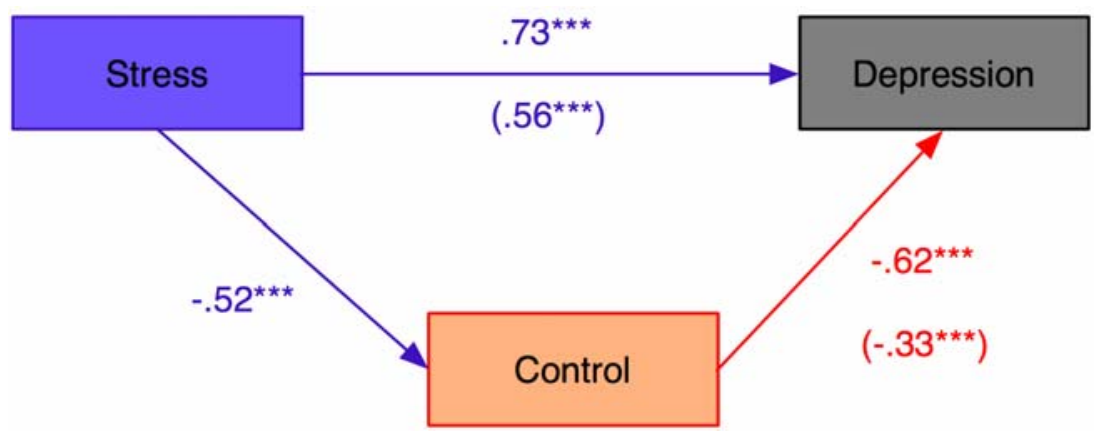

Figure 2.4 Sense of control as a mediator between stress and depression.

Comparative strengths of rumination and sense of control as mediators. For two reasons, structural equation modeling was employed to test mediation more thoroughly. First, employing structural equation modeling allowed for the two mediation paths to be examined simultaneously. Assessing mediation paths simultaneously allowed for the relative strengths of rumination and perceived control to be directly compared. In other words, I was able to assess whether rumination or perceived control more powerfully mediated the relationship between stress and depression. I was interested in whether the previously significant indirect effects would remain significant if both mediators were competing to explain variance in depressive symptoms.

Second, utilizing structural equation modeling allows for model fit indices to be considered. Several fit indices were considered to determine whether the 
proposed mediation models fit these data well. First, I used the ratio of the chisquare value divided by the degrees of freedom; a ratio of less than 3 for $\chi^{2} / d f$ is considered appropriate (Kline, 1998). Second, both the Goodness of Fit Index (GFI) and the Adjusted Goodness of Fit Index (AGFI) were used to assess the proportion of observed variance the model explained; values above 0.90 indicate an adequate fit (Kline). And third, two indices of residual fit were used. First, the standardized Root Mean Squared Residual (sRMR) was used; values below .08 considered acceptable (Hu \& Bentler, 1998), and a value of zero reflects a perfect fit (Kline). Second, the Root Mean Square Error of Approximation (RMSEA), which estimates the lack of fit of a given model compared to a saturated model, was used; this also reflects a perfect fit when equal to zero, with values under .06 indicating an appropriate fit (Hu \& Bentler).

The initial model (Figure 2.5) tested was a fully saturated model $(d f=0)$. This initial model involved the direct path from stress to depressive symptoms and two indirect paths to depressive symptoms: first from stress through rumination, and second, from stress through sense of control. Results indicated that perceived control mediated the relationship between stress and depressive symptoms, but rumination did not. Specifically, stress predicted depressive symptoms directly and indirectly $(\beta=.17)$ by passing through perceived control. It is notable that the indirect path from rumination to depressive symptoms reduced to become marginally significant $(p=.09)$, suggesting that rumination was not as powerful a mediator as sense of control for explaining the effect of stress on depressive symptoms. 
It is important to note that the $\chi^{2} / d f$ ratio and the RMSEA of this model fell

below acceptable standards, $\chi^{2}(1)=4.49, p=.03, G F I=.99, A G F I=.93, \mathrm{sRMR}=$ $.03, R M S E A=.11$. Furthermore, removing the non-significant link from rumination to depressive symptoms in this model did not improve the fit indices to acceptable levels across the board, $\chi^{2} / d f=3.65, G F I=.99, A G F I=.94, \mathrm{~s} R M R=$ .03, RMSEA $=.09$.

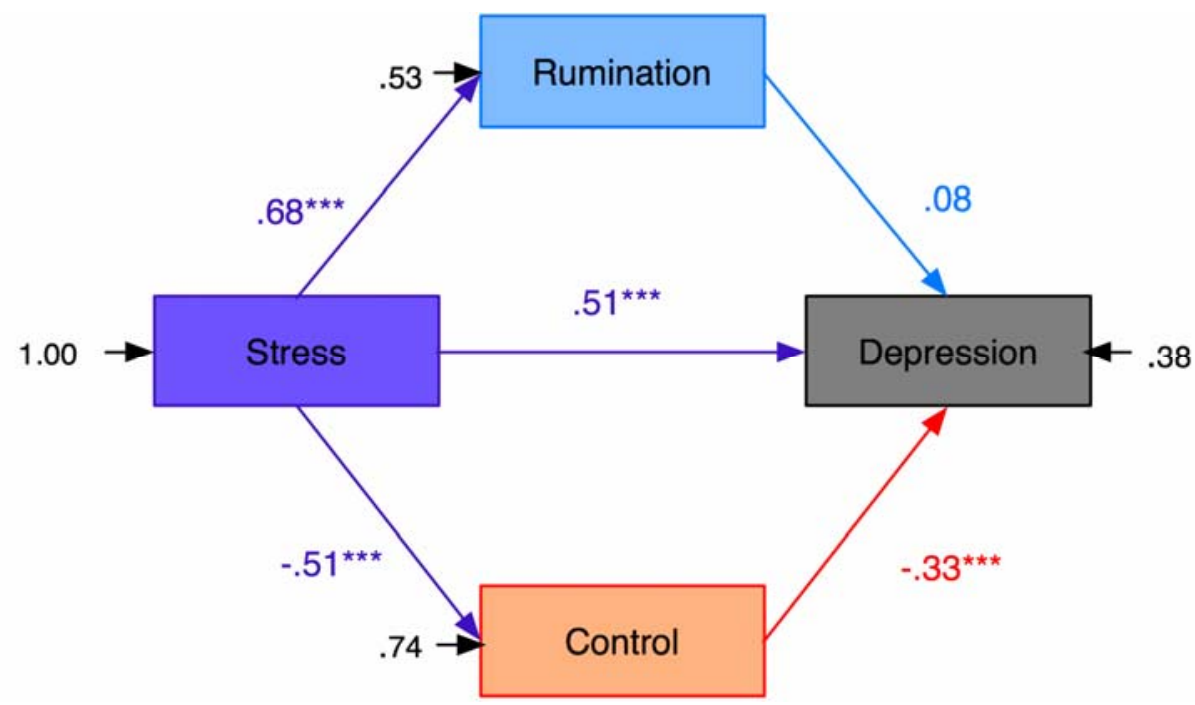

Figure 2.5. Rumination and sense of control as simultaneous mediators between stress and depressive symptoms

In an attempt to improve the model fit indices, I reanalyzed these data with the rumination to depressive symptoms path removed, (Kline, 2005) and based on modification indices and theory, an exploratory path from rumination to sense of control was inserted. This additional path is consistent with previous suggestions that hopelessness mediates the relationship between rumination and depression (Abela et al., 2002; Wänke \& Schmid, 1996). This model (Figure 2.6) fit these data well, $\chi^{2} / d f=2.79, G F I=1.0, A G F I=.96, \mathrm{~s} R M R=.01, R M S E A=.08$. Stress predicted depressive symptoms directly, and also indirectly, first by predicting a 
lowered sense of control $(\beta=.14)$, and second by predicting rumination, which then in turn predicted a lowered sense of control $(\beta=.03)$.

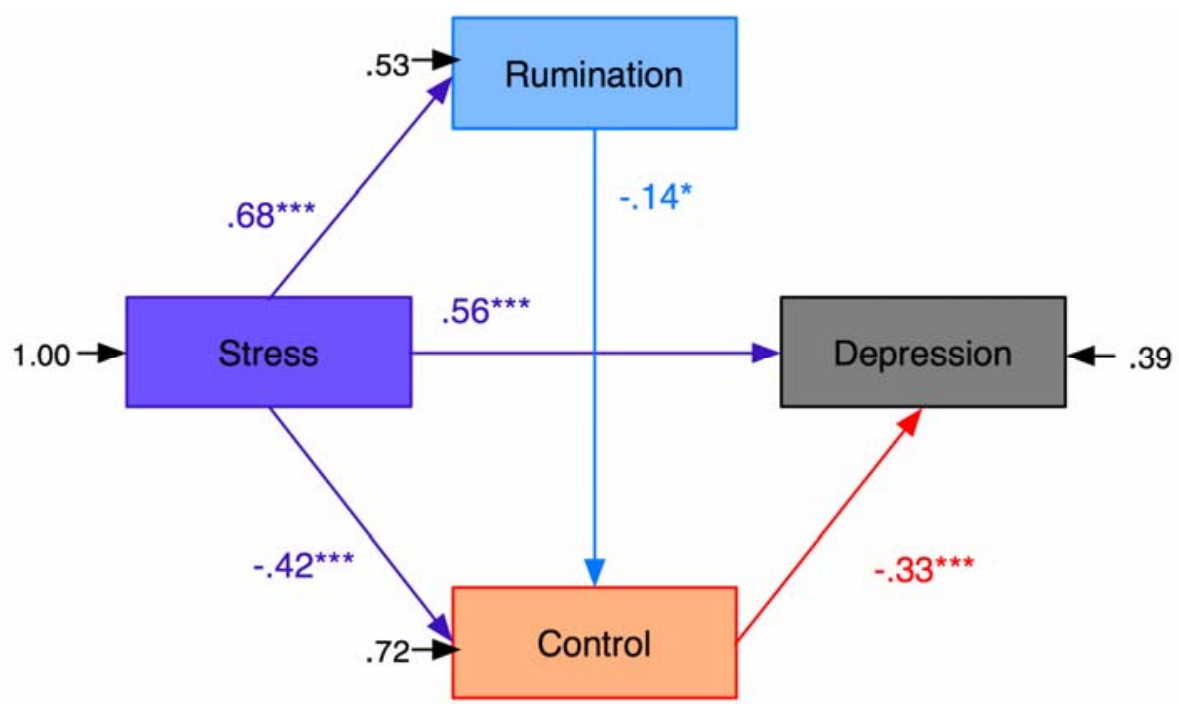

Figure 2.6. Pruned structural mediational model where rumination and sense of control mediate the relationship between stress and depression.

To explore if this final model was moderated by age or gender (i.e., moderated mediation), these data was reanalyzed separately for males and females, and for younger (9-10 years) and older preadolescents (11-13 years). For both the male and female models, the same pattern of results was obtained. Specifically, there were significant direct paths from stress to depressive symptoms (male: $\beta=$ $.57, p<.001$; female: $\beta=.56, p<.001)$, and indirect paths through lowered perceived control to depressive symptoms; the size of the indirect paths were .21 for males and .17 for females. However, a difference with the model for the entire sample was obtained in that the link between rumination and perceived control was not significant for either males $(\beta=-.14, p=.18)$ or females $(\beta=-.14, p=.11)$. The fit indices were acceptable for both males, $\chi^{2} / d f=1.87, G F I=.99, A G F I=$ 
.93, $\mathrm{sRMR}=.02, \mathrm{RMSEA}=.08$, and females, $\chi^{2} / \mathrm{df}=1.20, \mathrm{GFI}=1.00, \mathrm{AGFI}=$

$.97, \mathrm{sRMR}=.01, \mathrm{RMSEA}=.03$.

With regards to age, stress predicted depressive symptoms directly for both younger $(\beta=.57, p<.001)$ and older $(\beta=.55, p<.001)$ preadolescents. Stress also predicted depressive symptoms indirectly by passing through sense of control; the size of the indirect effects were -.20 for younger and -.10 for older preadolescents. Finally, the link from rumination to perceived control was nonsignificant for younger preadolescents $(\beta=-.11, p=.23)$, but marginally significant for older preadolescents $(\beta=-.19, p=.057)$. Consequently, rumination did not predict depressive symptoms for younger preadolescents, however rumination weakly predicted depressive symptoms by way of perceived control for older preadolescents. The indirect effect of stress through rumination and perceived control to depressive symptoms for older preadolescents was small (.04). This modified model fit these data of the older preadolescents group very well, $\chi^{2} / d f=$ $.54, G F I=1.00, A G F I=.98, \mathrm{~s} R M R=.01, R M S E A<.01$, but fell below acceptable standards on the RMSEA for younger preadolescents, $\chi^{2} / d f=2.48, G F I=.99$, $A G F I=.92, \mathrm{sRMR}=.02, \mathrm{RMSEA}=.09$. In summary, the interrelationships were similar for males and females, however the interrelationships became slightly more stable in older preadolescents.

\section{Discussion}

The current study examined the interrelationships among stress, rumination, sense of control, and depressive symptoms in a preadolescent sample. There were five major objectives of the current study, each of which will be 
discussed in turn. First, it was predicted that the gender difference in rumination would precede the gender difference in depressive symptoms. Contrary to expectations, the gender difference in rumination occurred simultaneously with the gender difference in depressive symptoms. That is, females reported both more rumination and depressive symptoms than males at age 13. Being that the gender difference in rumination was not evident prior to the gender difference in depressive symptoms, the assertion that females enter adolescence with greater vulnerability for depression was not supported (Nolen-Hoeksema, 1994). Because of the use of concurrent data used in the current sample, it is not possible to test whether females begin ruminating because of their depressive symptoms, or if females become more depressed because of their rumination.

The remaining goals of the current study related to how the interrelationships among stress, rumination, and sense of control predict depressive symptoms. A stress-diathesis model of depression during adolescence (NolenHoeksema, 1994; Nolen-Hoeksema \& Girgus, 1994); where rumination and sense of control were stipulated as vulnerability factors was tested. Results from moderation analyses indicated that stress did not interact with either rumination or perceived control to predict depressive symptoms. Instead, rumination and perceived control were found to mediate the relationship between stress and depressive symptoms. Conceptually, this means that rumination and sense of control explain significant portions of the association between stress and depression. These results support recent trends in the conceptualization of diathesis-stress models of psychopathology, which have moved towards viewing vulnerability 
factors as mediators rather than moderators of stressful life events and negative outcomes (Cole \& Turner, 1993; Gibb \& Alloy, 2006).

Although rumination was found to be a mediator of the relationship between stress and depression, its strength of effect was less than that of sense of control. When the effect of rumination was tested in isolation of additional mediating variables, rumination partially mediated the relationship between stress and depression. Yet, when rumination was analyzed simultaneously with sense of control, rumination no longer functioned as a significant mediator. This result suggests that the association between rumination and depressive symptoms in preadolescent populations may be the product of the associations among rumination and additional variables (the third variable phenomenon). One such third variable that may explain the association between rumination and depressive symptoms appears to be perceived control. In the modified full mediation model (Figure 2.6), rumination was allowed to influence depressive symptoms through the indirect path involving perceived control; this resulted in a significant, albeit small, indirect pathway to depression, supporting previous authors' assertions that the relationship between rumination and depression may be mediated by hopelessness (perceived control) (Abela et al., 2002). Thus, the results of the current study suggest that rumination does mediate the relationship between stress and depressive symptoms, but rumination's effect may operate through a more circuitous mediation pathway, i.e., through perceived sense of control.

Although the above results appear to not provide supportive evidence for RST, partial support was provided through the finding that gender did not moderate the interrelationships among stress, rumination, and depression. RST asserts that 
the development of depressed mood is similar for males and females. Supporting such a view, in this study, the interrelationships among stress, rumination, perceived control, and depressive symptoms were found to be similar for males and females. Thus, the pathway to depressive symptoms did not seem to be genderspecific in this preadolescent sample.

Although pathways to depressive symptoms were similar for males and females, the relationship between rumination and perceived control was somewhat influenced by age. Specifically, rumination was not significantly associated with perceived control for younger preadolescents (9-10 years); however rumination was related to perceived control for the older preadolescents (11-13 years). In a recent review of the relationship between self-focused attention and maladjustment, Mor and Winquist (2002) found that the relationship between rumination and depression was stronger for adults than adolescents. Mor and Winquist suggested this result may be obtained because with age, adolescents are less likely to inflate their self-evaluations (Ruble, Eisenberg, \& Higgins, 1994), and at the same time they also develop more complex self-schemas (Harter, 1999). Supporting such an assertion, our results suggest that with age, the interrelationships among response styles and personality variables become increasingly consolidated and predictable. For example, a more stable relationship between perceived control and rumination appears to begin at around age 11 . This consolidation may occur because with age, adolescents are more capable of incorporating different aspects of their self concept, for example, "I am stressed"; "I am worried about the future”; "I feel sad”, into a more abstract self concept, such as "I feel distressed" (Harter). Such consolidation may help explain the increasing interrelationship between sense of control and 
rumination; however, future research is required to explore more fully this possibility.

In combination, these results did not provide strong support for the application of RST to a preadolescent sample. First, the gender difference in rumination occurred in unison with the gender difference in depressive symptoms. Second, rumination mediated the relationship between stress and depression, but did not moderate this relationship. Third, RST was not as predictive as sense of control of depressive symptoms, suggesting that the effect of rumination on depressive symptoms may be, in part, because of a third variable phenomenon. Fourth, rumination and sense of control did not interact to predict depressive symptoms; instead, rumination appeared to predict depressive symptoms by predicting decreases in perceived control. And last, the interrelationships among stress, rumination, sense of control, and depressive symptoms were similar for males and females; however the relationship between vulnerability factors appeared to stabilize somewhat in older preadolescents.

In study 2 (Chapter 5) these results are built upon, and two of the current study's limitations are addressed. First, given that the current study used concurrent data the causal pathway between stress, sense of control, rumination, and depressive symptoms could not be estimated. In study 2 data is collected across 10 weeks, and the direction of effect between risk, vulnerability, and maladjustment is estimated. Second, the current sample consisted of preadolescents only and therefore the applicability of RST to adolescence could not be assessed. Because adolescence is often conceptualized as the age at which gender differences in risk, vulnerability, and maladjustment emerge (Allgood-Merten et al., 1990; Twenge \& 
Nolen-Hoeksema, 2002), involving both preadolescents and adolescents in a sample is critical for a full test of RST. 


\section{Chapter 3: Parental and Peer Influences in Developing a Ruminative Response Style During Preadolescence}

Although rumination appears to become more prevalent among females during adolescence, why this happens is not yet well understood. The reasons behind why females begin ruminating more than males during adolescence are the focus of the current chapter. Nolen-Hoeksema (1987; 1991) has suggested a number of ways parents may influence the use of rumination in their children. First, she has suggested that parents may model a ruminative response style. Support for this assertion comes from findings that children of depressed parents are more likely to use passive coping strategies, and are more likely to experience depression than children with non-depressed parents (Hammen, 2005). Second, because parents tend to discourage feminine typed behaviours among males, parents may discourage rumination more among males than females (Nolen-Hoeksema, 1991). In the present study, I tested whether parents (of participants described in chapter 2) encourage rumination in females more than males when experiencing hypothetically distressing events.

Peers may also play a role in the development of a ruminative response style. The gender-intensification hypothesis (Hill \& Lynch, 1983; Wichstrøm, 1999) asserts that during adolescence, females are expected to display increasingly stereotypical feminine behaviours (such as rumination) and males are expected to display increasingly stereotypical masculine behaviours. Thus, during early and middle adolescence, there is heightened adherence to gender-related norms, as well as heightened discriminatory behaviours towards adolescents who do not conform to stereotypical behaviours (Lobel, Nov-Krispin, Schiller, Lobel, \& Feldman, 2004). Because adolescence is characterized as a period when peers expect others to 
adhere to stereotypical response strategies, females may be expected to ruminate more than males during or directly prior to this period. Thus, the study presented here also tests whether preadolescents expect other preadolescents to respond to stressful events with gender-specific response strategies.

This study also explored how parental encouragement and peer expectations relate to self-reported levels of rumination. Very few studies have empirically examined the relationship between expectations of others and self reported rumination. In one of the few studies from the adult literature, Strauss et al. (1997) found that not only did females self-report ruminating more than males, but regardless of gender of perceiver, female protagonists (in a vignette) were expected to ruminate more than males. Furthermore, this endorsement of stereotypical gender-related response styles was evident even when the rater knew the protagonist, for example when the protagonist was the rater's roommate. Thus, there is tentative evidence that both males and females expect females to ruminate more than males, and that stereotypes match actual self-reported levels of rumination.

There is a general lack of research evidence in the child and adolescent literature. One exception is Broderick and Korteland's (2002) study, which examined whether or not adolescents expected male and female peers to respond to distress in stereotypical ways. In this study, adolescents read vignettes about male and female adolescents in stressful situations. The protagonists either ruminated or distracted in response to the situation. As predicted by RST, adolescents expected males to distract more than females and females to ruminate more than males. Furthermore, developmental effects were evident; stereotypical responses were 
most typically expected by older adolescents ( $8^{\text {th }}$ graders). Broderick and Korteland concluded that gender-based implicit beliefs were generalized for males and females during adolescence. Such increasingly constraining stereotypical expectations for males and females throughout adolescence may help explain why females begin ruminating more than males during this period.

To date, the study by Broderick and Korteland (2002) is one of the few empirical investigations examining the stereotypes that adolescents hold about how males and females typically respond to distressing situations, regarding rumination. Thus, Broderick and Korteland's research represents an important extension of current theorizing on the origins of rumination because most theories have focused on the socialization practices of parents (Nolen-Hoeksema, 1987; 1991) even though the peer environment becomes increasingly important during adolescence. The influence of peer groups is especially potent when referring to gender role socialization (Hill \& Lynch, 1983). Unfortunately, Broderick and Korteland did not compare how the expectations of gender-specific response styles related to self reported levels of rumination.

The current study aims to address this limitation of existing research by presenting parents and adolescents with vignettes of male and female preadolescents experiencing school-, social-, and family-related stressors. Data were collected from parents to determine whether they expressed encouragement (or not) for their child to ruminate in response to each stressor. Further, data were also collected from adolescents who reported whether or not they expected others to respond to the situations by ruminating. Parental encouragement and adolescents expectations of rumination were considered in light of the adolescents' actual self- 
reported rumination. First, it was predicted that parents would report encouraging their daughters to ruminate more than their sons. Second, it was predicted that adolescents would ruminate more if their parents encouraged them to ruminate in response to distressing situations. Third, regardless of an adolescents own gender, it was predicted that female protagonists would be expected to respond to distress with rumination more than male protagonists. And fourth, it was predicted that adolescents' expectations of the protagonists level of rumination would be associated with higher self-reported rumination, namely, if an adolescent expects others will ruminate, he or she will report higher levels of self-reported rumination.

\section{Method}

Participants and Procedure

Parents. Three hundred and seventeen parents initially returned a parental questionnaire. However 38 parents completed the survey incorrectly (they made one rather than five responses in the coping style section) and were deleted from the study. Of the remaining 279 parents, 218 were able to be matched with children's questionnaires ${ }^{19}$. Parents were, on average, 40.85 years old (S.D. = 5.27; range $=26-55$ years old); six parents did not report their age. Participating parents were predominantly mothers (84\%). The majority of parents $(80 \%)$ were Pakeha/European New Zealander, 7.5\% were Maori, 2.5\% were Pacific Islanders, 4\% were Asian, 5\% classified their ethnicity as "Other", and 1\% did not provide information about their ethnicity.

\footnotetext{
${ }^{19}$ The main reason for non-matched parents was that children did not participate in the study, either because they were absent on the day of data collection, or they did not return their parental consent form; 19 parents returned their child's consent form with their parental questionnaire after child data had been collected.
} 
Preadolescents. Participating children were a subset of those described in Chapter 2. The sample was composed only of those children who completed all measures in the children's survey, and whose parents returned a completed parental questionnaire. The procedure for recruitment was previously reported in chapter 1 . Two hundred and eighteen preadolescents (95 males, 123 females) from 12 schools throughout central New Zealand participated in this study. All preadolescents completed the battery of measures in small groups in a quiet area designated by the school. Fifty-one participants (24 males, 27 females) were nine years old, 71 (32 males, 39 females) were 10 years old, 45 (16 males, 29 females) were 11 years old, and 51 (23 males, 28 females) were 12-13 years old. The majority of preadolescents were Pakeha/European New Zealanders (67\%), 11\% were Maori, 1\% was Pacific Islanders, 4\% were Asian, and 17\% classified their ethnicity as "Other".

\section{Preadolescent Questionnaire}

Scenarios $^{20}$. Nine scenarios were written for this study depicting preadolescents in distressing situations. These nine scenarios described three school, three family, and three social domains since these contexts represent the most common and important domains assessed in children and adolescents' lives (e.g. Broderick, 1998; Broderick \& Korteland, 2002). Scenarios in the school domain included a preadolescent who does badly in a test he or she studied hard for, is bored by schoolwork, and has a teacher picking on him or her. Scenarios in the family domain included a preadolescent who has a fight with his or her sibling, is blamed by his or her sibling for something he or she did not do, and hears his or her parents arguing. Scenarios in the social domain included a preadolescent who does

\footnotetext{
${ }^{20}$ An example of set of scenarios are presented in Appendix E.
} 
not get invited to a birthday party, is teased by other preadolescents, and has a fight with a close friend.

Scenarios were presented as cartoons rather than written vignettes because recent research has shown that cartoon vignettes increase interest from preadolescents, allow for a wider range of reading capabilities, and are a psychometrically valid alternative to written vignettes (Leff et al., 2006). The protagonist of the vignettes was depicted as an eleven year old in order to be close to all ages in the sample.

All preadolescents were presented with two versions of the cartoons, the 'self' and the 'other' conditions. In the 'self' condition, the gender of the protagonist was matched with the participant. Preadolescents were asked to indicate how they would personally respond to the situations. They were presented with five alternative styles of coping (problem solving, rumination, distraction, denial, and externalizing $)$ and asked to indicate on a 5 -point Likert scale $(1=$ not at all, $2=\mathrm{a}$ little, $3=$ some, $4=$ a fair amount, $5=$ constantly) how much they would utilize each coping style. Each response category was adapted to match the scenario. For example, in the 'had a fight with a close friend' scenario, the rumination item was "I think there must be something wrong with me or we wouldn't have fought"). Rumination items were adapted from the Response Style Questionnaire (NolenHoeksema et al., 1993). In this chapter, only the rumination items were examined; alternative items were presented to reduce the transparency of the study's main purpose.

In the 'other' condition, the protagonist was either "Sarah" (female condition) or "Andrew" (male condition). Preadolescents indicated how they 
thought the preadolescent would respond using the same items as described for the 'self' condition. Preadolescents only viewed 6 scenarios (3 'self', 3 “'other')

because of time restrictions in the administration of the questionnaire.

Counterbalancing. Various aspects of the scenarios were counterbalanced to reduce order effects. First, whether preadolescents were presented with the 'self' or 'other' condition first was counterbalanced. Second, the "other" condition needed further counterbalancing to reduce the effects that the gender of the protagonist might have on the results. There were 6 versions of the "other" conditions (see Table 3.1). These 6 "other" versions became 12 when counterbalanced for order of "self" vs. "other". Further, these 12 versions were doubled to 24 versions when creating both the male and female protagonists for the "self" condition. Across these 24 versions roughly equal numbers of sets of scenarios were completed. For males, the number of each version completed ranged between 6 and 12 , and for females, the number of each version completed ranged between 8 and 14. To test whether the version of the questionnaire had an effect on responses, a 2 (target: self, other) x 24 (version: 1 - 24) repeated measures ANOVA was conducted. Results indicated that there was no effect of version on responses, $F(23$, $194)=.49, p=.98$.

Table 3.1. Order of Scenarios Presented in Each of the Six Base Versions

\begin{tabular}{cccccccccc}
\hline Dom & Ord & Ver1 & Ver2 & Ord & Ver3 & Ver4 & Ord & Ver5 & Ver6 \\
\hline Sch & 1 & F & M & 3 & F & M & 2 & F & M \\
& & Test & Test & & Bor & Bor & & Teac & Teac \\
Fam & 2 & M & F & 1 & B & F & 3 & M & F \\
& & Sib & Sib & & Blam & Blam & & Par & Par \\
Soc & 3 & F & M & 2 & F & M & 1 & F & M \\
& & Bday & Bday & & Teas & Teas & & Fri & Fri \\
\hline
\end{tabular}

Note: Dom = Domain; Ord = Order; Ver = Version; $M=$ male protagonist; $F=$ female protagonist; Test $=$ does badly in a test; Sibl = fights with sibling; Bday $=$ not invited to birthday party; Bor = bored at school; Blam = blamed by sibling; Teas = teased by other children; Teac $=$ picked on by teacher; Par = hears parents fighting; Fri = fight with friend. 
Self-Reported Rumination. Preadolescents completed the 10 item reduced version of the Response Styles Questionnaire (RSQ; Nolen-Hoeksema et al., 1993), described in Chapter 2, which exhibited excellent internal reliability in this reduced sample $(\alpha=.89)$.

Parental Questionnaire

The parental questionnaire contained all nine scenarios in cartoon form (as opposed to 6 for the children) ${ }^{21}$. Parents were instructed to imagine that the protagonist was their child, and asked to indicate how they would encourage their child to respond in each situation. As with the preadolescent scenarios, parents were given five response options for how they would encourage their child to respond to each scenario. However, as mentioned above, only the rumination items were examined here. Three versions of the parental questionnaires were created. The three versions only differed in terms of the order of the scenarios (recall that parents saw all nine scenarios). The orders of the three versions were selected using random number tables. Two sets of these three versions were made; one with a female protagonist and another with a male protagonist. In all cases, the protagonist in each scenario matched the gender of the target child. Roughly equal numbers of the versions were completed by the parents. To check if version influenced responses, an ANOVA was conducted with 'version' as the independent variable, and 'total parental encouragement to ruminate' as the dependent variable. Results indicated that the version of the questionnaire had no effect on parental responses, $F(5,212)=.69, p=.63$.

\footnotetext{
${ }^{21}$ See Appendix F for an example set of parental scenarios
} 


\section{Results}

Parental Encouragement of Rumination Based on Children's Age and Gender

Responses were summed across the 9 scenarios to get a total parental score. A 2 (gender: male or female) x 4 (age: 9, 10, 11, or 12-13 years old) ANOVA was conducted on the dependent variable of parent-reported encouragement of rumination. Contrary to expectations, parents did not encourage rumination differently based on gender, $F(1,210)=1.94, p=.17$, partial $\eta^{2}=.01$, or age, $F(3,210)=1.13, p=.34$, partial $\eta^{2}=.02$. The age by gender interaction was also not significant, $F(3,210)=.71, p=.55$, partial $\eta^{2}=.01$ (See Figure 3.1).

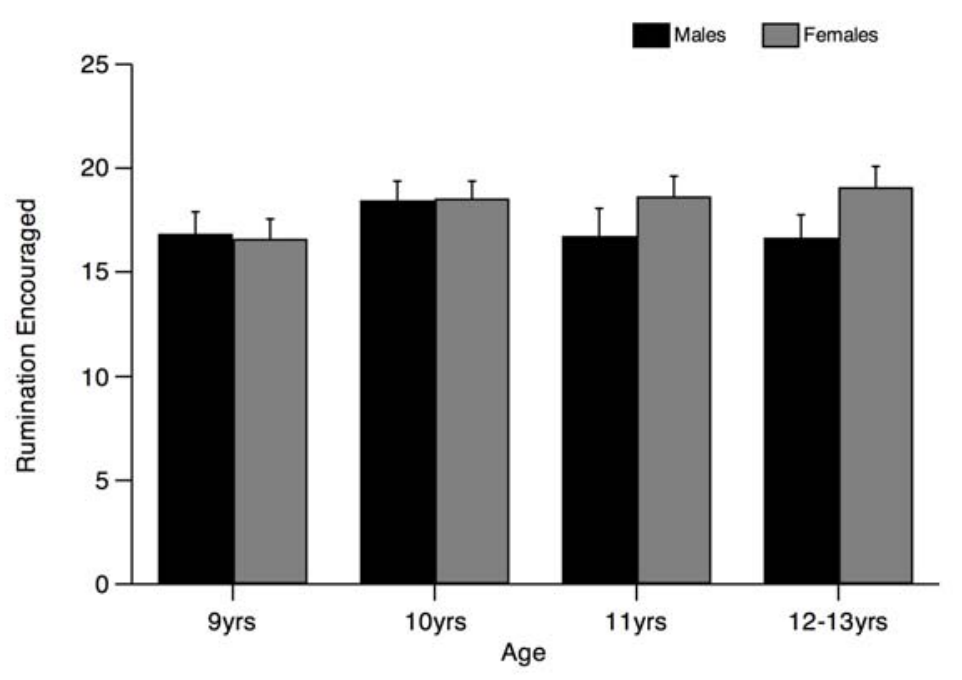

Figure 3.1. Parental encouragement of rumination for males and females aged 9-13 years.

To explore whether age or gender influenced parents' encouragement to ruminate in specific domains, a 2 (gender: male or female) x 3 (domain: school, family, or social) x 4 (age: 9, 10, 11, or 12-13 years old) repeated measures ANOVA was conducted. A significant within-subjects main effect of domain was found, $F(2,420)=90.06, p<.001$, partial $\eta^{2}=.30 ;$ parents encouraged rumination in response to school stressors most frequently, $(M=7.02, S D=2.48)$, 
followed by family $(M=5.68, S D=1.99)$, and lastly social domains $(M=5.12, S D$ $=2.09)$. Within-subject contrasts indicated that parental encouragement to ruminate differed significantly across all three domains. No other effects (the domain $\mathrm{x}$ gender, the domain $\mathrm{x}$ age, nor the domain $\mathrm{x}$ gender $\mathrm{x}$ age interactions) were significant, $F \mathrm{~s}=.08$ to $.74, p \mathrm{~s}=.62$ to $.98, \eta^{2} \mathrm{~s} \leq .01$. Likewise, none of the between-subjects effects-gender, age, or the gender x age interaction-was significant, $F_{\mathrm{s}}=.71$ to $1.94, \mathrm{ps}=.17$ to $.55, \eta^{2} \leq .01$.

In summary, preadolescents were not encouraged by parents to ruminate differently based on their age and gender. Instead, parents encouraged their children to ruminate differently based on the context of their stressors. That is, preadolescents were encouraged to ruminate most in response to school-related stressors, followed by family, and least for social stressors. Preadolescents Self Reported Levels of Rumination

To examine the developmental trends of rumination for males and females, rumination scores were summed across the three domains to create a total rumination score. A 2 (gender: male or female) x 4 (age: 9, 10, 11, or 12-13 years old) ANOVA was conducted to test for age and gender effects. Males and females reported similar levels of rumination, $F(1,210)=.06, p=.80, \eta^{2} \leq .01$. Similarly, about equal levels of rumination were reported regardless of age, $F(3,210)=1.46$, $p=.23, \eta^{2}=.02$. The age by gender interaction was also not significant, $F(3,210)$ $=.61, p=.61, \eta^{2} \leq .01$. Therefore, adolescents reported similar levels of rumination regardless of their age or gender. Descriptive statistics for males and females across the three domains are presented in Table 3.2. 
To explore whether age or gender influenced self-reported rumination in specific domains, a 2 (gender: male or female) x 3 (domain: school, family, or social) x 4 (age: 9, 10, 11, or 12-13 years old) repeated measures ANOVA was conducted. Preadolescents did not ruminate differentially by domain, $F(2,420)=$ $1.48, p=.23$, partial $\eta^{2}=.01$. No other effects (i.e., the domain $\mathrm{x}$ gender, the domain $\mathrm{x}$ age, nor the domain $\mathrm{x}$ gender $\mathrm{x}$ age interactions) were significant, $F \mathrm{~s}=$ .56 to $1.79, p s=.17$ to $.96, \eta^{2} \leq .01$. Likewise, none of the between-subjects effects (gender, age, or the gender $\mathrm{x}$ age interaction) were significant, $F \mathrm{~s}=.04$ to $1.43, p s=.24$ to $.85, \eta^{2} \leq .02$.

Table 3.2. Means and Standard Deviations for Male and Female Responses for Self and Other Conditions

\begin{tabular}{lcccccccc}
\hline & \multicolumn{2}{c}{ School } & \multicolumn{2}{c}{ Family } & \multicolumn{2}{c}{ Social } & \multicolumn{2}{c}{ Total } \\
& $M$ & $S D$ & $M$ & $S D$ & $M$ & $S D$ & $M$ & $S D$ \\
\hline Male S & 2.35 & 1.21 & 2.18 & 1.67 & 2.09 & 1.10 & 6.62 & 2.57 \\
Female S & 2.22 & 1.11 & 2.17 & 1.16 & 2.24 & 1.08 & 6.65 & 2.73 \\
\hline Male O & 2.41 & 1.06 & 2.58 & 1.17 & 3.35 & 1.22 & 7.33 & 2.49 \\
Female O & 2.55 & 1.09 & 2.42 & 1.63 & 3.31 & 1.15 & 7.28 & 2.54 \\
\hline
\end{tabular}

Note $\mathrm{S}=$ Self Condition; $\mathrm{O}=$ Other condition

The Relationship Between Parental Encouragement of Rumination and Self Reported

Rumination Among Preadolescents

It was predicted that preadolescents' actual levels of rumination would be associated with the degree of encouragement to ruminate by parents'. To examine this, parents' rumination encouragement scores were correlated with adolescents' scores using adolescents reported total scores, domain scores, and RSQ scores. Preadolescents' self-reported rumination was generally unrelated to parental encouragement of rumination whether rumination was measured using total scores, 
domain scores, or RSQ scores (Table 3.3). Thus, contrary to expectations, parental encouragement of rumination does not appear to be associated with rumination in preadolescents. Interestingly, preadolescents and parents were consistent in their self-reported levels of rumination (preadolescents) or their encouragement of rumination (parents) across the three domains.

Table 3.3. Correlations Among Parental Encouragement of Rumination and Child Self-Reported Rumination using Total Scores, Domain Scores, and RSQ Scores

\begin{tabular}{|c|c|c|c|c|c|c|c|c|}
\hline & RSQ & CTot & CSch & CFam & CSoc & PTot & PSch & PFam \\
\hline \multicolumn{9}{|l|}{ RSQ } \\
\hline CTot & $.58 * * *$ & & & & & & & \\
\hline CSch & $.42 * * *$ & $.77 * * *$ & & & & & & \\
\hline CFam & $.54 * * *$ & $.82 * * *$ & $.47 * * *$ & & & & & \\
\hline CSoc & $.41 * * *$ & $.75 * * *$ & $.32 * * *$ & $.46 * * *$ & & & & \\
\hline PTot & -.04 & .05 & .07 & .00 & .05 & & & \\
\hline PSch & -.05 & -.03 & .00 & -.07 & .00 & $.87 * * *$ & & \\
\hline PFam & -.06 & $.14 *$ & $.11 *$ & .06 & $.14 *$ & $.82 * * *$ & $.56 * * *$ & \\
\hline PSoc & .02 & .05 & .06 & .04 & .00 & $.84 * * *$ & $.59 * * *$ & $.56 * * *$ \\
\hline
\end{tabular}

$*=p<.05, * * *=p<.001$. CTot $=$ children's total rumination score across the domains; CSch $=$ children's rumination in school domain; CFam = children's rumination in family domain; CSoc $=$ children's rumination in the social domain; PTot = parents' total encouragement of rumination across the domains; PSch = parents' total encouragement of rumination in school domain; PFam = parents' encouragement of rumination in family domain; PSoc $=$ parents' encouragement of rumination in the social domain.

Next, I tested whether the relationship between parental encouragement and self-reported preadolescent rumination levels was dependent on the age or gender of the preadolescent. That is, was the relationship between parental encouragement of rumination and self-reported levels of rumination moderated by the age or gender of the preadolescent? Following the recommendations of Baron and Kenny (1986) and Holmbeck (1997), the main effects (total parental encouragement to rumination [PTotal], age, gender) were entered in the first step of the hierarchical regression, the two-way interactions (PTotal $\mathrm{x}$ age; PTotal $\mathrm{x}$ 
gender; age $\mathrm{x}$ gender) were entered in the second step, and the three-way interaction (PTotal $\mathrm{x}$ age $\mathrm{x}$ gender) was entered in the third step. Preadolescents' self-reported levels of rumination were measured using RSQ scores. None of the steps of the moderation analysis were significant. Furthermore, the Adjusted $R^{2}$ indicated that only $1 \%$ of variance in preadolescents' self-reported rumination was accounted for by the moderation analysis, thus beta weights were not interpreted. In sum, the relationship between parent's encouragement of rumination and preadolescents' self-reported rumination is not moderated by children's age and gender.

Preadolescents Expectations of Rumination among their Peers

It was predicted that preadolescents would expect female preadolescents to ruminate more than preadolescent males. To test this hypothesis, three 2 (gender of preadolescent: male or female) x 2 (gender of protagonist: male or female) ANOVAs were conducted, one for each domain. Three ANOVAs were performed rather than one repeated measures MANOVA because the gender of the protagonist varied depending on the version of the questionnaire. That is, the gender of the protagonist varied across domain, as described above, and it was thus unable to be tested using a repeated measures design. Analyses were not conducted by age due to small cell sizes. No significant effects of gender were found across the three scenarios, $F_{\mathrm{s}}=.12$ to $1.28, \mathrm{ps}=.26$ to $.91, \eta^{2}<.01$ nor were any of the gender by sex of protagonist interactions significant across the three scenarios, $F_{\mathrm{s}}=.61$ to $1.65, \mathrm{ps}=.20$ to $.43, \eta^{2} \leq .01$. For both the school and the family related scenarios, no significant effects of sex of the protagonist were evident, $F_{\mathrm{s}}=.29$ to $.36, p \mathrm{~s}=$ .51 to $.55, \eta^{2}<.01$. However a marginally significant effect of sex of protagonist 
was evident for the social scenario, $F(1,214)=3.25, p=.07, \eta^{2}=.02$. For both male and female participants, when the sex of the protagonist was different to their own sex, they expected the protagonist to ruminate marginally more $(M=2.21, S D$ $=.10)$ than if the sex of the protagonist was the same as their own $(M=2.46, S D=$ .10). Thus, contrary to predictions, preadolescents as a group did not expect female protagonists to ruminate more than male protagonists.

The Relationship Between Self Reported Rumination and Preadolescents Expectations of Their Peers Level of Rumination

Total 'self' and 'other' rumination scores were correlated to test whether preadolescents' self-reported levels of rumination and their expectation of others were related. It was predicted that adolescents would ruminate more if they expected others to ruminate, because peer groups become increasingly instrumental during adolescence (Hill \& Lynch, 1983). Supporting this prediction, there was a strong, positive correlation between self-reported rumination and how much preadolescents' thought others ruminated, $r(216)=.60, p<.001$. This correlation was similar for both males, $r(93)=.59, p<.001$, and females, $r(121)=.60, p<$ .001 Analyzed separately by domain, self-reported levels of rumination were related to their expectations of other preadolescents' in school, $r(216)=.40, p<$ .001 , family, $r(216)=.35, p<.001$, and social, $r(216)=.29, p<.001$, domains. Thus, if preadolescents' ruminated, they expected other preadolescents' to ruminate as well and vice versa, regardless if the preadolescent was male or female.

\section{Discussion}

Previous research has indicated that females are more ruminative than males during adolescence, particularly from 13 years old (Jose \& Brown, 2007; 
Schwartz \& Koenig, 1996; Ziegert \& Kistner, 2002). However, very little research has examined how such response styles develop. In this chapter, potential social influences on developing a ruminative response style were examined. By and large, current theories as to why females start ruminating more than males adopt a social learning theory perspective (Bandura, 1986). That is, females are thought to ruminate more than males in part because their parents model ruminative tendencies or because passivity is disproportionately encouraged and expected in females (Nolen-Hoeksema, 1987; 1991). This study provided preliminary research testing these assertions, however it should be kept in mind that the majority of parents who completed this survey were Mothers, thus the results are likely to be more representative of mothers responses and should not necessarily be generalized to Fathers.

Contrary to expectations parents did not encourage females to ruminate in response to school, family, or social stressors more than males. Parental encouragement of rumination was more dependent on the context of the stressor than the gender of the child. Regardless of the age or gender of their child, parents encouraged rumination most frequently in response to school stressors, followed by family, and social stressors least. Such contextually-based endorsement of rumination was unexpected.

How might this finding be explained? People tend to ruminate because they believe thinking about their problems and understanding why they feel the way they do will enable them to find solutions to their current feelings of distress. That is, ruminators believe that rumination is an effective coping mechanism to relieve maladjustment (Lyubomirsky \& Tkach, 2004; Nolen-Hoeksema et al., 1999; 
Strauss et al., 1997). Thus, one possibility is that parents may encourage their children to ruminate because they perceive it to be an effective coping strategy in particular domains. However, a closer look at these data suggests that parents do not believe that rumination is an effective strategy to cope with stressful or distressing situations. In particular, the mean values for parental encouragement of rumination indicated that parents actually discouraged, rather than encouraged their children to ruminate. In all three domains (and on total scores) parents' scores fell in the range of 'strongly discourage' to 'somewhat discourage'. Thus, although in the adult literature (e.g. Lyubomirsky \& Tkach; Strauss et al.) individuals report they ruminate to solve their problems, preadolescents seemingly are not being encouraged by parents to solve their problems by thinking about their feelings. In essence, preadolescents are being discouraged to ruminate, and this is true for males and females, regardless of their age. However, such discouragement is potentially unlikely to reduce the development of a ruminative response style because there was no significant relationship between rumination levels of preadolescents and parental endorsement of rumination.

However a limitation of the methodology potentially created a floor effect in the parental data. Because the items in the RSQ measure a maladaptive style of coping — rumination - the items that were created to assess parental encouragement of rumination were likely to appear pejorative to parents. The pejorative nature of the items may have been exaggerated by the presentation of alternative adaptive coping responses — problem solving — thus enhancing the likelihood that parents would discourage rumination. The possibility that this 
produced a floor effect in these data, making the identification of gender differences difficult even in the presence of a true effect should not be discounted.

Regarding preadolescents perceptions of their peers, similarly to the parental results, female protagonists were not expected to ruminate more than male protagonists. Thus, contrary to predictions, preadolescents in this study did not exhibit rigid gender role stereotypes as seen in previous research (Broderick \& Korteland, 2002; Lobel et al., 2004). One possible explanation for this result involves the age of our sample. The participants in this study (9-13 years) were younger than those in Lobel et al.'s study (12-25 years). Notably, the participants in Broderick and Korteland's (2002) study were also slightly older (11-14 years) than those in this sample. According to the gender intensification hypothesis, gender becomes particularly salient during adolescence, not preadolescence (Hill \& Lynch, 1983), in part, because adolescence is when secondary sex characteristics develop (Huston \& Alvarez, 1990; Wichstrøm, 1999). This heightened salience of gender during adolescence is thought to prompt greater inflexibility of gender stereotypes and greater discriminatory behaviours when gender norms are broken (Lobel et al.).

Recent research suggests that rigid gender-based stereotypes fluctuate across development (Martin \& Ruble, 2004). For example, although younger children (5-7 years) display rigid gender expectations, older children (8-10 years) begin to display more flexible gender stereotypes (Trautner, Ruble, Cyphers, Kirsten, Behrendt, \& Hartemann, 2005). Therefore, the sample in the current study may be at a peak of gender role flexibility. Based on the gender intensification hypothesis, it could be predicted that if young adolescents (13-15 years) had been 
sampled also, results would have been congruent with the Broderick and Korteland (2002) and Lobel et al. (2004) findings. Possibly, it is not until the onset of gender intensification that certain coping strategies are considered more appropriate for males and females. However recent research does not support this view. For example, Weir and Jose (2006b) measured 287 preadolescents (11-12 years old) and 384 young adolescents (13-15 years old) using identical measures to the current study, and found that neither preadolescents or young adolescents expected female protagonists to be more ruminative than male protagonists.

It is more likely that the methodology employed in the current study accounts for the discrepancy with previous research. Research examining stereotypical expectations of behaviour often makes the stereotype (gender) salient. For example, in the Broderick and Korteland (2002) study, participants were asked to indicate how they thought most girls (or boys) would cope with the stressful situations presented in the vignette. Similarly, Strauss et al. (1997) asked participants to indicate how they thought the average male or female would respond to the situation. In contrast, in the current study, preadolescents were instructed to indicate "how they thought preadolescents would respond", based on the children that they knew, they were not asked to think about the average male or female. There are two important aspects of this instruction. First, the target was not generalized as, for example 'an average female preadolescent'; instead, preadolescents were asked to respond based on real targets- "children you know". Second, gender was not explicitly identified in this study; instead gender was inferred by a gender-specific picture and name. These two aspects of the current study were employed because research has shown that stereotypes become 
exaggerated when the target is generalized or when the stereotype being studied is made overly salient (Wood, Conway, Pushkar, \& Dugas, 2005). Thus, this study did not evoke exaggerated stereotypes by explicitly highlighting gender, as has been done in previous research. Instead, this study shows that when participants (both adults and preadolescents) are tested for gendered expectations without this objective highlighted, there are no expected differences between males and females regarding ruminative tendencies. In other words, neither parents nor preadolescents expect specific females to ruminate more than specific males.

Another interesting aspect of the results in this study is that peers (rather than parents) were highlighted as potentially important influences in the development of a ruminative response style. Specifically, preadolescents (both male and female) were likely to ruminate if they expected other preadolescents to ruminate, and vice versa. This may simply represent an egocentric bias, where preadolescents perceive others as being similar to them. Alternatively, it may be that peers play a role in the development of response styles. Research investigating the influence of peers in the onset of depressive symptoms has shown that associating with depressed peers predicts increases in depressive symptoms longitudinally, even after controlling for initial symptoms (Stevens \& Prinstein, 2005). This ‘peer contagion’ perspective of depression may also apply to responses to depression such as rumination. In other words, preadolescents may learn to ruminate from their peers. In support of such an assertion, Rose (2002) has found that adolescents who ruminate about their problems are also more likely to coruminate with their friends. By engaging in co-rumination, preadolescents are modeling ruminative behaviour and encouraging rumination in others. This area of 
research is relatively new, and thus the effects of co-rumination on intrapsychic rumination are not well understood. However, the finding that preadolescent rumination is related to expectations that other preadolescents ruminate suggests that peers may be an important area for future research interested in examining how response styles develop and become stabilized.

In sum, this chapter aimed to provide an initial investigation into how parental and peer influences correspond with actual rumination rates in preadolescents. Results from this preliminary study suggest that parental influences in the preadolescent period are not strong, and that, in contrast, peer influences may play a role in developing a response style strategy. Furthermore, preadolescents do not appear to expect others to respond to distress in genderspecific ways. Instead, preadolescents expect other preadolescents to respond in similar ways to themselves. 


\section{Chapter 4: The Development of a Scale to Measure Perceptions of False Self for Adolescents: Reliability, Validity, and Longitudinal Relationships with Maladjustment}

Study 1 (chapter 2) showed that when rumination was compared with other

known risk factors for depressive symptoms, its effect on depressive symptoms was

small, and indirect. The current chapter introduces an additional risk factor of maladjustment during adolescence, a particular type of self discrepancy: namely, perception of false self. False self perception will be compared to rumination (and sense of control) in its ability to predict maladjustment (depressive and anxious symptoms) in the following chapter. The current chapter reviews the research on false self and maladjustment during adolescence, and describes the development of a new measure named the Perception of False Self Scale (POFS). A new false self scale was constructed because the current measure-the Say What I Think Around Others Scale (SWIT; Harter \& Waters, 1991)-is confusing to adolescents and has not been subjected to stringent tests of its psychometric properties. Furthermore, the SWIT scale measures false self in specific social contexts, rather than being a measure of generalised false self perception.

The relationship between emotional disturbance and self discrepancy (e.g., actual vs. ideal selves/real self vs. false self) has a long history in psychology (Baumeister 1987; Higgins, 1987; James, 1890; Winnicott, 1965). Self discrepancies may be particularly prevalent during adolescence because even though self-concept becomes increasingly diversified during this time (Harter \& Monsour, 1992), adolescents may lack the cognitive skills required to incorporate this diversification into a unified, abstract self-concept (Fischer, 1980). Across a series of studies Harter an colleagues have noticed that contradictions in self concept, for 
example "I am happy with my friends, but unhappy with my family", prompt concern from some adolescents as to which aspect represents their "true self" and which represents their "false self" (Harter et al., 1996). False self behaviour lacks a sense of authenticity and is coupled with the phenomenological experience of being "phoney" (Harter, 2002). Specifically, false self behaviour is defined as "the extent to which one is acting in ways that do not reflect one's true self as a person, or the 'real me'” (Harter et al., p. 360). Not all contradictions in self-concept are experienced as false self behaviour however. Some adolescents feel that acting differently across different situations is part of their true self. This latter experience represents a relational self-concept, where adolescents experience 'multiple selves' across different situations (Harter, Bresnick, Bouchey, \& Whitesell, 1997). The critical difference between the concept of multiple selves and the false self concept is that the former is a normal process of development, whereas the latter is experienced as inauthentic (Harter, 1999).

One such correlate of false self behaviour is perceived conditional support from parents and friends. Harter et al. (1996) found that adolescents engaged in more false self behaviour if support from parents and classmates was perceived as conditional. This study also showed that false self behaviour was associated with heightened depressed and anxious mood, especially when adolescents perceived their true self as negative or wanted to hide who they 'really were' from others. In later studies it has become evident that false self behaviour is closely related to being unable to express one's opinions (Harter, Waters, \& Whitesell, 1997). For example, when adolescents were asked to describe what they thought false self behaviour consisted of, they reported not being able to voice one's opinions or say 
what they meant (Harter, Waters et al.). Because adolescent's descriptions of false self behaviour related largely to an inability to express one's opinions, false self behaviour has recently been conceptualized as a lack of voice (Harter, 2002). As with more general conceptualizations of false self behaviour, a lack of voice is associated with a range of negative outcomes, such as low self-esteem and higher depressive affect (Harter, 1999). Although such assertions are common in theoretical accounts of lack of voice (e.g., Gilligan, 1993, Jack, 1991), Harter, Bresnick et al. (1997) were the first to empirically verify this hypothesis in an adolescent sample.

The conceptualization of false self behaviour as 'lack of voice' is similar to the self-silencing hypothesis described in the adult literature (Jack, 1991; Jack \& Dill, 1992). Jack has asserted that women silence their thoughts, feelings, and behaviours in an attempt to maintain harmony within romantic relationships, and are consequently more likely to experience depressed mood than males. Thus, hiding one's true thoughts, feelings, and desires results in the self of a female not being nurtured, which prompts depression (Jack, Jack \& Dill). Various studies have found that self-silencing behaviour is associated with depressive symptoms (Besser, Flett, \& Davis, 2003; Cramer, Gallant, \& Langlois, 2005; Cramer \& Thoms, 2003; Duarte \& Thompson, 1999; Page, Stevens, \& Galvin. 1996). However, the claim that females self-silence more than males has not been empirically supported. In fact, males appear to self-silence at either the same rate or more frequently than females (Cramer \& Thoms; Duarte \& Thompson; Page et al.). Similarly it appears that false self behaviour is not more common in female adolescents' (Harter, 1999). 
Self-silencing behaviour and false self behaviour are theoretically similar in at least three ways. First, both assert that in an attempt to hide one's true thoughts and feelings, people present an image that is inconsistent with their true selfconcept, which is then experienced as phoney and false. Second, both are thought to be more prominent in relationships where affection or approval is perceived as conditional. And third, both involve the hypothesis that hiding one's true thoughts and feelings results in maladjustment. The conceptual similarities between selfsilencing and false self behaviour suggest that some aspects of self-silencing may be appropriately used to assess false self in adolescents. Empirically, these two scales correlate in college students (Smolak \& Munsterieger, 2002), but similarities and differences between these two scales have not been assessed in adolescent samples.

The measurement of false self behaviour in adolescents was originally assessed through the multiple self procedure (Harter, Bresnick et al., 1997), where adolescents describe attributes (both similar and contradictory) they display with significant others (mother vs. father). In this procedure, false self behaviour is measured by the number of conflicting attributes reported. Adaptations of this procedure have also been used with adults (Neff \& Harter, 2002). However, the multiple self procedure is less optimal than a survey measure because it is time consuming and requires the researcher to work individually with each participant. Questionnaires assessing false self, such as the Say What I Think Around Others scale (SWIT; Harter \& Waters, 1991), have also been used successfully in adolescent samples (Harter et al., 1996), and responses to questionnaires appear to converge with responses on the multiple self procedure (Harter, Waters et al., 1997). However, the SWIT scale assesses only one aspect of false self behaviour 
(lack of voice) and has not been subjected to stringent psychometric assessment. In addition, the SWIT false self questionnaire follows a format, which some adolescents and young adults have found confusing. Specifically, the format is identical to Self-Perception Profile for Children (Harter, 1985) where adolescents are presented with two statements e.g. "Some people share what they are thinking with male classmates BUT Other people find it hard to share what they are really thinking with male classmates". Adolescents choose which statement best represents them, and then they indicate if the selected statement is "sort of true", or "really true" about them. In previous research adolescents and young adults are confused by this format in that they often respond to each half of the statement, rather than choosing only one statement.

Furthermore, because only one social context is assessed at a time (e.g. male classmates), in order to assess false self behaviour across multiple contexts, the measure needs to be administered multiple times, each time with a different reference group. Consequently, administration can become long and repetitive. Thus, a goal of the current study was to develop a new false self scale and rigorously test both its psychometric properties and its relationship with maladjustment. By developing a new scale, perceptions of false self can be compared with rumination to test RST against vulnerability factors other than sense of control.

To develop the false self scale, a multi-method approach was adopted. First, interviews were conducted with adolescents to explore what they perceived false self behaviour to be. Next, to develop items for the POFS scale, themes from the SWIT scale (Harter \& Waters, 1991), and from two of the subscales on the Silencing the Self Scale (STSS; Jack, 1991)-externalized self perception (ESP) and 
silencing the self (SS). The ESP measures how much an individual's behaviour is influenced by perceived external standards rather than self-generated internal standards. Themes from the ESP subscale were used because adolescents have reported endorsing false self behaviour when they felt support from parents and peers was conditional (Harter et al., 1996). Second, themes were extracted from the SS subscale, which measures the extent to which an individual avoids conflict within a relationship by silencing one's own opinion. SS themes were used because the descriptions of false self behaviour given by adolescents involved a 'lack of voice' (Harter, Waters et al, 1997; Harter 1999).

During development of the POFS scale, a major consideration was whether the items should be contextually based (e.g., placed within a specific social context) or represent general false self behaviour and perceptions. Both the multiple self procedures and questionnaires developed by Harter and colleagues assess contextually based false self behaviour. That is, they assess false self behaviour within a specific relational context, such as with teachers or close friends. They constructed their measure this way largely because adolescents have reported displaying differential levels of voice depending on the social context, for example, adolescents display more authentic voice with friends than with opposite-sex classmates (Harter Waters et al., 1997). However, adolescents report that false self behaviour is expressed similarly regardless of the social context, e.g., through "lack of voice" (Harter, Water et al., 1997). Because the contextual difference in false self behaviour appears to be quantitative rather than qualitative, a general false self scale was developed. In this study, the assumption that a generalised measure would 
relate strongly with a context specific measure of false self behaviour was tested. Specifically, I correlated responses on the POFS scale with the SWIT scale.

There were two additional goals of the current chapter. First, I aimed to assess a wider range of false self behaviours than lack of voice. Harter, Waters et al., (1997) found that a significant proportion of false self behaviour is expressed as loss of voice, thus this is certainly an important type of false self behaviour, however additional false self behaviours also exist. Thus, in the present study, interviews with adolescents were undertaken to explore other types of false self behaviour, which could be incorporated into an assessment tool.

Second, I aimed to test whether false self could predict change in maladjustment across time. The majority of research examining the relationship between false self behaviour and maladjustment has used concurrent data. Consequently, a number of empirical questions regarding false self behaviour and maladjustment across time remain untested. One such area relates to the stability of false self behaviour across time. If an adolescent perceives him or herself as behaving in a way that is false, does this self-concept remain relatively stable across time? Another understudied area relates to the direction of effect between false self and maladjustment across time. Does false self behaviour lead to increases in maladjustment across time, or does maladjustment lead to increases in false self behaviour across time? One possibility is that the relationship between false self and maladjustment is bi-directional (Harter, Waters et al., 1997), that is, false self behaviour increases maladjustment, while maladjustment at the same time increases false self behaviour. It was predicted here that the relationship between false self and maladjustment would be bi-directional. 
Overview of Studies

Three studies were conducted. In study 1 , adolescents were interviewed about their true/false self perceptions. From these interviews, language and themes for item content were extracted. Interviews also examined whether false self behaviour was entirely or predominantly expressed as loss of voice, or if alternative expressions were also meaningful to adolescents. From these interviews, and based on the theoretical and empirical literature reviewed above, a preliminary battery of items was developed. Study 2 involved two phases. Phase 1 involved piloting the initial battery of items with adolescents. An exploratory factor analysis was performed on this initial battery of items to select the best items for the final scale. Content validity of items was also considered when choosing items for elimination. Phase 2 involved a follow up administration 10 weeks later of the reduced scale. The goal of this second phase was twofold; first, to confirm the factor structure using confirmatory factor analysis and second, to explore the longitudinal association between false self behaviour and maladjustment. In study 3, responses on the POFS scale were compared with two pre-existing measures: The SWIT scale (Harter \& Waters, 1991) and the STSS (Jack, 1991). Study 3 also compared the relationships among the three false self scales and maladjustment.

\section{STUDY 1}

\section{Method}

Participants and Procedure

Participants were recruited from four schools throughout the Wellington region of New Zealand. Regarding the socio-economic status of the schools involved, deciles 2, 6, 7, and 8 were represented. In total, 24 interviews were conducted with 12 males and 12 females. Specifically, the sample was composed of 
6 eleven year olds ( 2 males, 4 females), 4 twelve year olds (all male), 5 thirteen year olds (3 males, 2 females), 4 fourteen year olds ( 1 male, 3 females), 4 fifteen year olds (2 males, 2 females), and 1 sixteen year old (female). Although ethnicity was not recorded during the interviews, the participants had similar ethnic makeups to other adolescents in New Zealand, that is, they were predominantly European New Zealanders.

Participating schools recruited students via daily notices and/or class announcements for a study advertised as the "Life Events of Adolescents Study". Interested students were given an information sheet and parental consent form. Interviews commenced after parental consent was obtained, and were conducted in a private room allocated by the school. To increase rapport, the male participants were interviewed by a male interviewer, and the female participants were interviewed by a female interviewer. A description of the study was discussed with the participants before their assent to participate was obtained. No adolescents' decided to not participate based on the studies description. All interviews were tape recorded and then later transcribed. Each interview lasted approximately 30 minutes. Participants received a movie/book voucher for their time, and the schools received $\$ 5$ in book vouchers for each participant. At the end of the interview, participants were thanked, debriefed, and any questions were answered. Explanation of False Self Behaviour to Adolescents

To reduce acquiescent bias, false self behaviour was described to adolescents as a discrepancy between public and private self perception. More specifically, the false self behaviour was described in the following way: 
"I'd like to talk to you about public and private selves; I am interested in the different parts to an adolescent's life, and the different people in adolescent's lives. Sometimes people feel like they are different in public to what they are really like in private. So, people might act differently to how they really feel. I'd like you to think of your private self as your true self, and your public self as how you act in front of others. I am interested in whether you feel there is a difference between your public and your private self or not, and how you feel about any differences or similarities that there might be".

To further describe the distinction between public and private selves, a visual depiction was also presented, created for the purpose of this study. The private self and the public self were depicted as circles which overlapped to different degrees (Figure 4.1). Three examples were shown: where the selves almost completely overlapped, where they partially overlapped, and where they were completely separate.

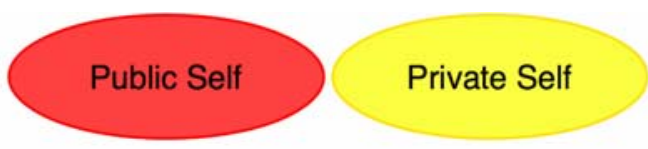

\section{Public and Private Selves are clearly distinct}

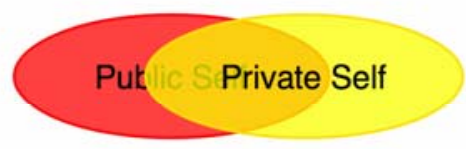

Public and Private Selves overlap partially

\section{Private Self}

\section{Public and Private Selves overlap considerably}

Figure 4.1. Graphical depiction for adolescents of complete, partial, and no discrepancy between public and private self perceptions

On a separate page, adolescents were asked to place two circles (one representing their public self and the other representing their private self) in a way that represented how much overlap they thought existed between their public and private selves. Once they had placed the circles, the interviewer discussed with the 
adolescent why they placed the circles in that fashion, and asked the adolescent to describe examples of when there were differences and similarities between their public and private selves, and how they felt about these differences and similarities.

\section{Results and Discussion}

\section{Extraction of Themes from Interviews}

Themes from the interviews were extracted using techniques drawn from thematic and content analysis (Flick, 2006). Each transcribed interview was analysed separately first. Specifically, each expression of false self behaviour described by the interviewee was categorised (e.g. displaying false emotion). In addition to the type of false self behaviour expressed, the associated experience of the behaviour was noted (e.g. embarrassed, sad). Once the individual interviews were analyzed, the descriptions and experience of false self behaviour across participants were compared. The overarching themes identified across the interviews were then compared with existing definitions and descriptions of false self behaviour to verify existing characterisations of false self behaviour or identify additional false self behaviours not currently described in the literature.

Presence of False Self Behaviour

Overall, results were similar to previous findings (e.g., Harter et al., 1996; Harter, 1999) in that false self behaviour (distinction between their private self and public self) was experienced as phoney, and in contradiction to their true selfconcept. In addition, adolescents' descriptions of false self behaviour were similar to those already identified in the literature. Specifically, the predominant expression of false self behaviour was loss of voice. However adolescents also described false self 
behaviour as presenting themselves falsely (e.g. appearance) and displaying false emotion.

Voice. When describing false self behaviour, participants spoke largely about their lack of voice. These results are similar to the theme of "lack of voice" already identified in the literature (Gilligan, 1993; Harter, Waters et al., 1997; Jack, 1991). Participants described their false self behaviour as not being able to voice their opinion or not being able to say what they really meant, "sometimes I won't tell people how I really feel, like they will ask me a question and I'll keep it quiet, I won't answer them how I want to answer it”. The reported reasons for engaging in false self behaviour, and the outcomes of false self behaviour were also similar to those found in previous research. For example, adolescents reported engaging in false self behaviour because they devalued their true self, and reported that false self behaviour resulted in maladjustment "It feels bad when I keep things from people, I can't talk to anyone, not even my mum. I wish I could but I can't".

Evidence for false self behaviour as lack of voice also stemmed from adolescents' descriptions of their true self behaviour. When asked to describe instances where there was a distinction between public and private selves, some adolescents replied that there was no distinction because they could voice their opinions, "um, most of the time I say what I think... most of the time I'm pretty up front". Thus, true self behaviour is viewed largely as saying what one thinks and being honest with others, whereas false self behaviour is viewed as not being able to say what one thinks or not expressing one's true thoughts or opinions.

Appearance/Presentation. In addition to voice, true self behaviour was expressed through adolescents' appearance and presentation. Adolescents reported that the 
way they dressed reflected true self behaviour, "the way I look, I present myself the same wherever I go, everyone knows that I don't change for anyone I am just who I am and sometimes people think I have the weirdest style in the world but that's just who I am and I don't like going along with trends". False self behaviour was described as dressing in a way to impress others, rather than having one's own style. Therefore, true and false self behaviour can also be represented in an adolescent's appearance, and well as in their ability or willingness to express their voice.

Emotion. False self behaviour also included hiding one's true feelings or masking one's true emotion. These feelings were often hidden because the feelings themselves were negative, "I used to like hard out pretend, it was like I was wearing a mask, I was completely different at home to how I was in public... I was like, always happy and when I was at home I was always crying. I'd pretend". This theme of experiencing negative internal feelings but projecting positive feelings was a common source of discomfort. Another adolescent commented, 'It doesn't make me feel right because I'd rather be myself than this big happy smiley face." Thus, false self behaviour was described as behaviour that did not express their true feelings and/or behaviours which masked true feelings.

Summary of Study 1

When asked about the distinction between their public and private selves (false self behaviour), adolescents reported some similar themes to those already identified in the literature. Namely, false self behaviour was described mainly as a loss of voice, where an adolescent felt they could not voice their true opinion or say what they really wanted to say. Two additional types of false self behaviour were identified in the interviews. First, false self behaviour was described as presenting 
oneself as different to how they thought they truly were, e.g., through the clothes they wore. Second, false self behaviour was described as hiding one's true emotions or pretending to be happy when one felt sad. Importantly, the descriptions of false self behaviour replicated previous findings (Harter et al., 1996; Harter, Waters et al., 1997).

\section{STUDY 2: PHASE 1}

Phase 1 involved generating a battery of potential items for the new false self scale and pilot testing these items in a sample of adolescents. Because the scale was not a specific assessment of behaviours, but rather was a generalised self concept measure, the scale was named the Perceptions of False Self (POFS). A series of exploratory factor analyses, along with consideration of the reliability and validity of the scale enabled this pilot version to be reduced to a shorter version.

\section{Method}

Item Development and Scale Design

As described above, items were developed based on interviews with adolescents and the existing literature on false self and self silencing. Initially, a pool of 63 items was developed. Twenty four items tapped issues of voice (Harter 2002; Jack 1991), e.g., "I hide what I really think if it is different to others", and 10 of these were reverse-worded. Eighteen items measured false presentation, e.g. "I change my appearance if I think others do not like it”, 5 of which were reverseworded. Twelve items assessed false emotion, e.g. "even if I am sad or angry on the inside, I try to look happy on the outside”, 3 of which were reverse-worded. And last, 9 items measured externalised self-perception (Jack), "I judge myself by how I think other people see me”, 2 of which were reverse-worded. From this initial pool, 
20 items were deleted from the scale due to lack of clarity, readability, or item redundancy, leaving an initial item pool of 43 items.

All items were developed as closed-ended statements and scored using a 5point Likert scale $(1=$ strongly disagree, $2=$ disagree, $3=$ neutral, $4=$ agree, $5=$ strongly agree). The instructions for the scale were as follows:

"Below is a list of ways people may see themselves, and I am interested in whether you see yourself in any of these ways. You may agree or disagree with these statements about you. Please circle the number that best describes how you see yourself. There are no right or wrong answers, I am interested in how you really see yourself, NOT how you think you should see yourself."

Participants and Procedure

Forty one schools throughout the Wellington region were sent a letter informing them of the study and given an invitation to participate. These schools were then called for an expression of interest in participation; six schools across the Wellington region were recruited ${ }^{22}$. Regarding socio-economic status, one school was decile 5, two schools were decile 6 , one school was decile 7 , one school was decile 9 , and the remaining school was decile 10 . Two hundred and sixty-seven adolescents (79 males, 188 females) participated in the pilot. Participants were aged between $11-15$ years $(M=12.99, S D=1.33)$. The majority of participants were Pakeha/European New Zealander (63\%), 13\% were Maori, 5\% were Pacific Islanders, 5\% were Asian, and 14\% classified their ethnicity as "other". Participants completed the 43-item POFS scale ${ }^{23}$ along with measures of anxious and depressive symptoms. Participants completed the battery of measures in groups, ranging in size between 5 and 40 adolescents. Each scale was explained to the participants prior to commencement. Parental consent was granted prior to data

\footnotetext{
${ }^{22}$ A total of 876 consent forms were provided to these six schools

${ }^{23}$ See Appendix G for the POFS scale
} 
collection, and the participants also gave their assent to participate after hearing further information about the study.

Measures

Revised Children's Manifest Anxiety Scale ${ }^{24}$. The Revised Children's Manifest Anxiety Scale (RCMA; Reynolds \& Richmond, 1997) is a 28-item $($ yes $=1$, no $=0$ ) measure of anxious symptoms. Scores range from 0-28; anxious symptoms are typically indicated by a score of $1 S D$ above the mean in a given age range (Reynolds \& Richmond). The RCMA has good internal reliability (Jolly, Aruffo, Wherry, \& Livingston, 1993), and in this study, the scale also exhibited excellent internal reliability $(\alpha=.86)$.

Children's Depression Inventory. Adolescents also completed the Children's Depression Inventory (Kovacs, 1992), previously described in Chapter 2. In this sample, the CDI exhibited excellent internal reliability $(\alpha=.92)$. In this study, anxious and depressive symptoms were highly correlated, $r(193)=.81, p<.001$. Because this correlation was high enough to suggest the presence of significant multicollinearity (Tabachnick \& Fidell, 2001), anxiety and depression were linearly combined to represent maladjustment.

\section{Results and Discussion}

\section{Exploratory Factor Analysis}

An exploratory factor analysis (using principal components analysis) was conducted to identify items for the final scale, and explore the scale's factor structure. The presence of factors was explored using a scree plot rather than using the "eigenvalues greater than 1" rule because scales with large initial item pools (e.g.

\footnotetext{
${ }^{24}$ The RMCA scale is presented in Appendix H
} 
$>40$ ), can yield too many eigenvalues with the latter method (Giles, 2002).

Visually, the scree plot indicated the presence of two factors; eigenvalues decreased substantially after the second factor $(10.26,3.04,1.94,1.75,1.55$, etc). Thus, a second principal components analysis was conducted with two factors extracted. Oblique rotation (Direct Oblimin) was used because the factors were expected to be correlated. The first factor explained $23.85 \%$ of the variance, and the second factor explained an additional $7.06 \%$ of the variance.

Twenty-two items loaded uniquely on the first factor $(.36-.72)$. Twelve items loaded on the second factor $(.33-.68)$. Four items loaded on both factors, and 5 items did not load on either factor. Items which either double-loaded or loaded below .30 on either factor were omitted from the scale. Items on the first factor were seen to tap the concept of false vs. true self ('I don't let people see the real me"; "I act in ways that express who I really am"), and this factor was named “false self". False self items included general false self behaviour (e.g. "I hide the 'real me') and specific false self behaviours such as loss of voice, presentation, and emotion. Items loading uniquely on the second factor captured high social concern ("I spend a lot of time thinking about how other people see me"), and was named "social concern".

Consequently, 34 items remained for possible inclusion in the final scale. Because a short scale (approximately 20 items) was desired, further items were deleted based on three criteria: a) items which did not exhibit high face validity, b) items with ambiguous language, overlapping item content, or low readability, and c) items which exhibited inadequate correlations among individual items (as indicated by MSA/individual KMO scores). First, 6 items were deleted because of 
low face validity. Second, 4 items were deleted because of overlapping item

content, and 2 items were deleted for ambiguous language. And third, 6 items were

deleted because their individual measures of sampling adequacy were below .60

(Pett, Lackey, \& Sullivan, 2003). These decisions left 16 items in the final version of

the POFS scale, three of which were reverse-scored.

To test the refined POFS scale, a subsequent exploratory factor analysis

was conducted with two factors extracted, using Direct Oblimin rotation (factor

loadings are presented in Table 4.1). Eleven items loaded on the 'false self' factor,

and explained $30.04 \%$ of the variance. This first factor exhibited high internal

reliability $(\alpha=.83)$. Five items loaded on the 'social concern' factor, and explained

a further $9.15 \%$ of the variance. In total, $39.16 \%$ of the variance was explained by

the 2 -factor model. These two factors were positively correlated $r(265)=.47, p<$

.001 .

Table 4.1. Factor Loadings for the 1-Factor and 2-Factor Models of the POFS Scale

\begin{tabular}{|c|c|c|c|}
\hline & \multirow[t]{2}{*}{ 1-Factor } & \multicolumn{2}{|c|}{ 2-Factor } \\
\hline & & FS & SC \\
\hline I say what I think even if it is different to the opinion of others* & .46 & .31 & .26 \\
\hline I cannot express my opinion & .49 & .37 & .22 \\
\hline I act one way, but want to act a different way & .60 & .59 & -.06 \\
\hline I don't let people see the real me & .70 & .77 & -.07 \\
\hline My thoughts are not important to others & .49 & .59 & -.13 \\
\hline I hide the real me by looking like others & .69 & .70 & .02 \\
\hline I act in ways that express who I really am* & .58 & .65 & -.12 \\
\hline I hide my true feelings if I think they will upset others & .43 & .07 & .59 \\
\hline What I say on the outside is different to what I think on the inside & .69 & .70 & .04 \\
\hline I tend to say one thing even when I think another & .58 & .55 & .08 \\
\hline $\begin{array}{l}\text { If people knew what I was really like on the inside they would not } \\
\text { like me }\end{array}$ & .72 & .70 & .07 \\
\hline I can talk openly to others about my feelings* & .56 & .56 & .04 \\
\hline I stay quiet when I don't agree with others & .46 & .15 & .53 \\
\hline I don't like to look different from other people & .46 & .26 & .34 \\
\hline I spend a lot of time thinking about how other people feel & .31 & -.19 & .82 \\
\hline Other people's feelings are more important than mine & .36 & -.02 & .63 \\
\hline
\end{tabular}


However, the social concern factor exhibited low internal reliability $(\alpha=$ .60), and no items, if deleted, would have improved the internal reliability of the scale. A further problem was that an examination of the scree plot suggested that the modified scale may now be best explained by a 1 -factor model; eigenvalues decreased substantially after the first factor $(4.81,1.46,1.09,1.03)$. To test the 1 -factor model, a final EFA was performed extracting 1 factor (Table 4.1). All items loaded above .30 on the 1 -factor model, and the scale exhibited high internal reliability $(\alpha=.84)$. Due to this increase in internal reliability, the 1-factor model was considered the best representation of these data.

Criterion Validity

POFS scores were correlated with maladjustment (anxious and depressive symptoms linearly combined) to explore criterion validity. As expected, POFS scores were associated with higher maladjustment, $r(265)=.65, p<.001$, supporting previous research with false self behaviour and maladjustment (Harter et al., 1996). Summary of Phase 1

A measure of generalised false self perception was developed. The results of the exploratory factor analysis demonstrated that the 16-item POFS scale conforms to a 1-factor model. This 1-factor model incorporated different aspects of false self behaviour such as loss of voice, appearance, and emotion, while also assessing more abstract self perceptions of false self behaviour. This new scale showed excellent reliability and validity (both face and criterion). Similarly to previous research (Harter et al., 1996), reported false self behaviour was related to increased levels of maladjustment. 


\section{STUDY 2: PHASE 2}

Ten weeks after the pilot, a second data collection was conducted using the 16-item POFS scale. Five goals were posed for this follow-up: a) to explore age and gender effects in perceptions of false self, b) to rigorously test the established 1-factor model using confirmatory factor analysis, c) to test the stability of POFS scores across time, d) to further test the validity of the scale, and e) to explore the longitudinal relationships between false self and maladjustment.

\section{Method}

\section{Participants and Procedure}

Two hundred and thirty five (76 males, 159 females) adolescents participated in the second data collection. The age of these participants ranged between 11 and 16 years olds $(M=12.98, S D=1.39)$. This sample of 235 adolescents was used for the confirmatory factor analysis only. Of these 235 adolescents, 195 (68 males, 127 females) had participated during the first phase of data collection. New participants ( $\mathrm{N}$ $=40$ ) had either returned a consent form but were absent from class on the day of the pilot, or had returned a consent form in between the pilot and follow up. New participants were included in the study to allow for a slightly varied sample for the confirmatory factor analysis. The responses of the 195 adolescents who participated at both time points were involved the longitudinal analyses. Ethnicity of participants did not change substantially in the final sample. Attrition at 10 weeks was predominantly due to absence at follow up, however 13 participants were deleted from the sample because they completed less than $95 \%$ of one or more survey in the questionnaire. The procedure was identical to that described in phase 1 , and the same anxiety (RMCA) and depression (CDI) scales were used. Both the RMCA and the CDI 
continued to exhibit excellent internal reliability (.90 and .92 respectively), as did the 16-item POFS scale (.88). Because RMCA and CDI scores were still highly correlated at follow up, $r(193)=.79, p<.001$, the variables were again linearly combined to represent a maladjustment score.

\section{Results and Discussion}

Age and Gender Effects on the POFS Scale

To explore the effects of age and gender on false self perceptions, participants were grouped as either younger than 13 years old $(\mathrm{N}=94)$ or 13 years and older $(\mathrm{N}$ $=101)$. This grouping was performed to compare preadolescents with adolescents. A 2 (age: $<13$ yrs, $\geq 13$ yrs) x 2 (gender: male, female) repeated measures ANOVA was conducted on POFS scores. Tests of within-subject effects indicated that participants reported marginally different levels of false self, $F(1,191)=3.21, p=$ .08 , suggesting that one's perception of false self behaviour remains relatively stable across short periods of time. Furthermore, test-retest reliability between POFS scores across the 10 weeks was very high $r(193)=.84, p<.001$, verifying further that perceptions of false self are relatively stable across short periods of time. None of the interactions, time $\mathrm{x}$ gender, $F(1,191)=.98, p=.32 ;$ time $\mathrm{x}$ age, $F(1,191)=.15, p$ $=.70$; nor time $\mathrm{x}$ gender $\mathrm{x}$ age, $F(1,191)=.37, p=.54$, reached significance.

Between-subjects results demonstrated that males and females reported similar levels of false self perceptions, $F(1,191)=.02, p=.90$, replicating previous research. Likewise, preadolescents $(<13$ yrs) and early adolescents $(\geq 13 \mathrm{yrs})$ reported similar levels of false self perceptions, $F(1,191)=.46, p=.50$. Means and standard deviations for males and females for both age groups on the POFS scale are presented in Table 4.2. The age by gender interaction was not significant, $F(1,191)=$ 
$.43, p=.51$. In summary, males and females across different ages reported similar levels of false self perceptions, and these false self perceptions remained stable across the ten week period.

Table 4.2. POFS Means and Standard Deviations for Males and Females at Both Time Points

\begin{tabular}{lcccccc}
\hline & \multicolumn{2}{c}{ Males } & \multicolumn{2}{c}{ Females } & \multicolumn{2}{c}{ Total } \\
\hline & $M$ & S.D. & M & S.D. & M & S.D. \\
\hline & \multicolumn{5}{c}{ Time 1} \\
$<13$ years & 40.97 & 10.62 & 39.41 & 10.88 & 40.04 & 10.75 \\
$\geq 13$ years & 39.43 & 7.30 & 39.48 & 10.73 & 39.17 & 9.81 \\
\hline \multirow{2}{*}{$<$ 13 years } & 39.21 & 12.58 & 39.14 & 11.37 & 39.17 & 11.81 \\
$\geq 13$ years & 37.60 & 9.50 & 39.00 & 10.67 & 38.58 & 10.31 \\
\hline
\end{tabular}

Confirmatory Factor Analysis

A confirmatory factor analysis was conducted on the 235 individuals who participated at follow-up to confirm the 1 -factor model found using Time 1 data. CFA was employed because it can rigorously test an a posteriori factor structure using various fit indices (Bryant \& Yarnold, 1995). To determine whether the 1-factor model fit these data well, the fit indices described in Chapter 2 were used.

Specifically, the $\chi^{2} / d f$ ratio, the GFI, the AGFI, the sRMR, and the RMSEA were assessed. All 16 items were stipulated to load onto the single latent variable False Self. As can be seen in Figure 4.2, all items loaded significantly on the latent variable (.34 -

.77). However, overall fit indices fell below acceptable levels, $\chi^{2} / d f=3.07$; RMSEA $=.09 ; \mathrm{sRMR}=.07 ; \mathrm{GFI}=.85 ; \mathrm{AGFI}=.80$. 


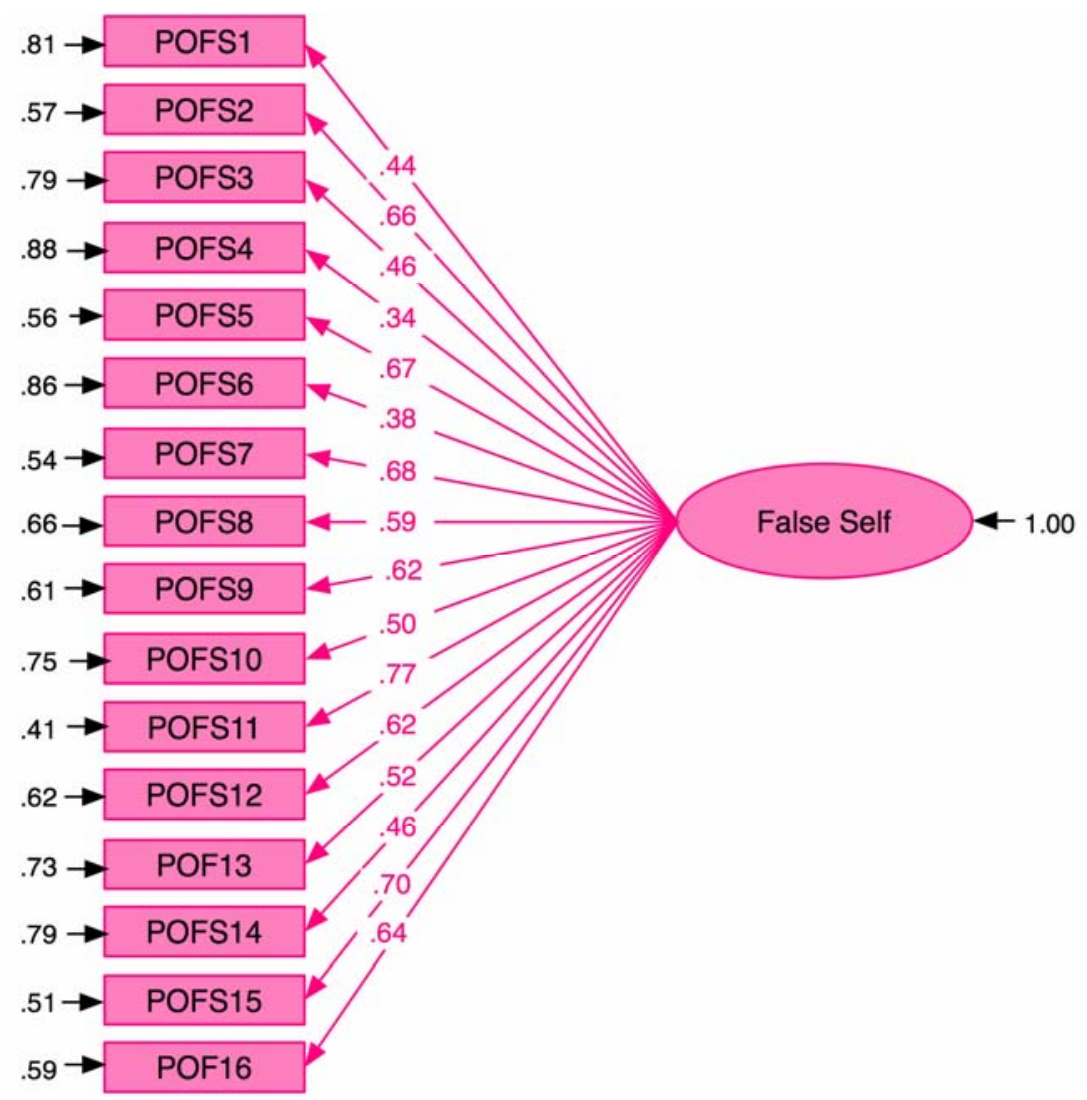

Figure 4.2. Confirmatory factor analysis of the POFS scale with 1-factor extracted

In an attempt to improve the model fit indices, the 1 -factor model was reanalyzed using 8 parcels of items ${ }^{25}$. Parcels were employed because previous research has found that parceled items can improve model fit indices in small samples (Little, Cunningham, Shahar \& Widaman, 2002). An important assumption of parceling is that the items are unidimensional, that is, the items do not measure multidimensional aspects of a construct (Bandalos \& Finney, 2001). In the case of the POFS scale here, the EFA presented earlier indicated that all items tapped one underlying dimension, thus meeting the assumption of unidimensionality. When the 8 parcels (indicators) were loaded on the latent variable of false self, the strength of the

\footnotetext{
${ }^{25}$ Parcel 1included items 1-2, parcel 2 included items 3-4, parcel 3 included items 5-6 parcel 4 included items 7-8, parcel 5 included items 9-10, parcel 6 included items 11-12, parcel 7 included items 13-14, and parcel 8 included items 15-16. Parcels exhibited high internal reliability at both baseline $(\alpha=.88)$.
} 
indicators improved, ranging between .51 and .83. Furthermore, the model fit indices improved to meet acceptable standards, $\chi^{2} / d f=2.80$ RMSEA $=.08 ;$ sRMR $=.04$; GFI $=.94 ;$ AGFI $=.90$. Thus, the confirmatory factor analysis verified the 1 -factor model established previously with the exploratory factor analysis, and consequently, the POFS scale exhibits factorial validity.

Criterion Validity

Correlations were calculated to explore if baseline POFS scores were related to maladjustment 10 weeks later. As predicted, higher perceptions of false self were related to greater maladjustment across time, $r(193)=.62, p<.001$.

Longitudinal Associations Between False Self Perceptions and Maladjustment

Longitudinal relationships between maladjustment and false self perceptions were also assessed. Specifically, I tested whether initial POFS scores could predict later maladjustment while initial levels of maladjustment were controlled. In addition, it was determined whether initial levels of maladjustment could predict later POFS scores while initial levels of POFS score were controlled. In short, the possibility of a bi-directional relationship was tested. To test this possibility, a path model was constructed using structural equation modeling. The model included the autocorrelations (stability coefficients) for POFS and maladjustment, and the two cross-lag paths, one from baseline POFS to 10 week maladjustment and, and a second from baseline maladjustment to 10 week POFS. Error terms between POFS and anxiety/depression were allowed to correlate concurrently, at both time points. Resultantly, the initial model was fully saturated, thus any non-significant paths were removed to obtain fit indices (Kline, 2005). Path models were not constructed 
separately for age or gender because ANOVA results found no age or gender effects. Thus, the path model results are reported using the whole sample.

Regarding the initial model assessing the longitudinal relationship between perceptions of false self and maladjustment, the stability coefficients for both false self perceptions $(\beta=.67, p<.001)$ and maladjustment $(\beta=.82, p<.001)$ were high across the 10 week period. Perceptions of false self did not predict later depression while initial levels of depression were controlled $(\beta=.06, \mathrm{p}=.21)$. Instead, initial levels of depression predicted perceptions of false self while controlling for initial levels of false self perception $(\beta=.24, \mathrm{p}<.001)$.

Next, to obtain fit indices the model was rerun, without the path from false self perceptions to maladjustment. The reduced model (Figure 4.3) fit these data well, $\chi 2 / \mathrm{df}=1.59 ; \mathrm{RMSEA}=.06 ; \mathrm{sRMR}=.01 ; \mathrm{GFI}=1.00 ; \mathrm{AGFI}=.96$. For ease of interpretation, standardized coefficients are presented in Figure 4.3. These results show that maladjustment predicted an increase in false self perceptions across time; however, false self perceptions did not predict increases in maladjustment across time. Thus, contrary to predictions, the relationship appears to be unidirectional, rather than bi-directional.

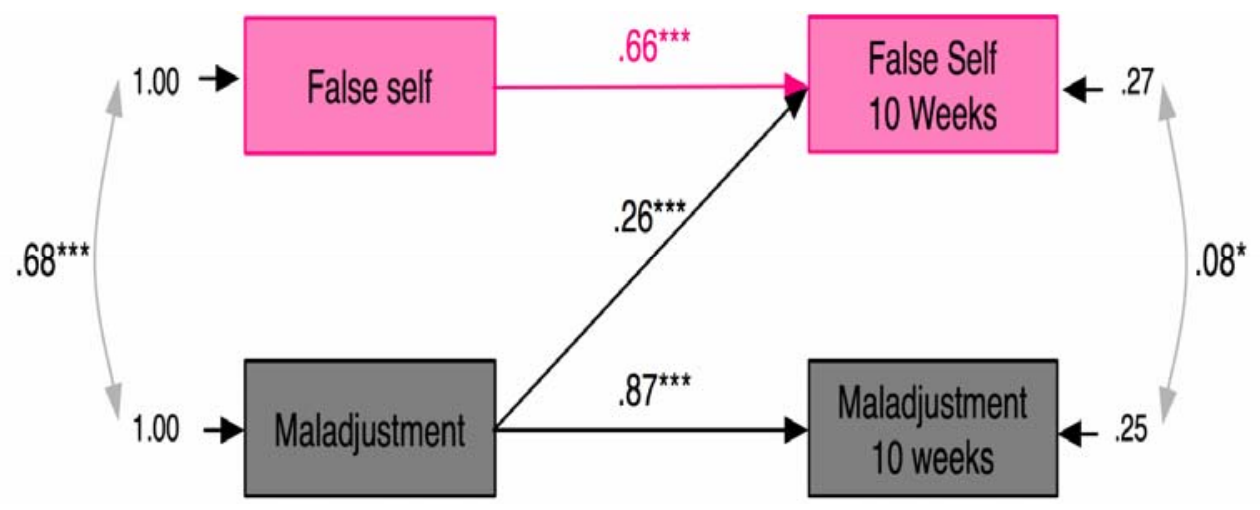

Figure 4.3. Cross lag path model between perception of false self and maladjustment across 10 weeks. $*_{p}<.05, * * * p<.001$. 
Summary of Phase 2

Males and females reported similar levels of false self perceptions, and preadolescents and early adolescents did not differ in mean levels of false self perceptions. The psychometric evidence for the POFS scale seems to be strong: under a stringent test of its factor structure (confirmatory factor analysis), the POFS scale conformed to a 1 -factor model structure, the internal reliability was replicated to be high, and the stability of false self perceptions was reasonably high across the 10 week period. The longitudinal results suggest that the relationship between false self and maladjustment may be unidirectional; maladjustment seemed to predict increases in an individual's perception of false self across time.

\section{STUDY 3}

Study 3 was conducted to test the convergent validity of the POFS scale. Specifically, a subset of participants from study 2 (this Chapter) were recruited to complete the POFS scale, the SWIT scales (Harter \& Waters, 1991) and the STSS (Jack, 1991). POFS scores were expected to be significantly correlated with both measures, and thus exhibit convergent validity. An additional goal of this study was to compare the POFS, SWIT, and STSS scales in predicting maladjustment, so depressive symptoms were also examined.

\section{Method}

Participants and Procedure

Letters were sent to the parents of 183 participants who took part in study 2. These letters invited adolescents to participate in a follow up of the "Life Events of Adolescents Study". The follow up was internet-based and occurred 14 months after the initial baseline assessment. A paper version of the questionnaire was supplied to 
participants who wanted to take part but did not have access to the internet. Fortysix adolescents took part in the follow up survey (20 males, 26 females). Participants ranged in age between 11 and 15 years old $(M=13.00, S D=1.33)$. Measures

The participants completed the 16-item POFS scale described above. They also completed the 10-item CDI short form, which correlates highly with the 27 item version described above (Kovacs, 1992). Both the POFS scale $(\alpha=.86)$ and the $\mathrm{CDI}(\alpha=.85)$ exhibited acceptable internal reliability.

Say What I Think Scale ${ }^{26}$. Adolescents completed the Say What I think Scale (SWIT; Harter \& Waters, 1991) which measures false self behaviour, characterised as a loss of voice. In this study, adolescents were asked to indicate whether or not they could say what they thought around female classmates, male classmates, close friends, and parents. The SWIT scale is structured so that for each relationship domain, there are 5 questions, each with two statements (e.g. some people share what they are thinking with female classmates BUT other people find it hard to share what they are really thinking with female classmates). Adolescents are asked to select which statement best describes them, and then indicate if the chosen statement is sort of true about them or really true about them. Higher scores indicate greater authenticity of voice in that given domain. In this sample, the internal reliability was below acceptable levels for female classmates $(\alpha=.65)$, but acceptable for male classmates, $(\alpha=.80)$, close friends $(\alpha=.76)$, and parents $(\alpha$ $=.81)$

\footnotetext{
${ }^{26}$ The Say What I think Around Others Scale is presented in Appendix I
} 
Silencing the Self Scale ${ }^{27}$. Adolescents completed the Self Silencing (SS) and the Externalised Self Perception (ESP) subscales of the STSS (Jack, 1991). The SS subscale consists of 9 items that measure the extent to which an individual avoids conflict within a relationship by silencing one's own opinion. The ESP scale consists of 6 items that measure how much an individual's behaviour is influenced by perceived external standards. In its original form participants respond with intimate (i.e. romantic) relationships in mind. However, in this study the focal relationship was made generic, i.e. referring to "other people" instead of romantic relationships. In this sample, the SS subscale exhibited acceptable internal reliability $(\alpha=.78)$, however the ESP subscale did not $(\alpha=.60)$.

\section{Results and Discussion}

One reason stated above for developing the POFS scale was that in previous research, adolescents and young adults have had difficulty with the format of the SWIT scale. Harter (1985) derived this format to reduce response bias. Harter asserts that listing two opposing statements implies that half of adolescents relate to one of the statements, thus reducing socially desirable responses. However, often people are confused by this format, indicated by making a response to both statements in one question. Even in this small follow up study, this type of error was made 14 times on the female classmates subscale, 10 times on the male classmates, 2 times on the close friends subscale, and 5 times on the parents subscale. Notably, these errors were made even though for each question it was specifically stated to select only one statement.

\footnotetext{
${ }^{27}$ The Silencing the Self Scale used in this study is presented in Appendix J
} 
The primary goal of the current study was to test the convergent validity of the POFS scale. Table 4.3 shows the interrelationships among the three self perception measures. The POFS scale correlated significantly with the SWIT, SS and the ESP subscales, indicating that the POFS scale exhibits convergent validity. Table 4.3 also shows the correlations among each self-perception measure and depressive symptoms. Each self perception scale was related to depressive symptoms in the expected direction; however the POFS scale appears to have correlated to the strongest degree. In addition, the test-retest reliability for POFS scores $r(44)=.67, p<.001$, and CDI scores $r(44)=.58, p<.001$, were found to be moderately high across the 14 month period. In summary, the format of the POFS scale was easier for adolescents to complete, and the new scale exhibited higher criterion validity than existing measures of false self behaviour. Furthermore, it appears that perceptions of false self are a stable trait-like construct, which remains relatively stable across 14 months.

Table 4.3. Correlations Among Self Perception Scores and Depressive Symptoms.

\begin{tabular}{lccccccc}
\hline & POFS & SWITFC & SWITMC & SWITCF & SWITP & SS & ESP \\
\hline SWITFC & $-.33 *$ & & & & & & \\
SWITMC & $-.37 * *$ & .05 & & & & & \\
SWITCF & $-.53 * * *$ & $.42 * *$ & $.24 *$ & & & & \\
SWITP & $-.50 * * *$ & .17 & .09 & $.52 * * *$ & & & \\
SS & $.54 * * *$ & -.09 & $-.27 *$ & -.12 & $-.35 * *$ & & \\
ESP & $.76 * * *$ & $-.26 *$ & $-.32 *$ & $-.29 *$ & $-.40 * *$ & $.39 * *$ & \\
DEP & $.64 * * *$ & $-.25 *$ & -.01 & $-.32 *$ & $-.48 * * *$ & $.34 * *$ & $.53 * * *$ \\
\hline
\end{tabular}

Note: POFS = Perceptions of False Self Scale; SWITFC $=$ Say What I think around female classmates; SWITMC = Say what I think around male classmates; SWITCF = Say what I think around close friends; SWITP = Say what I think around parents; SS = Silencing the self; ESP = Externalised self perception; DEP = depressive symptoms. 


\section{GENERAL DISCUSSION}

\section{Scale Development}

A new false self scale was developed and tested through a series of three studies. Items were developed based on two sources: the current literature on false self and self silencing (Harter et al., 1996; Harter, Bresnick et al., 1997; Harter, Waters et al., 1997; Jack, 1991; Jack \& Dill, 1992), as well as through interviews with adolescents. A series of principal component analyses, along with an assessment of item content and clarity enabled an initial set of items to be reduced to a 16-item, 1 -factor perception of false self scale. This 1 -factor structure was verified with a confirmatory factor analysis, and the final scale demonstrated excellent internal reliability as well as criterion and convergent validity.

The POFS scale represents an advance in the measurement of perceptions of false self for adolescents for at least 3 reasons. First, the format of the POFS scale is easier for adolescents to understand. Second, the POFS scale is short and quick to administer; the POFS scale does not require individual administration, nor does it require multiple administrations to assess false self in multiple contexts. Third, the POFS scale is a macro-level measure of false self perception, which correlates highly with micro-level, relational context type measures.

It is important to highlight that the POFS scale does not represent a conflicting conceptualization of false self behaviour with previous research. Indeed, a large influence on the development of initial scale items came from existing theory and scales measuring false self and self-silencing behaviour. Rather, the POFS scale measures a generalised self perception where one feels false or phoney. When researchers are interested in false self behaviour across situations generally and want 
to encompass a variety of false self behaviours (voice, emotion, and appearance), the POFS scale may be more appropriate than the relational context scales or the multiple self procedure. But if researchers want to compare false self behaviour across different (but specific) contexts, then the already existing measures (e.g. SWIT) may be more appropriate. It is not uncommon for psychological phenomena to be assessed at both a general (macro) and a domain-specific level (micro) (see the Shapiro Control Inventory by Shapiro, 1994). Thus, these two measurement strategies are not necessarily mutually exclusive. In fact, study 3 showed that the micro- (SWIT) and macro-level scale (POFS) are strongly related.

False Self Behaviour and Maladjustment

Although previous research has found that false self behaviour is associated with maladjustment in general (Harter et al., 1996), very few studies have examined the relationship between false self and maladjustment across time. Supporting previous research perceptions of false self were concurrently associated with higher levels of maladjustment. When the relationship between perceptions of false self and maladjustment was assessed longitudinally, however, a unidirectional relationship was established. In particular, perceptions of false self did not predict changes in maladjustment across time, but maladjustment did predict increases in perceptions of false self across time. These results suggest that the direction of effect between maladjustment and perceptions of false self is primarily from the former to the latter. In other words, perceptions of false self appear to be prompted by increases in maladjustment.

In sum, across three studies, this chapter has described the development of the POFS scale for adolescents. Using a variety of techniques, the scale's reliability 
and validity was found to be very good, suggesting it is an appropriate measure to assess generalized false self perceptions in adolescents. Furthermore, perceptions of false self were longitudinally related to maladjustment, but the direction of effect was stronger from maladjustment to false self perception than the reverse. In the next chapter, generalized false self will be discussed in light of additional risk and vulnerability factors for maladjustment during adolescents, namely, stress, rumination, and sense of control. 


\section{Chapter 5: The Relationships Among Stress, Rumination, Sense of Control, Perceptions of False Self, and Maladjustment Across 10 Weeks}

The current chapter extends upon findings presented in Chapter 2 by assessing the interrelationships among stress, false self, sense of control, rumination, and maladjustment across time. Recall there were five major findings from Chapter 2, which assessed the interrelationships among stress, rumination, sense of control, and depressive symptoms. First, the gender difference in rumination occurred at the same age as the gender difference in depressive symptoms-at age 13. Second, rumination mediated rather than moderated the relationship between stress and depressive symptoms. Third, rumination was not as predictive as sense of control of depressive symptoms. Fourth, rumination and sense of control did not interact to predict depressive symptoms. Instead, rumination predicted depressive symptoms by predicting decreases in perceived control. And last, the interrelationships among stress, rumination, sense of control, and depressive symptoms were similar for males and females; however the relationships appeared to stabilize slightly in older preadolescents.

In large part, the current study was conducted to overcome two of the major limitations of Study $1^{28}$. First, Study 1 only sampled preadolescents $(9-13$ years old), so the relationships among stress, sense of control, rumination, and depressive symptoms could not be assessed in adolescents. Because adolescence is often conceptualized as the age at which gender differences in risk, vulnerability, and maladjustment emerge (Allgood-Merten et al., 1990; Twenge \& Nolen-Hoeksema, 2002), involving both preadolescents and adolescents in a sample is critical for a full

\footnotetext{
${ }^{28}$ As described in Chapter 4, another major goal of Study 2 was to develop and validate the Perceptions of False Self scale (POFS).
} 
test of RST. In Study 1, a trend emerged whereby females started to ruminate more than males at the same time that females began reporting more depressive symptoms than males-at 13 years old. In the current study, a larger age range was recruited to test whether the findings of Chapter 2 could be replicated. It was predicted, based on previous research, that there would be no gender differences in preadolescents, however, it was expected that female adolescents would report more stress, rumination, and maladjustment, and lower perceived control over their lives than male adolescents. In short, significant age by gender interactions were expected on stress, sense of control, rumination, and maladjustment ${ }^{29}$.

The second major limitation of Study 1 was the use of concurrent data. With concurrent data it is not possible to disentangle the direction of effect among variables. The full mediation model presented in Chapter 2 assumed, based on theoretical models of depression, that stress, rumination, and sense of control could prompt increases in depressive symptoms during preadolescence. That is, it was assumed that the direction of effect was from stress, rumination, and sense of control to depressive symptoms. The proposed mediational model was tested in this study both concurrently and longitudinally using structural equation modeling. Concurrently, I predicted the mediational model would be replicated. Longitudinally, the mediational model was tested using a cross lag panel design, called the direction of effects model. In order for the mediational model to be supported longitudinally in the direction of effect model, three sets of predictions required support. First, I predicted that higher initial levels of stress and lower sense of control would predict increases in later maladjustment, while controlling for initial levels of maladjustment.

\footnotetext{
${ }^{29}$ Age and gender trends were not assessed for perceptions of false self as these findings have been reported in Chapter 4, using the same participants.
} 
Second, I predicted that initial stress would predict increases in later rumination, and decreases in later perceived control across time, while controlling for initial levels of rumination and sense of control. And third, I predicted that rumination would predict lower perceived control across time, while initial levels of perceived control were controlled. Therefore, the two-variable mediating path identified in Chapter 2, namely originating with stress and passing through rumination and sense of control to maladjustment was tested; however it was unknown whether this mediational path would hold up in a more stringent longitudinal test.

In addition to these pathways to maladjustment, multiple alternative directions of effect could have been stipulated and are indeed possible (Arnow et al., 2004). Testing all possible directions of effect was not performed in Chapter 2 because doing so would have greatly inflated the chances of type 1 error. In addition, theoretical reasons for testing all possible mediational paths were lacking. One alternative direction of effect, suggested by previous researchers, is that there may be reciprocal relationships among these variables, where depressive symptoms also play a causal role on stress, rumination, and sense of control (Conway et al., 2004; Gibb \& Alloy, 2006; Nolen-Hoeksema, Stice et al., 2007; Weir et al., 2006). NolenHoeksema and Stice et al. (2007) are some of the few researchers who have tested alternative directions of effect in adolescent samples; however their data only includes female adolescents. Nolen-Hoeksema et al.'s results suggest that across four years depression and rumination predict increases in one another. In other words the relationship between rumination and depressive symptoms is bidirectional. This result is consistent with other data found with adult samples (Weir et al., 2006). Interestingly, in all of these studies, the general trend of effect was stronger from 
psychopathology to rumination than the reverse. To test the direction of effect among rumination and maladjustment, a cross lag model only involving rumination and maladjustment was conducted, in an attempt to replicate Nolen-Hoeksema, Stice et al.'s findings. Then additional risk and vulnerability factors were included in the model to assess the direction of effect among stress, vulnerability, and maladjustment, and to test how the relationship between rumination and maladjustment may be affected by additional variables. Although this latter analysis was exploratory, drawing upon the results of Conway et al., (2004), Gibb and Alloy (2006), and NolenHoeksema and Stice et al.), I predicted that the relationships among risk, vulnerability, and maladjustment would be bidirectional. Furthermore, I predicted that within these bidirectional relationships, the link from maladjustment to risk and vulnerability would be stronger than the link from risk and vulnerability to maladjustment.

There were two further goals of the current study. First, an additional risk factor for the development of depressed mood, false self perceptions, was included in the analysis. False self perceptions, as described in Chapter 4, are created when an individual perceives their behaviour to be false or phoney. In other words, false self perceptions occur when an individual considers their behaviour to be inauthentic. Chapter 4 showed that perceptions of false self are related to maladjustment, and that longitudinally maladjustment predicts increases in false self perceptions. In the current chapter, the relationships among false self, stress, sense of control, and rumination are explored. It was predicted that higher perceptions of false self will be associated with higher levels of stress, rumination, and lower perceived control. Longitudinally, no 
predictions were made as to whether false self perceptions could predict changes in stress, sense of control, or rumination as these analyses were exploratory.

Second, the diathesis-stress model of depression predicted by RST was tested using longitudinal data. Specifically, it was tested whether stress interacted with rumination to predict maladjustment across time. Based on the findings in Chapter 2, I predicted that the diathesis-stress model asserted by RST would not be supported. Comparative diathesis-stress models were also tested whereby sense of control and perceptions of false self were stipulated as vulnerability factors.

In addition to these diathesis-stress models, recall from Chapter 1 that RST asserts that if an individual ruminates while feeling distressed, rumination will prolong and intensify maladjustment (Nolen-Hoeksema, 1991). This hypothesis can be directly tested using a moderation analysis. Within a moderation framework this hypothesis suggests that rumination would moderate the relationship between initial and later maladjustment. In other words, follow-up maladjustment would be most intense when individuals who are distressed at baseline ruminate about their problems. Recall from Chapter 1 that such a hypothesis has been supported using survey data in adult samples (Nolan et al., 1998), however this hypothesis has not been tested in adolescent samples. In the current chapter I predicted that rumination would moderate the relationship between initial and later maladjustment, that is, rumination would exacerbate maladjustment across time.

To summarize, the current study extends the methodology presented in Chapter 2 by collecting data from both preadolescent and adolescent populations across 10 weeks, as well as including an additional comparative vulnerability factor for maladjustment (false self). There were three overarching goals of the current study, 
first, to test for age and gender trends among the measured variables using a larger age range than in Chapter 2. It was predicted that there would be no gender differences on any variables for preadolescents; however, adolescent females were expected to report more stress, rumination, maladjustment, and less perceived control over their lives than adolescent males. Second, the interrelationships among all variables were explored, both concurrently and longitudinally. The moderation results from chapter 2 were tested for longitudinal replication, that is, I predicted that the stress-diathesis model would not be supported longitudinally. However, it was predicted that rumination would moderate the relationship between maladjustment across time based on the results of Nolan et al. (1998). It was also predicted that the mediation model established in Chapter 2 would be supported both concurrently and longitudinally. And last, I predicted that there would be reciprocal relationships among risk, vulnerability and maladjustment across the 10 weeks.

\section{Method}

Participants and Procedure

Recall from Chapter 4 that 195 adolescents (68 males, 127 females) participated during data collection across 10 weeks. During the same sessions described in Chapter 4 where participants completed the POFS, RMCA, and CDI, participants also completed measures of stress, sense of control, and rumination. Recall there were 32 (12 males, 20 females) eleven year olds, 62 (26 males, 36 females) twelve year olds, 31 (7 males, 24 females) thirteen year olds, 45 (15 males, 30 females) fourteen year olds, and 25 (8 males, 17 females) fifteen year olds. As described in Chapter 4, age was broken down into two categories, less than 13 years (preadolescents), and greater than or equal to 13 years old (adolescents). In the final 
sample, 64\% were European New Zealanders, 13\% were Maori, 7.5\% were Asian, 4.5\% were Pacific Islanders, and 11\% classified their ethnicity as 'Other'. The procedure was identical to that described in previous chapters.

Materials

Stress $^{30}$. Stress was measured using the Everyday Life Events Scale for Children (ELESC; Jose, Cafasso, \& D’Anna, 1994). This scale was used instead of the stress measure constructed for Study 1 because the reliability and validity of the ELESC has been established (Jose et al., 1994). The ELESC scale assesses the presence and severity of everyday stressful events for children and adolescents (e.g."You misplaced or lost something"; "You were avoided by someone"; "Not enough time for relaxation"). In total, 40 different everyday stressors were assessed. Everyday life events rather than major life events were assessed because the former have been found to predict maladjustment better than the latter (DeLongis, 1982). The ELESC is structured so that for each event, an adolescent indicates whether the event has happened to them in the last month or not. For all events that happened, the adolescent then indicates how much of a problem that event was $(0=$ not a problem, $1=$ a little, $2=$ some, $3=$ a lot $)$. In this study, the severity score was summed to create a total stress score; stress scores can range between 0 - 120. This method was adopted because previous studies have shown that summing the severity scores is a better indicator of stress than summing the frequency of events (Jose et al., 1994). In this sample, the scale exhibited excellent internal reliability at both baseline, $(\alpha=.91)$ and 10 weeks $(\alpha=.93)$.

\footnotetext{
${ }^{30}$ The Everyday Life Events Scale used in this study is presented in Appendix K
} 
Perceptions of False Self. The 16-item Perceptions of False Self Scale (POFS) was used to assess the extent to which an adolescent perceives their behaviour to be false. A full description of the scale's development was discussed in Chapter 4.

Rumination. The 16-item version of the ruminative response subscale from the RSQ (Nolen-Hoeksema et al., 1993) was used to assess rumination in this study. The RSQ has been described in Chapter 2. In this sample, the scale was internally reliable at both baseline, $(\alpha=.86)$ and 10 weeks $(\alpha=.89)$.

Sense of Control and Depression were assessed using the Overall Sense of Control scale (OSOC; Weir \& Jose, 2006a) and the Children's Depression Inventory (CDI; Kovacs, 1992) described in Chapter 2. Anxiety was assessed using the Revised Children's Manifest Anxiety scale (RMCA; Reynolds \& Richmond, 1997) described in Chapter 4. In this sample, all three scales exhibited excellent internal reliability at both baseline, ( $\alpha_{\mathrm{s}}=.88$ to .92$)$ and 10 weeks ( $\alpha_{\mathrm{s}}=.90$ to .92$)$. As described in Chapter 4, anxious and depressive symptoms were highly correlated and were therefore linearly combined to represent a maladjustment variable.

\section{Results}

Analytic Strategy

The analytic strategy of the current chapter was similar to that of Chapter 2.

The first goal was to explore the developmental trends and test for gender differences on stress, sense of control, rumination, and maladjustment-a repeated-measures MANOVA was conducted to identify relevant findings. Second, the concurrent and longitudinal relationships among all variables were assessed using zero-order correlations. Third, multiple regression analyses were conducted to test the moderation models described above. Fourth, the mediational model proposed in 
Chapter 2 was tested for replication using concurrent measures. And last, the directions of effect were tested. A longitudinal cross lag model involving only rumination and maladjustment was tested first. Building on this model, stress, sense of control, and perceptions of false self were then included in the model to allow for comparative predictors of maladjustment. This second directions of effect model also enabled a longitudinal test of the mediational model described in Chapter 2.

Developmental Trends of Stress, Sense of Control, Rumination, and Maladjustment

To explore age and gender differences, a 2 (age: preadolescents, adolescents) x 2 (gender: male, female) x 2 (time: baseline, 10 weeks) repeated measures MANOVA was conducted. Stress, sense of control, rumination, and maladjustment were stipulated as the dependent variables, and time was stipulated as the withinsubjects factor. It was predicted that significant age by gender interactions on all variables would be found. Specifically, it was predicted that there would be no difference between male and female preadolescents on any of the variables, however adolescent females would report significantly more stress, rumination, maladjustment, and lower perceived control than adolescent males.

Multivariate Results. Within-subjects multivariate tests indicated a significant effect of time, $F(4,188)=54.03, p<.001$, partial $\eta^{2}=.54$. The time $\mathrm{x}$ gender, $F(4$, $188)=.03, p=.17$, partial $\eta^{2}=.03$, the time $\mathrm{x}$ age, $F(4,188)=1.73, p=.15$, partial $\eta^{2}=.04$, and the time $\mathrm{x}$ gender $\mathrm{x}$ age, $F(4,188)=.01, p=.96$, partial $\eta^{2}<$ .01 , interactions did not reach significance. Thus, only the univariate effects of time were examined.

Multivariate tests for between-subjects results indicated a significant multivariate effect of gender, $F(4,188)=6.29, p<.001$, partial $\eta^{2}=.12$, and a 
significant multivariate age $\mathrm{x}$ gender interaction, $F(4,188)=3.25, p<.01$, partial $\eta^{2}$ $=.07$. The multivariate effect of age was not significant, $F(4,188)=.59, p=.67$, partial $\eta^{2}=.01$. Univariate tests for gender and the age by gender interaction were examined.

Univariate Results. Regarding univariate effects for time (Table 5.1), both stress and sense of control significantly increased, whereas rumination decreased across the 10 weeks. Notably, the increase in reported stress was large compared to the changes in the other measured variables. The large increase in stress was most likely the result of the timing of data collection. The second phase of data collection was conducted near the end of the school year and, hence, was close to end of year exams for adolescents. At this time, stress levels would have been high. The improvement in self-reported adjustment (rumination, sense of control) was possibly the result of repeated exposure to the scales, which has been evident in previous research (e.g. Twenge \& Nolen-Hoeksema, 2002).

Table 5.1. Within Subject Effects on Stress, Sense of Control, Rumination, and Maladjustment

\begin{tabular}{lcccccc}
\hline \multicolumn{7}{c}{ Within subject effects of time $(d f=1,191)$} \\
\hline \multicolumn{7}{c}{ Baseline } \\
$M$ & $S D$ & $M$ & $S D$ & $F_{\text {time }}$ & Partial $\eta^{2}$ \\
\hline Stress & 25.32 & 17.66 & 41.46 & 25.47 & $151.68 * * *$ & .44 \\
Control & 62.47 & 9.45 & 63.71 & 10.00 & $6.52 * *$ & .03 \\
Rumination & 40.89 & 10.42 & 39.28 & 11.03 & $7.61 * *$ & .04 \\
Maladjustment & 00.00 & 1.90 & 00.00 & 1.89 & .84 & $<.01$ \\
\hline
\end{tabular}

$* * p<.01 * * * p<.001$.

Regarding the between-subjects univariate effects (Table 5.2), females reported significantly higher levels of stress, rumination, and maladjustment than males. Males and females reported similar levels of perceived control. Regarding the univariate age by gender interactions, significant effects were evident for perceived control (Figure 5.1), and maladjustment (Figure 5.2). The age $\mathrm{x}$ gender interactions 
for stress $(p=.057)$ and rumination $(p=.068)$ were marginal. As can be seen in

Table 5.2, these interactions were the result of male and female preadolescents reporting similar levels of stress, perceived control, rumination, and maladjustment, however female adolescents reported less perceived control, and more stress, rumination, and maladjustment than adolescent males, thus supporting the predictions of this study. Similar to Chapter 2, however, effect sizes were small.

Table 5.2. Between-Subject Effects on Stress, False Self, Control, Rumination, and Maladjustment

\begin{tabular}{|c|c|c|c|c|c|c|c|c|}
\hline \multicolumn{9}{|c|}{ Between Subject Effects $(d f=1,191)$} \\
\hline & \multicolumn{2}{|c|}{ Preadolescents } & \multicolumn{2}{|c|}{ Adolescents } & \multirow[t]{3}{*}{$F_{\text {gender }}$} & \multirow[t]{3}{*}{ Partial $\eta^{2}$} & \multirow[t]{3}{*}{$F_{\text {agexgender }}$} & \multirow[t]{3}{*}{ Partial $\eta^{2}$} \\
\hline & Male & Fem & Male & Female & & & & \\
\hline & $M(S . E)$ & $M(S . E)$ & $M(S . E)$ & $M(S . E)$ & & & & \\
\hline \multirow[t]{2}{*}{ Stress } & 30.91 & 33.76 & 24.01 & 38.39 & $8.21 * *$ & .04 & $3.67 \sim$ & .02 \\
\hline & $(3.22)$ & $(2.66)$ & $(3.63)$ & $(2.36)$ & & & & \\
\hline \multirow[t]{2}{*}{ Con } & 62.79 & 65.06 & 67.15 & 59.98 & 3.23 & .02 & $12.02 * * *$ & .06 \\
\hline & $(1.46)$ & $(1.20)$ & $(1.64)$ & $(1.07)$ & & & & \\
\hline \multirow[t]{2}{*}{ Rum } & 37.37 & 41.62 & 33.55 & 43.09 & $22.96 * * *$ & .11 & $3.38 \sim$ & .02 \\
\hline & (1.54) & $(1.27)$ & $(1.74)$ & (1.13) & & & & \\
\hline \multirow[t]{2}{*}{ MA } & -.08 & -.27 & -.80 & .60 & $5.05 *$ & .03 & $8.78 * *$ & .04 \\
\hline & $(.29)$ & $(.23)$ & $(.32)$ & $(.21)$ & & & & \\
\hline
\end{tabular}

Note: Con $=$ Sense of Control; Rum $=$ Rumination; MA = Maladjustment. $\sim_{p}<.10, *_{p} \leq .05, *_{p} \leq$ $.01 * * * p \leq .001$

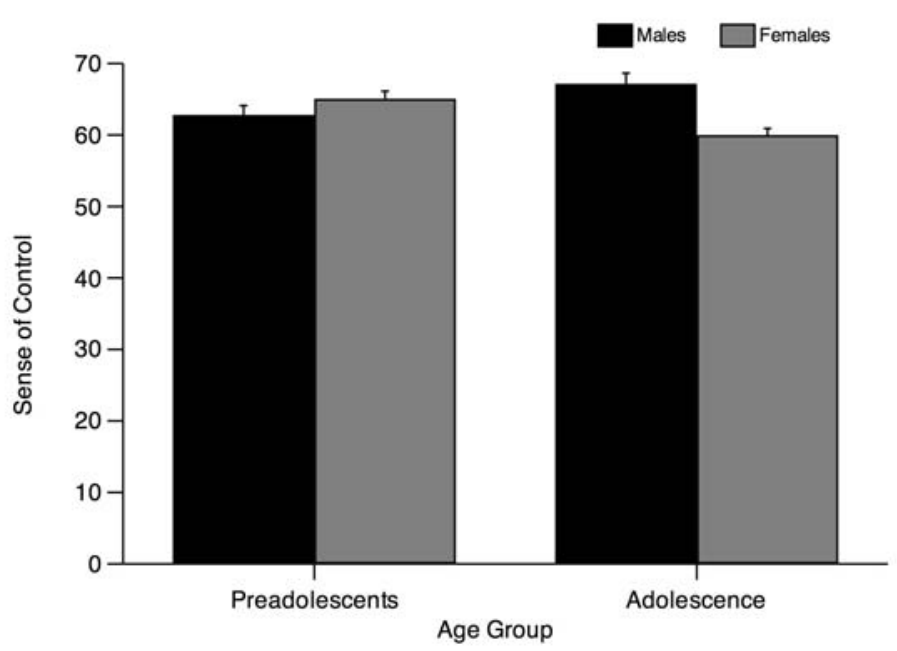

Figure 5.1. Trends by age and gender on sense of control 


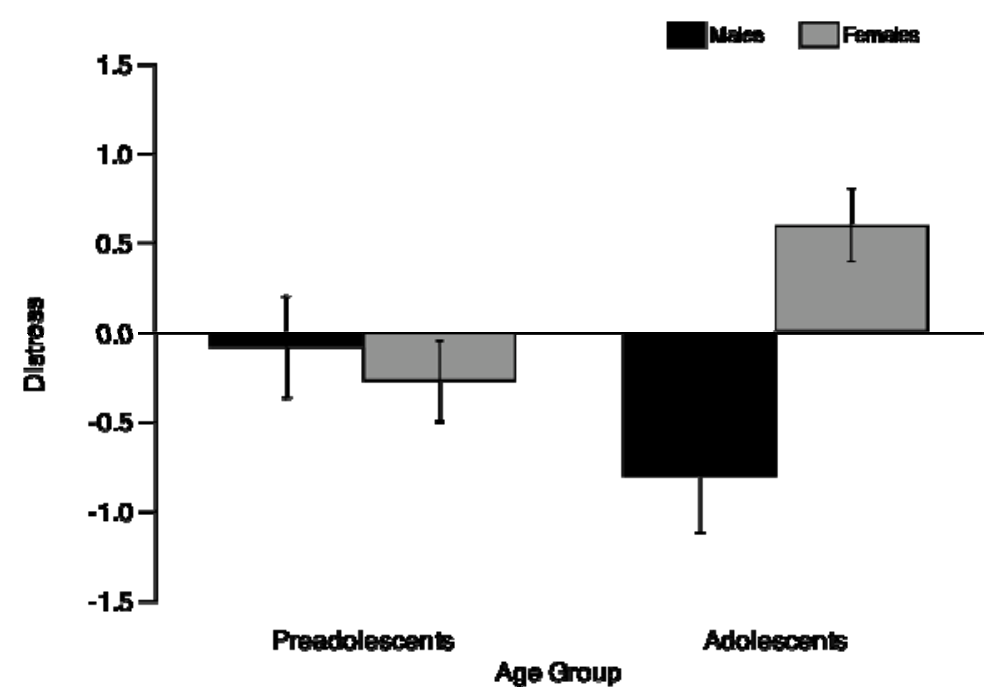

Figure 5.2. Trends by age and gender on maladjustment

Relationships Among Stress, False Self, Sense of Control, Rumination, and Maladjustment

Concurrent Correlations. Concurrent correlations among stress, perceptions of

false self, sense of control, rumination, and maladjustment were all within the moderate to strong range (Cohen, 1992), and were in the expected direction (Table 5.3). For example, adolescents who reported higher stress, perceptions of false self, and rumination and less perceived control were more likely to report greater maladjustment.

Table 5.3. Concurrent Correlations Among Stress, False Self Perceptions, Sense of Control, Rumination, and Maladjustment.

\begin{tabular}{lccccc}
\hline & Stress & False Self & Control & Rum & MA \\
\hline Stress & - & $.50 * * *$ & $-.55 * * *$ & $.57 * * *$ & $.73 * * *$ \\
False Self & $.48 * * *$ & - & $-.54 * * *$ & $.49 * * *$ & $.68 * * *$ \\
Control & $-.59 * * *$ & $-.56 * * *$ & - & $-.51 * * *$ & $-.65 * * *$ \\
Rum & $.56 * * *$ & $.53 * * *$ & $-.51 * * *$ & - & $.62 * * *$ \\
MA & $.74 * * *$ & $.70 * * *$ & $-.73 * * *$ & $.65 * * *$ & - \\
\hline
\end{tabular}

Note: Time one correlations are above the diagonal, Time two correlations are below the diagonal. $* * * p<.001$. Rum $=$ Rumination $;$ MA $=$ Maladjustment. 
Longitudinal Associations. Similar to the results with concurrent data, all

variables were interrelated across time to a moderate to strong degree (Table 5.4).

Furthermore, for all variables, test-retest reliability was high, indicating that the constructs are relatively stable across time.

Table 5.4. Longitudinal Relationships Among Stress, False Self Perceptions, Sense of Control,

Rumination, and Maladjustment.

\begin{tabular}{lccccc}
\hline & Stress T1 & POFS T1 & Con T1 & Rum T1 & MA T1 \\
\hline Stress T2 & $.77 * * *$ & & & & \\
POFS T2 & $.52 * * *$ & $.84 * * *$ & & & \\
Con T2 & $-.56 * * *$ & $-.52 * * *$ & $.75 * * *$ & & \\
Rum T2 & $.47 * * *$ & $.44 * * *$ & $-.39 * * *$ & $.76 * * *$ & \\
MA T2 & $.66 * * *$ & $.62 * * *$ & $-.59 * * *$ & $.58 * * *$ & $.87 * * *$ \\
\hline
\end{tabular}

Note: POFS = Perceptions of False Self; Con = Sense of Control; Rum = Rumination; MA = Maladjustment; $\mathrm{T} 1=$ Baseline Variables; $\mathrm{T} 2=$ Variables at 10 weeks. Correlations along the diagonal reflect test-retest correlations. $* * * p<.001$.

Moderation Analyses

Does stress interact with vulnerability to predict increases in maladjustment across 10

weeks? Three separate moderation analyses were conducted to test the diathesis-stress model asserted by RST across time. One moderation analysis was conducted for each vulnerability factor (false self, sense of control, rumination) ${ }^{31}$. In all cases the recommendations of Baron and Kenny (1986) and Holmbeck (1997) were followed. These moderation analyses were conducted in an attempt to replicate and extend findings from Chapter 2, and to test whether perceptions of false self interacted with stress to predict increases in maladjustment across time. It was predicted that the stress-diathesis model for rumination and sense of control would not be supported longitudinally based on the results presented in Chapter 2. No prediction was made for the stress-diathesis model involving perceptions of false self.

\footnotetext{
31 A moderation analysis where the vulnerability variables interacted was not conducted because no support was found for such an exploratory model in Chapter 2.
} 
For each of the three moderation analyses, baseline maladjustment was entered in the first step of the regression. The main effects were entered into the second step (stress, and vulnerability: rumination/sense of control/false self). Stress, false self, rumination, and sense of control were all centred prior to creating the interaction terms to reduce multicollinearity (Howell, 2002). In the third step, the two-way interaction was entered (stress $\mathrm{x}$ vulnerability). As can be seen in Table 5.5, results were similar across the three stress-diathesis models.

Table 5.5. Results of Stress-Diathesis Moderation Analyses

\begin{tabular}{|c|c|c|c|c|c|}
\hline \multicolumn{6}{|c|}{ Stress $\mathrm{x}$ Rumination } \\
\hline & & $B$ & $S E B$ & $\beta$ & $\Delta R^{2}$ \\
\hline \multirow[t]{2}{*}{ Step1 } & & & & & $.75 * * *$ \\
\hline & MA & .86 & .04 & $.87 * * *$ & \\
\hline \multirow[t]{3}{*}{ Step2 } & & & & & $<.01$ \\
\hline & Stress & $<.01$ & .01 & .04 & \\
\hline & Rum & .01 & .01 & .06 & \\
\hline \multirow[t]{2}{*}{ Step3 } & & & & & $<.01$ \\
\hline & $\mathrm{S} \times \mathrm{R}$ & $<.01$ & $<.01$ & $<.01$ & \\
\hline \multicolumn{6}{|c|}{ Stress x Sense of Control } \\
\hline & & $B$ & $S E B$ & $\beta$ & $\Delta R^{2}$ \\
\hline \multirow[t]{2}{*}{ Step1 } & & & & & $.75 * * *$ \\
\hline & MA & .86 & .04 & $.87 * * *$ & \\
\hline \multirow[t]{3}{*}{ Step2 } & & & & & $<.01$ \\
\hline & Stress & .01 & .01 & .05 & \\
\hline & Control & $<-.01$ & .01 & -.05 & \\
\hline \multirow[t]{2}{*}{ Step 3} & & & & & $<.01$ \\
\hline & $\mathrm{S} \times \mathrm{C}$ & $<.01$ & $<.01$ & -.02 & \\
\hline \multicolumn{6}{|c|}{ Stress x False Self Perception } \\
\hline & & $B$ & $S E B$ & $\beta$ & $\Delta R^{2}$ \\
\hline \multirow[t]{2}{*}{ Step1 } & & & & & $.75 * * *$ \\
\hline & MA & .86 & .04 & $.87 * * *$ & \\
\hline \multirow[t]{3}{*}{ Step2 } & & & & & $<.01$ \\
\hline & Stress & .01 & .01 & .06 & \\
\hline & False & .01 & .01 & .06 & \\
\hline \multirow[t]{2}{*}{ Step 3} & & & & & $<.01$ \\
\hline & $\mathrm{S} \times \mathrm{F}$ & $<.01$ & $<.01$ & $<-.01$ & \\
\hline
\end{tabular}

Note. $\mathrm{MA}=$ Maladjustment at baseline; $\mathrm{S}=$ Stress at baseline; $\mathrm{R}=$ Rumination at baseline; $\mathrm{C}=$ Sense of control at baseline. $\mathrm{F}=$ Perceptions of false self at baseline; For ease of interpretation, $B, S E B$, and $\beta$ are presented at the step the variable was entered. $* * * p \leq .001$. 
In all three models, maladjustment at baseline significantly predicted maladjustment 10 weeks later, explaining $75 \%$ of the variance in 10 week maladjustment. Likewise, in all three models, neither the second nor third steps explained additional variance in maladjustment at 10 weeks. Overall, the results of the three moderation analyses do not support the stress-diathesis model of maladjustment, as predicted. More specifically, neither stress nor vulnerability (either alone or in combination) predicted maladjustment across time while baseline maladjustment was controlled.

Does rumination moderate the relationship between initial and later maladjustment? Another moderation analysis was conducted, which tested whether rumination moderates the relationship between initial and later maladjustment as RST predicts. To test this prediction, maladjustment was entered into the first step of a regression, next rumination (centred) was entered into the next step of the regression, and last, the maladjustment $\mathrm{x}$ rumination interaction term was entered in the last step of the analysis $^{32}$. Contrary to the predictions of RST, rumination did not interact with initial levels of maladjustment to predict maladjustment across time (Table 5.6).

To explore whether sense of control and perceptions of false self exacerbated the relationship between initial and later maladjustment across time, two further moderation models were tested. Similarly to the results with rumination, neither sense of control nor perceptions of false self exacerbated maladjustment across time (Table 5.6). Overall, none of these variables-rumination, sense of control, and perceptions of false self-exacerbated maladjustment across time.

\footnotetext{
${ }^{32}$ The maladjustment variable did not require centring because it is the linear combination of depressive and anxious symptoms, and therefore already has a mean of 0 .
} 
Table 5.6. Results of Moderation Analyses Where Vulnerability Factors Are Hypothesized to Exacerbate Maladjustment

\begin{tabular}{|c|c|c|c|c|c|}
\hline \multicolumn{6}{|c|}{ Maladjustment x Rumination } \\
\hline & & $B$ & $S E B$ & $\beta$ & $\Delta R^{2}$ \\
\hline \multirow[t]{2}{*}{ Step1 } & & & & & $.75 * * *$ \\
\hline & MA & .87 & .04 & $.87 * * *$ & \\
\hline \multirow[t]{2}{*}{ Step2 } & & & & & $<.01$ \\
\hline & Rum & .01 & .01 & .07 & \\
\hline \multirow[t]{2}{*}{ Step3 } & & & & & $<.01$ \\
\hline & $M A \times R$ & $<.01$ & $<.01$ & .02 & \\
\hline \multicolumn{6}{|c|}{ Maladjustment x Sense of Control } \\
\hline & & $B$ & $S E B$ & $\beta$ & $\Delta R^{2}$ \\
\hline \multirow[t]{2}{*}{ Step1 } & & & & & $.75 * * *$ \\
\hline & MA & .86 & .04 & $.87 * * *$ & \\
\hline \multirow[t]{2}{*}{ Step2 } & & & & & $<.01$ \\
\hline & Control & -.01 & .01 & -.05 & \\
\hline \multirow[t]{2}{*}{ Step3 } & & & & & $<.01$ \\
\hline & $\mathrm{MA} \times \mathrm{C}$ & $<-.01$ & $<.01$ & -.04 & \\
\hline \multicolumn{6}{|c|}{ Maladjustment x Perceptions of False Self } \\
\hline & & $B$ & SEB & $\beta$ & $\Delta R^{2}$ \\
\hline \multirow[t]{2}{*}{ Step1 } & & & & & $.75 * * *$ \\
\hline & MA & .86 & .04 & $.87 * * *$ & \\
\hline \multirow[t]{2}{*}{ Step2 } & & & & & $<.01$ \\
\hline & POFS & .01 & .01 & .06 & \\
\hline \multirow[t]{2}{*}{ Step3 } & & & & & $<.01$ \\
\hline & MA $x$ FS & $<.01$ & $<.01$ & .01 & \\
\hline
\end{tabular}

Note; MA = Maladjustment at Baseline; $\mathrm{R}=$ Rumination at baseline; FS = Perceptions of False Self at baseline. $B, S E B$, and $\beta$ are presented at the step the variable was entered. $* * *_{p} \leq .001$.

\section{Mediational Model Replication}

The mediational model presented in Chapter 2 was tested concurrently for replication using structural equation modeling. Figure 5.3 shows the replication of the proposed mediational model using baseline data. Each path identified in Chapter 2 was also significant in this sample. However, the model fit indices fell well below acceptable standards on all variables except the sRMR and the $G F I: \chi^{2} / d f=15.79, G F I$ $=.96, A G F I=.62, \mathrm{~s} R M R=.04, R M S E A=.27$. 


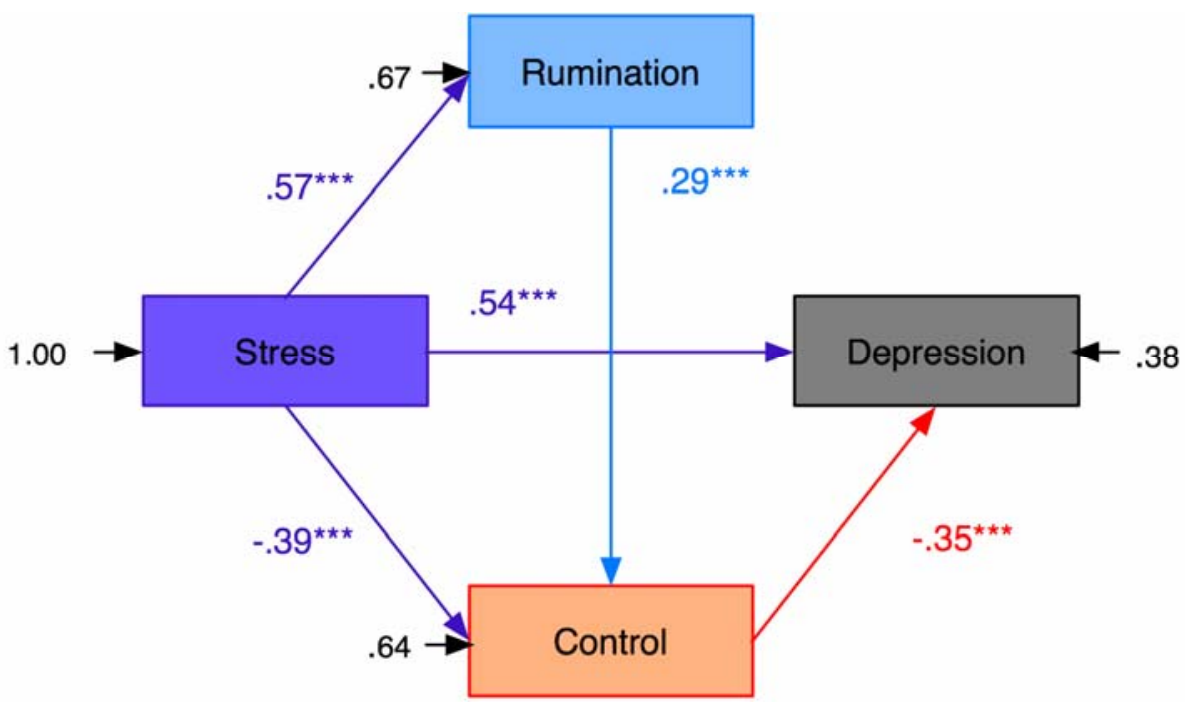

Figure 5.3. Mediation model replication

Modification indices suggested that paths from maladjustment to rumination and sense of control would improve the model fit. Thus, a further model (Figure 5.4) was run whereby stress and maladjustment predicted both sense of control and rumination, and rumination predicted sense of control. This modified model was fully saturated. All paths remained significant, except from stress to sense of control ( $p=$ .11). When the non significant path from stress to sense of control was removed, the model fit indices were acceptable, except for the $R M S E A, \chi^{2} / d f=2.45, G F I=.99$, $A G F I=.94, \mathrm{sRMR}=.02, \mathrm{RMSEA}=.09^{33}$.

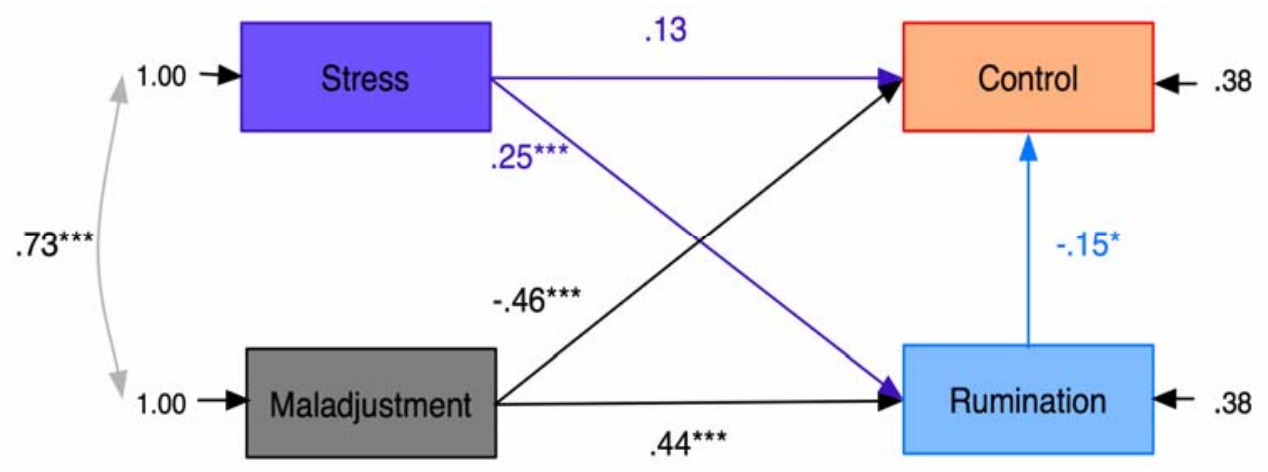

Figure 5.4. Modified mediation model

\footnotetext{
${ }^{33}$ A similar pattern of results was found when using follow up data
} 
In summary, the mediational model presented in chapter 2 was not supported concurrently. Instead, it appeared that stress and maladjustment predicted changes in vulnerability. These results suggest that the direction of effect is more complex than the original mediational model stipulates, for example, there may be reciprocal relationships among vulnerability and maladjustment ${ }^{34}$. To more fully explore this possibility, the longitudinal relationships among these measured variables and perceptions of false self were assessed next.

Directions of Effect Among Stress, Sense of Control, Rumination, Perceptions of False Self, and Maladjustment Across 10 weeks

Directions of effect among rumination and maladjustment. First, in an attempt to replicate the bidirectional relationship between rumination and depressive symptoms found by Nolen-Hoeksema, Stice et al., (2007), a cross lag panel design was conducted using structural equation modeling. Specifically, the model included the stability coefficients of rumination and maladjustment, as well the two cross lag paths, one from initial rumination to 10 week maladjustment and another from initial maladjustment to 10 week rumination. Concurrent error terms at baseline and follow up were allowed to correlate with each other, making the initial model fully saturated. Figure 5.5 shows that both rumination and maladjustment were stable across the 10 week period. However, initial levels of rumination did not predict 10 week maladjustment, as RST would predict. The path from initial maladjustment to 10 week rumination was marginally significant $(p=.08)$. When the non-significant path from rumination to maladjustment was removed, the path from initial maladjustment to 10 week rumination reached significance, $\beta=.13, p<.05$, and the model fit

\footnotetext{
${ }^{34}$ There were not enough degrees of freedom to insert exploratory reciprocal pathways between risk, vulnerability and maladjustment.
} 
indices reached acceptable levels, $\chi^{2} / d f=2.12, G F I=.99, A G F I=.95$, sRMR $=.02$, RMSEA $=.08$. In summary, the direction of effect between rumination and maladjustment appears to be from the latter to the former, which are contrary to the predictions of RST.

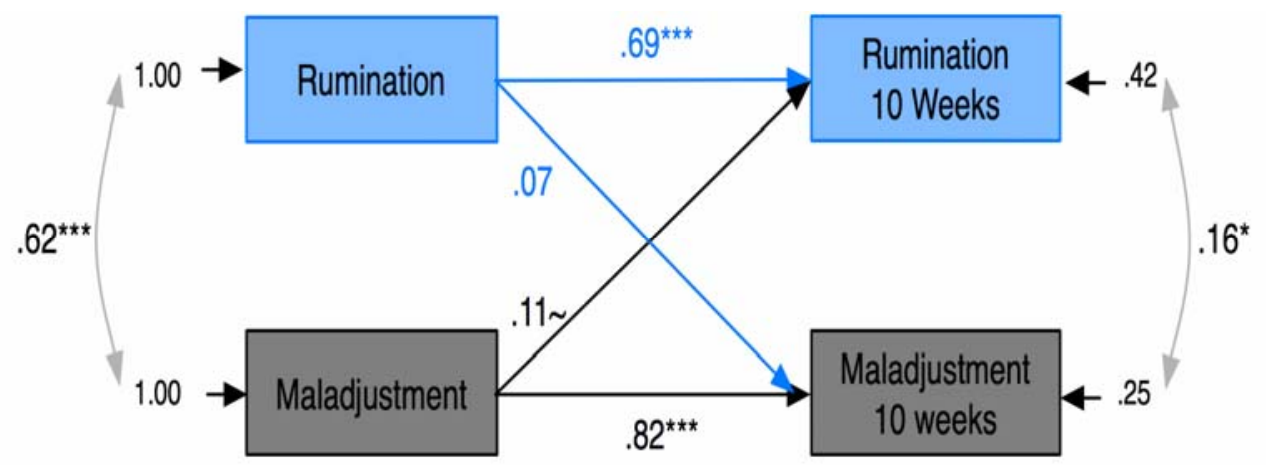

Figure 5.5.Direction of effect between rumination and maladjustment across 10 weeks

Directions of effect among all measured variables. Next I tested how stress, sense of control, and perceptions of false self might affect the relationship between rumination and maladjustment. To do this, I conducted another direction of effect model, with all measured variables included in the analysis. Given that very few studies have examined the causal directions among the variables measured here, an exploratory model was conducted following the recommendations of Kline (2005). Path modeling was conducted where all exogenous variables predicted all endogenous variables while the stability paths of each variable were controlled ${ }^{35}$. In other words, path modeling enabled multiple dependent variables to be predicted simultaneously while controlling for baseline levels of each variable.

Following the recommendations of Kline (2005), a base model was conducted where all structural paths were stipulated (i.e. a fully saturated model). Specifically, the initial model included the 5 autocorrelations (stability paths) of stress, perceptions

\footnotetext{
${ }^{35}$ Path models rather than latent variable models were conducted because of the current study's small sample size.
} 
of false self, sense of control, rumination, and maladjustment. A further 20 paths were stipulated; each variable at baseline was allowed to predict all other variables 10 weeks later. In addition to these direct paths, concurrent error terms at baseline and follow up were allowed to correlate with each other. Non-significant paths were then removed (pruned) to establish model fit indices (Kline). The pruning process involved removing each non-significant path from the model individually, from the least significant to the most. At each stage of the model pruning process, the model was examined to identify any fluctuations in coefficients, psi values, or error terms. Fluctuations would indicate the presence of problematic multicollinearity. Also, pruning the model in this manner allowed for other marginal paths to become significant. This latter consideration was especially important in light of the relatively small sample size of the current study.

From the initial fully saturated model, named the base model, eight significant paths were identified, 5 of which were stability coefficients and 3 of which were crosslag paths. The stability coefficients for stress, $\beta=.60, p<.001$, false self, $\beta=.67, p$ $<.001$, sense of control, $\beta=.53, p<.001$, rumination, $\beta=.70, p<.001$, and maladjustment, $\beta=.75, p<.001$, were all high. The significant cross-lag paths were from baseline maladjustment to stress at 10 weeks, $\beta=.22, p<.01$, from baseline maladjustment to false self at 10 weeks, $\beta=.22, p<.01$, and from baseline maladjustment to decreased sense of control at 10 weeks, $\beta=-.24, p<.001$. Interestingly, the path identified in Figure 5.5 from maladjustment to rumination was no longer significant, $\beta=.10, p=.24$.

Next, the model was pruned, using the procedure described above. In total, the pruning process deleted 18 estimated parameters. Thus, 18 degrees of freedom 
were freed during this process. No additional paths became significant while pruning. The identified cross lag paths from the base model, as well as the stability coefficients, remained significant after pruning. This pruned model (Figure 5.6) fit these data well, $\chi^{2} / d f=1.27, G F I=.98, A G F I=.93, \mathrm{sRMR}=.06, R M S E A=.04$. Furthermore, modification indices indicated that adding or deleting additional paths would not improve the model fit. In sum, these results suggest that maladjustment predicts increases in stress and perceptions of false self, as well as decreases in perceived control across time. However none of these variables predicted increases in maladjustment across time.

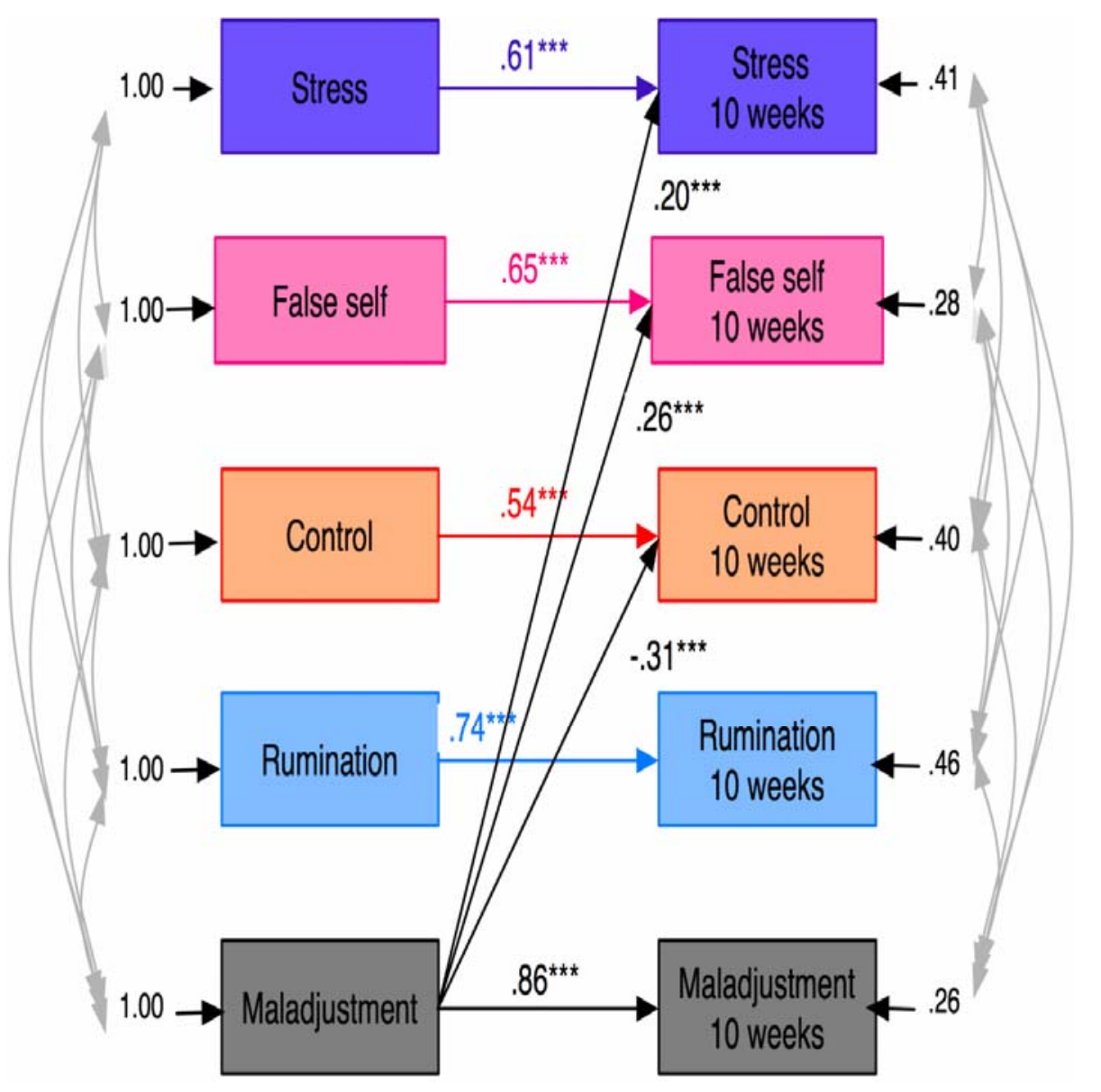

Figure 5.6. Pruned direction of effects model for the full sample. 
This direction of effect analysis also allowed for a longitudinal test of the mediational model presented in Chapter 2. Interestingly, no support for the mediational model presented in Chapter 2 was obtained from this direction of effects model. For the proposed mediational model to be supported longitudinally, additional cross lag paths would have had to be identified, for example, from baseline stress to rumination, from rumination to sense of control, and from sense of control to maladjustment. However, no cross-lag paths were identified from risk or vulnerability factors across time, contrary to predictions. Instead, it appeared that maladjustment leads to increased risk and vulnerability rather than the reverse.

Next, it was tested if this pruned model fit these data equally well across age and gender (i.e. moderated mediation). It was predicted that the model would fit these data for males and females equally well. However, based on the findings in Chapter 2 it was predicted that the model fit indices would be slightly better for adolescents than preadolescents. First, the model was tested on preadolescents $(\mathrm{N}=$ 94) and adolescents $(\mathrm{N}=101)$ separately. For preadolescents, all eight paths remained significant except for the path from baseline maladjustment to stress at 10 weeks, which became marginally significant, $\beta=.15, p=.06$. The model fit indices for this model fell below acceptable standards on the $A G F I$ and the $\mathrm{s} R M R, \chi^{2} / d f=$ 1.57, GFI $=.94, A G F I=.83, \mathrm{sRMR}=.07, R M S E A=.08$. Modification indices were checked; however no additional paths were suggested to be deleted or added to improve the model's fit. For adolescents, all eight paths were significant, however the model fit indices fell slightly below acceptable standards on the $A G F I, \chi^{2} / d f=1.44$, $G F I=.95, A G F I=.86, \mathrm{~s} R M R=.04, R M S E A=.06$. Again, modification indices did not indicate changing any additional paths to improve the model fit indices. 
Separate models were also run for males $(\mathrm{N}=68)$ and females $(\mathrm{N}=127)$.

For males, all stability paths were significant; however the only significant cross lag path identified was from baseline maladjustment to sense of control 10 weeks later. Neither the cross lag path from baseline maladjustment to stress at 10 weeks, $\beta=.02$, $p=.85$, nor false self at 10 weeks, $\beta=.14, p=.12$, reached significance. Although the model did not fit these data especially well, $\chi^{2} / d f=1.38, G F I=.94, A G F I=.80$, $\mathrm{s} R M R=.06, R M S E A=.07$, modification indices did not suggest any possible improvements. It is possible that the model did not adequately meet standards because of the sample size. For females, all eight paths were significant, and the model fit these data better, $\chi^{2} / d f=1.25, G F I=.97, A G F I=.90$, sRMR $=.06, R M S E A=.04$. Modification indices suggested including a pathway from baseline maladjustment to rumination at 10 weeks would significantly improve the model. A second model for females was run, and the path from baseline maladjustment to 10 week rumination was significant, $\beta=.24, p=.001$. This model fit the female data very well, $\chi^{2} / \mathrm{d} f=$ $.76, G F I=.98, A G F I=.94, \mathrm{~s} R M R=.02, R M S E A<.01$.

In summary, when broken down by age and gender, very few differences were identified. For females, an additional link was identified from maladjustment at baseline to rumination 10 weeks later. When the model was tested for males only, the pathways from maladjustment at baseline to stress and perceptions of false self 10 weeks later were no longer significant. These differences could be the result of fluctuations in power across the reduced sample sizes. Overall, it appears that the direction of effect among risk, vulnerability and maladjustment is from maladjustment to risk and vulnerability. 


\section{Discussion}

Gender and Age Trends

As predicted, male and female preadolescents reported similar levels of stress, sense of control, rumination, and maladjustment. However female adolescents reported significantly more stress, rumination, and maladjustment, and less perceived control over their lives than male adolescents. Similar to the results presented in Chapter 2, the effect of gender and age on these constructs was small. Unfortunately, because cell sizes for older males were small and because age was dichotomized, the precise timing of the emergence of gender differences was unable to be tested. Thus, it still remains unclear whether the gender difference in rumination preceded the gender difference in depression, whether the reverse is true, or whether the two gender differences emerge at similar points in time.

Relationships Among Stress, Sense of Control, Rumination, Perceptions of False Self, and Maladjustment.

Vulnerability as moderators between stress and maladjustment. In a replication and extension of Study 2, the vulnerability factors of rumination, sense of control, and perceptions of false self were tested as moderators of the relationship between stress and maladjustment. As predicted, the results of the current study did not support a stress-diathesis model of adolescent depression using longitudinal data, namely, not rumination, sense of control, nor perceptions of false self moderated the relationship between baseline stress and maladjustment at 10 weeks. In combination with the results from Chapter 2, the stress-diathesis model of depression was not supported either concurrently or longitudinally, whether the vulnerability variables were stipulated as rumination, sense of control, or perception of false self. 
Vulnerability as moderators between initial and later maladjustment. A further

hypothesis asserted by RST was tested in the current study. RST asserts that ruminating while depressed will prolong and intensify maladjustment. In this sense, rumination is characterised in RST as a moderator of the relationship between maladjustment at two points in time. In this sample, rumination did not moderate the relationship between baseline maladjustment and maladjustment 10 weeks later. In addition, the vulnerability factors of sense of control and perceptions of false self were also tested as exacerbators of the relationship between initial and later maladjustment. Similarly to rumination, neither sense of control nor perceptions of false self exacerbated maladjustment across time.

Vulnerability as mediators of the relationship between stress and maladjustment. Another goal of this study was to test the mediational model presented in Chapter 2 both concurrently and longitudinally. Concurrently, each previously identified path was confirmed in the current dataset; however, the model did not fit these data very well, based on model fit indices. The model fit indices dramatically improved when maladjustment was allowed to predict rumination and sense of control. This result, in combination with the longitudinal lack of support found in the directions of effect model suggests that direction of effect among these variables differ to the mediational model in Chapter 2. Primarily, the models suggested that an important direction of effect is from maladjustment to risk and vulnerability.

Direction of effect among stress, sense of control, rumination, perceptions of false self and maladjustment across 10 weeks. Interestingly, only baseline maladjustment predicted change in other variables across time. More specifically, maladjustment predicted increases in stress and perceptions of false self, as well as decreases in sense of control 
across time, while initial levels of stress, perception of false self, and sense of control were controlled. This result may indicate that stress, sense of control, and perceptions of false self are not risk factors for maladjustment, but rather are the result of negative feelings. However, an important observation highlights that the stability coefficients in the path models should be taken into account before suggesting that risk and vulnerability factors cannot predict changes in maladjustment across time.

In Figure 5.6, no variables were able to predict rumination or maladjustment across time. Notably, rumination and maladjustment exhibited the highest stability scores. In fact, baseline maladjustment explained $74 \%$ of the variability in maladjustment 10 weeks later, and baseline rumination explained $55 \%$ of the variability of rumination 10 weeks later. Therefore, there was little remaining variance for additional variables to explain. Notably, when the path model was conducted for females only, maladjustment did predict rumination across time, however for females, baseline rumination only predicted $30 \%$ in rumination at 10 weeks, meaning there was more remaining variance to be explained for females. This problem has been noted in previous studies assessing the relationship between rumination and depressive symptoms in adults (Weir et al., 2006). However there is little that can be done to correct this problem. One potential solution is to measure the variables across longer periods of time, as time is likely to increase the opportunity for stability coefficients to reduce. For example, when Weir et al. extended their time period from 5 to 20 weeks, the relationship between rumination and depressive symptoms became bidirectional. Thus, it is unclear whether or not the lack of reciprocal relationships is a genuine effect or if the result is a function of the very high stability coefficient for maladjustment. 
Overall, however, a number of important results should be highlighted from the current study. First, very few differences were found between males and females on the measured variables during preadolescence, however for adolescents, females became somewhat more stressed, ruminative, felt less control over their lives, and reported slightly more maladjustment than males. Second, replicating and extending the results of study 1 , vulnerability did not interact with stress to exacerbate maladjustment across time, nor did vulnerability exacerbate maladjustment across time. Third, the mediational model established in Study 1 was not supported when tested with concurrent or longitudinal data. And last, the direction of effect among risk, vulnerability, and maladjustment appeared to be from maladjustment to risk and vulnerability. 


\section{Chapter 6: The Relationships Among Stress, Rumination, Sense of Control, Perceptions of False Self, and Depressive and Anxious Symptoms}

Study 3 was conducted to overcome three of the major limitations of Study 2

(Chapter 5), as well as replicating and extending upon the previous two studies. The

first major limitation Study 3 addresses is the considerable overlap between depressive and anxious symptoms seen in Study 2. The CDI and the RMCA were so highly

correlated that the two measures were combined into a maladjustment score. As a

result, the comparative predictive ability of stress, perceptions of false self,

rumination, and sense of control could not be tested separately for anxious and

depressive symptoms. To overcome this problem, anxious symptoms are measured in the current study using the Beck Anxiety Inventory (BAI; Beck, Brown, Epstein, \& Steer, 1988). The BAI was used rather than the RMCA because the former scale has less overlap with depressive symptoms (Beck et al., 1988; Jolly et al., 1993).

A more specific measure of anxious symptoms was important because recent research assessing the relationships among rumination, depression and anxiety suggest that the relationship between rumination and depression may be, in part, accounted for by both variables' association with anxious symptoms. For example, Muris, Roelofs et al., (2004) measured rumination, depressive, and anxious symptoms in a sample of 337 adolescents and found that rumination predicted unique variance in depressive symptoms, however when anxious symptoms (worry) were controlled, rumination was no longer related to depressive symptoms. The current study builds on Muris, Roelofs et al.'s research by assessing the interrelationships among rumination, anxiety, and depression across time. 
The second problem the current study aimed to overcome was the high stability of maladjustment. As discussed in Chapter 5, risk and vulnerability factors may not have predicted maladjustment across time because of the lack of remaining variance to be explained in maladjustment after baseline maladjustment was controlled. Possibly 10 weeks was not enough time for sufficient change in maladjustment to occur. Certainly in previous longitudinal studies with adolescents, the time lag has been longer, between 9 months and 2 years (Driscoll, 2004; NolenHoeksema, Stice et al., 2007). Thus, a goal of this study was to extend the period between time points. In the current study, 4 months passed between the two survey administrations, and latent variable modeling was conducted to test the directions of effect among stress, sense of control, rumination, perceptions of false self, and depressive and anxious symptoms. It was predicted that the relationships among risk and vulnerability factors and mood states would be bidirectional; however the direction of effect would be stronger from mood states to risk and vulnerability factors rather than the reverse.

The third problem this study sought to rectify was the inadequate number of adolescent males sampled in Study 2. Because of the small cell sizes for adolescent males, age and gender trends could not be adequately assessed. As a consequence, Study 2 was not able to fully establish whether or not the finding in Study 1-that the gender difference in depression occurred simultaneously with the gender difference in rumination-was a true effect. Thus, a much larger sample size was collected to fully test the gender-based developmental trends of depressive symptoms and rumination at each age level. In line with previous research (Broderick \& Korteland, 2002; Jose \& Brown 2007; Schwartz and Koenig, 1996), females were expected to report 
ruminating more than males, before the age that females begin reporting more depressive symptoms than males.

In addition to overcoming these limitations, the current study extended previous findings in two ways. First, the construct validities of stress, sense of control, rumination, perceptions of false self, and anxious and depressive symptoms were tested. And second, the direction of effect among stress, sense of control, perceptions of false self, rumination, and anxious and depressive symptoms was tested using latent variable modeling ${ }^{36}$. The first issue addresses the question: are stress, sense of control, rumination, and perceptions of false self simply various forms of distress, or are they independent constructs? The latter issue raises the question: which risk, vulnerability, and maladjustment variables predict changes in each other across time?

In the current study the construct validity of stress, sense of control, perceptions of false self, rumination, depressive, and anxious symptoms were assessed using confirmatory factor analysis. So far in this thesis these variables have been assumed to represent independent constructs. However, it is possible that these variables measure the same underlying construct: a general distress factor. The construct validity of these variables requires testing because the interrelationships found in previous chapters and published research could be a function of construct overlap. To date, no published studies have demonstrated divergent validity among these constructs with adolescents, thus relevant studies from the adult literature will be drawn upon.

Gotlib (1984) has argued that the high correlations among different types of maladjustment in sub-clinical populations (for example, between anxious and

\footnotetext{
${ }^{36}$ Moderation models were not assessed because in studies 1 and 2 stress-diathesis models of adolescent maladjustment were not supported.
} 
depressive symptoms) are the result of construct overlap. In other words, Gotlib argues that many different scales actually measure the same thing: general psychological distress. In Gotlib's study, college students completed 17 different scales assessing a range of maladaptive constructs, such as depression, anxiety, dysfunctional attitudes, assertiveness, hostility, and interpersonal sensitivity. Results from exploratory factor analysis suggested there was one predominant factor, which included items from nearly all of the 17 measures ${ }^{37}$. Thus, Gotlib concluded that these supposedly different types of psychopathology actually all represent a general psychological distress construct in sub-clinical populations.

With regards to rumination and depression specifically, it has also been argued that the relationship between rumination and depression may be the result of construct similarity, especially considering the high item similarity between rumination and depression measures (Roberts et al., 1998). To test this hypothesis, Treynor et al. (2003) measured rumination and depression in a general population sample of adults ${ }^{38}$. Results from exploratory factor analyses indicated that rumination and depression items clustered separately in distinct groups, suggesting that rumination and depression are highly related but distinct constructs.

However, there are at least three problems with using exploratory factor analysis to test construct validity. First, exploratory factor analysis does not allow for a priori factors to be stipulated. Second, exploratory factor analysis does not provide fit indices to assess model fit. And last, exploratory factor analysis does not allow error

\footnotetext{
${ }^{37}$ Exploratory factor analysis tests whether scale items cluster together in meaningful ways (Bryant \& Yarnold, 1995). For example, if stress and rumination are separate constructs, then stress and rumination items should cluster in two distinct groups. If however, stress and rumination items are found to mix within clusters, it would suggest that stress and rumination are overlapping constructs. ${ }^{38}$ Twelve items were removed from the RSQ because of content overlap with depression prior to analyses.
} 
terms to correlate. In contrast, confirmatory factor analysis can test and evaluate a priori factor structures with correlated error terms (see Cole, 1987; Marsh \& Hocevar, 1985; Thompson, 2004, for further discussion and comparison of exploratory and confirmatory factor analysis).

The second issue raised in Chapter 5, namely, the direction of effect among the measured variables, was tested using latent variable modeling using a similar approach to that in Chapter 5. Latent variables represent the underlying, unmeasured phenomena of interest-for example, depression-that is approximated using measurement tools such as the CDI. The benefit of latent variable modeling over manifest path modeling is that the latent variables are assumed to be free from random error, which is captured in the manifest or measured variables (Hoyle, 1995). In addition, latent variable modeling enables the reliability of each construct to be estimated, which will be addressed below using a 2-step modeling approach (Anderson \& Gerbing, 1988).

In summary, the current study measured stress, sense of control, rumination, perceptions of false self, and anxious and depressive symptoms across 4 months in a large sample of adolescents. Developmental trends of rumination and depressive symptoms were tested, as well as the construct validity and direction of effect among stress, sense of control, rumination, perceptions of false self, and depressive and anxious symptoms. First, it was predicted that the gender difference in rumination would precede the gender difference in depressive symptoms. Second, it was predicted that stress, sense of control, rumination, perceptions of false self, and depressive and anxious symptoms would be related but distinct constructs. Third, bidirectional relationships were expected among the measured variables-particularly 
between rumination and depression-but that the direction of effect would be stronger from psychopathology to risk and vulnerability factors than the reverse.

\section{Method}

Participants and Procedures

Sixty-two schools throughout the Lower North Island and Nelson Region of New Zealand were sent a letter informing them of the study and given an invitation to participate. These schools were then called for an expression of interest in participation; 11 schools were recruited ${ }^{39}$. Regarding socio-economic status, two schools were decile 6 , three were decile 7 , four schools were decile 8 , one school was decile 9 , and the last school was decile 10. Initially, 1174 participants took part in this study. Two children decided they did not want to participate in the study after hearing the information about the project. A further 34 participants completed less than $95 \%$ of the questionnaire and were deleted from the sample. Thus, the final sample of participants at baseline consisted of 1138 adolescents (623 males, 515 females). There were 254 eleven year olds (111 males, 143 females), 251 twelve year olds (130 males, 121 females), 133 thirteen year olds (88 males, 45 females), 198 fourteen year olds (121 males, 77 females), 120 fifteen year olds (67 males, 53 females), 121 sixteen year olds (75 males, 46 females), and 61 seventeen year olds (31 males, 30 females).

Four months later, 974 participants completed the same survey a second time. Attrition was due predominately to absence at follow-up. Twenty four of these participants were deleted because they completed less than $95 \%$ of the survey, leaving 952 potential matches for the final sample. Twenty six of the phase two participants

\footnotetext{
${ }^{39}$ A total of 2366 consent forms were provided to these 11 schools.
} 
could not be matched to phase one data ${ }^{40}$. Thus, 926 (496 males, 430 females)

participants comprised the final sample. The sample was composed of 202 eleven year

olds (85 males, 117 females), 222 twelve year olds (118 males, 104 females), 109

thirteen year olds (69 males, 40 females), 157 fourteen year olds (91 males, 66

females), 105 fifteen year olds (61 males, 44 females), 93 sixteen year olds (56 males,

37 females), and 38 seventeen year olds (16 males, 22 females). The seventeen year

olds were grouped with the 16 year olds to increase the oldest group's cell size.

Consequently, there were $13216-17$ year olds (72 males, 59 females). The majority

of participants were European New Zealanders (72\%), 12\% were Maori, 2\% were

Asian, 2\% were Pacific Islanders, and 12\% classified their ethnicity as "Other".

The procedure for recruitment was identical to Studies 1 and 2. As in the

previous studies reported here, both parental consent and participant assent were

obtained prior to data collection for participants younger than 16 years. Participants

aged 16 years or older gave their consent to participate-in New Zealand parental

consent is not required for individuals sixteen years and older. Schools received \$2 in

book vouchers for each child who participated during both time points, and each child

received a small token of appreciation (a piece of confectionary/stationary item). In

addition, all participants who took part in both phases of this study were entered into

a prize draw to win either an iPod shuffle, or $\$ 180$ worth of CD vouchers. Some of

the sessions involved larger groups than reported in previous chapters; 10-100

adolescents were surveyed at once. All larger sessions were run in the school hall or

an equally spacious location to ensure privacy for adolescents when completing the survey.

\footnotetext{
${ }^{40}$ Non-matched participants were individuals who had returned a consent form but were either absent at baseline, or who completed less than $95 \%$ of the survey at baseline.
} 
Materials

The measures assessing stress (ELESC; Jose et al., 1994), perceptions of false self (POFS; Chapter 4), sense of control (OSOC; Weir \& Jose, 2006a), rumination (RSQ; Nolen-Hoeksema et al., 1993), and depressive symptoms (CDI; Kovacs, 1992) were identical to those described previously. All previously used measures exhibited high internal reliability in the current sample, at both time points and across all ages (Table 6.1).

Anxiety. The Beck Anxiety Inventory (BAI; Beck et al., 1988) ${ }^{41}$ is a 21 -item measure designed to assess the severity of anxious symptoms in adolescents and adults while minimizing the overlap with depressive symptoms (Beck et al.). Items are scored from $0=$ no symptoms to $3=$ severe symptoms; overall scores range between 0-63. Scores between 19-29 and 29-63 describe anxious symptoms at moderate and severe levels respectively (Beck \& Steer, 1993). In this study, the BAI exhibited excellent internal reliability across all age groups (Table 6.1). Furthermore, in this study the correlation between BAI and CDI scores were not high enough to suggest severe problems with multicollinearity, either at baseline, $r(924)=.64, p<.001$, or at 4 months, $r(924)=.65, p<.001$.

Table 6.1. Scale Reliabilities for Each Age Group at Both Baseline and 4 Months

\begin{tabular}{lcccccc}
\hline & Stress & False Self & Control & Rum & Anxiety & Dep \\
\hline \multicolumn{7}{c}{ Time $1(4$ month follow up) } \\
\hline 11 yrs & $.91(.92)$ & $.80(.84)$ & $.87(.88)$ & $.88(90)$ & $.83(.94)$ & $.91(.90)$ \\
13 yrs & $.90(.91)$ & $.84(.83)$ & $.85(.89)$ & $.90(.90)$ & $.94(.94)$ & $.92(.91)$ \\
14 yrs & $.89(.93)$ & $.80(.87)$ & $.88(.92)$ & $.92(.92)$ & $.93(.95)$ & $.91(.93)$ \\
$15 y r s$ & $.88(.90)$ & $.78(.84)$ & $.88(.92)$ & $.86(.87)$ & $.89(.93)$ & $.87(.91)$ \\
$16 y r s$ & $.89(.89)$ & $.81(.87)$ & $.89(.85)$ & $.90(.88)$ & $.90(.90)$ & $.88(.90)$ \\
Note. Rum = Rumination; Dep $=$ Depression
\end{tabular}

Note. Rum = Rumination; Dep $=$ Depression

\footnotetext{
${ }^{41}$ The Beck Anxiety Inventory is presented in Appendix L
} 


\section{Results}

Analytic Strategy

First, to examine the age and gender trends in this sample on stress, sense of control, perceptions of false self, rumination, and anxious and depressive symptoms, a repeated-measures MANOVA was conducted. Next the concurrent and longitudinal relationships among all variables were examined using zero-order correlations. And last, the construct validity and direction of effect among stress, sense of control, perceptions of false self, rumination, and anxious and depressive symptoms were assessed using a 2-step structural equation modeling approach (Anderson \& Gerbing, 1988). In the first step, measurement models assessed the construct validity of each variable, and then in the second step, structural models tested the directions of effect. Developmental Trends of Stress, False self, Rumination, Sense of Control, and Depressive and Anxious Symptoms.

To explore developmental trends for males and females, a 2 (gender: male, female) x 6 (age: $11,12,13,14,15,16-17$ years) x 2 (time: baseline, follow up) repeated-measures MANOVA was conducted. Age and gender were the betweensubjects factors, time was the within-subjects factor, and stress, rumination, sense of control, perceptions of false self, and depressive and anxious symptoms were the dependent variables.

Multivariate Effects. Within-subjects multivariate effects indicated that there was a significant effect of time, $F(6,909)=10.93, p<.001$, partial $\eta^{2}=.07$. The time $\mathrm{x}$ age interaction reached significance, $F(30,4565)=1.47, p<.05$, partial $\eta^{2}=$ .01 , however because the size of this effect was so small, and because there was no theoretical reason to expect this interaction, univariate effects for this interaction 
were not interpreted. Neither the time $\mathrm{x}$ gender, $F(6,909)=.30, p=.94$, partial $\eta^{2}$ $<.01$, nor the time $\mathrm{x}$ gender $\mathrm{x}$ age interaction, $F(30,4565)=1.13, p=.29$, partial $\eta^{2}=.01$, were significant. Thus, only the univariate effects of time were interpreted. The between-subjects multivariate effects indicated that there was a significant main effect for gender, $F(6,909)=15.74, p<.001$, partial $\eta^{2}=.09$, and a significant main effect for age, $F(30,4565)=2.66, p<.001$, partial $\eta^{2}=.02$. The age $\mathrm{x}$ gender interaction term was marginally significant, $F(30,4565)=1.38, p=$ .08 , partial $\eta^{2}=.01$. All univariate between-subjects effects were interpreted ${ }^{42}$. Univariate Effects. Means and standard deviations for males and females across ages on all variables and at both time points are presented in Table 6.2. Regarding the within-subjects univariate effect of time, stress was found to increase across four months, $F(1,914)=10.75, p<.001$, partial $\eta^{2}=.01$, whereas perceptions of false self, $F(1,914)=34.20, p<.001$, partial $\eta^{2}=.04$, and rumination decreased across 4 months, $F(1,914)=7.39, p<.01$, partial $\eta^{2}=.01$. Notably, the sizes of these significant effects were small. Reported levels of perceived control, and anxious and depressive symptoms did not change significantly across the four month period, $F_{\mathrm{s}}(1$, $914)=.11$ to $1.18, p s=.27$ to .75 , partial $\eta^{2} \leq .01$. Overall these results show there was substantial consistency reported across time in risk, vulnerability, and maladjustment for adolescents aged 11-16 years old.

\footnotetext{
${ }^{42}$ The marginal multivariate interaction was interpreted because of its theoretical relevance.
} 
Table 6.2. Means and Standard Deviations for Adolescents on all Measured Variables.

\begin{tabular}{|c|c|c|c|c|c|c|c|c|c|c|c|c|}
\hline \multicolumn{7}{|c|}{ Time 1} & \multicolumn{6}{|c|}{ Time 2} \\
\hline \multirow[t]{2}{*}{ Yrs } & \multicolumn{2}{|c|}{ Males } & \multicolumn{2}{|c|}{ Females } & \multicolumn{2}{|c|}{ Total } & \multicolumn{2}{|c|}{ Males } & \multicolumn{2}{|c|}{ Females } & \multicolumn{2}{|c|}{ Total } \\
\hline & $\mathrm{M}$ & S.D & $\mathrm{M}$ & S.D. & $\mathrm{M}$ & S.D. & $\mathrm{M}$ & S.D & $\mathrm{M}$ & S.D. & $\mathrm{M}$ & S.D. \\
\hline \multicolumn{13}{|c|}{ Stress } \\
\hline 11 & 17.53 & 16.24 & 14.96 & 13.88 & 16.04 & 14.93 & 18.54 & 17.34 & 15.68 & 14.22 & 16.89 & 15.63 \\
\hline 12 & 12.53 & 11.92 & 15.20 & 14.75 & 13.78 & 13.36 & 12.53 & 12.61 & 14.24 & 13.96 & 13.33 & 13.26 \\
\hline 13 & 13.04 & 10.82 & 18.78 & 16.70 & 15.15 & 13.50 & 16.16 & 16.00 & 20.33 & 16.91 & 17.69 & 16.38 \\
\hline 14 & 17.28 & 13.22 & 19.38 & 13.85 & 18.16 & 13.49 & 16.99 & 13.26 & 22.92 & 15.95 & 19.48 & 14.70 \\
\hline 15 & 12.12 & 9.44 & 19.87 & 15.68 & 15.37 & 12.96 & 14.49 & 10.58 & 22.84 & 16.96 & 17.99 & 13.20 \\
\hline 16 & 14.04 & 9.20 & 20.03 & 11.64 & 16.74 & 10.75 & 15.76 & 11.56 & 20.39 & 10.58 & 17.85 & 11.32 \\
\hline \multicolumn{13}{|c|}{ Perception of false self } \\
\hline 11 & 40.94 & 10.61 & 40.68 & 9.53 & 40.79 & 9.98 & 37.98 & 10.97 & 37.67 & 10.93 & 37.80 & 10.92 \\
\hline 12 & 39.88 & 9.79 & 40.55 & 10.63 & 40.19 & 10.18 & 38.03 & 9.98 & 37.51 & 9.59 & 37.79 & 9.78 \\
\hline 13 & 38.33 & 9.59 & 40.15 & 7.89 & 39.00 & 9.01 & 35.30 & 9.97 & 40.30 & 10.07 & 37.13 & 10.25 \\
\hline 14 & 39.15 & 9.30 & 40.15 & 7.86 & 39.52 & 8.11 & 37.51 & 8.72 & 39.70 & 10.55 & 38.43 & 9.56 \\
\hline 15 & 39.03 & 8.29 & 39.52 & 9.36 & 39.24 & 8.72 & 38.51 & 9.85 & 37.48 & 10.28 & 38.08 & 10.00 \\
\hline 16 & 38.04 & 9.36 & 39.58 & 9.72 & 37.83 & 9.49 & 37.38 & 9.83 & 36.90 & 9.76 & 37.16 & 9.76 \\
\hline \multicolumn{13}{|c|}{ Sense of Control } \\
\hline 11 & 63.71 & 10.30 & 65.91 & 9.83 & 64.98 & 10.06 & 65.98 & 9.49 & 67.68 & 10.38 & 66.96 & 10.03 \\
\hline 12 & 65.42 & 9.06 & 65.40 & 8.97 & 65.41 & 8.99 & 66.09 & 10.23 & 66.86 & 8.79 & 66.45 & 9.57 \\
\hline 13 & 66.26 & 8.73 & 63.35 & 9.78 & 65.19 & 9.20 & 65.83 & 11.01 & 62.70 & 11.06 & 64.68 & 11.08 \\
\hline 14 & 65.81 & 8.94 & 64.30 & 7.94 & 65.18 & 8.54 & 65.20 & 11.19 & 64.74 & 9.16 & 65.01 & 10.35 \\
\hline 15 & 66.56 & 8.80 & 64.75 & 9.16 & 65.80 & 8.95 & 67.02 & 7.27 & 63.59 & 9.87 & 65.58 & 8.58 \\
\hline 16 & 67.10 & 7.91 & 65.73 & 7.28 & 66.48 & 7.63 & 66.93 & 9.41 & 65.27 & 8.68 & 66.18 & 9.09 \\
\hline \multicolumn{13}{|c|}{ Rumination } \\
\hline 11 & 34.44 & 10.06 & 36.89 & 10.53 & 35.86 & 10.38 & 32.76 & 11.17 & 35.28 & 10.49 & 34.22 & 10.83 \\
\hline 12 & 33.77 & 10.10 & 36.55 & 10.08 & 35.07 & 10.16 & 32.25 & 9.71 & 36.31 & 10.25 & 34.15 & 10.15 \\
\hline 13 & 33.80 & 9.71 & 40.90 & 13.61 & 36.40 & 11.75 & 32.54 & 10.24 & 38.90 & 11.55 & 34.87 & 11.12 \\
\hline 14 & 34.15 & 8.46 & 40.29 & 8.80 & 36.73 & 9.10 & 34.85 & 8.63 & 40.65 & 9.72 & 37.29 & 9.52 \\
\hline 15 & 33.48 & 8.59 & 41.46 & 11.66 & 36.82 & 10.70 & 32.46 & 8.15 & 39.87 & 11.15 & 35.56 & 10.16 \\
\hline 16 & 34.88 & 8.87 & 39.17 & 8.78 & 36.81 & 9.05 & 34.58 & 8.50 & 34.49 & 8.04 & 36.80 & 8.62 \\
\hline \multicolumn{13}{|c|}{ Anxiety } \\
\hline 11 & 11.98 & 11.77 & 10.36 & 9.56 & 11.04 & 10.55 & 11.40 & 10.77 & 10.18 & 11.03 & 10.69 & 10.91 \\
\hline 12 & 9.21 & 10.51 & 9.69 & 9.96 & 9.44 & 10.23 & 8.61 & 10.10 & 9.80 & 9.87 & 9.17 & 9.99 \\
\hline 13 & 9.09 & 9.67 & 11.15 & 10.08 & 9.84 & 9.83 & 10.78 & 11.76 & 11.00 & 13.00 & 10.86 & 12.17 \\
\hline 14 & 8.58 & 7.57 & 11.00 & 8.89 & 9.60 & 9.21 & 9.60 & 9.57 & 11.61 & 10.59 & 10.45 & 10.03 \\
\hline 15 & 6.84 & 5.45 & 12.27 & 10.51 & 9.11 & 8.37 & 7.77 & 8.13 & 11.46 & 9.15 & 9.31 & 8.72 \\
\hline 16 & 8.08 & 6.27 & 9.80 & 6.86 & 8.86 & 6.57 & 8.67 & 7.70 & 10.93 & 7.62 & 9.69 & 7.72 \\
\hline \multicolumn{13}{|c|}{ Depression } \\
\hline 11 & 10.66 & 8.97 & 8.49 & 7.67 & 9.40 & 8.29 & 9.57 & 8.01 & 7.66 & 7.94 & 8.46 & 8.00 \\
\hline 12 & 8.46 & 8.85 & 8.17 & 7.74 & 8.32 & 8.33 & 7.76 & 8.24 & 6.62 & 7.17 & 7.22 & 7.76 \\
\hline 13 & 7.72 & 7.86 & 9.20 & 7.83 & 8.27 & 7.84 & 8.55 & 8.34 & 10.73 & 10.42 & 9.35 & 9.17 \\
\hline 14 & 6.85 & 5.21 & 9.73 & 7.61 & 8.06 & 6.47 & 8.07 & 7.86 & 9.70 & 8.64 & 8.75 & 8.00 \\
\hline 15 & 6.66 & 5.13 & 10.02 & 8.00 & 8.07 & 6.67 & 6.82 & 5.74 & 10.50 & 8.71 & 8.36 & 7.33 \\
\hline 16 & 6.47 & 5.64 & 9.10 & 5.48 & 7.66 & 5.70 & 7.32 & 5.81 & 8.98 & 5.74 & 8.07 & 5.81 \\
\hline
\end{tabular}

Univariate between-subjects effects of gender indicated that females reported

higher stress, $F(1,914)=18.01, p<.001$, partial $\eta^{2}=.02$, rumination, $F(1,914)=$

69.93, $p<.001$, partial $\eta^{2}=.07$, anxious symptoms, $F(1,914)=6.51, p<.01$, 
partial $\eta^{2}=.05$, and depressive symptoms, $F(1,914)=5.65, p<.05$, partial $\eta^{2}=$ .01. There were no significant differences between males and females on perceptions of false self, $F(1,914)=1.15, p=.28$, partial $\eta^{2}<.01$, or perceived control, $F(1$, $914)=2.66, p=.10$, partial $\eta^{2}<.01$.

Univariate between-subjects effects for age were significant for stress, $F(5$, $914)=4.14, p<.01$, partial $\eta^{2}=.02$, and rumination, $F(5,914)=3.05, p<.01$, partial $\eta^{2}=.02$, however none of the post hoc tests (employing Bonferroni corrections) for age were significant on rumination. The only significant post hoc test for stress showed that 14 year olds $(M=19.12, S E=1.00)$ were significantly more stressed than 12 year olds $(M=13.63, S E=.83)$.

The univariate age $\mathrm{x}$ gender interactions were not significant for sense of control, perceptions of false self, rumination, or anxiety, $F_{\mathrm{s}}(5,914)=.86$ to $4.86, p \mathrm{~s}$ $=.10$ to .51 , partial $\eta^{2} \leq .01$. The univariate age $\mathrm{x}$ gender interactions were significant for stress, $F(5,914)=3.43, p<.01$, partial $\eta^{2}=.02$, and depressive symptoms, $F(5,914)=3.51, p<.01$, partial $\eta^{2}=.02$. To probe these interactions, a 2 (gender: male, female) x 2 (time: baseline, follow up) repeated measures MANOVA was conducted on stress and depressive symptoms, split by age ${ }^{43}$. Regarding the age $\mathrm{x}$ gender interaction of stress (Figure 6.1), males and females reported similar levels of stress at 11 years old, $F(1,200)=1.87, p=.17$, partial $\eta^{2}$ $=.01$, and 12 years old, $F(1,220)=1.77, p=.19$, partial $\eta^{2}=.01$. However females reported more stress than males at 13 years old, $F(1,107)=3.96, p=.04$, partial $\eta^{2}=.04,14$ years old-marginally, $F(1,155)=3.68, p=.06$, partial $\eta^{2}=$

\footnotetext{
${ }^{43}$ Post hoc $t$ tests were not conducted because of the repeated measures design of the current study. Specifically, post hoc $t$ tests would not be able to test for gender differences at each age group using the composite scores of stress and depressive symptoms across time.
} 
$.02,15$ years old, $F(1,103)=12.96, p<.001$, partial $\eta^{2}=.11$, and 16 years old, $F(1,129)=10.82, p<.001, \operatorname{partial} \eta^{2}=.08$.

Regarding the age $\mathrm{x}$ gender interaction for depressive symptoms (Figure 6.2), males reported marginally more depressive symptoms than females at 11 years old, $F(1,200)=3.61, p=.06$, partial $\eta^{2}=.02$. Males and females reported similar levels of depressive symptoms at 12 years old, $F(1,220)=.48, p=.49$, partial $\eta^{2}=.01$, and 13 years old, $F(1,107)=1.40, p=.24$, partial $\eta^{2}=.01$. However females reported more depressive symptoms than males at 14 years old, $F(1,155)=4.22, p<$ .05 , partial $\eta^{2}=.03,15$ years old, $F(1,103)=7.58, p<.01$, partial $\eta^{2}=.07$, and 16 years old, $F(1,129)=5.53, p<.05$, partial $\eta^{2}=.04$.

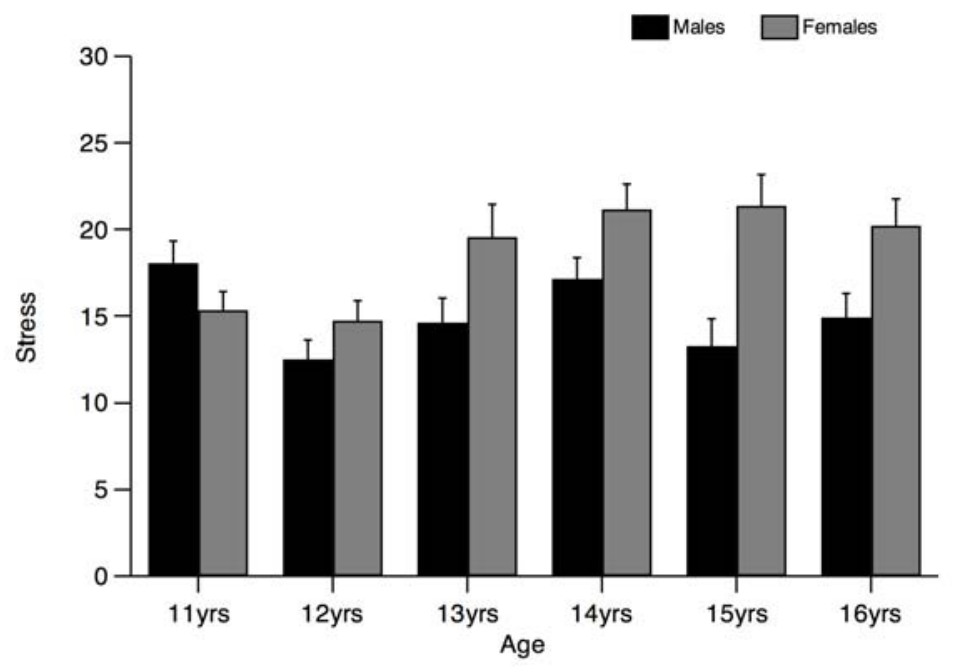

Figure 6.1. Reported rates of stress by males and females aged 11-16 yrs 


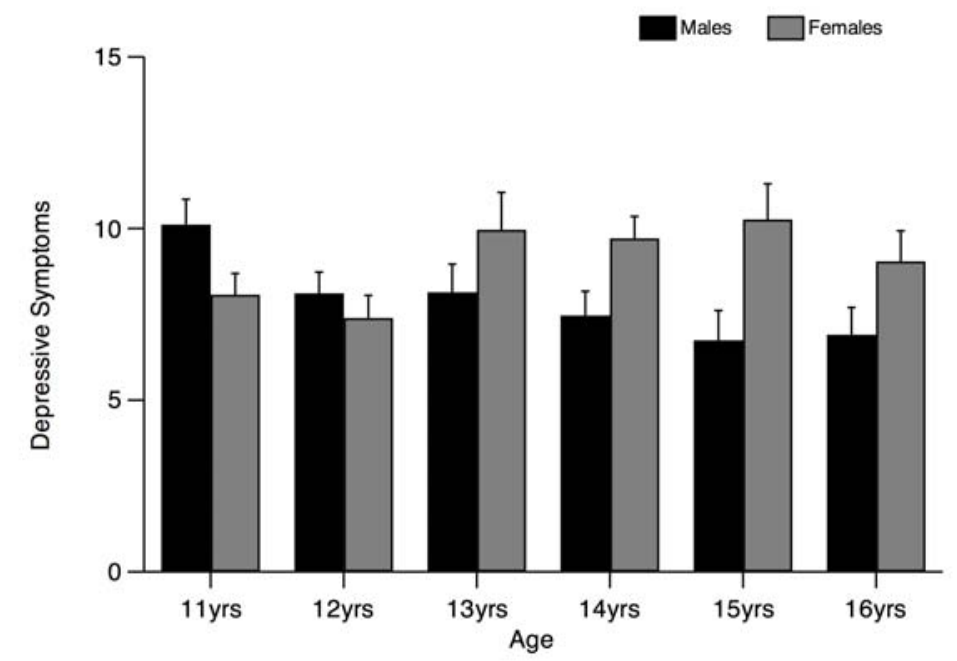

Figure 6.2. Reported rates of depressive symptoms by males and females aged 11-16 yrs

One of the goals of the current study was to examine whether or not the gender difference in rumination preceded the development of the gender difference in depressive symptoms. Recall that females reported ruminating more than males overall in this sample. Although the age by gender interaction was not significant, the gender difference at each age group was probed to test the ages at which females reported higher levels of rumination than males. A 2 (gender: male, female) x 2 (time: baseline, follow up) ANOVA on rumination scores was conducted, split by age. Figure 6.3 illustrates the levels of rumination for males and females aged 11-16 years. Specifically, females reported marginally more rumination than males at 11 years old, $F(1,200)=3.42, p=.07$, partial $\eta^{2}=.02$, However, females reported ruminating more than males at 12 years old, $F(1,220)=4.97, p<.01$, partial $\eta^{2}=$ $.04,13$ years old, $F(1,107)=12.27, p<.001$, partial $\eta^{2}=.10,14$ years old, $F(1$, $155)=21.00, p<.001$, partial $\eta^{2}=.12,15$ years old, $F(1,103)=19.01, p<.001$, partial $\eta^{2}=.16$, and 16 years old, $F(1,129)=11.28, p<.001$, partial $\eta^{2}=.08$. 


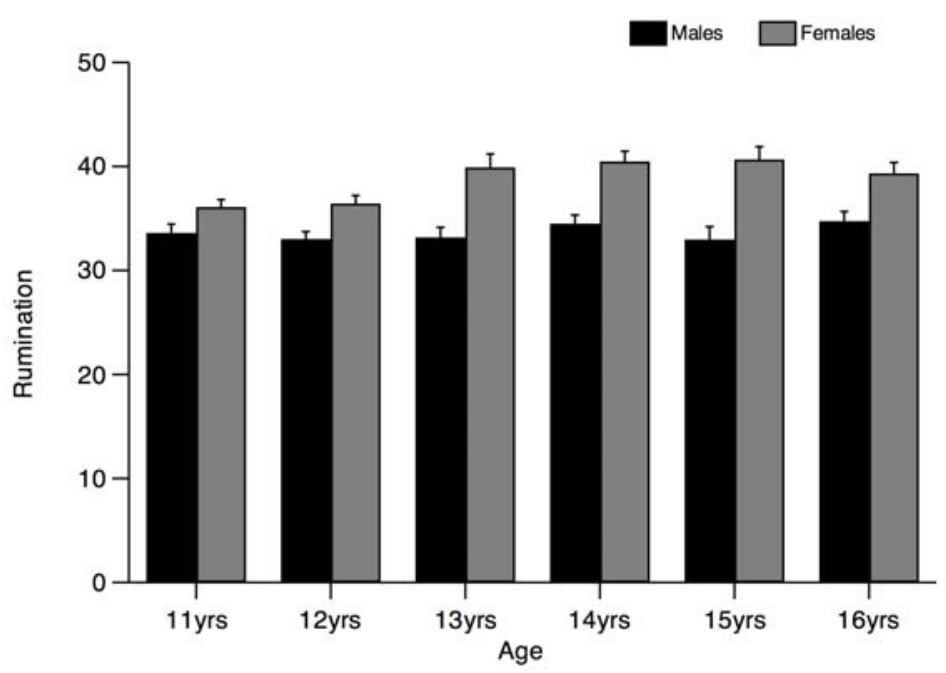

Figure 6.3. Reported rates of rumination by males and females aged 11-16 yrs

Therefore, females began ruminating more than males from age 12, two years before the gender difference in depressive symptoms arose. However, similar to the results of Studies 1 and 2, the sizes of effects of age and gender on all measured variables were small. Therefore, the significant differences between males and females and across age groups should be interpreted in light of the particularly large sample size of the current study.

Relationships Among Stress, False Self, Sense of Control, Rumination, Anxious, and Depressive Symptoms

Correlations. Concurrent and longitudinal correlations were conducted to replicate the interrelationships among stress, false self, sense of control, rumination, and depressive symptoms reported in Chapter 5. Consistent with previous findings, all variables were concurrently intercorrelated at both baseline and 4 months in the expected directions (Table 6.3). In all cases the strength of association was moderate to strong (Cohen, 1992). Regarding the relationships among risk and vulnerability factors and anxious symptoms, at both time points higher stress, false self perceptions, and rumination, and lower perceived control were related to more anxious 
symptoms. Again, the strength of association among these variables was moderate to strong and in the expected direction.

Table 6.3. Concurrent Correlations Among Stress, False Self Perceptions, Sense of Control, Rumination, Anxiety, and Depression.

\begin{tabular}{lcccccc}
\hline & Stress & False Self & Control & Rumination & Anxiety & Depression \\
\hline Stress & - & $.40 * * *$ & $-.50 * * *$ & $.48 * * *$ & $.55 * * *$ & $.63 * * *$ \\
False Self & $.48 * * *$ & - & $-.54 * * *$ & $.44 * * *$ & $.43 * * *$ & $.52 * * *$ \\
Control & $-.52 * * *$ & $-.57 * * *$ & - & $-.45 * * *$ & $-.45 * * *$ & $-.64 * * *$ \\
Rumination & $.54 * * *$ & $.48 * * *$ & $-.45 * * *$ & - & $.54 * * *$ & $.54 * * *$ \\
Anxiety & $.60 * * *$ & $.45 * * *$ & $-.50 * * *$ & $.54 * * *$ & - & $.64 * * *$ \\
Depression & $.67 * * *$ & $.57 * * *$ & $-.69 * * *$ & $.55 * * *$ & $.65 * * *$ & - \\
\hline
\end{tabular}

Note: Time one correlations are above the diagonal, Time two correlations are below the diagonal. $* * * p<.001$. All significance tests were one-tailed.

Also consistent with the findings presented in Chapter 5, all variables were longitudinally correlated with one another in the expected directions (Table 6.4). However the intercorrelations were somewhat smaller than those presented in Chapter 5. Thus, as expected, the relationships among stress, false self, sense of control, rumination, and anxious and depressive symptoms were replicated across 4 months, however, as expected, the stability of each construct was slightly reduced compared to the 10 week data.

Table 6.4. Longitudinal Relationships Among Stress, False Self Perceptions, Sense of Control, Rumination, Anxiety, and Depression.

\begin{tabular}{lcccccc}
\hline & Stress T1 & False T1 & Con T1 & Rum T1 & Anx T1 & Dep T1 \\
\hline Stress T2 & $.64 * * *$ & & & & \\
False T2 & $.35 * * *$ & $.66 * * *$ & & & \\
Con T2 & $-.43 * * *$ & $-.46 * * *$ & $.65 * * *$ & & \\
Rum T2 & $.41 * * *$ & $.32 * * *$ & $-.33 * * *$ & $.64 * * *$ & & \\
Anx T2 & $.42 * * *$ & $.34 * * *$ & $-.37 * * *$ & $.41 * * *$ & $.65 * * *$ & \\
Dep T2 & $.55 * * *$ & $.45 * * *$ & $-.54 * * *$ & $.48 * * *$ & $.56 * * *$ & $.75 * * *$ \\
\hline \multicolumn{7}{l}{ Note: T2 } \\
$<.001$. All significance tests were one-tailed.
\end{tabular}


Construct Validity and the Direction of Effect among Risk Factors and Maladjustment

A two-step modeling approach (Anderson \& Gerbing, 1988; Kline 2005) was adopted to examine the construct validity and direction of effect among the variables. In a two-step approach, the measurement model is tested for appropriate fit (using confirmatory factor analysis) prior to the structural model. This ensures that the structural model is based on an appropriate measurement model. For the purposes of this study, the measurement model allows a stringent test of the construct validity of measured variables by examining both criterion and discriminant validity. Criterion validity tests whether the individual indicators of each latent variable are valid.

Discriminant validity tests whether the manifest and latent variables are distinct across constructs.

Measurement Models. Two measurement models were tested prior to testing the structural model. The first measurement model included baseline variables; the second included the variables at 4 months. For each model, items for each scale were reduced to three parcels. Parcels were created because of the large number of items used in the current scales. Creating parcels enabled the number of indicators (and corresponding number of error terms) to be reduced, thus reducing the complexity of the model. By reducing the complexity of the model, the likelihood of spurious correlations is reduced (Little et al., 2002). In addition, Little et al. have noted that parcelled items may be more appropriate because single items often share variance which is not of interest, or are less reliable than composite indicators ${ }^{44}$. For example, if parcels were not created in this study, the latent variable of stress would have had

\footnotetext{
${ }^{44}$ It should also be noted that not all authors agree that parcels are an appropriate way to specify latent variables. An in-depth discussion of these issues is beyond the scope of this thesis (see Little et al., 2002 for more information).
} 
40 indicators; the false self latent variable would have had 16 indicators, and so on. The parcels were created identically for baseline and time 2 variables.

Specifically, for the stress scale, parcel one included items 1-13, parcel two included items 14-26, and parcel three included items 27-40. For the false self scale, parcel one included items 1-6, parcel two included items 7-11, and parcel three included items 12-16. For the sense of control scale, parcel one included items 1-6, parcel two included items 7-12, and parcel three included items 13-17. For the rumination scale, parcel one included items 1-6, parcel two included items 7-12, and parcel three included items 12-16. For the anxiety scale, parcel one included items 17, parcel two included items 8-14, and parcel three included items 15-21. For the depression scale, parcel one included items 1-9, parcel two included items 10-18, and parcel three included items 19-27. For each construct, parcels exhibited high internal reliability at both baseline ( $\alpha \mathrm{s}=.81$ to .90$)$ and 4 month follow up ( $\alpha \mathrm{s}=.80$ to .91$)$.

Six latent variables were created reflecting stress, perceptions of false self, sense of control, rumination, and anxious and depressive symptoms. The path from the latent variable to its first indicator was set to 1 , to set the metric scale for each manifest variable (Kline, 2005). As is common in CFA, the latent variables were allowed to correlate with each other. Results from the baseline measurement model indicated that each parcel loaded highly on its corresponding latent variable (Figure 6.4). This model fit these data well, apart from the chi-square ratio statistic, $\chi^{2} / d f=$ 4.07, GFI $=.94, A G F I=.91, \mathrm{sRMR}=.04, \mathrm{RMSEA}=.06$. Thus, the measurement model fit these data quite well at baseline. 


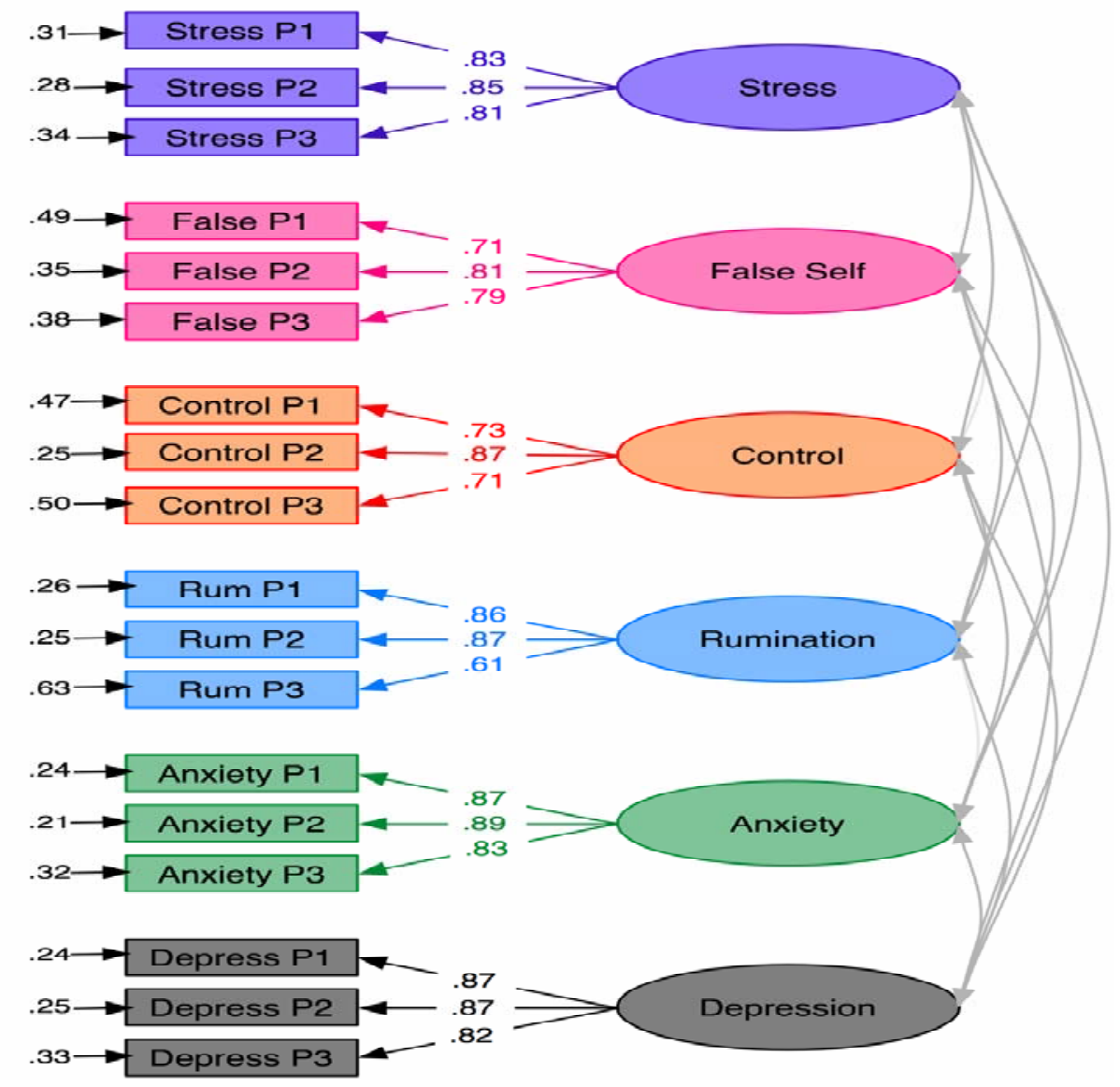

Figure 6.4. Measurement model for baseline variables

This measurement model was also tested using the 4-month data; all indicators loaded highly on its corresponding latent variable $(.57-.91)$. Again, the model fit these data well, except for the chi-square ratio statistic, $\chi^{2} / d f=3.97, G F I=$ $.94, A G F I=.92, \mathrm{sRMR}=.04, \mathrm{RMSEA}=.06$. Therefore, at both time points, the constructs exhibited adequate criterion validity. The correlations among the latent variables for both time points were mainly moderate in strength (Table 6.5), suggesting that the six latent variables are related constructs. However, the correlations among the latent variables do not suggest that risk, vulnerability, and maladjustment variables all represent the same construct, namely, general psychological distress, as suggested by Gotlib (1984). In essence, the overall moderate 
strength of correlations suggest that stress, false self, sense of control, rumination, anxiety, and depression are related, but independent constructs.

Table 6.5. Correlations Among Latent Variables for the Baseline and 4 Month Measurement Models.

\begin{tabular}{lcllccc}
\hline & Stress & False & Control & Rumination & Anxiety & Depression \\
\hline Stress & & $.48 * * *$ & $-.61 * * *$ & $.57 * * *$ & $.62 * * *$ & $.72 * * *$ \\
False & $.56 * * *$ & & $-.65 * * *$ & $.57 * * *$ & $.51 * * *$ & $.61 * * *$ \\
Control & $-.60 * * *$ & $-.67 * * *$ & & $-.58 * * *$ & $-.53 * * *$ & $-.75 * * *$ \\
Rumination & $.63 * * *$ & $.60 * * *$ & $-.57 * * *$ & & $.62 * * *$ & $.64 * * *$ \\
Anxiety & $.66 * * *$ & $.51 * * *$ & $-.58 * * *$ & $.62 * * *$ & & $.72 * * *$ \\
Depression & $.76 * * *$ & $.66 * * *$ & $-.80 * * *$ & $.64 * * *$ & $.72 * * *$ & \\
\hline
\end{tabular}

Note. Values above the diagonal represent baseline correlations; values below the diagonal represent time two correlations. $*_{*}^{*}<<.001$. All significance tests were one-tailed.

Structural Models. Because the measurement models fit these data adequately, structural models were conducted next to test directions of effect. The models were stipulated in a similar fashion to Chapter 5 . That is, an exploratory approach was adopted where initially all path stabilities and cross-lag paths were included in the models, and then non-significant paths were pruned from the model (Kline, 2005).

An exploratory approach was adopted for two reasons. First, the current study's sample size and longer timeframe were expected to enable additional significant cross lag paths to be identified. And second, different relationships might exist between risk factors and mood states when depressive and anxious symptoms are considered separately.

Similar to Chapter 5, initially a cross lag model was conducted with rumination and depressive symptoms only, in an attempt to replicate the bidirectional relationship seen between rumination and depressive symptoms by Nolen-Hoeksema, Stice et al., (2007). The cross lag model included four latent variables: rumination at baseline, rumination at follow up, depression at baseline, and depression at follow up. Latent variables were allowed to correlate concurrently with each other at both baseline and at 4 months because the measurement models indicated that latent 
variables were all significantly correlated. Each latent variable was measured using three parcels, as described above. The autocorrelations between each manifest indicator over time were allowed to correlate. For example, the first parcel for rumination at baseline was allowed to correlate with the first parcel for rumination at follow up ${ }^{45}$. Regarding the structural pathways, 2 stability pathways (one for each latent construct) were allowed to be estimated. In addition, rumination at baseline was allowed to predict depression at 4 months, and baseline depression was allowed to predict rumination at 4 months. Therefore this initial model was fully saturated. As can be seen from Figure 6.5, both rumination and depression were stable across time. There was partial support for a bidirectional relationship between rumination and depression. Specifically, depression predicted increases in rumination across 4 months, and rumination marginally $(p=.06)$ predicted increases in depressive symptoms across 4 months. Model fit indices were not obtained because the model was fully saturated.

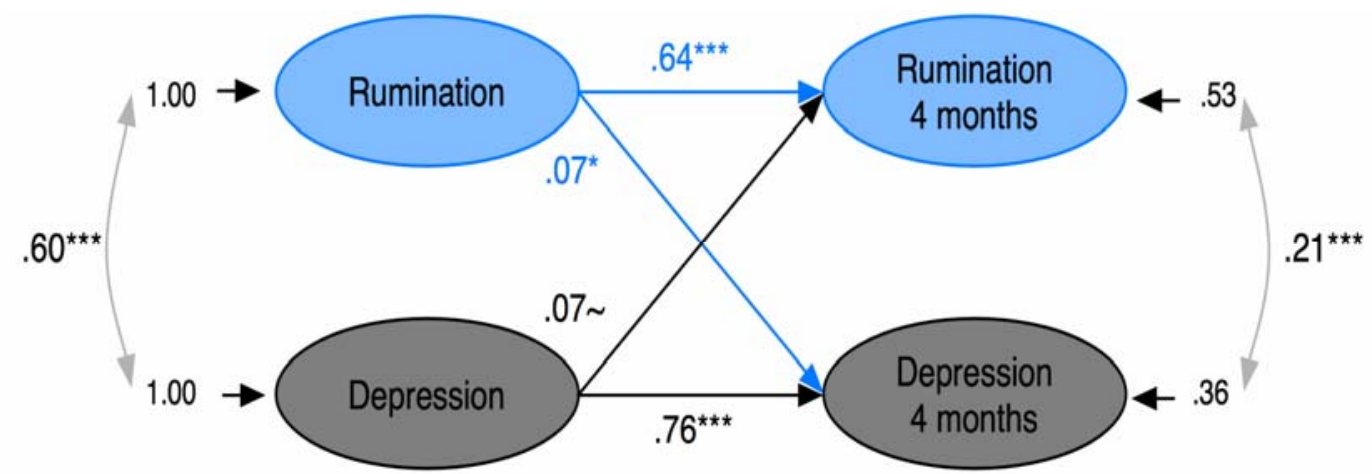

Figure 6.5.Direction of effect between rumination and depression across 4 months

Next, a direction of effect model was conducted involving stress, sense of control, and perceptions of false self in an attempt to replicate the finding presented in Chapter 5-that maladjustment has a larger impact on risk and vulnerability than the

\footnotetext{
${ }^{45}$ Neither autocorrelations nor parcels are represented in figures for ease of interpretation.
} 
reverse. Anxiety was not included in this model initially to establish the direction of effect among risk, vulnerability, and depressive symptoms first. This decision was made based on the finding cited earlier (Muris, Roelofs, et al., 2004) that the relationship between vulnerability and depressive symptoms substantially reduces when anxiety is included.

The initial exploratory model included 10 latent variables (stress, false self, sense of control, rumination, and depressive symptoms at both time points). Similar to the cross-lag model for rumination and depression, all latent variables were allowed to correlate concurrently at both baseline and follow up, each latent variable was measured using the three parcels described above, and the autocorrelations between each manifest indicator over time were correlated. Therefore, there were 30 manifest indicator variables included in the model (15 manifest indicator variables for each time point). Regarding the structural pathways, 5 stability pathways (one for each latent construct) were estimated. Each baseline latent variable was also allowed to predict the remaining 4 latent variables at follow up. In total, therefore, there were 25 structural pathways stipulated.

Results of this base model indicated that all stability coefficients were significant, $\beta \mathrm{s}=.54$ to $.66, \mathrm{ps}_{\mathrm{s}}<.001$. The following four cross-lag paths were significant: baseline stress to later rumination $(\beta=.10, p<.05)$, baseline perceptions of false self to later sense of control, $(\beta=-.09, p<.05)$, baseline rumination to later stress, $(\beta=.14, p<.01)$, and baseline depressive symptoms to later sense of control, $(\beta=-.17, p<.01)$. The cross-lag path from baseline depressive symptoms to false self, $(\beta=.12, p=.06)$ was marginal, and the path from baseline depressive symptoms to stress, $(\beta=.10, p=.10)$, non-significant. This unpruned model fit these data well, 
$\chi^{2} / d f=2.85, G F I=.93, A G F I=.91, \mathrm{sRMR}=.04, R M S E A=.05$. Next, the model was pruned in an identical fashion to that described in Chapter 5. To reiterate, each non-significant path was removed individually, from the least significant to the most. At each stage of the model pruning process, fluctuations in beta weights, phis, and error terms were assessed to ensure multicollinearity was not a large problem.

The pruning process took 11 steps $^{46}$, and two new paths were identified in this process (Figure 6.6). The new paths were from baseline stress to later depressive symptoms and from baseline sense of control to later depressive symptoms. The previously marginal paths from baseline depressive symptoms to later stress and perceptions of false self also became significant. This pruned model fit these data well, $\chi^{2} / \mathrm{d} f=2.77, G F I=.93, A G F I=.91, \mathrm{sRMR}=.04, \mathrm{RMSEA}=.05$.

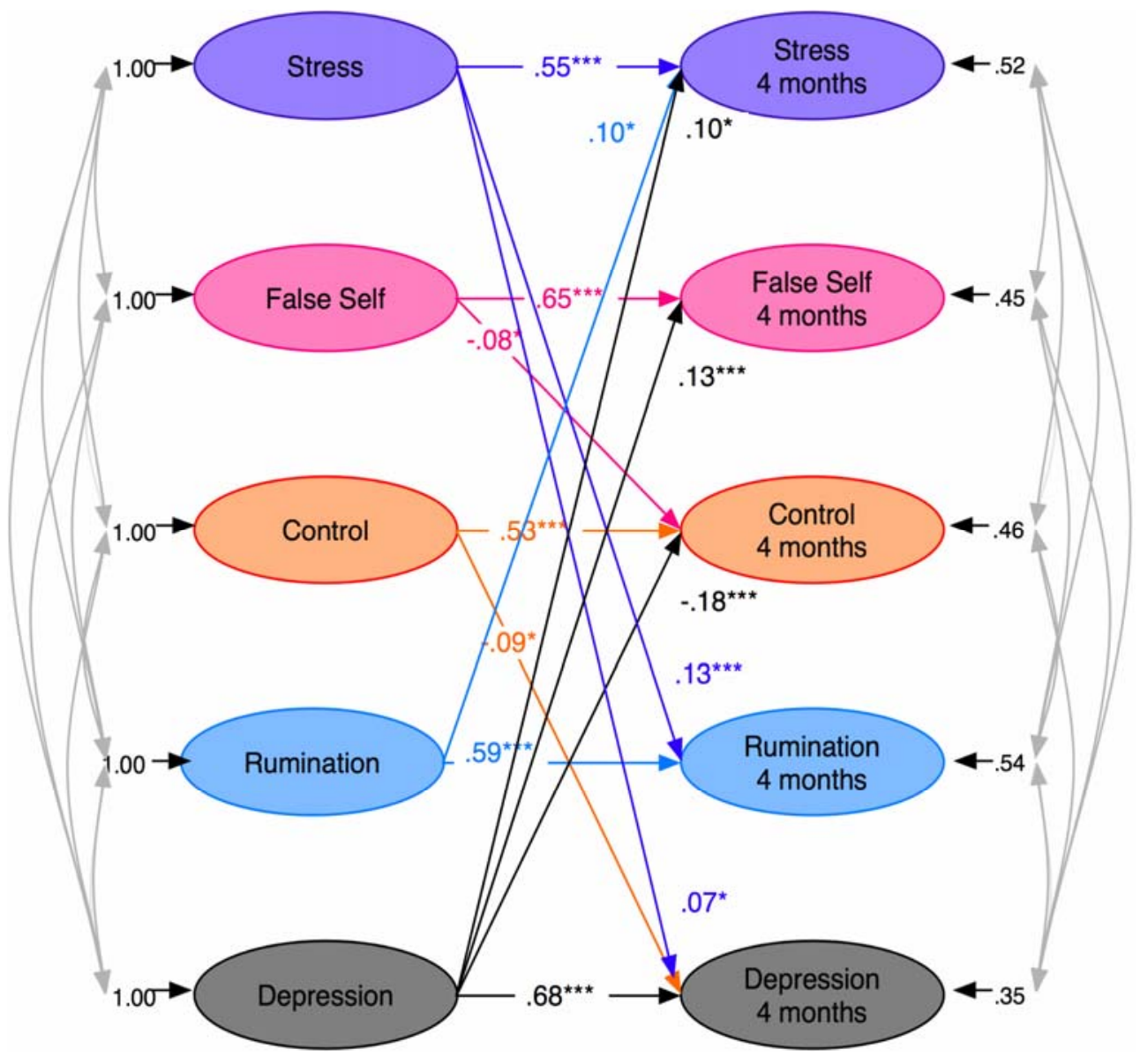

${ }^{46}$ At one step, coefficients had the same Z-score and were removed in unison 
Figure 6.6. Pruned direction of effect model without anxious symptoms

Therefore, reciprocal relationships were observed between stress and depressive symptoms, between sense of control and depressive symptoms, and between stress and rumination. In addition, depressive symptoms predicted stress, sense of control, and perceptions of false self. Interestingly, when these additional risk and vulnerability factors were included in the model, the initial bidirectional relationship between rumination and depression identified above disappeared.

Next, anxious symptoms were included in the model to explore whether anxiety influences the relationships among, risk, vulnerability, and depressive symptoms. Again, the model was initially run as an exploratory base model where all latent variables were allowed to predict all other latent variables at follow up. This base model fit these data well, $\chi^{2} / d f=2.52, G F I=.92, A G F I=.90, \mathrm{~s} R M R=.04$, $R M S E A=.04$, and 10 of the 36 paths were significant. Specifically, all six stability paths were significant, $\beta \mathrm{s}=.49$ to $.63, \mathrm{ps}<.001$. The following cross-lag paths were significant: anxious symptoms at baseline predicted later stress $(\beta=.26, p<.001)$, false self $(\beta=.11, p<.05)$, rumination $(\beta=.14, p<.01)$, and depressive symptoms $(\beta=.09, p<.05)$. In addition, baseline depressive symptoms marginally predicted decreases in sense of control across 4 months $(\beta=-.13, p=.059)$.

In total, the pruning process took 18 steps $^{47}$. This process identified 4 additional significant pathways, resulting in a total of 15 identified cross lag paths (see Figure 6.7). First, baseline perceptions of false self predicted decreases in later perceived control. Second, higher baseline perceived control predicted later decreases in both anxiety and depression. And third, higher baseline anxiety predicted decreases

\footnotetext{
${ }^{47}$ At three steps, coefficients had the same Z-score and were removed in unison.
} 
in later perceived control. Thus, all of the newly identified paths included the construct of perceived control. This pruned model fit these data well, $\chi^{2} / d f=2.46$, $G F I=.92, A G F I=.90, \mathrm{sRMR}=.04, \mathrm{RMSEA}=.04$.

Next, this pruned model including all measured variables was tested using younger (11-13 years) and older adolescents' (14-16 years) data separately, as well as for males and females. This was done to test whether the interrelationships among risk, vulnerability and mood states differed according to the age and gender of adolescents. Overall, the patterns of results were similar across age and gender; anxiety had the largest effect in the model, and although some paths became marginally significant in some of the models ${ }^{48}$, these models were not interpreted because the fit indices fell well below acceptable standards on a number of fit indices. Summary of direction of effect models. When rumination and depression were measured in isolation, partial support was found for a bidirectional relationship. Specifically, rumination marginally predicted increases in depression, whereas depression significantly predicted increases in rumination across 4 months. In the second model, when additional risk and vulnerability factors were included, a number of bidirectional relationships were identified-between stress and depression, between stress and rumination, and between sense of control and depression. Although these bidirectional relationships were identified, the direction of effect was stronger from depressive symptoms to risk and vulnerability rather than the reverse. Interestingly, when additional variables were included in this model, the bidirectional relationship between rumination and depression was entirely eliminated. By including anxiety in the mix, an interesting trend emerged-all bidirectional relationships were eliminated

\footnotetext{
${ }^{48}$ Non-significant paths were the least powerful paths in the overall pruned model, e.g. the link from baseline perceptions of false self to 4 month perceived control was not significant for younger adolescents and males
} 
except for sense of control and maladjustment, and the direction of effect became predominantly from anxiety to risk, vulnerability, and depressive symptoms. In combination, these results suggest that a specific type of maladjustment-anxiety-is the predominant predictor of risk, vulnerability, and maladjustment during adolescence.

And second, measuring additional variables (i.e., so-called "third variables") beyond rumination and depression reduces the impact that rumination and depression have on each other.

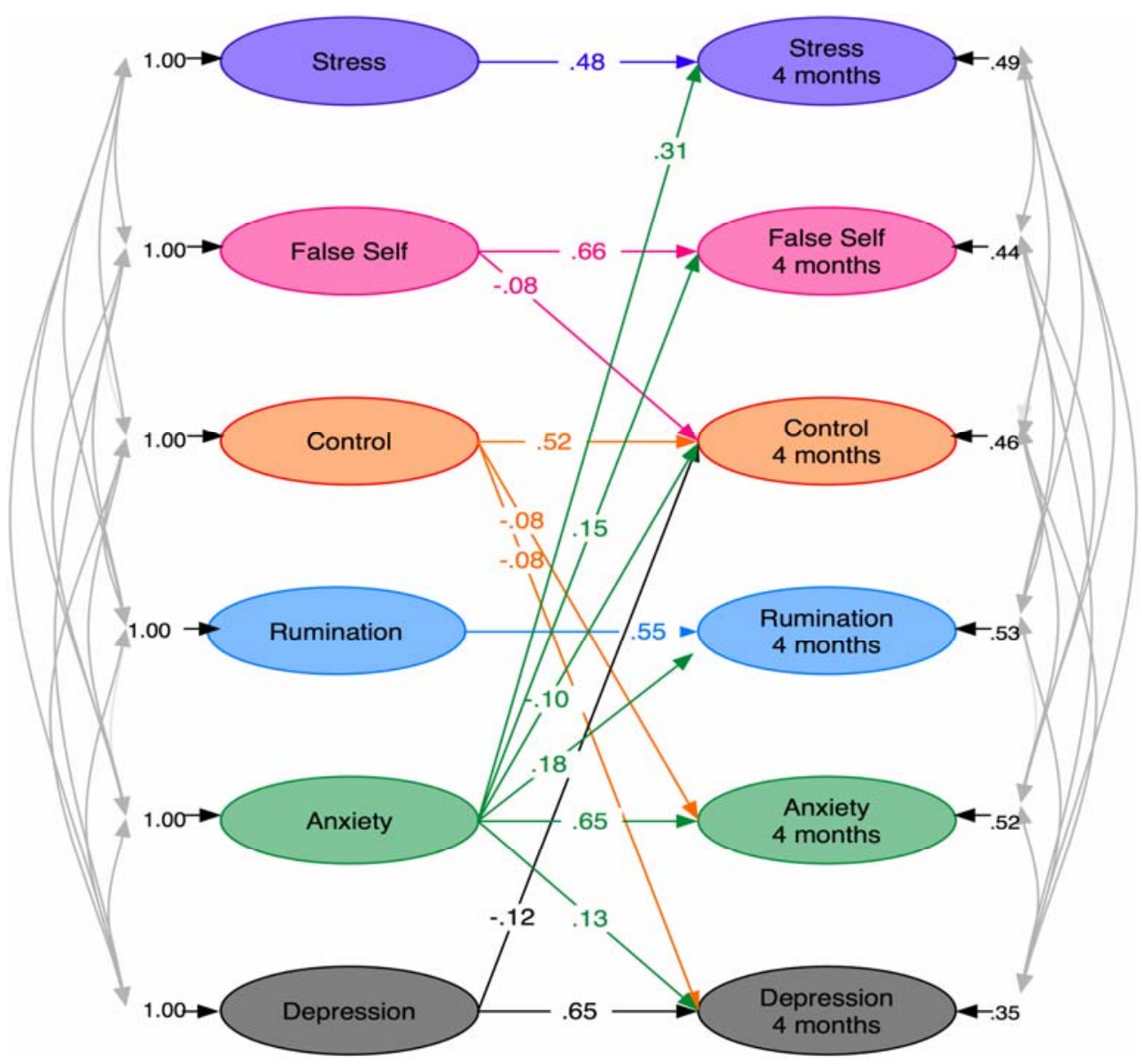

Figure 6.7. Direction of effect model with anxiety included for the total sample 


\section{Discussion}

The goals of study 3 were to examine the developmental trends of risk, vulnerability, and maladjustment in adolescence, to test the construct validity of the measured variables, and to explore the direction of effect among all variables. To achieve these goals, a large sample of adolescents was recruited, and their levels of stress, sense of control, perceptions of false self, rumination, and anxious and depressive symptoms were measured twice, across 4 months.

Developmental trends showed that stress and rumination were more prevalent among females prior to the development of the gender difference in depressive symptoms. Recall that females reported ruminating more than males from 12 years old, females reported higher stress than males from 13 years old, but did not report more depressive symptoms than males until 14 years old. Taken together, these results support the original predictions of Nolen-Hoeksema and Girgus (1994) in that females report higher stress and rumination than males, and also begin reporting more depressive symptoms than males from early adolescence. Although these differences were statistically significant, as seen throughout this thesis, the sizes of these effects were small and are traceable, at least in part, to the particularly large sample sizes.

However, it is not likely that these gender differences in stress and rumination are prompting the gender difference in depressive symptoms because the direction of effects model showed that neither stress nor rumination predicted change in depressive symptoms across time. For RST to be supported, two predictions required substantiation. First, females need to report higher risk and vulnerability for maladjustment prior to the development of gender differences in maladjustment. 
Second, risk and vulnerability factors are required to predict changes in maladjustment across time. Only the first prediction was met in this current study. These results suggest that even though females reported slightly more risk and vulnerability, the risk and vulnerability states did not actually predict later maladjustment.

It was predicted that there would be bi-directional relationships between risk and vulnerability, and maladjustment (Nolen-Hoeksema, Stice et al., 2007; Weir et al. 2006); however, only partial support was gained for the bidirectional hypothesis. Regarding rumination and depression, when these variables were examined in isolation-as has been done in previous research (Nolen-Hoeksema, Stice et al; Weir et al.)-there was partial support found in the present dataset for a bidirectional relationship, supporting RST. When additional risk and vulnerability factors were included in the model, additional bidirectional models were identified-between stress and depression, between stress and rumination, and between sense of control and depressive symptoms. Interestingly, the bidirectional relationship between rumination and depression was eliminated when additional variables were entered into the model. A last direction of effects model was stipulated which also included anxiety, and interestingly, in this last model, the bidirectional relationships that involved stress were then eliminated from the model. In the final model anxiety predicted all other variables, and was the dominant construct influencing the wellbeing of adolescents regardless of their age or gender. By comparison, depressive symptoms only predicted decreases of sense of control across the 4 month period. Therefore, by measuring anxious and depressive symptoms separately, this study showed that anxious symptoms rather than depressive symptoms predicted change in risk and vulnerability. Although RST has recently been extended to incorporate additional negative states 
such as anxiety (Nolen-Hoeksema, 2000; Nolen-Hoeksema, Wisco et al., 2007), the predominant focus to date has been on depressive symptoms. Certainly, RST would not have predicted a specific unidirectional relationship between rumination and anxiety as seen here, where anxious symptoms predicted increases in rumination across 4 months.

In one of the only previous studies which has assessed the interrelationships between rumination, depression and anxiety, Muris, Roelofs et al. (2002), found that the concurrent relationship between rumination and depressive symptoms was reduced to non-significance when anxiety was controlled. The present study supported Muris, Roelofs et al.’s findings longitudinally by showing that when rumination and depression were considered in isolation there was partial support for a bidirectional relationship, however as soon as additional variables were included in the model, the relationship between rumination and depression disappeared. These results suggest that when rumination and depressive symptoms are measured in isolation of additional variables, spurious paths may become identified. In summary, the direction of effect models show that the effect of maladjustment on risk and vulnerability is stronger than the reverse, and furthermore, that anxious symptoms may be the driving force behind the interrelationships among risk, vulnerability, and depressive symptoms during adolescence. Regarding RST, it appears that the relationship between rumination and depression may be "explained" by a third variable-anxiety.

Of course, these relationships are only meaningful if they are not the function of construct overlap, a possibility raised by Gotlib (1984). In the current study, confirmatory factor analysis was used to test the construct validity of stress, sense of control, rumination, perceptions of false self, and anxious and depressive symptoms. 
Recall that Gotlib argued that the interrelationships among different types of maladjustment in sub-clinical populations are the function of each scale tapping the same underlying construct: general psychological distress. In the current study, using a traditional confirmatory factor analysis, it was found that yes, all of the latent variables were significantly correlated, however not highly enough to suggest that each variable represents the same construct.

In summary, study 3 showed that even though the gender difference in stress and rumination emerged prior to the development of the gender difference in depressive symptoms, neither stress nor rumination reliably predicted change in maladjustment across time. Contrary to what RST would predict, the specific state that predicted changes in depressive symptoms was anxiety, not rumination. Furthermore, mood states (especially anxious mood) had a stronger effect on risk and vulnerability than risk and vulnerability had on mood states. In the next chapter, the implications of this series of studies for RST will be discussed. 


\section{Chapter 7: General Discussion}

Five major findings from this series of studies challenge the applicability of RST to preadolescents and adolescents. First, although females consistently reported significantly more risk, vulnerability, and maladjustment during adolescence than males, the size of this gender difference was small enough to question the clinical significance of the result. Second, rumination was not encouraged disproportionately in females by their parents, nor did adolescents expect other females to ruminate more than males in response to distress. Third, rumination did not interact with stress to predict depressive symptoms during adolescence. Fourth, even though rumination acted as a concurrent mediator between stress and depressive symptoms, rumination was not as powerful a mediator as sense of control. And last, longitudinal results did not support this concurrent finding; longitudinally, vulnerability factors such as rumination, sense of control, and perceptions of false self did not moderate or mediate the relationship between stress and depressive symptoms across time. Instead, maladjustment-predominantly anxiety-predicted increases in risk and vulnerability across time. Interestingly, when the relationship between rumination and depression was measured in isolation, partial support was found for a bidirectional relationship among the variables. However, when additional variables were included in analyses, rumination and depression did not predict increases in each other across time.

Sex Differences in Risk, Vulnerability, and Maladjustment During Adolescence

RST was developed to help explain why females typically report higher levels of depression than males, thus from the outset, this theory adopts a gender differences perspective. A gender differences perspective, put simply, is one that assumes that meaningful differences exist between males and females and they require explanation. 
However, the trend of results reported in this thesis suggest that the differences between males and females on risk, vulnerability, and maladjustment may not be large enough to be clinically significant, even though they are statistically significant. Thus, the results of this thesis are more congruent with a gender similarities perspective. A gender similarities perspective has two major assertions, first, that males and females are similar on most but not all psychological constructs, and second, when sex differences occur they tend to be small (Hyde, 2005). The gender similarities hypothesis has a long history (Thorndike, 1914), yet it has often been overshadowed by the gender differences perspective in both the popular press and academic literature (Barnett \& Rivers, 2004). Literature examining maladjustment in adulthood and adolescence is no exception to this general rule, where conclusions are typically dominated by the gender differences perspective.

One factor that may have influenced the dominance of the gender difference perspective relates to the over reliance of null hypothesis testing within large samples, combined with a general lack of effect size reporting. The use of null hypothesis testing without effect sizes is an important limitation because the chances of finding a statistically significant result is increased substantially in large samples, even if the actual size of the difference is small (Kline, 2004). The following two sections will discuss how the over reliance on null hypothesis testing within large samples has exaggerated the differences that exist between males and females on constructs such as rumination and depression. Then, the implications of overemphasising sex differences will be discussed. I focus on the literature relating to sex differences in rumination and depression because RST was the primary focus of the current thesis. However, it is notable that the size of gender differences reported in this thesis on the constructs of 
stress, perceptions of false self, sense of control, and anxious symptoms are also small, and therefore the discussion is also relevant with respect to these constructs.

Sex differences in depression during adolescence. Many often cited studies have found that females begin to experience more depressive symptoms than males during adolescence (for example, Allgood-Merten et al., 1990; Hankin et al., 1998; Holsen, Kraft, \& Vittersø, 2000). In each case, conclusions have been based on large scale representative samples of adolescents, which are strengths of each study. However, very few studies have reported the effect sizes associated with these differences between males and females, thus it is unknown how large the differences are between males and females in these samples.

Two large scale studies (Jose \& Brown, 2007; Twenge \& Nolen-Hoeksema, 2002) that have reported effect sizes suggest the difference between males and females on depressive symptoms may be small. First, recall that Jose and Brown measured rumination and depressive symptoms in 1,218 adolescents aged 10-17 years old. They found that from 13 years old, females reported significantly more depressive symptoms than males. However, the effect size at each age group was very small; the highest effect (partial $\eta^{2}=.03$ ) was seen in 14 year olds (reported in Brown, 2003). Therefore, their results could be interpreted as supporting a gender similarities perspective because although the difference was statistically significant, the size of difference between males and females was very small.

Second, in their comprehensive meta-analysis, Twenge and Nolen-Hoeksema (2002) measured the average effect size of the sex difference in depressive symptoms across 310 samples. Recall that Twenge and Nolen-Hoeksema found that there was no sex difference prior to 13 years old, however 13 year old females reported more 
depressive symptoms than males, supporting a sex differences perspective. However, the average effect size for the gender difference in depressive symptoms (Cohen's $d=$ .16) was below the criteria for a small effect. The gender difference in depression reached the size of a small effect at ages 14 and 15 (Cohen's $d=.22$ for both ages). Twenge and Nolen-Hoeksema concluded that differences between males and female adolescents in the general population may not be clinically significant, thus these results could also be interpreted as supporting a gender similarities perspective. Sex differences in rumination. The studies that report gender differences in rumination can be criticized on the same grounds as those examining the gender differences in depression. That is, studies typically use large sample sizes, which increase the likelihood of significant results but do not report effect sizes. This criticism is true for research conducted with both adult samples (Butler \& NolenHoeksema, 1994; Conway et al., 2004; Garnefski et al., 2004; Nolan et al., 1998; Nolen-Hoeksema et al, 1999; Nolen-Hoeksema et al., 1994), and adolescent samples (Broderick, 1998; Broderick and Korteland, 2002; Driscoll, 2004; Muris, Roelofs et al., 2004; Schwartz and Koenig, 1996; Ziegert \& Kistner, 2002). Only one study (Jose \& Brown, 2007) has reported effect sizes in combination with significance tests. Recall, that Jose and Brown found that females started to report ruminating more than males from age 12, but the effects sizes were small; partial $\eta^{2}$ s ranged between .02 and .15 (reported in Brown, 2003), which are comparable to the effect sizes reported throughout this thesis. In combination, the results of this thesis along with those of Jose and Brown support a gender similarities perspective. In summary, when effect sizes are taken into account, it appears that females do report more rumination than males, but this difference tends to be small. 
The gender similarities perspective differs substantially to the prevailing view that female adolescents are at a heightened risk of maladjustment during adolescence (Hankin et al., 1998; Nolen-Hoeksema, 1994; Nolen-Hoeksema \& Girgus, 1994). However, a similar change in standpoint is emerging from researchers examining the sex differences in adolescent self-esteem. Traditionally, it has been argued that females experience a rapid deterioration in self-esteem compared to males during adolescence (Pipher, 1994; Orenstein, 1994). However in a recent meta-analysis, Kling, Hyde, Showers, and Buswell (1999) have disputed this claim by reviewing 216 samples involving 97,121 individuals. Kling et al. showed that there are sex differences in favour of males in self-esteem, but this gender difference tends to be small. Thus, Kling et al. concluded that the popular perspective that males and females differed substantially on self-esteem is unwarranted-recall Twenge and Nolen-Hoeksema (2002) reach a similar conclusion in relation to sex differences in depressive symptoms.

Unfortunately, a meta-analysis on gender differences in rumination has not been conducted for either adults or adolescents. Although beyond the scope of this thesis, it is important that future research focus on meta-analytic techniques to examine how large the average difference is between males and females on rumination. Based on the results of current data that reports effect sizes (current thesis; Jose \& Brown, 2007), it is predicted that the average size of effect between males and females on rumination would be small, thus supporting a gender similarities perspective. In this thesis, RST's tenet that females are more ruminative than males was statistically supported; however, the size of this difference may not be large in a 'real world' sense. 
The implications for adopting a gender difference perspective are potentially harmful for both male and female adolescents. For example, assuming males and females differ substantially on risk, vulnerability, and maladjustment may produce self-fulfilling prophecies for both males and females (Barnett \& Rivers, 2004; Kling et al., 1999). Potentially, if rumination and depressive symptoms are perceived as normative states for females, then inadequate preventive measures may be put in place to prevent females from ruminating and experiencing maladjustment in response to their problems. For example, parents, peers, or teachers may perceive rumination among females as girls being girls, which may prevent the discouragement of rumination among females.

For males, the potential consequence for the strongly asserted gender difference in risk, vulnerability, and maladjustment may be that males do not typically receive or request help for problems which may arise during adolescence (Barnett \& Rivers, 2004). For example, maladjustment may not be perceived as being as serious for males as for females, or males might be less inclined to discuss their feelings if emotional expression is perceived as stereotypically feminine. In adults, this perspective has being endorsed by Nolen-Hoeksema (2003), who in her book for the popular press, Women Who Think Too Much, targets females with the implication that rumination is less of a problem for males. In contrast, the gender similarities perspective asserts that risk, vulnerability, and maladjustment is experienced at a similar frequency for males and females during adolescence, thus encouraging an equal response to the behaviour and needs of both males and females. 
Response Styles Theory Applied to Adolescence: Does Rumination Prompt Depression?

RST as applied to adolescence is characterized as a diathesis-stress model

where the interaction of stress and vulnerability are expected to prompt the onset of depressive symptoms (Nolen-Hoeksema, 1994; Nolen-Hoeksema \& Girgus, 1994).

However, contrary to the predictions of RST, stress did not interact with rumination to predict depressive symptoms in adolescence either concurrently or across time, replicating the findings of Jose and Brown (2007) and Schwartz and Koenig (1996). Concurrently, rumination was found to mediate the relationship between stress and depressive symptoms, which adds to a growing body of research that suggests vulnerability factors may act as mediators rather than moderators of the relationship between stress and depressive symptoms (Cole \& Turner, 1993).

However, in subsequent longitudinal tests, rumination did not mediate the relationship between stress and depressive symptoms, suggesting that the relationships among risk, vulnerability, and maladjustment have an alternative causal pathway (direction of effect). To date, most studies assessing the relationships among risk, vulnerability, and maladjustment have assumed that risk and vulnerability increase maladjustment. This assumption is evident in the fact that the majority of studies reviewed in this thesis have predominantly tested only one direction of effect, namely from risk and vulnerability to maladjustment. For example, the path from rumination to depression has been the primary focus in both adult (Butler \& Nolen-Hoeksema, 1994; Just \& Alloy, 1997; Nolen-Hoeksema, 2000; Nolen-Hoeksema \& Morrow, 1991; Nolen-Hoeksema et al., 1993) and adolescent samples (Abela et al., 2002; Driscoll, 2004; Swartz \& Koenig, 1996). Interestingly, when studies have tested alternative directions of effect, the results have found support for bidirectional 
relationships (Nolen-Hoeksema, Stice, et al., 2007; Weir et al., 2006); yet even when bidirectional relationships are found, the direction of effect tends to be stronger from maladjustment to vulnerability than the reverse. For example, even when a bidirectional relationship has been found between rumination and depressive symptoms, depressive symptoms have a stronger effect on rumination than rumination has on depressive symptoms.

The current thesis supports the position that the direction of effect is predominantly from maladjustment to risk and vulnerability. Across 10 weeks, maladjustment predicted increases in stress, sense of control, and perceptions of false self, whereas none of the risk or vulnerability factors predicted changes in later maladjustment. Extending upon these results, anxious and depressive symptoms were then considered separately in study 3 to assess potentially distinct relationships with risk and vulnerability across 4 months. This approach was fruitful in that distinguishing between anxious and depressive symptoms highlighted an important finding-anxious symptoms appear to drive dysfunctional changes in stress, perceptions of false self, rumination, sense of control, and depressive symptoms. In short, anxiety predicted unique changes in all other measured variables across 4 months. By comparison, depressive symptoms only predicted decreases in sense of control across time when anxious symptoms were considered in the analyses. Therefore it appears that it is a specific type of maladjustment-specifically anxiety-that is prompting increases in risk and vulnerability across time.

Across 10 weeks, when rumination and depression were measured in isolation, partial support for a bidirectional relationship between rumination and maladjustment was found, but the relationship was not in the direction that RST 
would predict-maladjustment marginally predicted increases in rumination, but rumination did not predict increases in maladjustment. When additional risk and vulnerability were added to this model, the relationship between maladjustment and rumination was eliminated.

This result was replicated in Chapter 6 where partial support was also found for a bidirectional relationship between rumination and depression; however, again the effect of depression on rumination was stronger than the effect of rumination on depression. When these variables were no longer assessed in isolation, this relationship disappeared, further replicating the results of study 2. Interestingly, in both previous studies which have found a bidirectional relationship between rumination and depressive symptoms (Nolen-Hoeksema, Stice et al. 2007; Weir et al., 2006) rumination and depressive symptoms were examined in the absence of additional variables. In these former studies no additional variables were competing for unique variance in maladjustment. The results of this thesis therefore suggest that the relationship between rumination and depressive symptoms found previously may be accounted for by the relationships of rumination and depressive symptoms with other variables (so-called third variables).

In support of this argument, Arnow et al. (2004) examined the effects of rumination, distraction, and depression across 12 weeks on patients recovering from a major depressive episode. Arnow et al. tested whether rumination led to depression as RST stipulates, whether depression led to rumination, or if the relationship was accounted for by a common cause model. Arnow et al.'s results indicated that rumination did not lead to later depression, and likewise depression did not lead to later rumination. Rather, these data were best described through a common cause or 
spurious effect model. Thus, these data were consistent with the hypothesis that the relationship between rumination and depression was the result of a third, unknown variable. Unfortunately in Arnow et al.'s study, additional variables to rumination and depression were not actually measured, so this study was unable to suggest what sorts of third variables may be involved.

The results of the current thesis suggest that a potential third variable could be anxiety. In this thesis anxiety was found to predict increases in both depressive symptoms and rumination across time. Consequently, the bi-directional relationship between rumination and depressive symptoms seen when measured in isolation could occur because both variables are associated with anxiety, which in turn appears to prompt increases in both rumination and depression. The association between depressive and anxious symptoms is well established-in fact Ingram (1990) has argued that depressive symptoms are rarely experienced without concurrent anxious symptoms. Similarly, research shows that when an individual experiences rumination, they are also likely to experience anxious symptoms (Driscoll, 2004; NolenHoeksema, 2000). In addition, rumination is conceptually similar to the predominant feature of anxiety-worry-in that an individual who ruminates experiences a sense of uncertainty about the future, which is expressed in items on the RSQ, for example, "I won't be able to concentrate if I keep feeling this way".

Research that has examined how the relationship between rumination and depressive symptoms is affected by anxious symptoms also supports a spurious hypothesis. For example, in undergraduate samples, the relationship between rumination and depressive symptoms has been found to substantially reduce when levels of anxiety are controlled (Harrington \& Blankenship, 2002; Segerstrom, Tsao, 
Alden, \& Craske, 2000). Furthermore, Weir and Jose (2004) found that the relationship between rumination and depressive symptoms was fully mediated by anxious symptoms in a sample of undergraduate students. In other words, the relationship between rumination and depressive symptoms was reduced to nonsignificance when anxious symptoms were controlled. Similar results have recently been found with adolescents. For example, Muris, Roelofs, et al. (2004) found that the relationship between rumination and depressive symptoms was reduced to nonsignificance when anxiety was controlled.

However, a spurious association with anxiety cannot be the only reason why the bidirectional relationship between rumination and depressive symptoms was not found. Anxiety cannot be the only reason mainly because the relationship between rumination and depression did not reoccur, when anxiety was omitted from the model. This latter finding raises three alternative explanations. First, the relationship between rumination and depression may exist legitimately, however the strength of the relationship is so weak that when additional variables are included in analyses that require very large sample sizes, such as structural equation modelling, the effects become non-significant. However, this is not likely to solely explain the result because the relationship between rumination and depression has been removed with the inclusion of anxiety using analyses less reliant on large sample size (see Muris, Roelofs et al., 2004).

Second, anxiety may not be the only third variable which affects the relationship between rumination and anxiety. Another possible contributor to both rumination and depressive symptoms is stress. This possibility is evident in the finding seen in Figure 6.6 when anxiety is not included in the model and stress predicts 
changes in both rumination and depressive symptoms. In this sense, stress increases both rumination and depression, and thus the bi-directional relationship evident in previous research may be spuriously prompted by stress. However, given that stress is also predicted by anxiety, anxiety is still likely to be the strongest factor influencing the relationship between rumination and depression. Another possibility is that multiple third variables may also be involved, in addition to the variables measured in this thesis.

Third, it may be that the relationship between rumination and depression during adolescence takes longer to become evident than four months, for example, the effect of rumination on depressive symptoms was evident in adolescents across 4 years in Nolen-Hoeksema, Stice et al.'s (2007) study. However, there is no theoretical reason as to why rumination's effect on depressive symptoms would take this long to occur, and previous studies have found that rumination predicts change in depressive symptoms across shorter periods of time, for example, across 6 weeks (Abela et al., 2002).

The most likely explanation as to why the bidirectional relationship between rumination and depression disappears with the inclusion of additional variables is a combination of these alternative explanations. Multiple spurious associations are likely to be at play-two likely third variables are stress and anxiety. And these spurious factors appear to impact on the already weak longitudinal relationship between rumination and depression. In other words, the relationship between rumination and depression in a focused analysis appears to be weak, and this appears to disappear when other variables are included. In summary, little support was found for RST's prediction that rumination exacerbates depression. Incongruent with the predictions 
of RST, in the present datasets, the relationship between rumination and depression appeared to be weak and was completely eliminated by related constructs such as stress and anxiety Applications to Clinical Depression

In this thesis depressive symptoms were measured using the Children's Depression Inventory. Although this measure is a reliable and valid measure of depressive symptoms, it alone cannot diagnose depression, and thus it cannot be assumed that the adolescents who scored highly on this measure were in fact depressed. This thesis did not assess the applicability of RST to clinical depression during adolescence, instead, RST was tested in a sample of non-clinical adolescents from the general population. Predominantly, this sample was collected to be consistent with existing research examining RST, which relies heavily on non-clinical populations (for example, Abela et al., 2002; Nolen-Hoeksema et al., 1999; Ziegert \& Kistner, 2002).

However, theoretically RST purports that rumination has a similar effect on maladjustment regardless of the severity of depressed mood. That is, rumination should exacerbate and prolong clinical levels of depression, as well as sub-clinical levels of depression as studied here. The results for RST's application in clinical populations are mixed. For example with adults, Arnow et al.'s (2004) findings did not support RST's application in a clinical population, whereas Kuehner and Weber's (1999) results did. It is unknown whether RST would be supported in clinical samples of adolescents, therefore future studies could test the applicability of RST in clinically depressed adolescents.

Age as a Proxy to Stage of Development 
Throughout this thesis, age has been used to approximate the stage of development. For example 9-12 years old were considered preadolescents, and 13-17 year olds were considered adolescents. This is however, an imperfect indicator of the physical and cognitive changes that occur with the onset of adolescence. In the future studies should gather indicators of pubertal development, such as onset of menstruation, and/or cognitive indicators to determine developmental stage. Measuring these more subtle indicators of development should produce a more appropriate test of the developmental predictions of RST.

Representativeness of Sample

Across all three studies there was a lack of representativeness of participants from low socio-economic (low decile) schools. Schools that were willing to participate in this research tended to be from middle to high socio-economic backgrounds, and furthermore, when low decile schools were recruited return rates of consent forms were particularly low. As a result, the results of this thesis may not reflect the associations among stress, vulnerability, and maladjustment among adolescents from low socio-economic backgrounds. Future research could assess if socio-economic status impacts upon the relationships among stress, vulnerability, and maladjustment found in this thesis.

Conclusion

The landscape of general psychological distress during adolescence includes a combination of dysfunction such as higher stress, rumination, perceptions of false self, anxious and depressive symptoms, and less perceived control over one's life. Within this landscape, a specific type of maladjustment-anxiety-appears to sustain one's overall feelings of distress. RST predicts that the combination of stress and rumination 
may prompt and maintain depressive symptoms in adolescence; however the results of this thesis did not support such a claim. Furthermore, the two major tenets of RST were not supported across a series of three studies with adolescents. First although female adolescents exhibited a slight tendency to ruminate more than male adolescents, this tendency was small, supporting a gender similarities perspective. And second, although rumination was associated with current levels of distress, rumination did not predict changes in depressive symptoms across time, contrary to the predictions of RST. The relationship found in previous research between rumination and depression appears to be, in part, a function of both variables' association with stress and anxiety. Thus, the stress and worry that is potentially captured while ruminating may be the driving force behind depression-rather than rumination itself. 


\section{References}

Abela, J. R. Z., Broniza, K., \& Haigh, E. P. (2002). An examination of response styles theory of depression in third- and seventh-grade children: A short-term longitudinal study. Journal of Abnormal Child Psychology, 30, 515-527.

Allgood-Merten, B., Lewinsohn, P. M., \& Hops, H. (1990). Sex differences and adolescent depression. Journal of Abnormal Psychology, 99, 55-63.

Anderson, J. C., \& Gerbing, D. W. (1988). Structural equation modeling in practice: A review and recommended two-step approach. Psychological Bulletin, 103, 411-423.

Arnow, B. A., Spangler, D., Klein, D. N., \& Burns, D. D. (2004). Rumination and distraction among chronic depressives in treatment: A structural equation analysis. Cognitive Therapy and Research, 28, 67-83.

Bandalos, D. L., \& Finney, S. J. (2001). Item parceling issues in structural equation modeling. In G. A. Marcoulides \& R. E. Schumacker (Eds.), Advanced structural equation modeling: New developments and techniques. Mahwah, NJ; Lawrence Erlbaum Associates, Inc.

Bandura, A. (1986). Social foundations of thought and action: A social cognitive theory. Englewood Cliffs, NJ: Prentice-Hall.

Barnett, R., \& Rivers, C. (2004). Same difference: How gender myths are hurting our relationships, our children, and our jobs. New York: Basic Books.

Baron, R. M., \& Kenny, D. A. (1986). The moderator-mediator variable distinction in social psychological research: Conceptual, strategic, and statistical considerations. Journal of Personality and Social Psychology, 51, 1173-1182. 
Baumeister, R. F. (1987). How the self became a problem: A psychological review of historical research. Journal of Personality and Social Psychology, 52, 163-176.

Beck, A. T., Brown, G., Epstein, N., \& Steer, R. A. (1988) An inventory for measuring clinical anxiety: psychometric properties. Journal of Consulting and Clinical Psychology, 56, 893-897.

Beck, A. T., \& Steer, R. A. (1993) Beck Anxiety Manual. San Antonio: the Psychological Corp.

Bem, S. L. (1974). The measurement of psychological androgyny. Journal of Consulting and Clinical Psychology, 42, 155-162.

Besser, A., Flett, G. L., \& Davis, R. A. (2003). Self-criticism, dependency, silencing the self, and loneliness: A test of a mediational model. Personality and Individual Differences, 35, 1735-1752.

Blagden, J. C., \& Craske, M. G. (1996). Effects of active and passive rumination and distraction: A pilot replication with anxious mood. Journal of Anxiety Disorders, $10,243-252$.

Broderick, P. C. (1998). Early adolescent gender differences in the use of ruminative and distracting coping strategies. Journal of Early Adolescence, 18, 173-191.

Broderick, P.C., Korteland, C. (2002). Coping style and depression in early adolescence: Relationships to gender, gender-role, and implicit beliefs. Sex Roles, 46, 201-213.

Broderick, P.C., \& Korteland, C. (2004). A prospective study of rumination and depression in early adolescence. Clinical Child Psychology and Psychiatry, 9, 383394. 
Brown, I. K. (2003). The development of rumination in New Zealand adolescents: Is rumination a maladaptive coping stategy? Unpublished master's thesis, Victoria University of Wellington, Wellington, New Zealand.

Bryant, F. B., \& Yarnold, P. R. (1995) Principal-components analysis and exploratory and confirmatory factor analysis. In L. G. Grimm, \& P. R. Yarnold (Eds.). Reading and understanding multivariate statistics (pp. 99-137). Washington: American Psychological Association.

Butler, L. D., \& Nolen-Hoeksema, S. (1994). Gender differences in response to depressed mood in a college sample. Sex Roles, 30, 331-346.

Carlson, G. A., \& Kashani, J. H. (1988). Phenomenology of major depression from childhood through adulthood: Analysis of three studies. American Journal of Psychiatry, 145, 1222-1225.

Cohen, J. (1992). A Power Primer. Psychological Bulletin, 112, 155-159.

Cole, D. A. (1987). Utility of confirmatory factor analysis in test validation research. Journal of Consulting and Clinical Psychology, 55, 584-594.

Cole, D. A., \& Turner, J. E. (1993). Models of cognitive mediation and moderation in child depression. Journal of Abnormal Psychology, 102, 271-281.

Compas, B. E., \& Phares, V. (1991). Stress during childhood and adolescence: Sources of risk and vulnerability. In M. E. Cummings, A. L. Greene, \& K. H. Karraker (Eds), Life-span Developmental Psychology: Perspectives on Stress and Coping (pp. 111-129). Hillsdale, NJ: Lawrence Erlbaum Associates. 
Conway, M., Csank, P. A. R., Holm, S. L., \& Blake, C. K. (2000). On assessing individual differences in rumination on sadness. Journal of Personality Assessment, 75, 404-425.

Conway, M., Mendelson, M., Giannopoulos, C., Csank, P. A. R., \& Holm, S. L. (2004). Childhood and adult sexual abuse, rumination on sadness, and dysphoria. Child Abuse and Neglect, 28, 393-410.

Cramer, K. M., Gallant, M. D., \& Langlois, M. W. (2005). Self-silencing and depression in women and men: Comparative structural equation models. Personality and Individual Differences, 39, 581-592.

Cramer, K. M., \& Thoms, N. (2003). Factor structure of the silencing the self scale in women and men. Personality and Individual Differences, 35, 525-535.

DeLongis, A. (1982). Relationship of daily hassles, uplifts, and major life events to health status. Health Psychology, 1, 119-136.

Donnelly, M. (1999). Factors associated with depressed mood among adolescents in Northern Ireland. Journal of Community and Applied Social Psychology, 9, 47-59.

Driscoll, K. A. (2004). Children's response styles and risk for depression and anxiety: developmental and sex differences. Unpublished doctoral dissertation, Florida State University College of Arts and Sciences, Florida.

Duarte, L. M., \& Thompson, J. M. (1999). Sex differences in self-silencing. Psychological reports, 85, 145-161.

Fischer, K. W. (1980). A theory of cognitive development: The control and construction of hierarchies of skills. Psychological Review, 87, 477-531. 
Flett, G. L., Madorsky, D., Hewitt, P. L., \& Heisel, M. J. (2002). Perfectionism cognitions, rumination, and psychological distress. Journal of Rational-Emotive and Cognitive-Behavior Therpay, 2, 33-47.

Flick, U. (2006). An introduction to qualitative research ( ${ }^{\text {rd }}$ ed.). London: Sage Publications.

Garber, J., \& Flynn, C. (2001). Vulnerability to depression in childhood and adolescence. In R. E. Ingram \& J. M. Price (Eds.), Vulnerability to Psychopathology (pp. 175-225). New York: Guilford Press.

Garnefski, N., Boon, S., \& Kraaij, V. (2003). Relationships between cognitive strategies of adolescents and depressive symptomatology across different types of life event. Journal of Youth and Adolescence, 32, 401-408.

Garnefski, N., Kraaij, V., \& Spinhoven, P. (2001). Negative life events, cognitive emotion regulation and emotional problems. Personality and Individual Differences, 30, 1311-1327.

Garnefski, N., Teerds, J., Kraaij, V., Legerstee, J., \& Van den Kommer, T. (2004). Cognitive emotion regulation strategies and depressive symptoms:

Differences between males and females. Personality and Individual Differences, $36,267-276$.

Gibb, B. E., \& Alloy, L. B. (2006). A prospective test of the hopelessness theory of depression in children. Journal of Clinical Child and Adolescent Psychology, 35, 264-274.

Giles, D. C. (2002). Advanced research methods in psychology. New York: Routledge. 
Gilligan, C. (1993). Joining the resistance: Psychology, politics, girls, and women. In L. Weis \& M. Fine (Eds.), Beyond silenced voices (pp. 143-168). Albany, NY: State University of New York Press.

Gotlib, I. H. (1984). Depression and general psychopathology in university students. Journal of Abnormal Psychology, 93, 19-30.

Grant, K. E., Compas, B. E., Thurm, A. E., McMahon, S. D., Gipson, P. Y., Campbell, A. J., Krochock, K., \& Westerholm, R. I. (2006). Stressors and child and adolescent psychopathology: Evidence of moderating and mediating effects. Clinical Psychology Review, 26, 257-283.

Hammen, C. L. (2005, May). Interpersonal stress and depression. Paper presented at the American Psychological Society $17^{\text {th }}$ Annual Convention, Los Angeles, CA.

Hankin, B. L., \& Abramson, L. Y (1999). Development of gender differences in depression: description and possible explanations. Annual Medicine, 31, 372 379.

Hankin, B. L., Abramson, L.Y., Moffitt, T. E., Silva, P. A., McGee, R., \& Angell, K. E. (1998). Development of depression from preadolescence to young adulthood: Emerging gender differences in a 10-year longitudinal study. Journal of Abnormal Psychology, 107, 128-140.

Harrington, J. A., \& Blankenship, V. (2002). Ruminative thoughts and their relation to depression and anxiety. Journal of Applied Social Psychology, 32, 465-485.

Harrington, R., Fudge, H., Rutter, M., Pickles, A., \& Hill, J. (1990). Adult outcomes of childhood and adolescent depression, I: Psychiatric status. Archives of General Psychiatry, 41, 465-473. 
Harter, S. (1985). Manual for the self-perception profile for children. Denver, CO:

University of Denver.

Harter, S. (1999). The construction of the self: A developmental perspective. New York: Guilford Press

Harter, S. (2002). Authenticity. In C. R. Snyder \& J. Lopez (Eds.). Handbook of positive psychology. (382-394). Oxford, University Press.

Harter, S., Bresnick, S., Bouchey, H. A., \& Whitesell, N. R. (1997). The development of multiple role-related selves during adolescence. Development and Psychopathology, 9, 835-853.

Harter, S., Marold, D. B., Whitesell, N. R., \& Cobbs, G. (1996). A model of the effects of perceived parent and peer support on adolescent false self behavior. Child Development, 67, 360-374.

Harter, S., \& Monsour, A. (1992). Developmental analysis of conflict caused by opposing attributes in the adolescent self-portrait. Developmental Psychology, $28,251-260$.

Harter, S. \& Waters, P. L. (1991). Saying What I Think Around Others. Unpublished manuscript. Denver, CO; University of Denver.

Harter, S., Waters, P. L., \& Whitesell, N. R. (1997). Lack of voice as a manifestation of false self-behavior among adolescents: The school setting as a stage upon which the drama of authenticity is enacted. Educational Psychologist, 32, 153173.

Higgins, E. T. (1987). Self-discrepancy: A theory relating self and affect. Psychological Bulletin, 94, 319-340. 
Hill, J.P., \& Lynch, M. E. (1983). The intensification of gender-related role expectations during early adolescence. In J. Brooks-Gunn \& A. C. Peterson (Eds.), Girls at Puberty (pp. 201-228). New York: Plenum Press.

Holmbeck, G. N. (1997). Toward terminological, conceptual, and statistical clarity in the study of mediators and moderators: Examples from the child-clinical and pediatric psychology literature. Journal of Consulting and Clinical Psychology, 65, 599-610

Holsen, I., Kraft, P., \& Vittersø, J. (2000). Stability in depressed mood in adolescence: Results from a 6-year longitudinal panel study. Journal of Youth and Adolescence, 29, 61-78.

Howell, D. C. (2002). Statistical methods for psychology ( $5^{\text {th }}$ ed.). Pacific Grove, CA: Duxbury.

Hu, L., \& Bentler, P. M. (1999). Cuttoff criteria for fit indices in covariance structure analysis: Conventional criteria versus new alternatives. Structural Equation Modeling, 6, 1-55.

Huston, A. C., \& Alvarez, M. M. (1990). The socialization context of gender role development in early adolescence. In R. Montgomery, \& G. R. Adams (Eds.). From childhood to adolescence (pp. 156-179). Newbury Park, CA: Sage.

Hyde, J. S. (2005). The gender similarities hypothesis. American Psychologist, 60, 581 592.

Ingram, R. E. (1990). Self-focused attention in clinical disorders: Review and conceptual model. Psychological Bulletin, 107. 156-176. 
Ingram, R. E., \& Price, J. M. (2001). The role of vulnerability in understanding psychopathology. In R. E. Ingram \& J. M. Price (Eds.), Vulnerability to Psychopathology (pp. 3-19). New York: Guilford Press.

Jack, D. C. (1991). Silencing the Self: Women and Depression. Cambridge: Harvard University Press.

Jack, D. C., \& Dill, D. (1992). The silencing the self scale: Schemas of intimacy associated with depression in women. Psychology of Women Quarterly, 16, 97106.

James, W. (1890). The Principles of Psychology (2 vols.). New York: Henry Holt (Reprinted Bristol: Thoemmes Press, 1999).

Jolly, J. B., Aruffo, J. F., Wherry, J. N., \& Livingston, R. (1993). The utility of the Beck Anxiety Inventory with inpatient adolescents. Journal of Anxiety Disorders, 7, 95-106.

Jose, P.E. (2003). Medgraph: A programme to graphically depict mediation among three variables: The internet version. Wellington, New Zealand: Victoria University of Wellington. http://www.vuw.ac.nz/psyc/staff/pauljose/files/medgraph/medgraph.php. Retrieved 12.12.05.

Jose, P. E., \& Brown, I. (in press). When does the gender difference in rumination begin? Gender and age differences in the use of rumination by adolescents. Journal of Youth and Adolescence.

Jose, P. E., Cafasso, L. L., \& D’Anna, C. A. (1994). Ethnic group differences in children's coping strategies. Sociological Studies of Children, 6, 25-53.

Just, N., \& Alloy, L. B. (1997). The response styles theory of depression: Tests and an extension of the theory. Journal of Abnormal Psychology, 106, 221-229. 
Kandel, D. B., \& Davies, M. (1986). Adult sequelae of adolescent depressive symptoms. Archives of General Psychiatry, 43, 255-262.

Katz, E. J., \& Bertelson, A. D. (1993). The effects of gender and response style on depressed mood. Sex Roles: A Journal of Research, 29, 509-514.

Kessler, R. C., McGonagle, K. A. Swartz, M., Blazer, D. G., \& Nelson, C. B. (1993). Sex and depression in the national comorbidity survey I: Liketime prevalence, chronicity and recurrence. Journal of Affective Disorders, 29, 85-96

Kline, R. B. (1998). Principles and practices of structural equation modeling. New York: The Guilford Press.

Kline, R. B. (2004). Beyond significance testing: Reforming data analysis methods in behavioral research. Washington DC: American Psychological Association

Kline, R. B. (2005). Principles and practices of structural equation modeling $\left(2^{\text {nd }}\right.$ ed.). New York: The Guilford Press.

Kling, K. C., Hyde, J. S., Showers, C. J., \& Buswell, B. N. (1999). Gender differences in self-esteem: A meta-analysis. Psychological Bulletin, 125, 470500.

Kovacs, M. (1985). The Children's Depression Inventory (CDI). Psychopharmacology, 21, 995-998.

Kovacs, M. (1992). Children’s Depression Inventory Manual. New York: Multi-Health Systems Inc.

Kraaij, V., Garnefski, N., Jan de Wilde, E., Dijkstra, A., Gebhardt, W., Maes, S., \& ter Doest, L. (2003). Negative life events and depressive symptoms in late 
adolescence: Bonding and cognitive coping as vulnerability factors? Journal of Youth and Adolescence, 32, 185-193.

Kuehner, C. (2003). Gender differences in unipolar depression: An update of epidemiological findings and possible explanations. Acta Psychitrica Scandinavica, 108, 163-174.

Kuehner, C., \& Weber, I. (1999). Responses to depression in unipolar depressed patients: An investigation of Nolen-Hoeksema's Response Styles Theory. Psychological Medicine, 29, 1323-1333.

Leff, S. S., Crick, N. R., Angelucci, J., Haye, K., Jawad, A. F., Grossman, M., \& Power, T. J. (2006). Social cognition in context: Validating a cartoon-based attributional measure for urban girls. Child Development, 77, 1351-1358.

Lobel, T. E., Nov-Krispin, N., Schiller, D., Lobel, O., \& Feldman, A. (2004).

Gender discriminatory behavior during adolescence and young adulthood: A developmental analysis. Journal of Youth and Adolescence, 33, 535-546.

Little, T. D., Cunningham, W. A., Shahar, G., \& Widaman, K. F. (2002). To parcel or not to parcel: Exploring the question, weighing the merits. Structural Equation Modeling, 9, 151-173.

Lyubomirsky, S., \& Nolen-Hoeksema, S. (1993). Self-perpetuating properties of dysphoric rumination. Journal of Personality and Social Psychology, 65, 339-349. Lyubomirsky, S., \& Nolen-Hoeksema, S. (1995). Effects of self-focused rumination on negative thinking and interpersonal problem solving. Journal of Personality and Social Psychology, 69, 176-190. 
Lyubomirsky, S. \& Tkach, C. (2004). The consequences of dysphoric rumination. In C. Papageorgiou \& A. Wells (Eds.), Depressive Rumination: Nature, Theory and Treatment (pp. 21-41). West Sussex: John Wiley \& Sons Ltd.

Marsh, H. W., \& Hocevar, D. (1985). Application of confirmatory factor analysis to the study of self-concept: First- and higher order factor models and their invariance across groups. Psychological Bulletin, 97, 562-582.

Martin, C. L., \& Ruble, D. (2004). Children's search for gender cues: Cognitive perspectives on gender development. Current Directions in Psychological Science, 13, 67-70.

Mor, N., \& Winquist, J. (2002). Self-focused attention and negative affect: A metaanalysis. Psychological Bulletin, 128, 638-662.

Morrison, R., \& O’Connor, R. C. (2005). Predicting psychological distress in college students: The role of rumination and stress. Journal of Clinical Psychology, 61, 447-460.

Morrow, J., \& Nolen-Hoeksema, S. (1990). Effects of responses to depression on the remediation of depressive affect. Journal of Personality and Social Psychology, 58, 519-527.

Muris, P., Roelofs, J., Meesters, C., \& Boomsma, P. (2004). Rumination and worry in nonclinical adolescents. Cognitive Therapy and Research, 28, 539-554.

Muris, P., Meesters, C., Schouten, E., \& Hoge, E. (2004). Effects of perceived control on the relationship between perceived parental rearing behaviors and symptoms of anxiety and depression in nonclinical preadolescents. Journal of Youth and Adolescence, 33, 51-58. 
Neff, K. D., \& Harter, S. (2002). The role of power and authenticity in relationship styles emphasizing autonomy, connectedness, or mutuality among adult couples. Journal of Social and Personal Relationships, 19, 835-857.

Nolan, S. A., Roberts, J. E., \& Gotlib, I. H. (1998). Neuroticism and ruminative response styles as predictors of change in depressive symptomatology. Cognitive Therapy and Research, 22, 445-455.

Nolen-Hoeksema, S. (1987). Sex differences in unipolar depression: Evidence and theory. Psychological Bulletin, 101, 259-282.

Nolen-Hoeksema, S. (1990). Sex Differences in Depression. Stanford, California: Stanford University Press.

Nolen-Hoeksema, S. (1991). Responses to depression and their effects on the duration of depressive episodes. Journal of Abnormal Psychology, 100, 569-582.

Nolen-Hoeksema, S. (1994). An interactive model for the emergence of gender differences in depression in adolescence. Journal of Research on Adolescence, 4, 519-534.

Nolen-Hoeksema, S. (2000). The role of rumination in depressive disorders and mixed anxiety/depressive symptoms. Journal of Abnormal Psychology, 109, 504511.

Nolen-Hoeksema, S. (2003). Women who think too much: How to break free of overthinking and reclaim your life. New York: Henry Holt and Company.

Nolen-Hoeksema, S. \& Girgus, J. S. (1994). The emergence of gender differences in depression during adolescence. Psychological Bulletin, 115, 424-443.

Nolen-Hoeksema, S., \& Jackson, B. (2001). Mediators of the gender difference in rumination. Psychology of Women Quarterly, 25, 37-47. 
Nolen-Hoeksema, S., Larson, J., \& Grayson, C. (1999). Explaining the gender difference in depressive symptoms. Journal of Personality and Social Psychology, 77, 1061-1072.

Nolen-Hoeksema, S., McBride, A., \& Larson, J. (1997). Rumination and psychological distress among bereaved partners. Journal of Personality and Social Psychology, 72, 855-862.

Nolen-Hoeksema, S., \& Morrow, J. (1991). A prospective study of depression and posttraumatic stress symptoms after a natural disaster: The 1989 Loma Prieta earthquake. Journal of Personality and Social Psychology, 61, 115-121.

Nolen-Hoeksema, S., \& Morrow, J. (1993). Effects of rumination and distraction on naturally occurring depressed mood. Cognition and Emotion, 7, 561-570.

Nolen-Hoeksema, S., Morrow, J., \& Fredrickson, B. L. (1993). Response styles and the duration of episodes of depressed mood. Journal of Abnormal Psychology, 102, 20-28.

Nolen-Hoekema, S., Parker, L. E., \& Larson, J. (1994). Ruminative coping with depressed mood following loss. Journal of Personality and Social Psychology, 67, 92-104.

Nolen-Hoeksema, S., Stice, E., Wade, E., \& Behon, C. (2007). Reciprocal relations between rumination and bulimic, substance abuse, and depressive symptoms in female adolescents. Journal of Abnormal Psychology, 116, 198-207.

Nolen-Hoeksema, S., Wisco, B. E., \& Lyubomirsky, S. (in press). Rethinking Rumination. Perspectives on Psychological Science. 
Page, J. R., Stevens, H. B., \& Galvin, S. L. (1996). Relationships between depression, self-esteem, and self-silencing behaviour. Journal of Social and Clinical Psychology, 15, 381-396.

Pett, M. A., Lackey, N. R., \& Sullivan, J. J. (2003). Making sense of factor analysis: The use of factor analysis for instrument development in health care research. California: Sage Publications.

Pipher, M. (1994). Reviving Ophelia: Saving the Selves of Adolescent Girls. USA: Ballantine Books

Price, J. M., \& Lento, J. (2001). The nature of child and adolescent vulnerability: History and definitions. In R. E. Ingram \& J. M. Price (Eds.), Vulnerability to Psychopathology (pp. 20-38). New York: Guilford Press.

Pyszczynski, T., \& Greenberg, J. (1987). Self-regulatory preservation and the depressive self-focusing style: A self-awareness theory of reactive depression. Psychological Bulletin, 102, 122-138.

Reynolds, C. R., \& Richmond, B. O. (1997). What I think and feel: A revised measure of Children's Manifest Anxiety. Journal of Abnormal Child Psychology, 25, $15-20$.

Roberts, J. E., Gilboa, E., \& Gotlib, I. H. (1998). Ruminative response style and vulnerability to episodes of dysphoria: Gender, neuroticism, and episode duration. Cognitive Therapy and Research, 22, 401-423.

Robinson, S. M., \& Alloy, L. B. (2003). Negative inferential style and stress-reactive rumination. Interative risk factors in the etiology of depression. Cognitive Therapy and Research, 27, 275-291. 
Rose, A. J. (2002). Co-rumination in the friendships of girls and boys. Child Development, 73, 1830-1843.

Ruble, D. N., Eisenberg, R., \& Higgins, E. T. (1994). Developmental changes in achievement evaluation: Motivational implication of self-other differences. Child Development, 65, 1095-1110.

Sarin, S., Abela, J. R. Z., \& Auerbach, R. P. (2005). The response styles theory of depression: A test of specificity and causal mediation. Cognition \&Emotion, 19, $751-761$.

Saylor, C. F., Finch, A .J., Jr., Spirito, A., \& Bennet, B. (1984). The Children’s Depression Inventory: A systematic evaluation of psychometric properties. Journal of Consulting and Clinical Psychology, 52, 955-967.

Scher, C. D., Ingram, R. E., \& Segal, Z. V. (2005). Cognitive reactivity and vulnerability: Empirical evaluation of construct activation and cognitive diatheses in unipolar depression. Clinical Psychology Review, 25, 487-510.

Schumacker, R. E., \& Lomax, R. G. (1996). A beginner's guide to structural equation modeling. New Jersey: Lawrence Erlbaum Associates, Publishers.

Schwartz, J. A. J., \& Koenig, L. J. (1996). Response styles and negative affect among adolescents. Cognitive Therapy and Research, 20, 13-36.

Segerstrom, S. C., Tsao, J. C. I., Alden, L. E., \& Craske, M. G. (2000). Worry and rumination: Repetitive thought as a concomitant and predictor of negative mood. Cognitive Therapy and Research, 24, 671-688.

Shapiro, D. H., Jr. (1994). Shapiro Control Inventory. San Jose: Behaviordata, Inc.

Smolak, L., \& Munstertieger, F. (2002). The relationship of gender and voice to depression and eating disorders. Psychology of Women Quarterly, 26, 234-241. 
Spasojevic, J., Alloy, L. B., Abramson, L. Y., MacCoon, D., \& Robinson, M. S. (2004). Reactive rumination: Outcomes, mechanisms, and developmental antecedents. In C. Papageorgiou \& A. Wells (Eds.), Depressive Rumination: Nature, Theory and Treatment (pp. 43-58). West Sussex: John Wiley \& Sons Ltd.

Strauss, J., Muday, T., McNall, K., \& Wong, M. (1997). Response styles theory revisited: Gender differences and stereotypes in rumination and distraction. Sex Roles, 36, 771-792.

Stevens, E. A., \& Prinstein, M. J. (2005). Peer contagion of depressogenic attributional styles among adolescents: A longitudinal study. Journal of Abnormal Child Psychology, 33, 25-37.

Tabachnick, B. G., \& Fidell, L. S. (2001). Using multivariate statistics $\left(4^{\text {th }}\right.$ Ed.). Boston, MA: Allyn \& Bacon.

Tanner, J. M. (1990). Foetus into man: Physical growth from conception to maturity $\left(2^{\text {nd }}\right.$ ed.). Cambridge, MA: Harvard University Press.

Teasdale, J. D. (1983). Negative thinking in depression: cause, effect, or reciprocal relationships? Advances in Behaviour Research and Therapy, 5, 3-25.

Thompson, B. (2004). Exploratory and confirmatory factor analysis: Understanding concepts and application. Washington DC: American Psychological Association.

Thorndike, E. L. (1914). Educational Psychology (Vol. 3). New York: Columbia University. 
Trautner, H. M., Ruble, D. N., Cyphers, L., Kirsten, B., Behrendt, R., \& Hartmann, P. (2005). Rigidity and flexibility of gender stereotypes in childhood:

Developmental or differential? Infant and Child Development, 14, 265-381.

Treynor, W., Gonzalez, R., \& Nolen-Hoeksema, S. (2003). Rumination reconsidered: A psychometric analysis. Cognitive Therapy and Research, 27, 247259.

Twenge, J. M., \& Nolen-Hoeksema, S. (2002). Age, gender, race, socioeconomic status, and birth cohort differences on the Children's Depression Inventory: A meta-analysis. Journal of Abnormal Psychology, 111, 578-588.

Unger, R., \& Crawford, M. (1992). Women and gender: A feminist psychology. New York: McGraw-Hill Inc.

Wänke, M., \& Schmid, J. (1996). Rumination: When all else fails. In R. S. Wyer, Jr. (Ed.), Ruminative Thoughts: Advances in Social Cognition, Volume IX (pp. 177187). New Jersey: Lawrence Erlbaum Associates.

Watson, P. D. (2003). A health profile of New Zealand youth who attend secondary school (Electronic Version). Journal of the New Zealand Medical Association, 116.

Weir, K. F., \& Jose, P. E. (August, 2004). The mediating role of anxiety in the relationship between rumination and depression. Paper presented at the New Zealand Psychological Society Conference, Wellington, NZ.

Weir, K. F., \& Jose, P. E. (2006a). The Shapiro Control Inventory: A Downward Extension to Pre and Early Adolescents. Manuscript submitted for publication. 
Weir, K. F., \& Jose, P. E. (2006b). Social influences in developing a ruminative response style. Unpublished manuscript.

Weir, K. F., Jose, P. E., \& Pilcher, E. (2006). Examining the relationship between rumination and depression: Testing the response style theory. Manuscript submitted for publication.

Weissman, M. M., Bland, R. C., Joyce, P. R., Newman, S., Wells, J. E., Wittchen, H. (1993). Sex differences in rates of depression: cross-national perspectives. Journal of Affective Disorders, 29, 77-84.

Weissman, M. M., \& Klerman, G. L. (1977). Sex differences and the epidemiology of depression. Archives of General Psychiatry, 34, 98-111.

Wichstrøm, L. (1999). The emergence of gender difference in depressed mood during adolescence: The role of intensified gender socialisation. Developmental Psychology, 35, 232-245.

Winnicott, D. W. (1965). The maturational processes and the facilitating environment. New York: International Universities Press.

Wood, W., Conway, M., Pushkar, D., \& Dugas, M. J. (2005). People’s perceptions of women's and men's worry about life issues: Worrying about love, accomplishment, or worry? Sex Roles, 53, 545-551.

World Health Organisation (2007). Retrieved August 15, 2007, from http://www.who.int/mental_health/management/depression/definition/e $\mathrm{n} /$

Ziegert, D. I., \& Kistner, J.A. (2002). Response styles theory: Downward extension to children. Journal of Clinical Child and Adolescent Psychology, 31, 325-334. 


\section{Appendix A: Global Stress Scale}

We are interested in how many problems you have in your daily life. Please circle the number that best represents how many problems you have at the moment. Remember there are no right or wrong answers, so please answer as honestly as you can

\begin{tabular}{lccccc}
\hline & $\begin{array}{c}\text { Not } \\
\text { true }\end{array}$ & $\begin{array}{c}\text { A little } \\
\text { true }\end{array}$ & $\begin{array}{c}\text { Somewhat } \\
\text { true }\end{array}$ & $\begin{array}{c}\text { Fairly } \\
\text { true }\end{array}$ & $\begin{array}{c}\text { Very } \\
\text { true }\end{array}$ \\
\hline 1. I have a lot of problems in my life. & 1 & 2 & 3 & 4 & 5 \\
2. I feel stressed a lot of the time. & 1 & 2 & 3 & 4 & 5 \\
3. I have problems that other children don't & 1 & 2 & 3 & 4 & 5 \\
4. I have problems than overwhelm me. & 1 & 2 & 3 & 4 & 5 \\
5. Other children have fewer problems than me. & 1 & 2 & 3 & 4 & 5 \\
6. I feel that I have a lot of big problems. & 1 & 2 & 3 & 4 & 5 \\
\hline
\end{tabular}




\section{Appendix B: Overall Sense of Control Scale}

I am interested in how much control you think you have over your life in general. By control I mean how much influence you have over the events and people in your life. For each sentence, please circle the number that best represents what you think. I am interested in how much control you actually think you have, NOT how much control you think you should have.

\begin{tabular}{|c|c|c|c|c|c|}
\hline & $\begin{array}{r}\text { Strongly } \\
\text { disagree }\end{array}$ & & Neutral & & $\begin{array}{c}\text { Strongly } \\
\text { agree }\end{array}$ \\
\hline 1. I am in control of my life. & 1 & 2 & 3 & 4 & 5 \\
\hline $\begin{array}{l}\text { 2. If I decide to, I can make changes to get more } \\
\text { control over my life. }\end{array}$ & 1 & 2 & 3 & 4 & 5 \\
\hline 3. I am able to confidently change what I want. & 1 & 2 & 3 & 4 & 5 \\
\hline $\begin{array}{l}\text { 4. I am able to make decisions about important things } \\
\text { in my life. }\end{array}$ & 1 & 2 & 3 & 4 & 5 \\
\hline 5. I am able to set clear goals. & 1 & 2 & 3 & 4 & 5 \\
\hline $\begin{array}{l}\text { 6. I am aware of my own feelings and understand how } \\
\text { they affect me. }\end{array}$ & 1 & 2 & 3 & 4 & 5 \\
\hline 7. I make enough effort to reach my goals. & 1 & 2 & 3 & 4 & 5 \\
\hline 8. I have the skills and abilities to reach my goals. & 1 & 2 & 3 & 4 & 5 \\
\hline 9. I have the right amount of self-control. & 1 & 2 & 3 & 4 & 5 \\
\hline 10. I lose control of myself. & 1 & 2 & 3 & 4 & 5 \\
\hline 11. Others have too much control over me. & 1 & 2 & 3 & 4 & 5 \\
\hline 12. I have less control than I used to. & 1 & 2 & 3 & 4 & 5 \\
\hline 13. I lack control over my everyday life. & 1 & 2 & 3 & 4 & 5 \\
\hline 14. I cannot make important decisions about my life. & 1 & 2 & 3 & 4 & 5 \\
\hline $\begin{array}{l}\text { 15. I cannot make changes, to get more control over my } \\
\text { life. }\end{array}$ & 1 & 2 & 3 & 4 & 5 \\
\hline 16. I lack control of my feelings. & 1 & 2 & 3 & 4 & 5 \\
\hline 17. I cannot set clear goals for myself. & 1 & 2 & 3 & 4 & 5 \\
\hline
\end{tabular}




\section{Appendix C: Ruminative Response Styles Questionnaire}

I am interested in how you usually respond to upsetting situations. Please circle the number that best describes you. Remember there are no right or wrong answers.

\begin{tabular}{|c|c|c|c|c|c|}
\hline & Never & & Sometimes & & Always \\
\hline $\begin{array}{l}1 * \text { I think, "There must be something wrong with me } \\
\text { or I wouldn't feel this way." }\end{array}$ & 1 & 2 & 3 & 4 & 5 \\
\hline 2*. I think, "Why can’t I handle things better?" & 1 & 2 & 3 & 4 & 5 \\
\hline 3*. I think, "Why do I always react this way?" & 1 & 2 & 3 & 4 & 5 \\
\hline $\begin{array}{l}\text { 4*. I think, "No one will want to be around me if I don't } \\
\text { snap out of this mood." }\end{array}$ & 1 & 2 & 3 & 4 & 5 \\
\hline $\begin{array}{l}\text { 5*. I think, "Why can't I be satisfied with the way things } \\
\text { are?" }\end{array}$ & 1 & 2 & 3 & 4 & 5 \\
\hline $\begin{array}{l}\text { 6*. I think, "I must have serious problems, otherwise I } \\
\text { wouldn't feel this way." }\end{array}$ & 1 & 2 & 3 & 4 & 5 \\
\hline 7*. I think, "I need to understand these feelings." & 1 & 2 & 3 & 4 & 5 \\
\hline 8*. I think, "Why can't I get going?" & 1 & 2 & 3 & 4 & 5 \\
\hline $\begin{array}{l}\text { 9*. I think, "Why do I have problems that other people } \\
\text { don't seem to have." }\end{array}$ & 1 & 2 & 3 & 4 & 5 \\
\hline $\begin{array}{l}\text { 10*. I think, "I won't be able to concentrate if I keep } \\
\text { feeling this way." }\end{array}$ & 1 & 2 & 3 & 4 & 5 \\
\hline 11. I go to my room alone to think about my feelings. & 1 & 2 & 3 & 4 & 5 \\
\hline 12. I sit at home and think about how I feel. & 1 & 2 & 3 & 4 & 5 \\
\hline 13. I listen to sad music. & 1 & 2 & 3 & 4 & 5 \\
\hline $\begin{array}{l}\text { 14. I isolate myself and think about the reasons I'm } \\
\text { feeling this way. }\end{array}$ & 1 & 2 & 3 & 4 & 5 \\
\hline 15. I write about my feelings (i.e. journal/diary/letter). & 1 & 2 & 3 & 4 & 5 \\
\hline 16. I talk to others about how I'm feeling. & 1 & 2 & 3 & 4 & 5 \\
\hline
\end{tabular}

Note: $*=$ items used in the shortened version of the scale described in Chapter 2. 


\section{Appendix D: The Children's Depression Inventory}

For each number below, pick one sentence out of the three statements that best describes how you have felt for the past two weeks (by ticking on the line). Remember, there are no right or wrong answers.

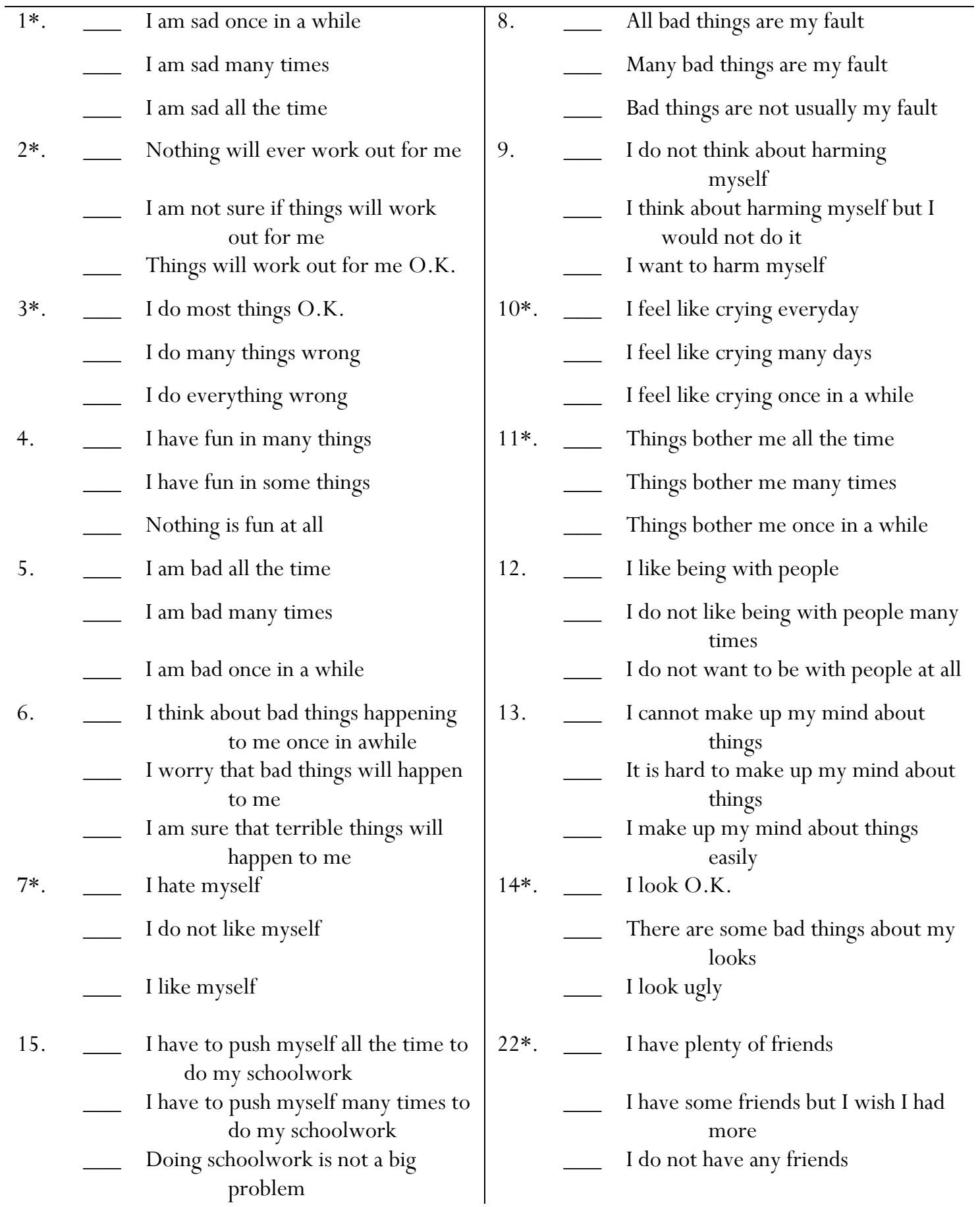


16.

__ I have trouble sleeping every night

I have trouble sleeping many nights

I sleep pretty well

17.

I am tired once in a while

I am tired many days

_ I am tired all of the time

18.

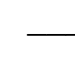

_ I eat pretty well

19.

_ I do not feel alone

$$
\text { worry about aches and }
$$$$
\text { pains }
$$

__ I worry about aches and pains many times

_ I worry about aches and pains all the time

I feel alone many times

I feel alone all the time

21. __ I never have fun at school

I have fun at school only once in a while

I have fun at school many times
23.

_ My school work is alright

_ My school work is not as good as before

I do very badly in subjects I used to be good in

I can never be as good as other people

I can be as good as other people if I want to

I am just as good as other people

25*. _ _ Nobody really loves me

I am not sure if anybody loves me

I am sure that somebody loves me

26. __ I usually do what I am told

I do not do what I am told most times

I never do what I am told

27.

I get along with people

I get into fights many times

I get into fights all the time

Note: $*=$ items used in the shortened version of the scale described in Chapter 4 (Study 3). 


\section{Appendix E: Stressful Scenarios presented to Preadolescents}

Scenario: Andrew studies hard for a test, but he gets a bad mark.

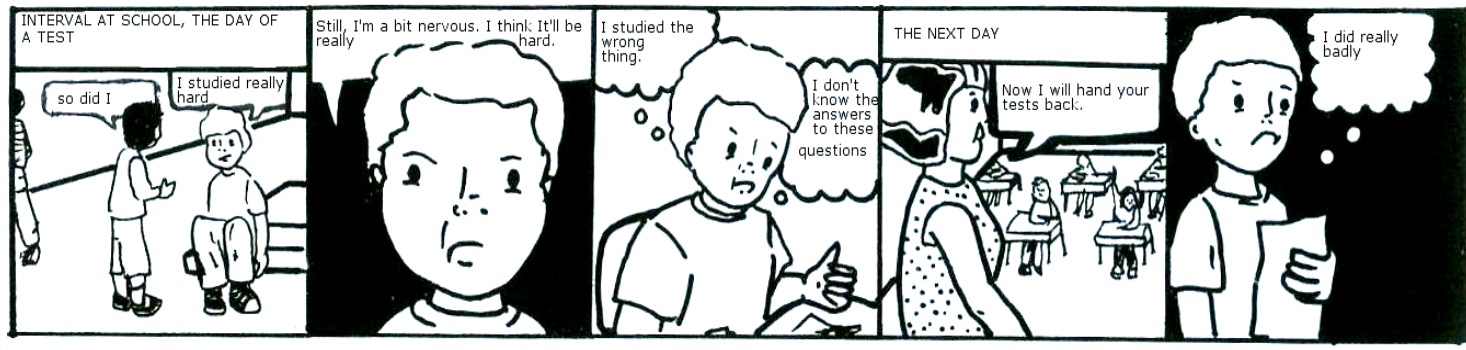

How do you think Andrew will respond to this situation?

$\begin{gathered}\text { Not at A little Some } \\ \text { all }\end{gathered}$
A fair Constantly
amount

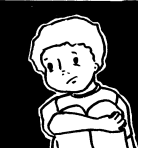

Think to himself, "I won't be able to concentrate if I keep feeling this way."
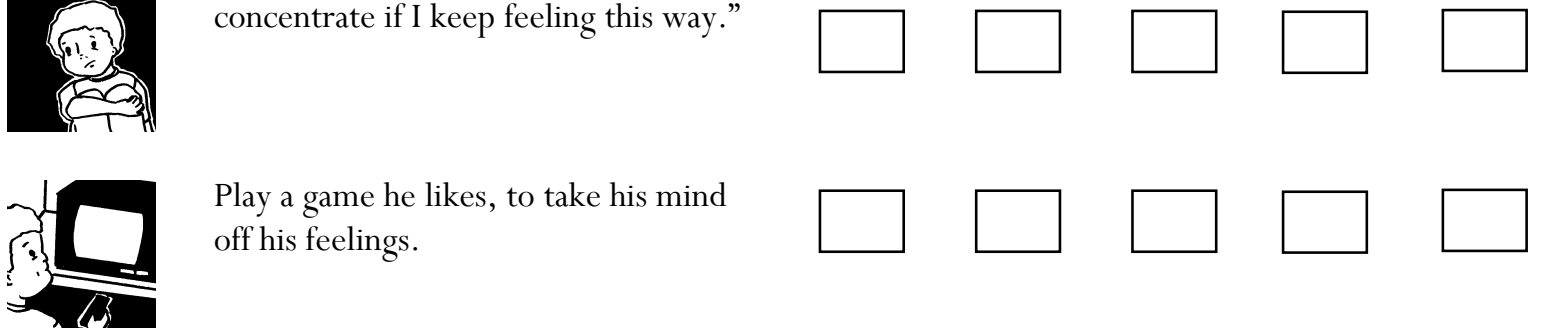

Play a game he likes, to take his mind off his feelings.
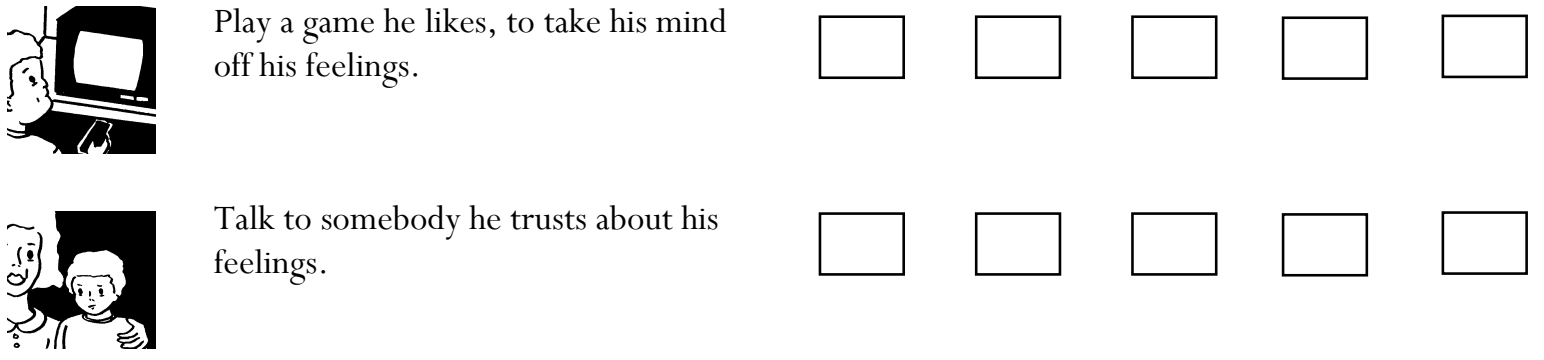

Talk to somebody he trusts about his feelings.
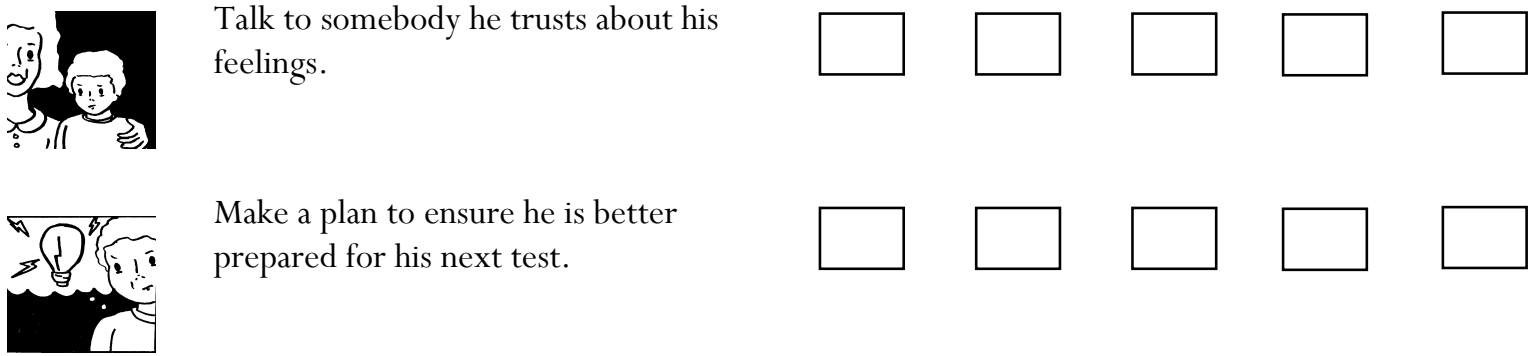

Make a plan to ensure he is better prepared for his next test.
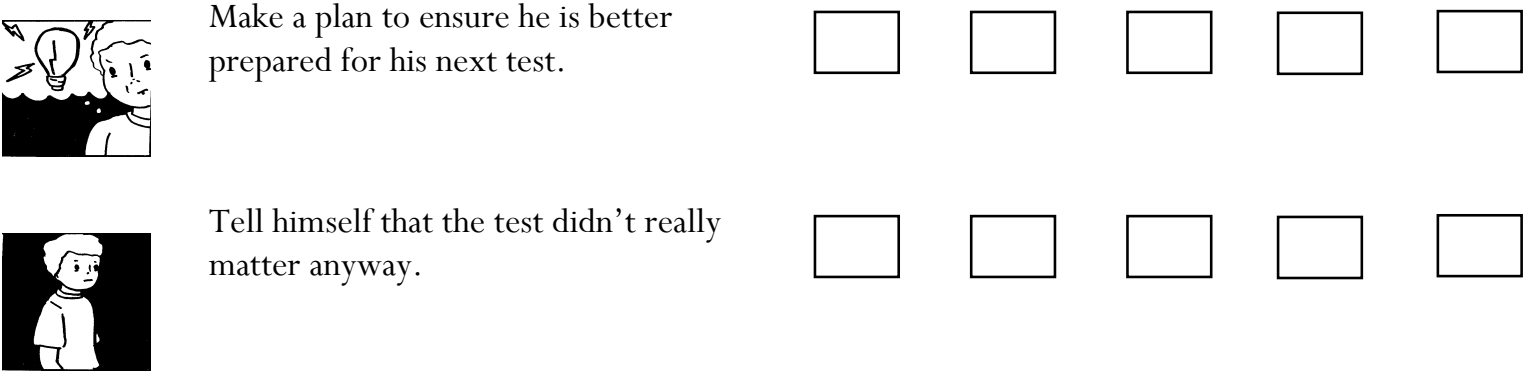

Tell himself that the test didn't really matter anyway.
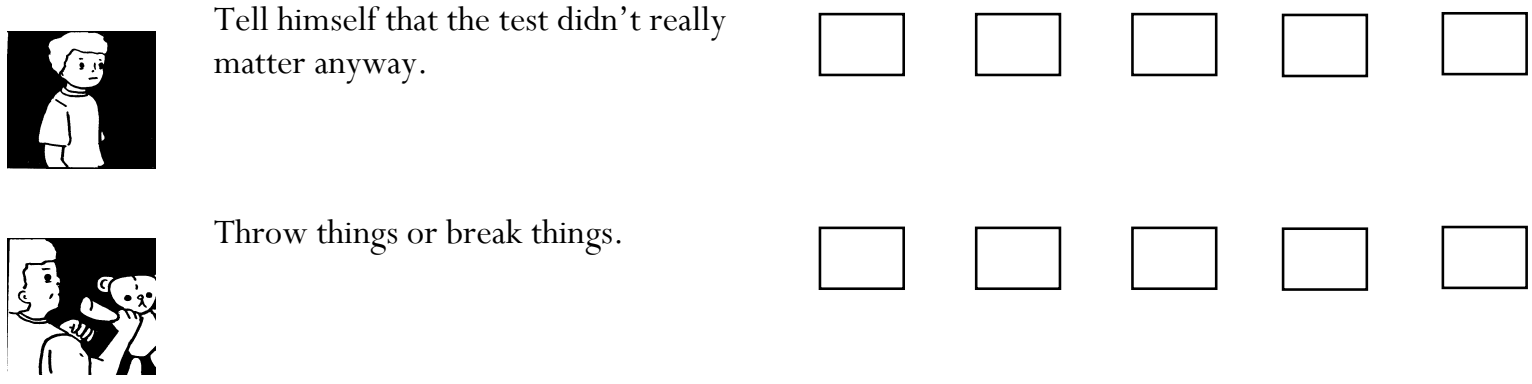

Throw things or break things.
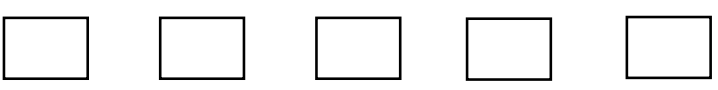
Scenario: Andrew is bored at school by the work.

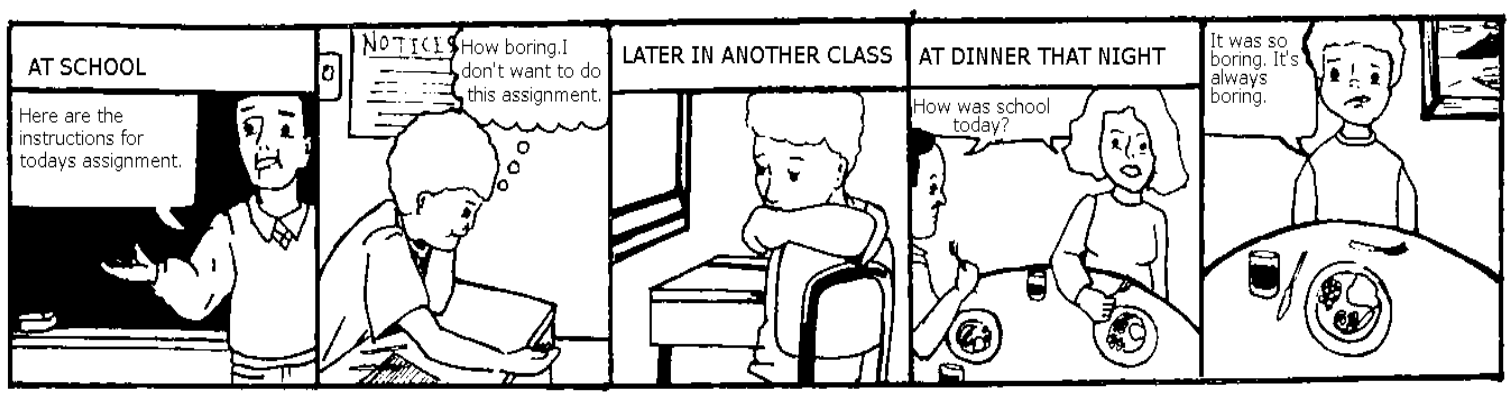

How do you think Andrew will respond to this situation?

$\begin{gathered}\text { Not at A little } \\ \text { all }\end{gathered}$
A fome
amount

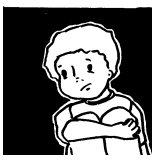

Think to himself, "there must be something wrong with me or I wouldn't react this way."

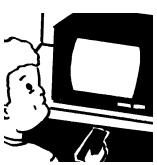

Does something he enjoys to take his mind off school.

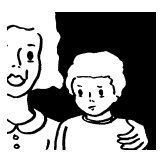

Talk to his parents about how bored he is at school.

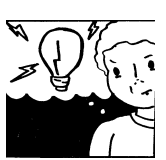

Make a plan about how to make school more interesting.

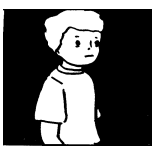

Tell himself that being bored at school isn't really a problem.

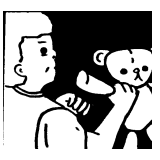

Yell or scream about how boring school is.
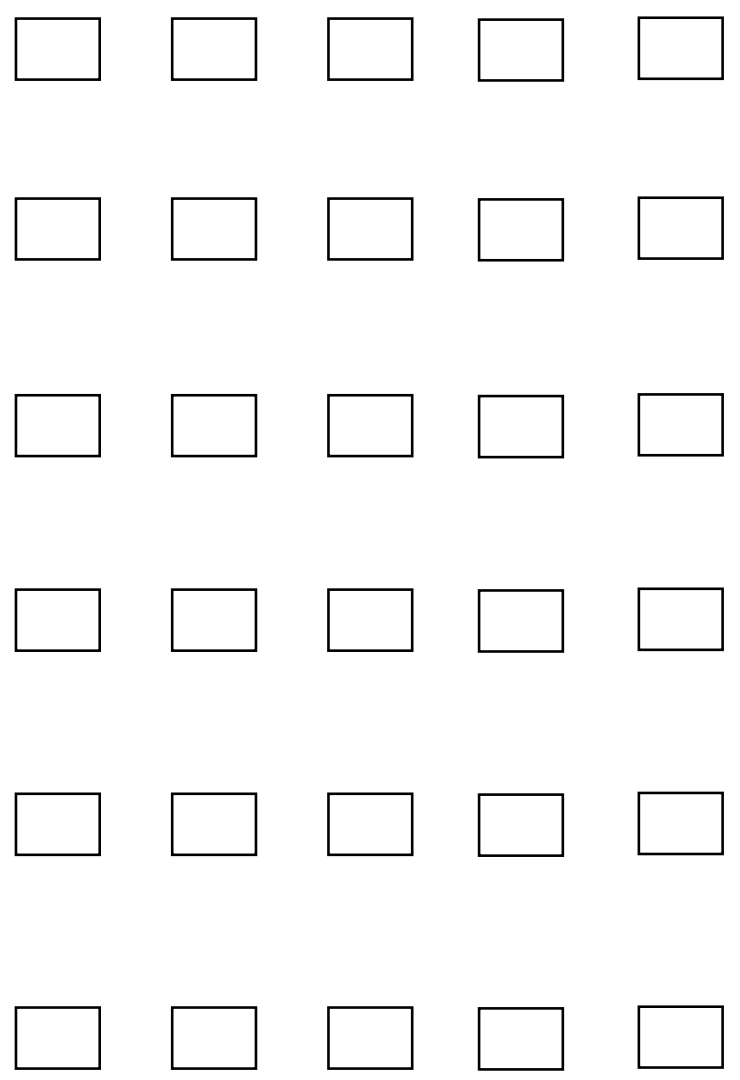
Scenario: Andrew's teacher at school is picking on him.

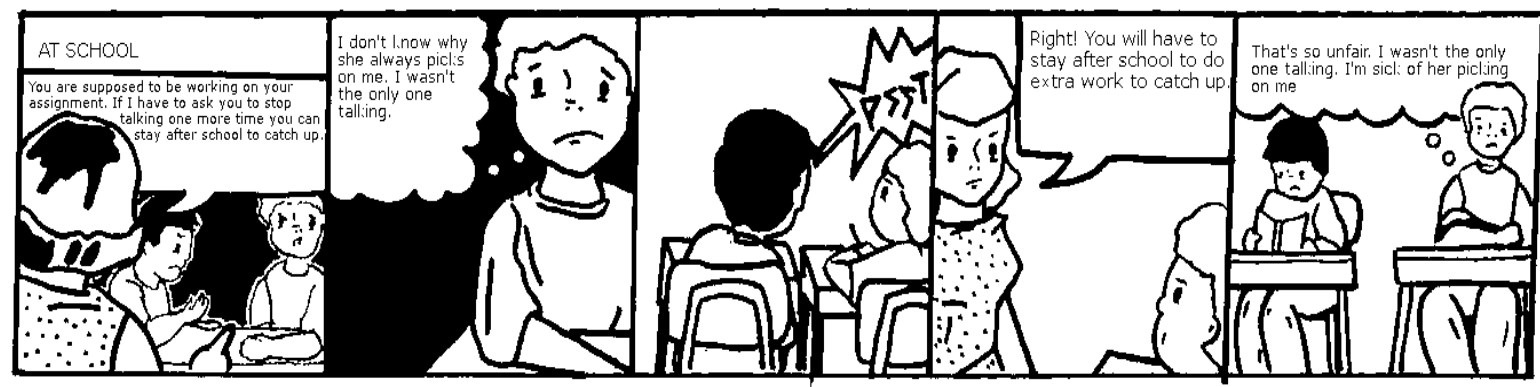

How do you think Andrew will respond to this situation?

$\begin{array}{cccc}\begin{array}{c}\text { Not at A little } \\ \text { all }\end{array} & \text { Some fair } & \text { Constantly } \\ \text { amount }\end{array}$

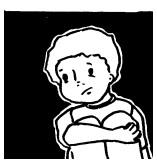

Think to himself, “why can't I handle things better?"

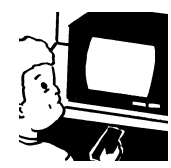

Get on with his schoolwork to take his mind off his feelings.

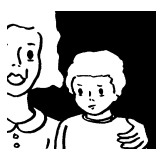

Talk to someone he trusts about how unfair he thinks things are at school.

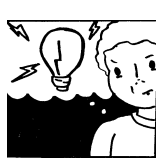

Change something about himself to solve the problem.

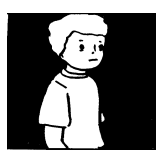

Tell himself it doesn't really matter that his teacher is picking on him.

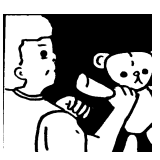

Yell or scream about how unfair things are.
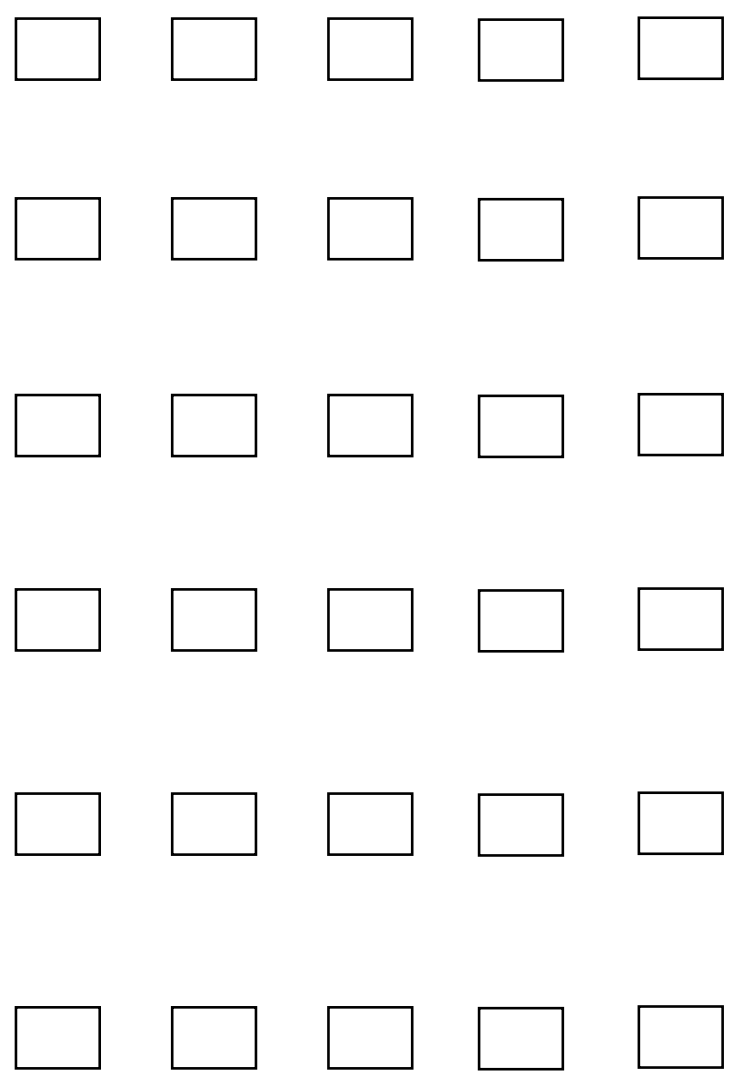
Scenario: Andrew gets blamed by his brother for something that he didn't do.

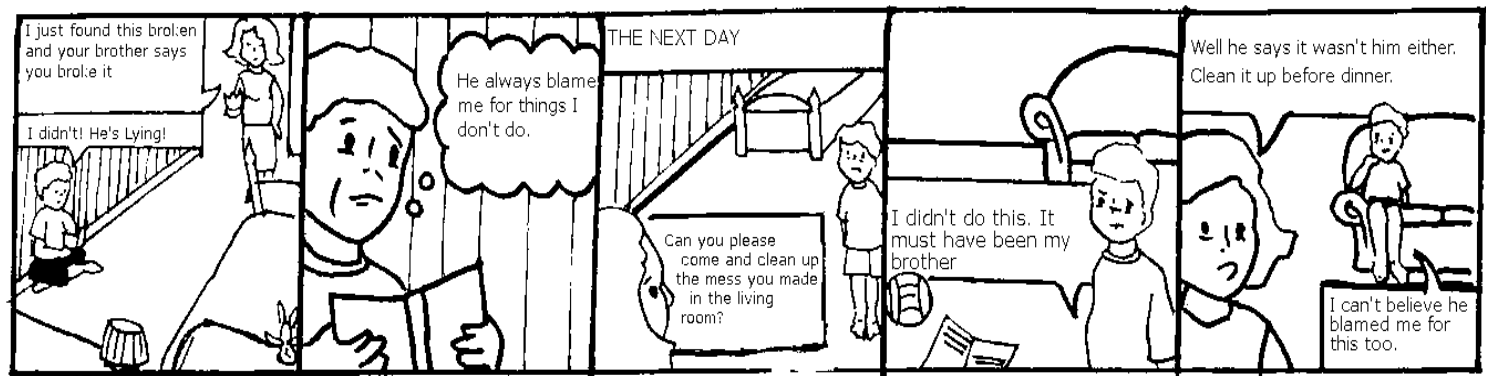

How do you think Andrew will respond?

$\begin{array}{cccc}\begin{array}{c}\text { Not at A little } \\ \text { all }\end{array} & \text { Some fair } & \text { Constantly } \\ \text { amount }\end{array}$

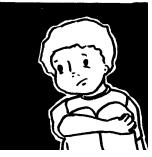

Think to himself, "why do I have problems that other people don't seem to have?"

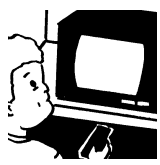

Go back to reading his book to make himself feel better.

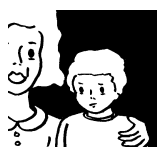

Talk to his mother about how he is feeling.
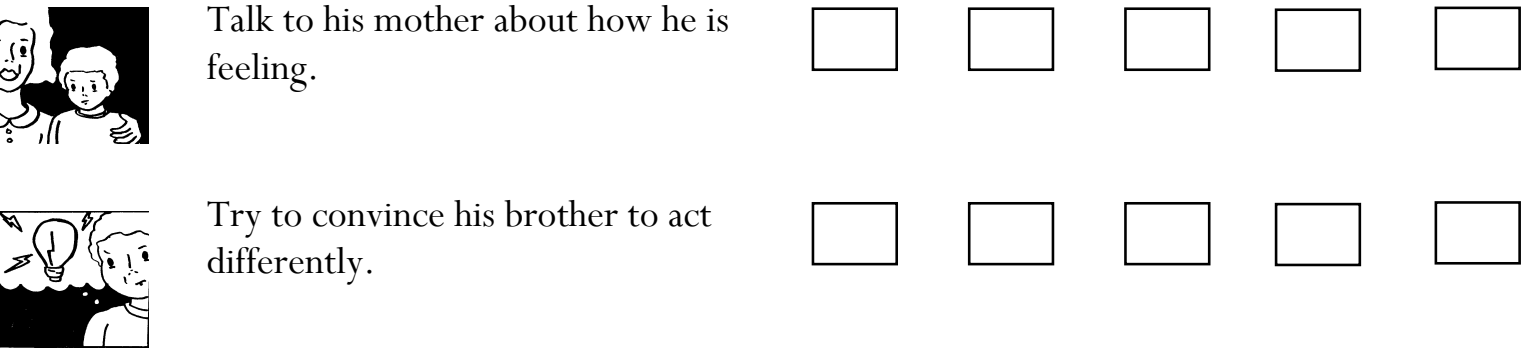

Try to convince his brother to act differently.
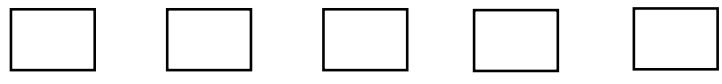

Act like the situation is not a problem.
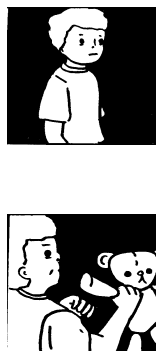

Go back to his room and throw or break things. 
Scenario: Andrew has a fight with his sister over the last piece of cake.

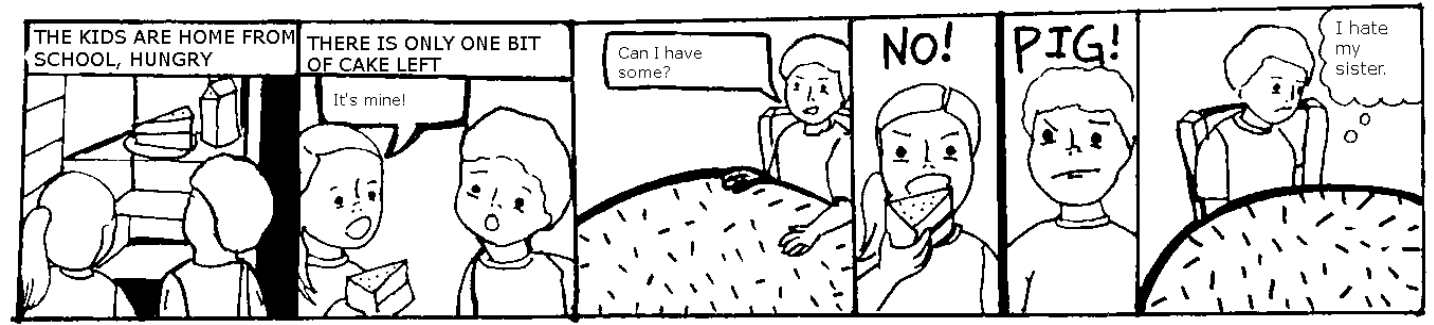

How do you think Andrew will respond to this situation?

$\begin{gathered}\text { Not at A little Some } \\ \text { all }\end{gathered}$
A fair Constantly
amount

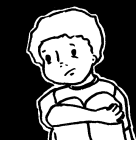

Think to himself, "why can't I

handle things better?"
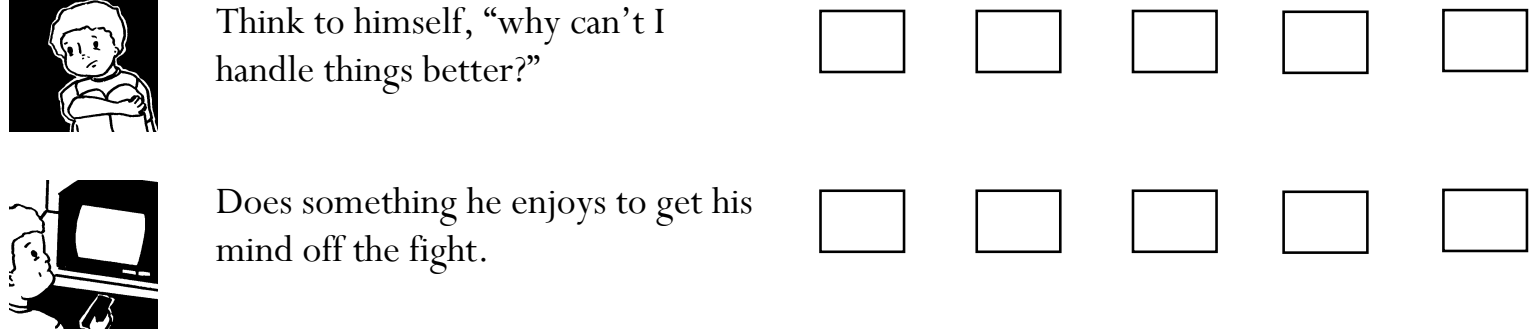

Does something he enjoys to get his mind off the fight.
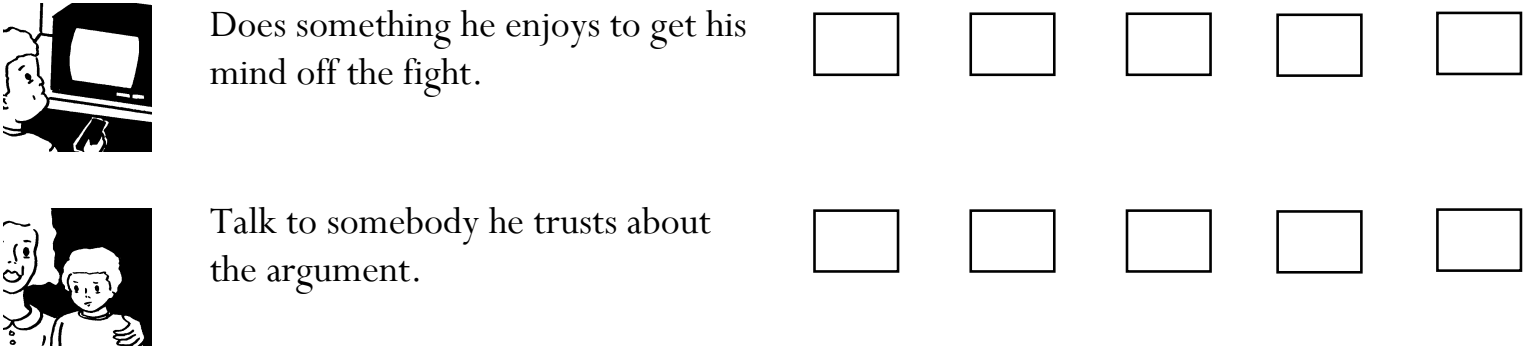

Talk to somebody he trusts about the argument.
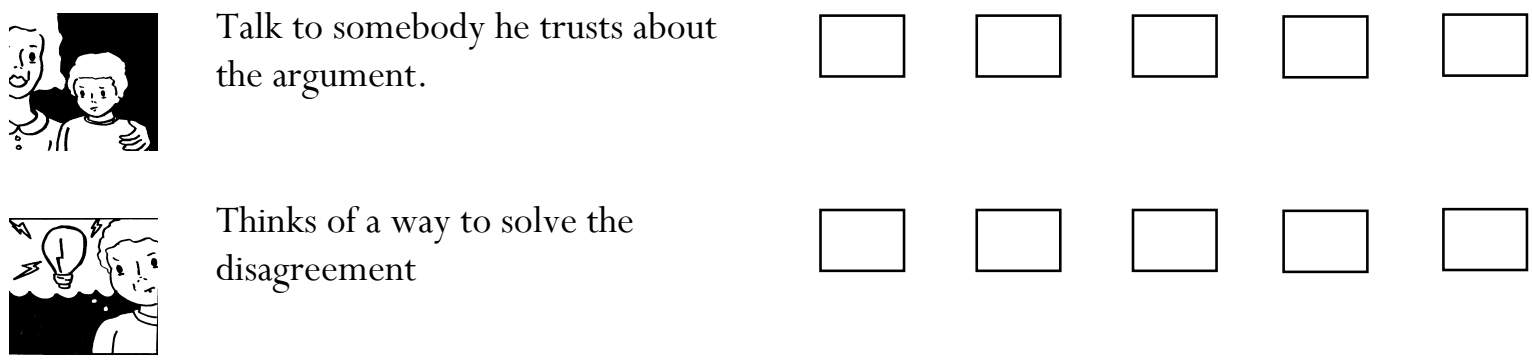

Thinks of a way to solve the disagreement
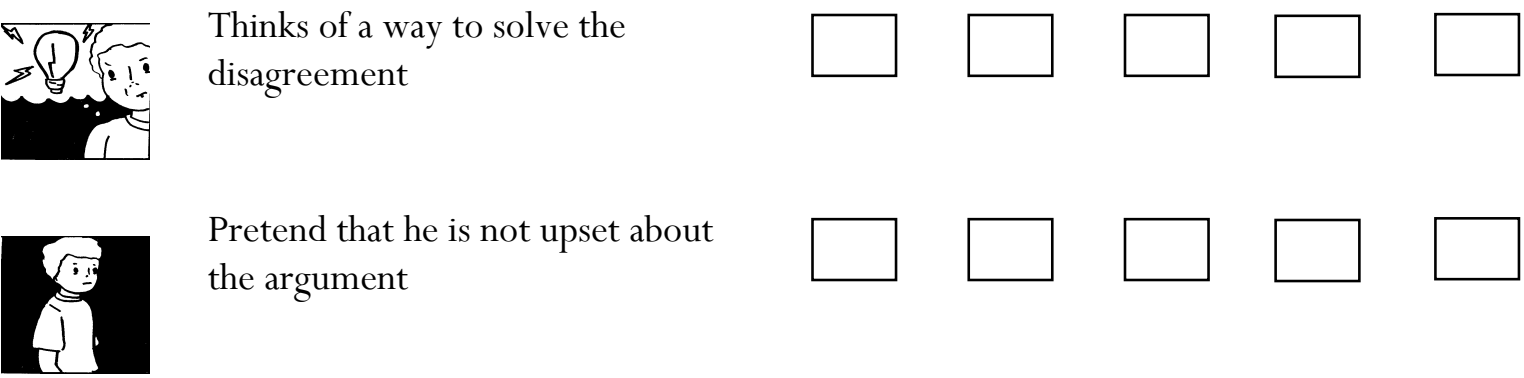

Pretend that he is not upset about the argument
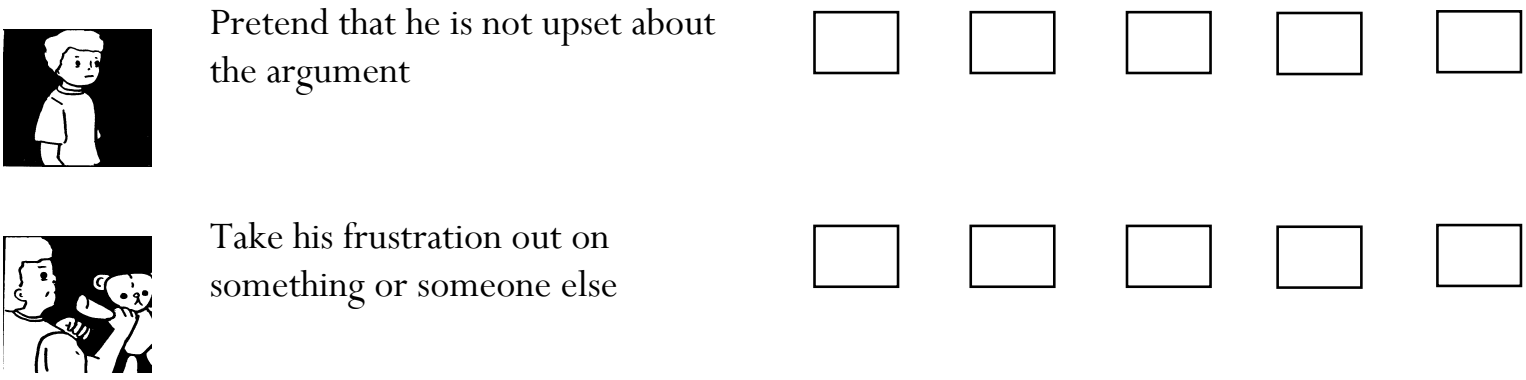

Take his frustration out on something or someone else
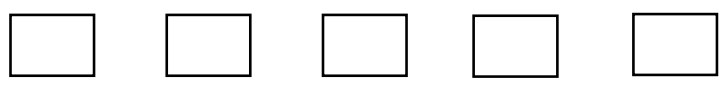
Scenario: Andrew hears his parents arguing because they do not like his friend.

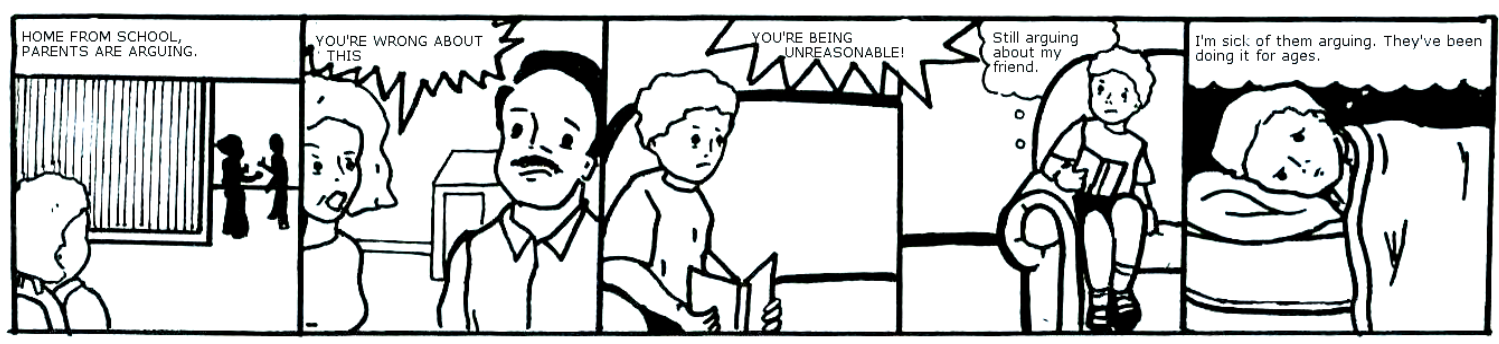

How do you think Andrew will respond to this situation?

Not at A little Some
all $\quad \begin{gathered}\text { A fair } \\ \text { amount }\end{gathered}$ Constantly

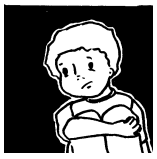

Think to himself, "there must be something wrong with me or I wouldn't react this way.”

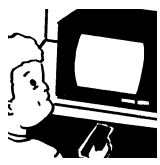

Read a book/watch TV to take his mind off the fighting.
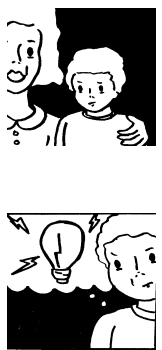

Talk to one of his parents about how he is feeling.

Think of a way to stop the fighting.
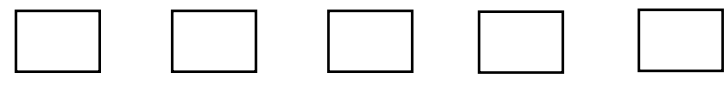

Tell himself he doesn't care what his parents think of his friend.
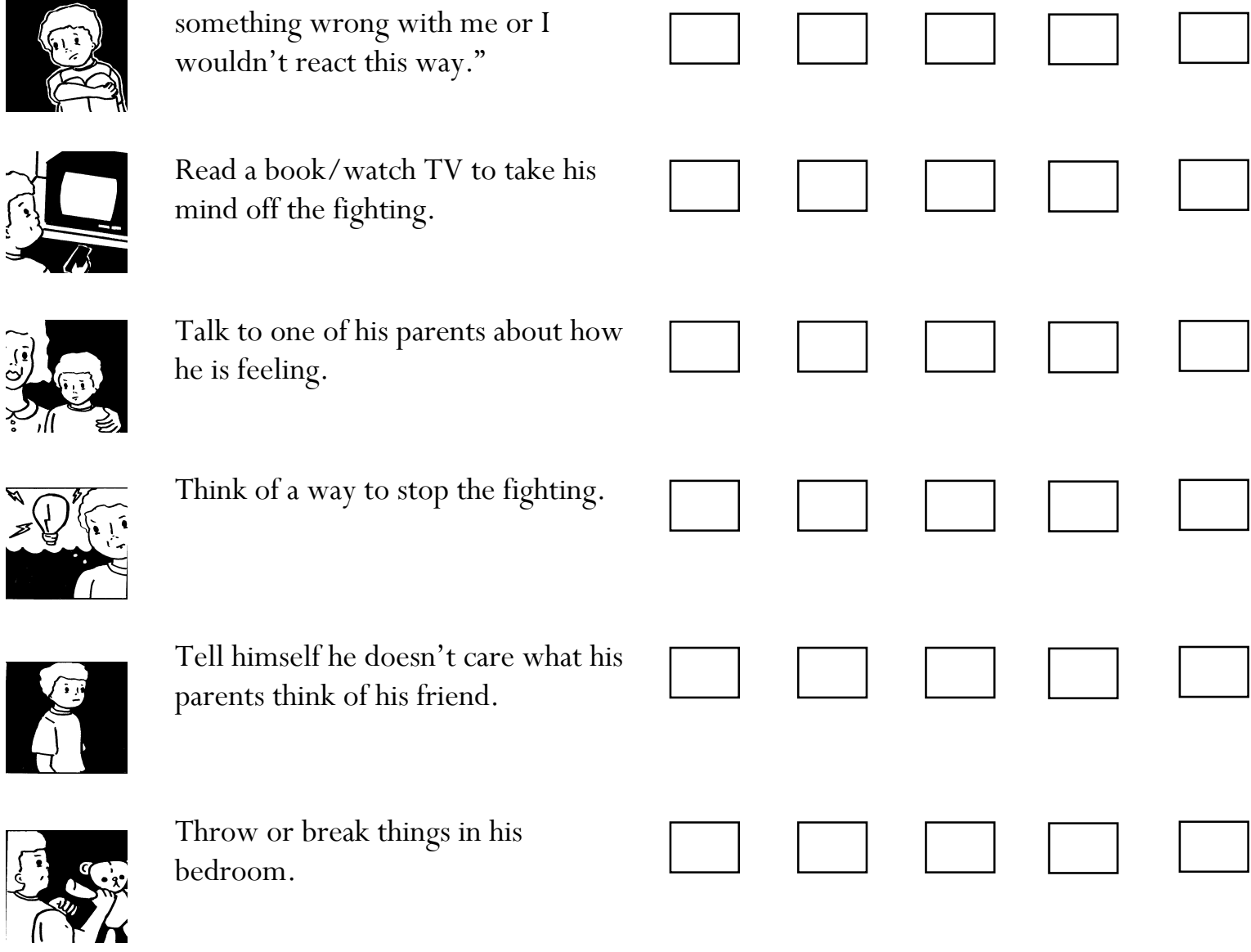
Throw or break things in his
bedroom.
Throw or break things in his
bedroom.
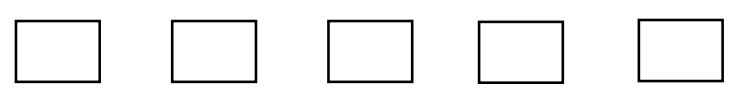

\section{(1)}


Scenario: Andrew doesn't get invited to a birthday party.

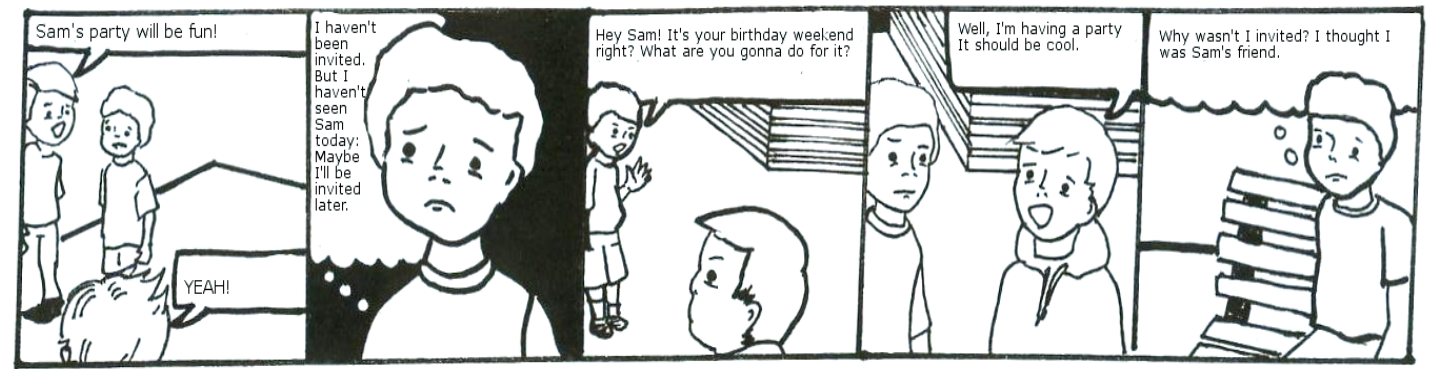

How do you think Andrew will respond to this situation?

$\begin{array}{cccc}\begin{array}{c}\text { Not at A little Some } \\ \text { all }\end{array} & \begin{array}{c}\text { A fair } \\ \text { amount }\end{array} & \text { Constantly }\end{array}$

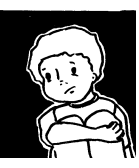

Think to himself, "there must be something wrong with me or I would have been invited.”

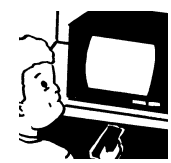

Play a game he enjoys to take his mind off the birthday party.

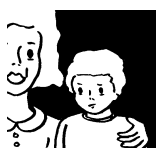

Talk to somebody about not being invited to the party.

Think of a way to deal with his disappointment.
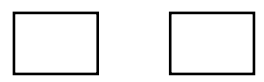

Tell himself that he didn't want to be invited anyway
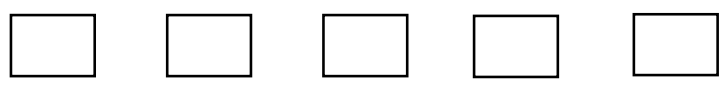
a

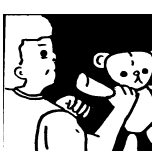

Take his feelings out on somebody else.

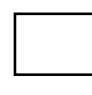

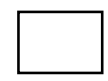
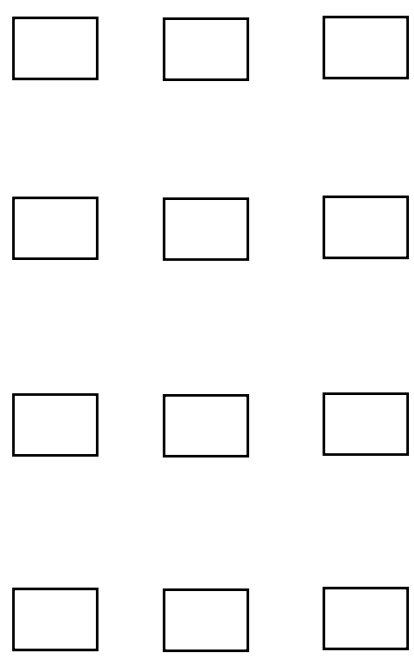

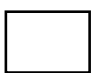


Scenario: Other children are teasing Andrew at school.

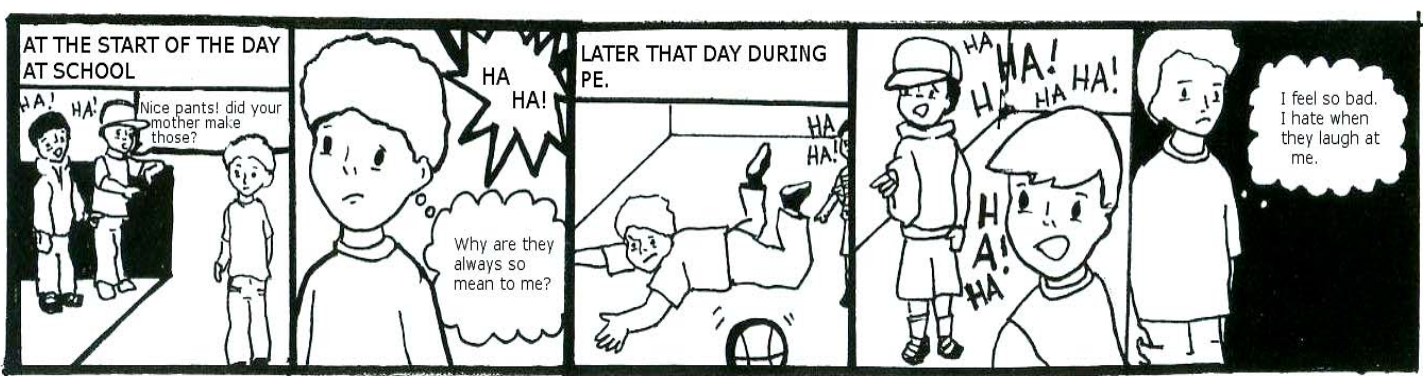

How do you think Andrew will respond to this situation?

$$
\begin{gathered}
\text { Not at A little Some } \\
\text { all }
\end{gathered} \quad \begin{gathered}
\text { A fair } \\
\text { amount }
\end{gathered} \text { Constantly }
$$

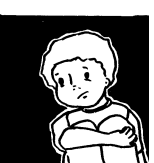

Think to himself, "no one will want to be around me if I don't snap out of this mood.”

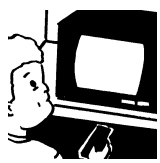

Play a game that he enjoys to take his mind off his feelings.
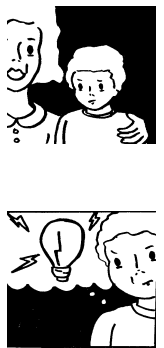

Think of a way to stop the other kids teasing him.

Talk to someone he trusts about the other kids teasing him.
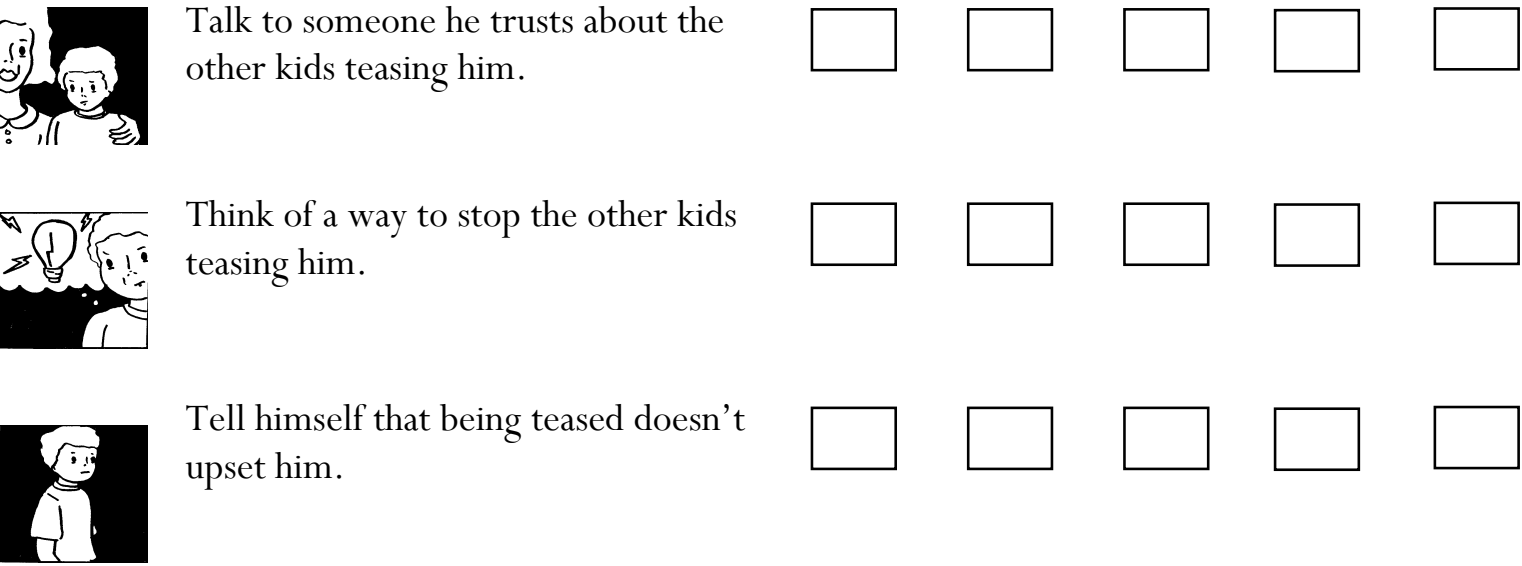

Tell himself that being teased doesn't upset him.

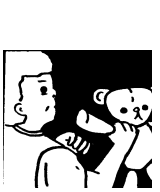

Get into a fight or argument.
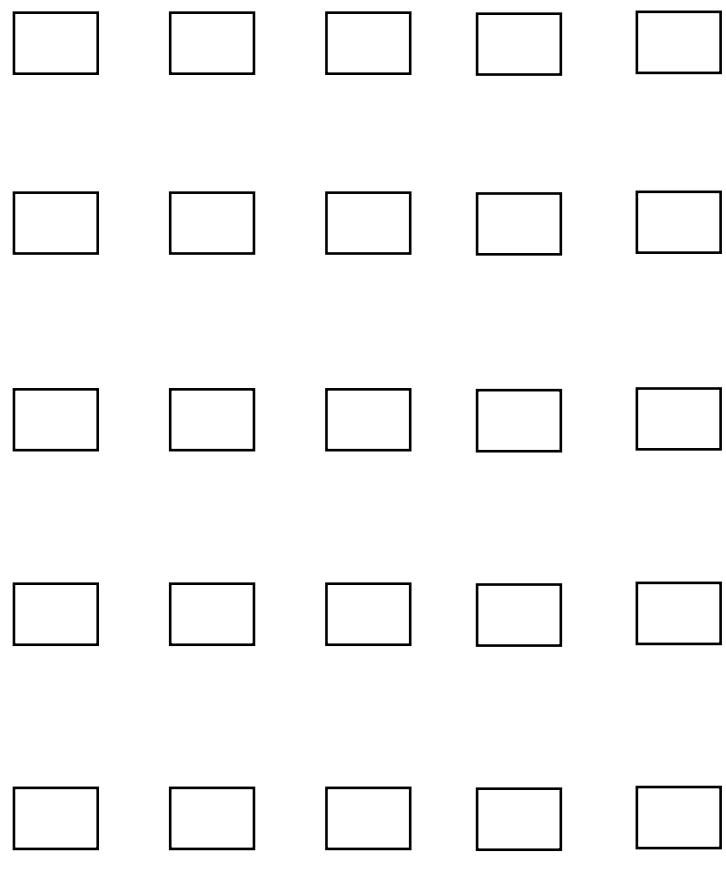

\section{(1)} . 
Scenario: Andrew has a fight with a close friend over what movie they are going to.

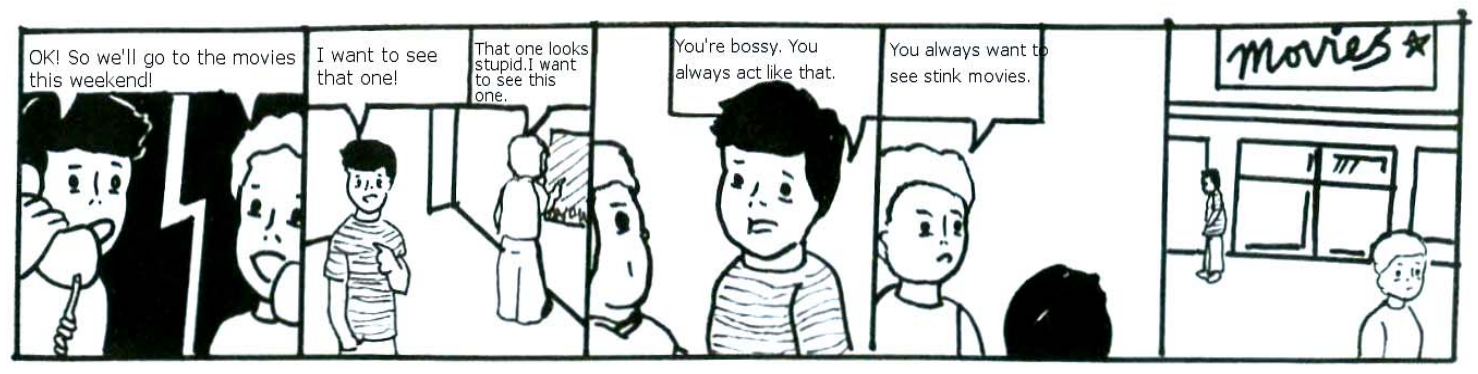

How do you think Andrew will respond to this situation?

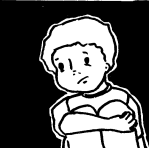

Think, "there must be something wrong with me or we wouldn't have fought."
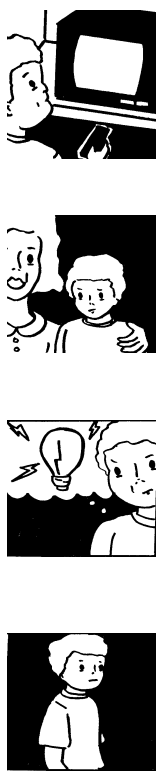

Pretend that he is not upset about the fight.

Talk to another person about how he is feeling.

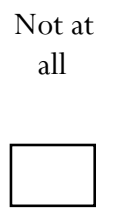

A little

Some

A fair amount

Watch a different movie by himself to take his mind off the argument.
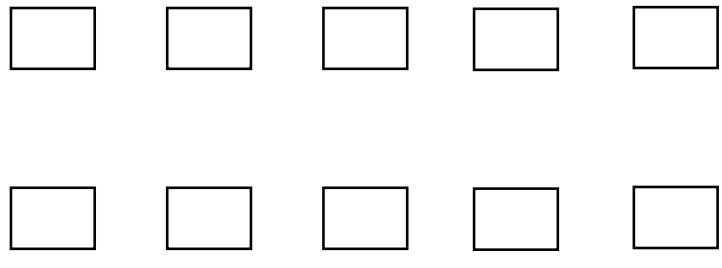

is feeling

Change something about himself to solve the problem.
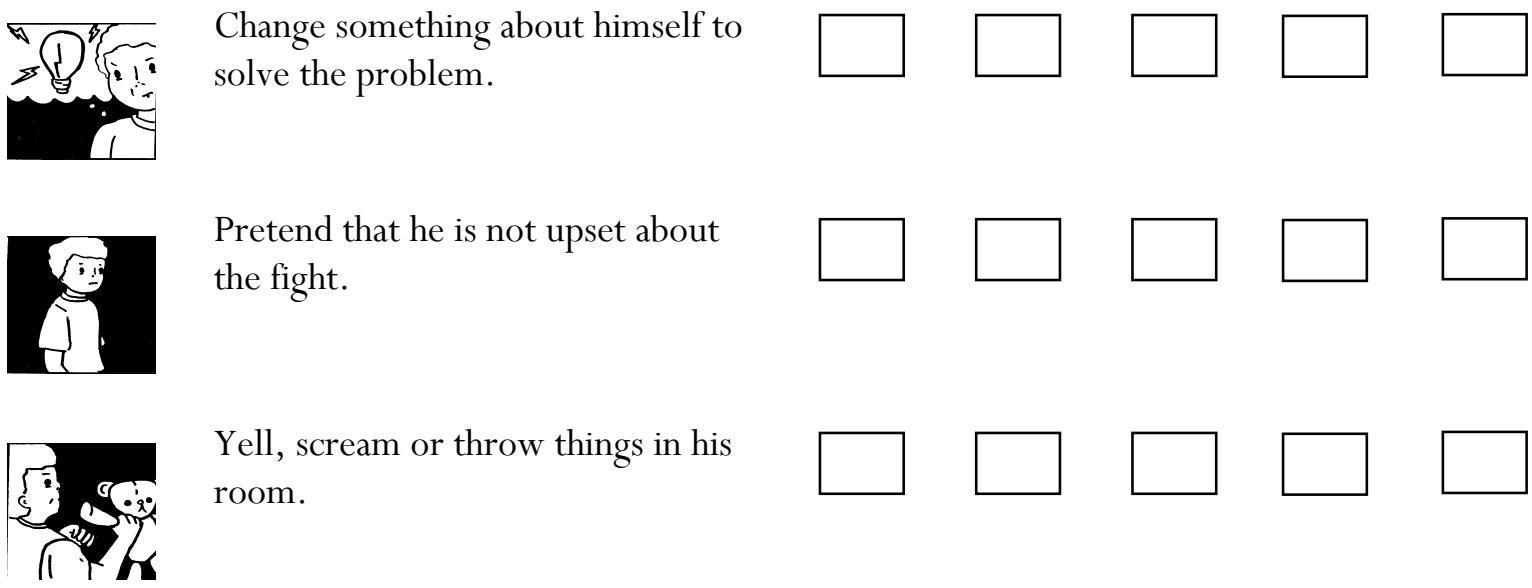

Yell, scream or throw things in his room.
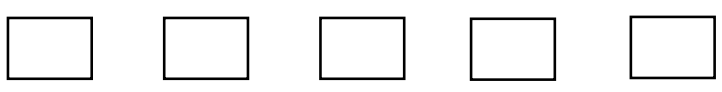


\section{Appendix F: Stressful Scenarios presented to Parents}

Your child has a fight with a close friend.

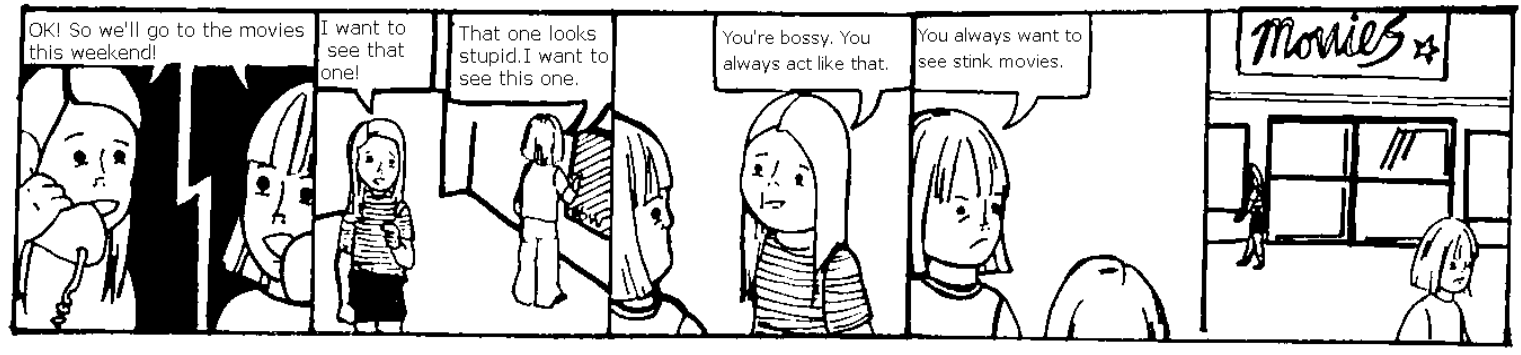

What would you recommend she do?

$\begin{array}{cccc}\begin{array}{c}\text { Strongly } \\ \text { discourage }\end{array} & \begin{array}{c}\text { Somewhat } \\ \text { discourage }\end{array} & \text { Neutral } & \begin{array}{c}\text { Somewhat Strongly } \\ \text { encourage encourage }\end{array}\end{array}$

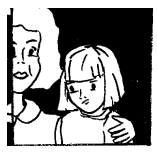

Talk to another person about how she is feeling.
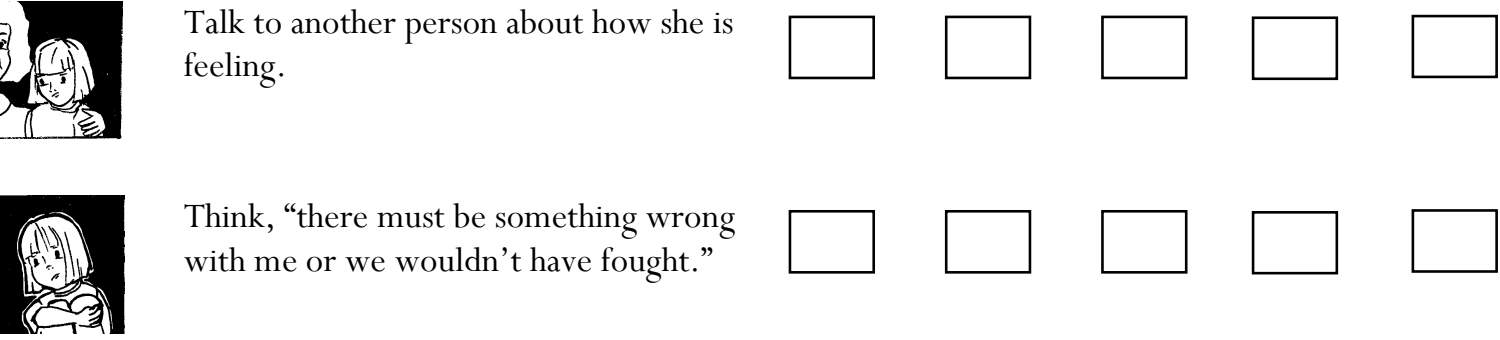

Think, "there must be something wrong with me or we wouldn't have fought.”
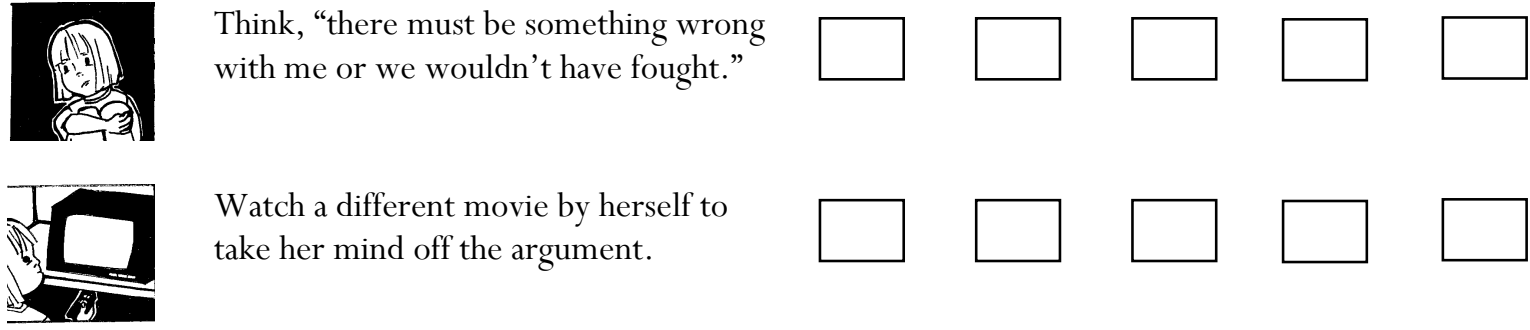

Watch a different movie by herself to take her mind off the argument.
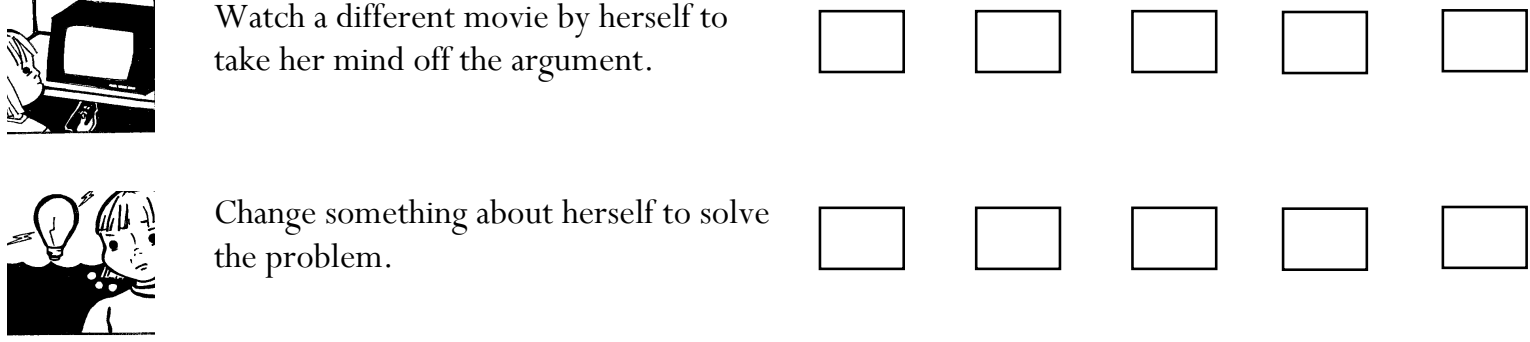

Change something about herself to solve the problem.
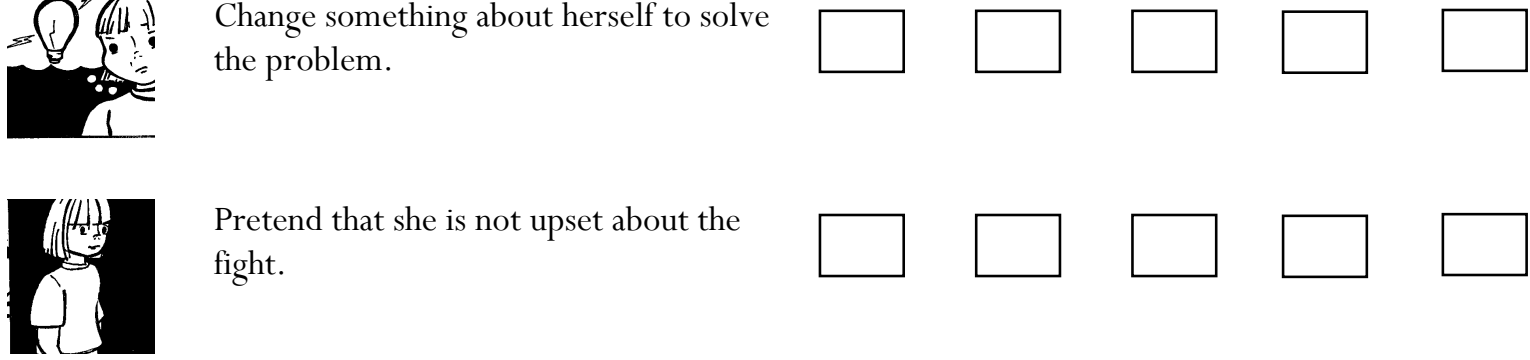

Pretend that she is not upset about the fight.
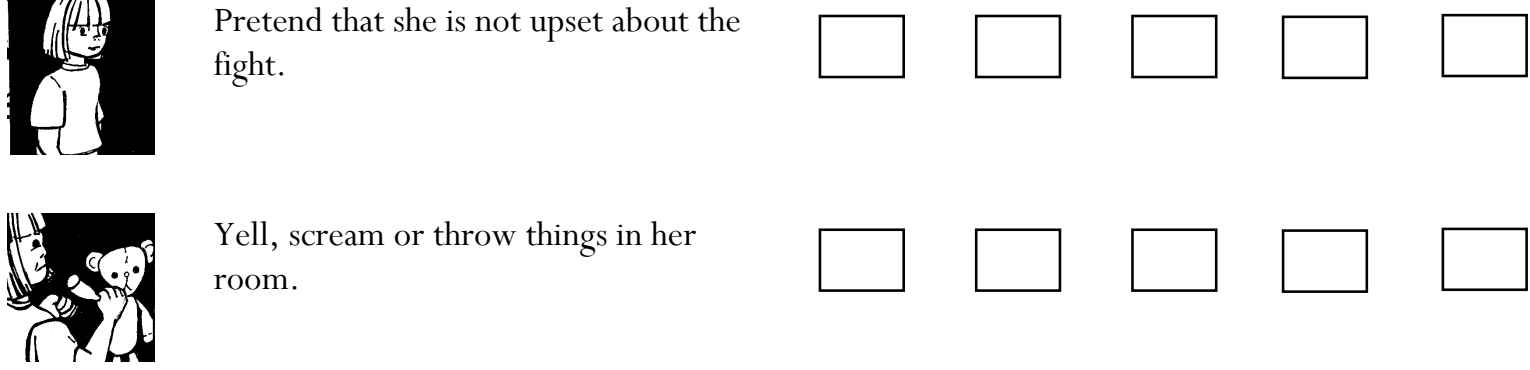

Yell, scream or throw things in her room.
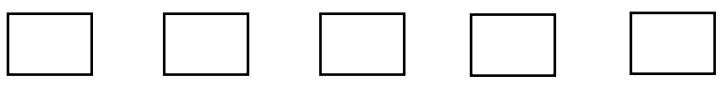
Your child has a fight with their sister.

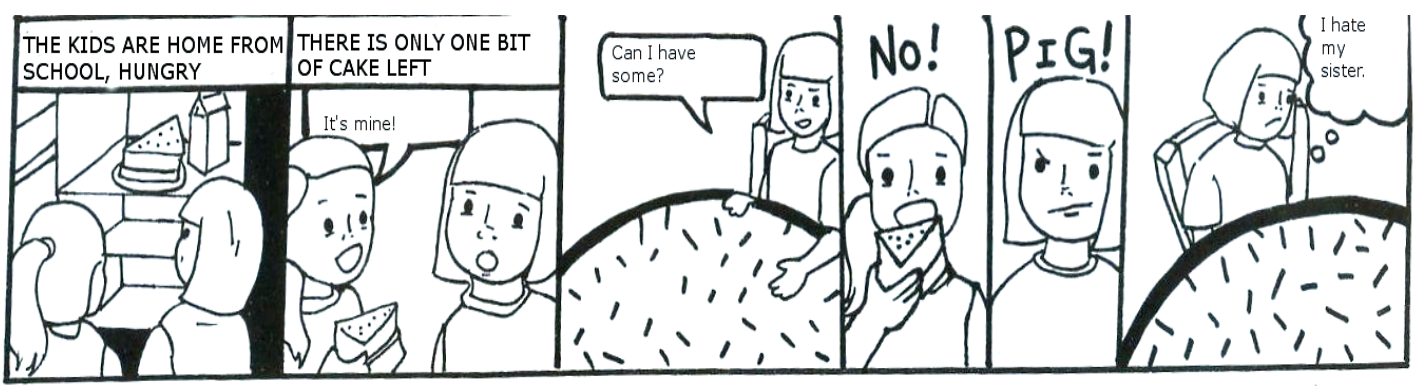

What would you recommend she do?

$\begin{array}{clll}\text { Strongly } & \text { Somewhat } & \text { Neutral } & \begin{array}{l}\text { Somewhat Strongly } \\ \text { encourage encourage }\end{array}\end{array}$

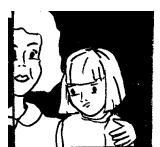

Talk to somebody she trusts about the argument.

Think to herself, “why can't I handle things better?"

Do something she enjoys to get her mind off the fight.

Think of a way to solve the disagreement.

Pretend that she is not upset about the argument.

Take her frustration out on something

or someone else.
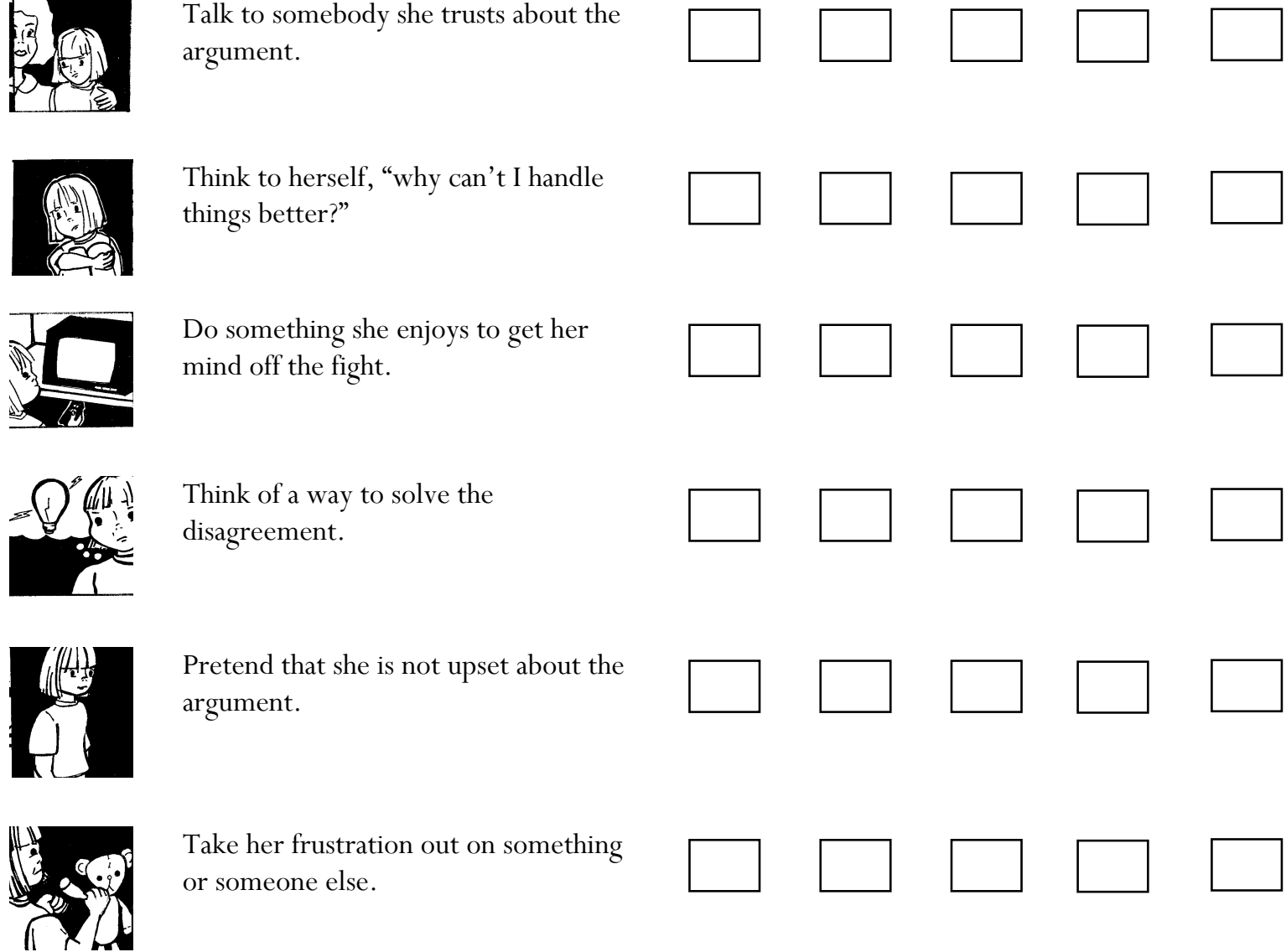
Your child hears you (their parents/guardians) arguing over one of your child's friends.

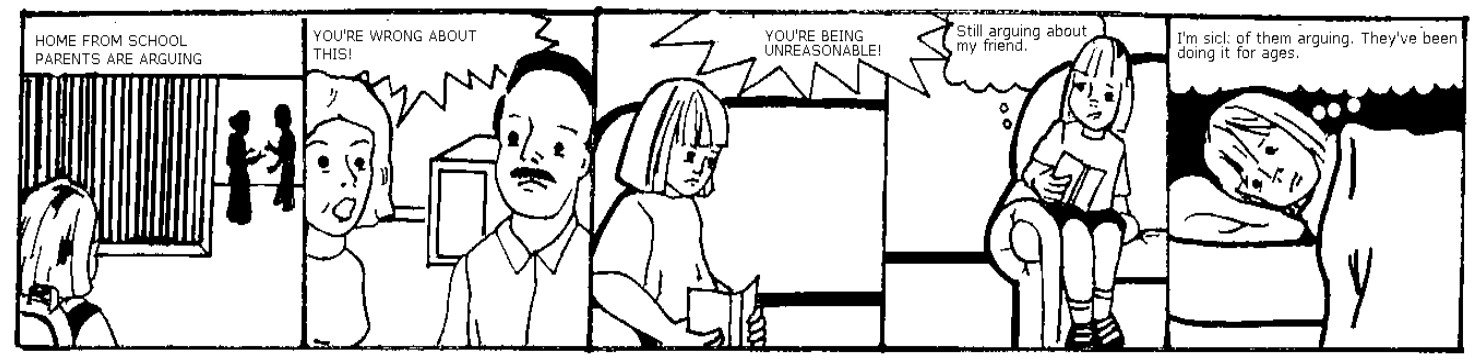

What would you recommend she do?

$\begin{array}{cccc}\begin{array}{c}\text { Strongly } \\ \text { discourage }\end{array} & \begin{array}{c}\text { Somewhat } \\ \text { discourage }\end{array} & \text { Neutral } & \begin{array}{c}\text { Somewhat Strongly } \\ \text { encourage encourage }\end{array}\end{array}$

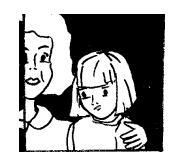

Talk to one of her parents about how she is feeling.
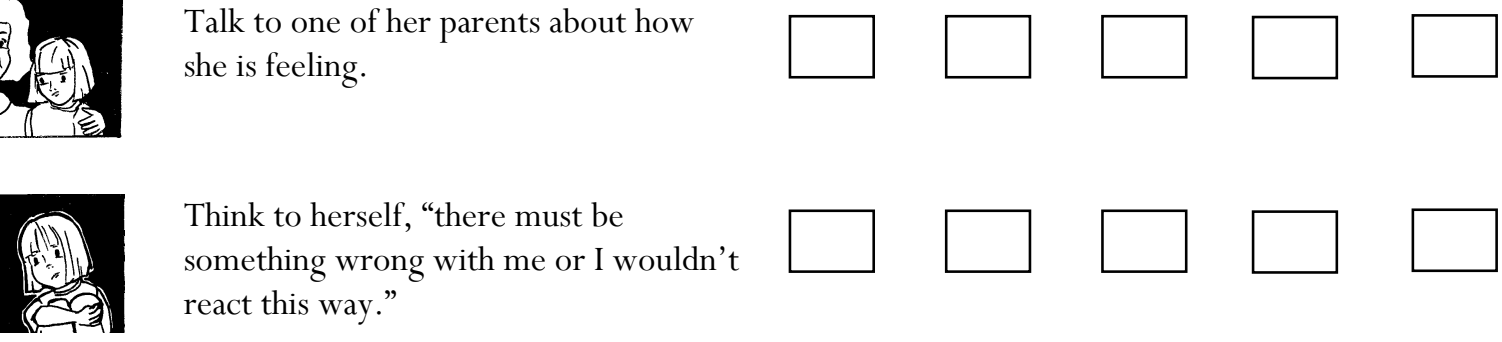

Think to herself, "there must be something wrong with me or I wouldn't react this way."

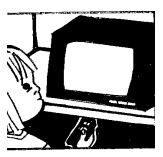

Read a book/watch TV to take her mind off the fighting.
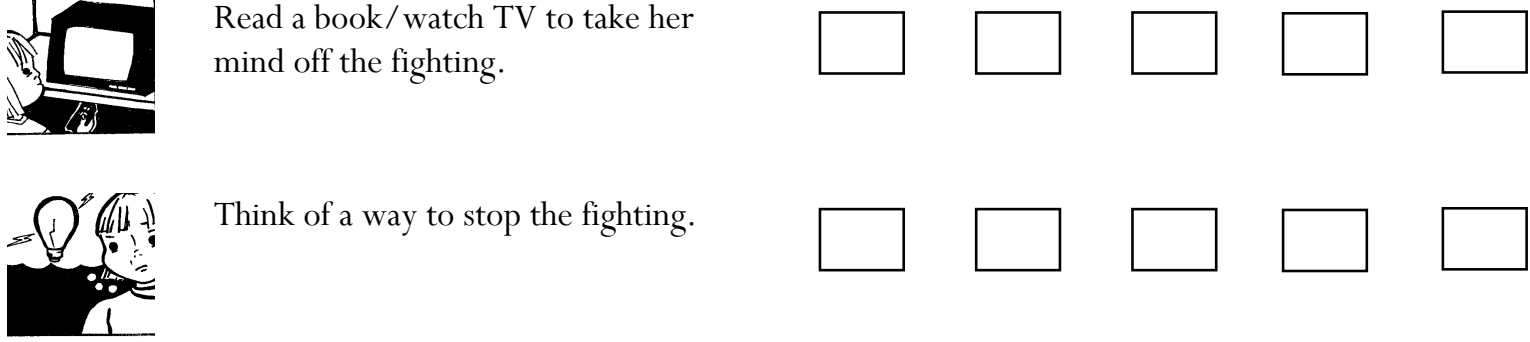

Think of a way to stop the fighting.
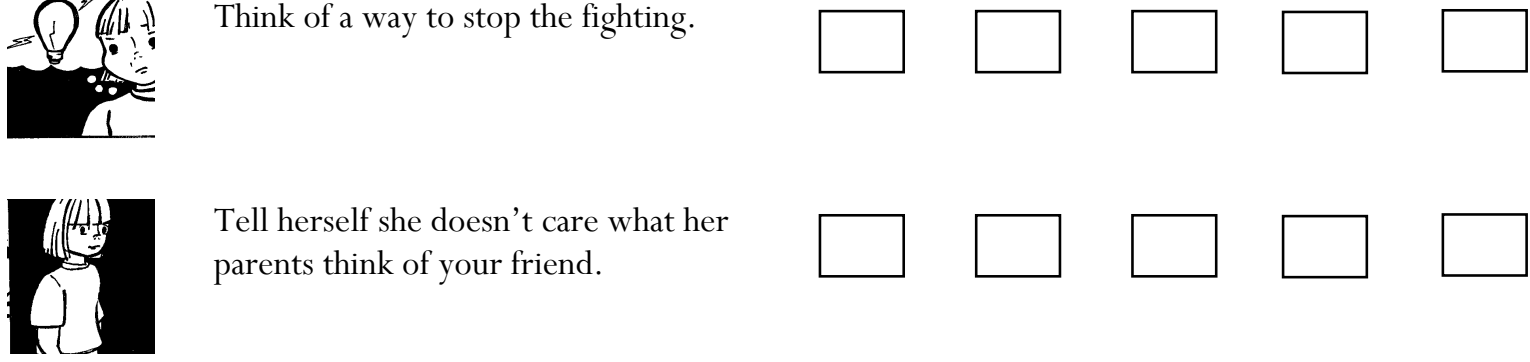

Tell herself she doesn't care what her parents think of your friend.
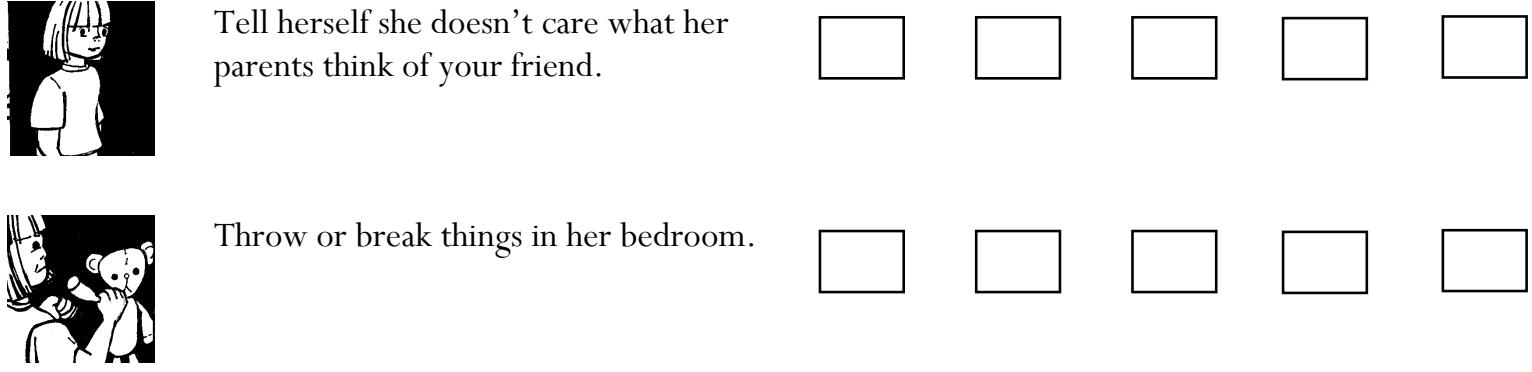

Throw or break things in her bedroom.
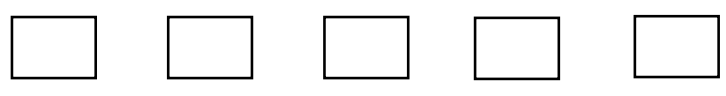
A teacher is picking on your child at school.

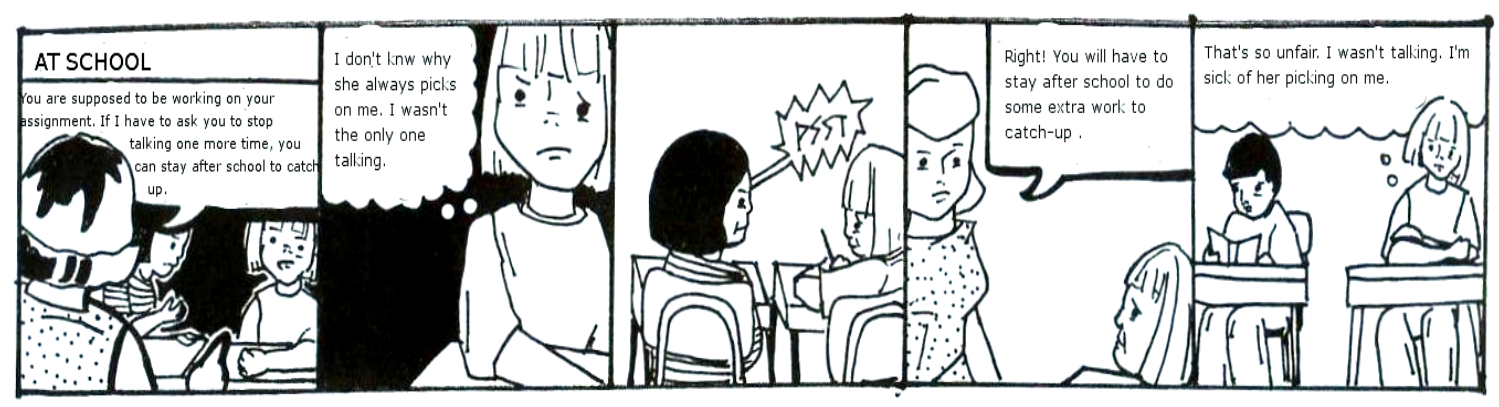

What would you recommend she do?

$\begin{array}{cccc}\text { Strongly } & \text { Somewhat } & \text { Neutral } & \begin{array}{c}\text { Somewhat Strongly } \\ \text { encourage encourage }\end{array}\end{array}$

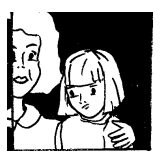

Talk to someone she trusts about how unfair she thinks things are at school.
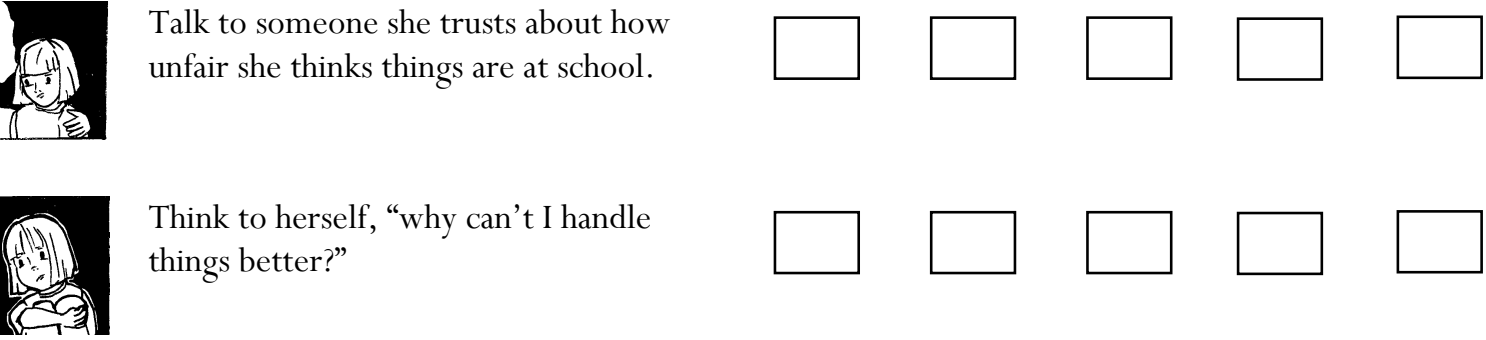

Think to herself, "why can't I handle things better?"
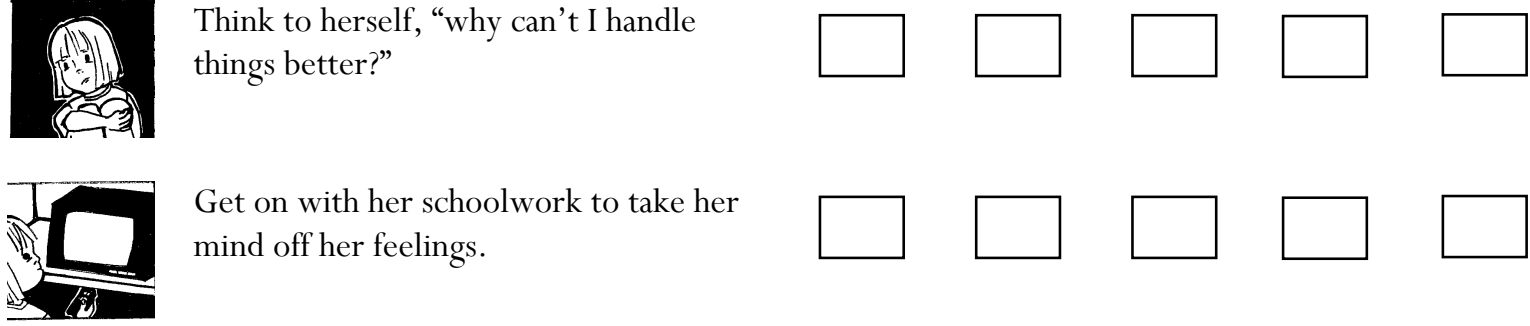

Get on with her schoolwork to take her mind off her feelings.
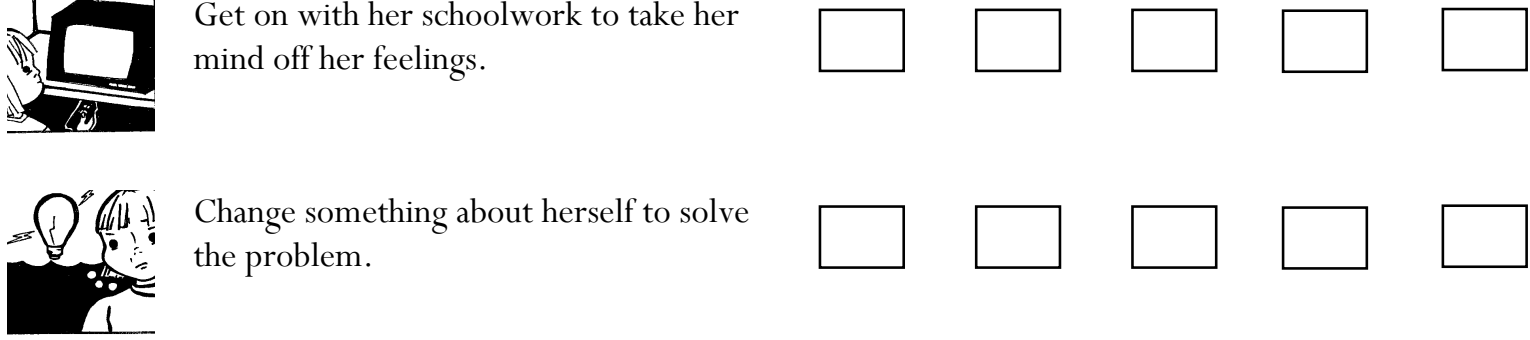

Change something about herself to solve the problem.
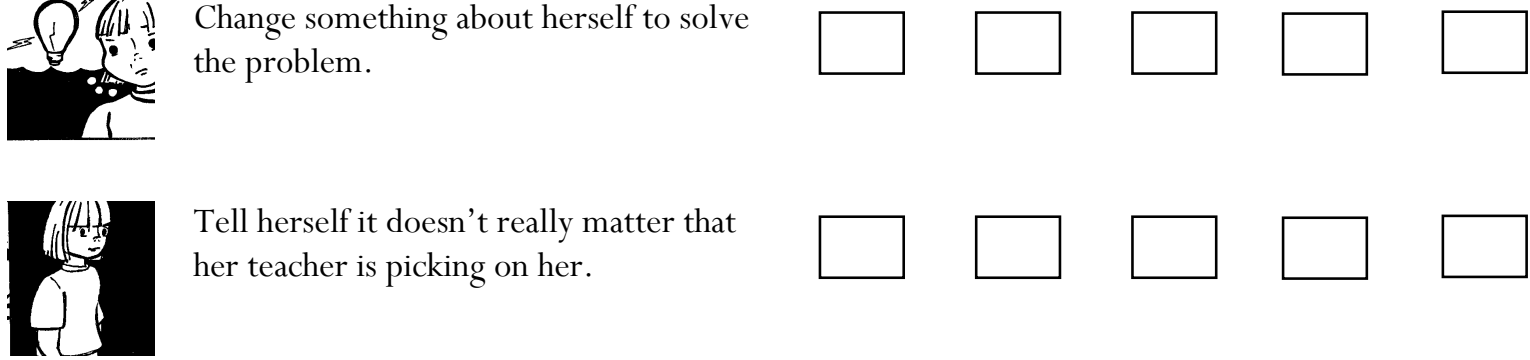

Tell herself it doesn't really matter that her teacher is picking on her.
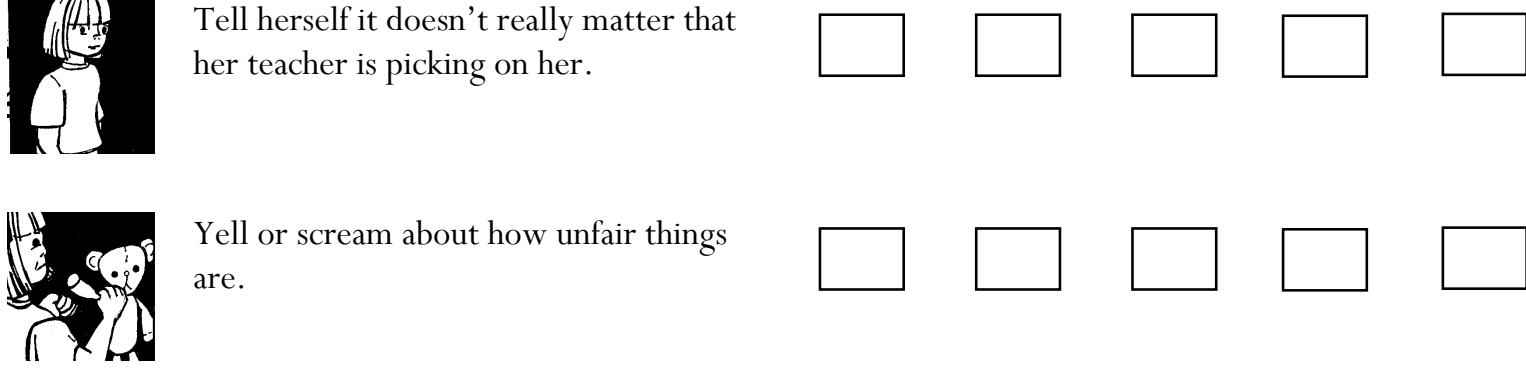

Yell or scream about how unfair things are.
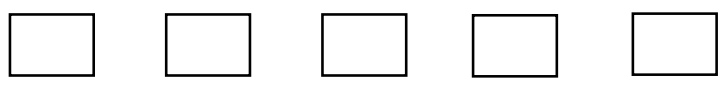
Your child is being teased at school by other children.

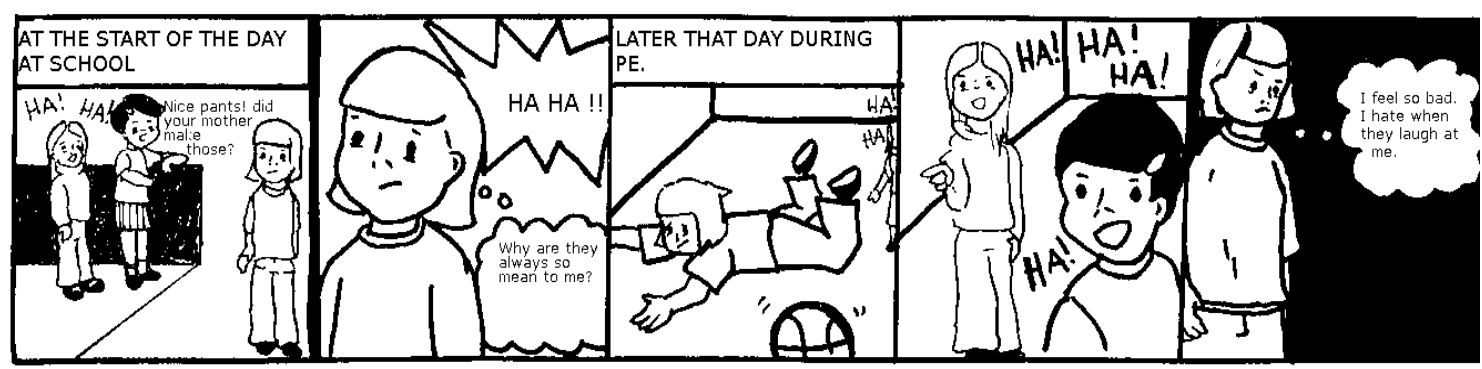

What would you recommend she do?

Strongly Somewhat Neutral Somewhat Strongly discourage discourage encourage encourage

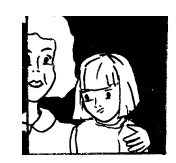

Talk to someone she trusts about the other kids teasing her.
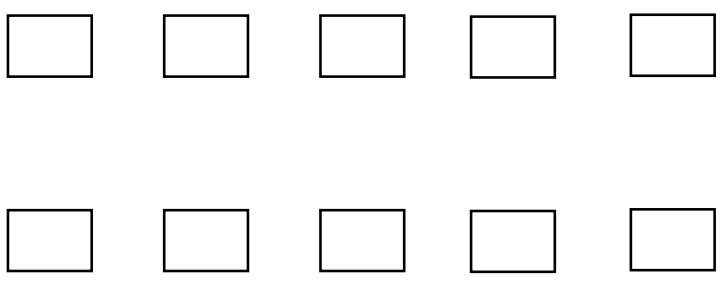
mood."

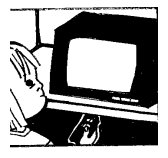

Play a game that she enjoys to take her mind off her feelings.
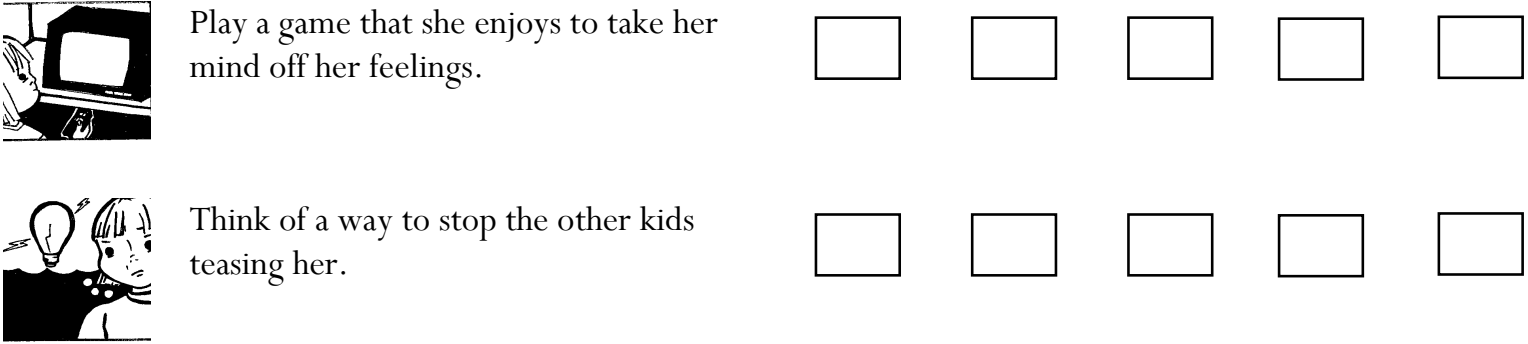

Think of a way to stop the other kids teasing her.
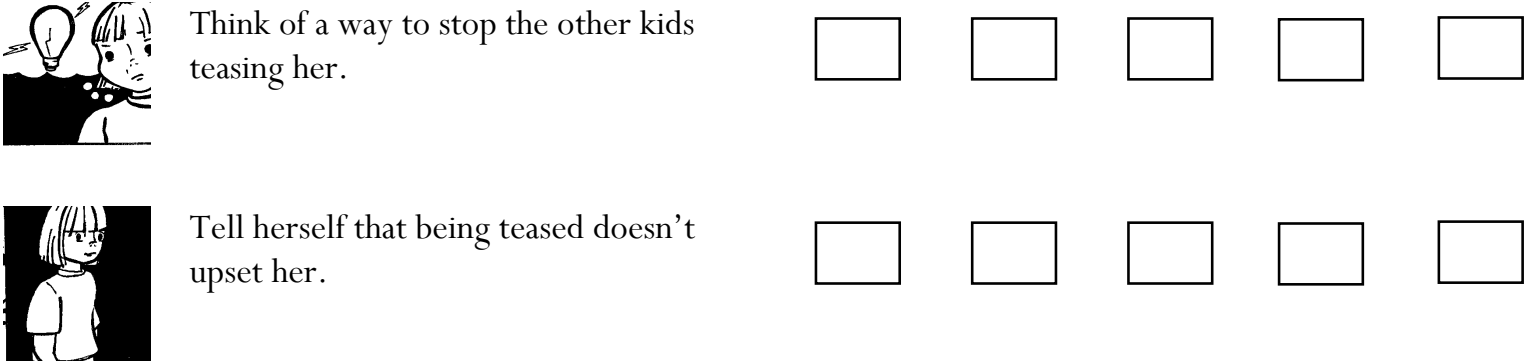

Tell herself that being teased doesn't upset her.
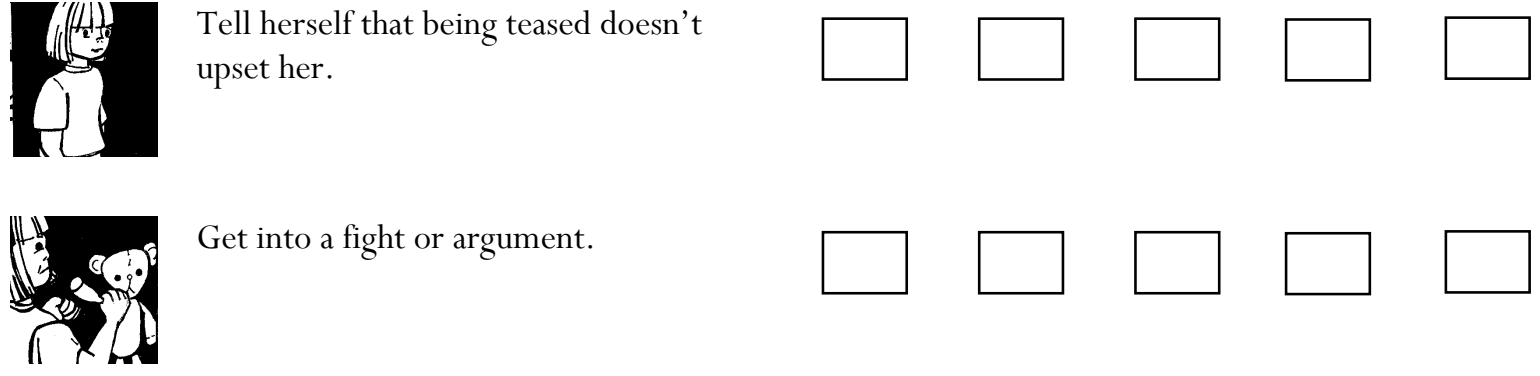

Get into a fight or argument. 
Your child is not invited to a birthday party that they wanted to go to.

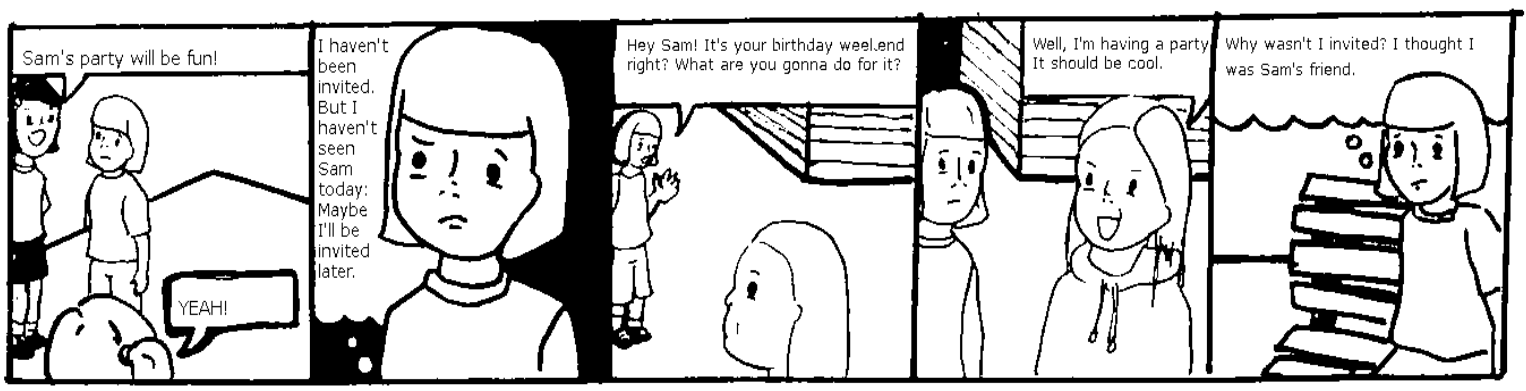

What would you recommend she do?

$\begin{array}{cccc}\begin{array}{c}\text { Strongly } \\ \text { discourage }\end{array} & \begin{array}{c}\text { Somewhat } \\ \text { discourage }\end{array} & \text { Neutral } & \begin{array}{c}\text { Somewhat Strongly } \\ \text { encourage encourage }\end{array}\end{array}$

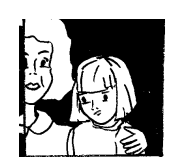

Talk to somebody about not being invited to the party.
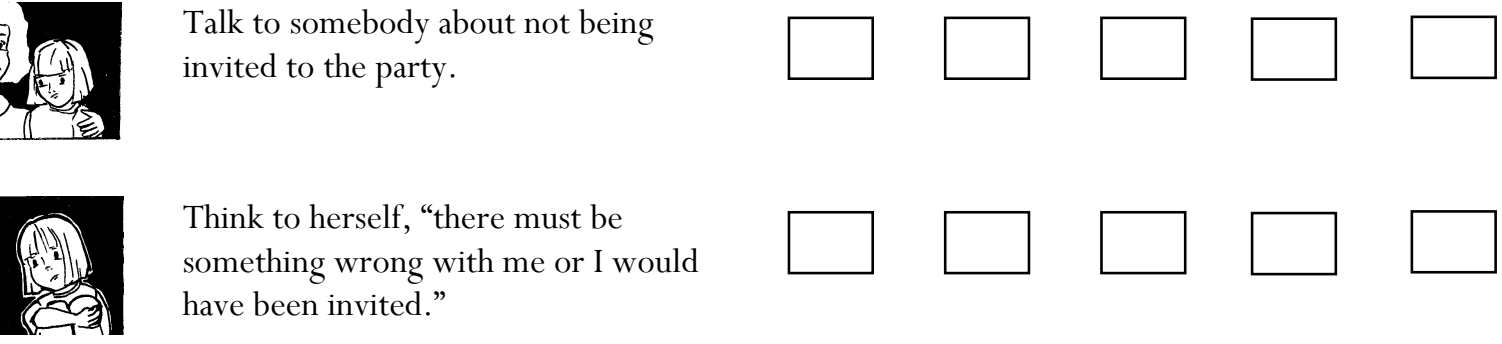

Think to herself, "there must be something wrong with me or I would have been invited."
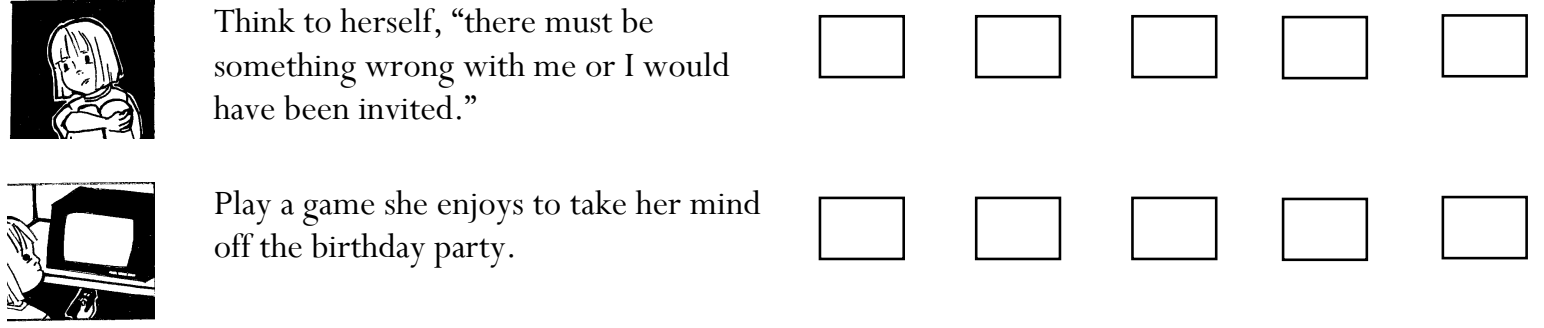

Play a game she enjoys to take her mind off the birthday party.
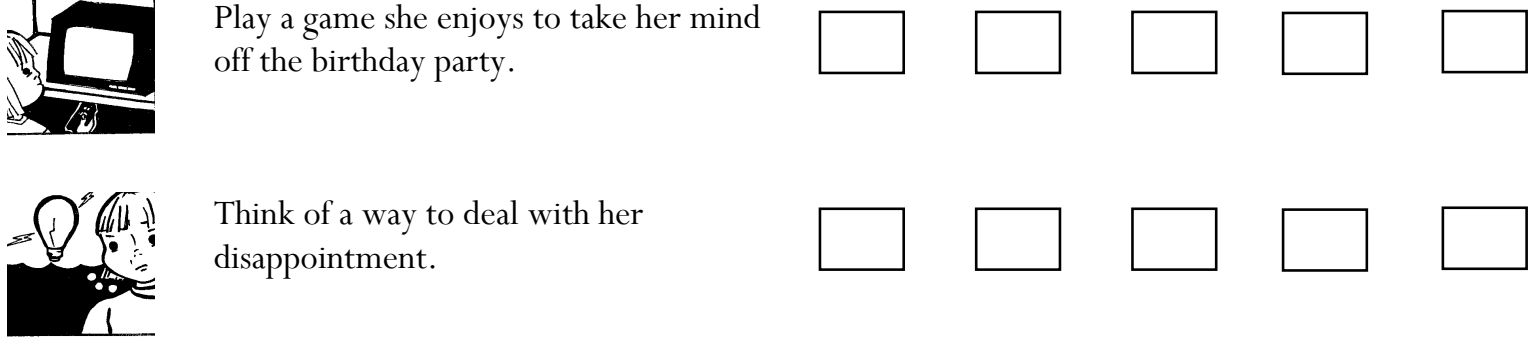

Think of a way to deal with her disappointment.
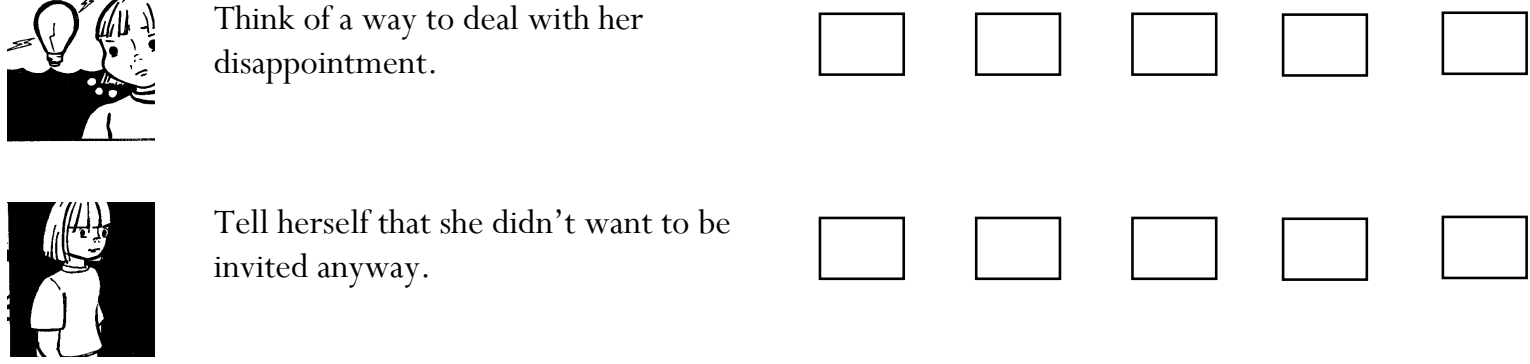

Tell herself that she didn't want to be invited anyway.
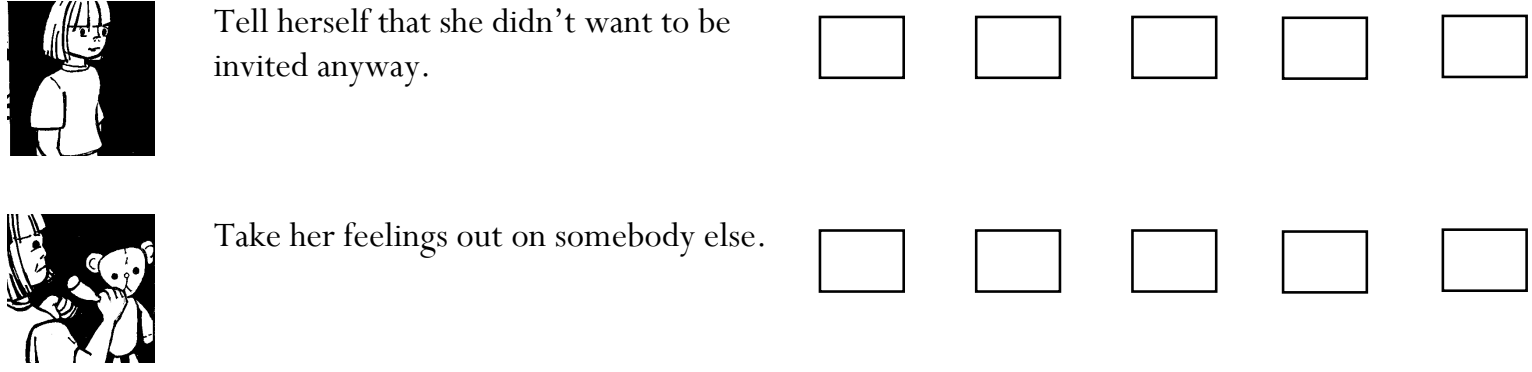

Take her feelings out on somebody else.
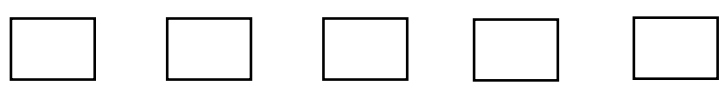
Your child is bored at school by the work.

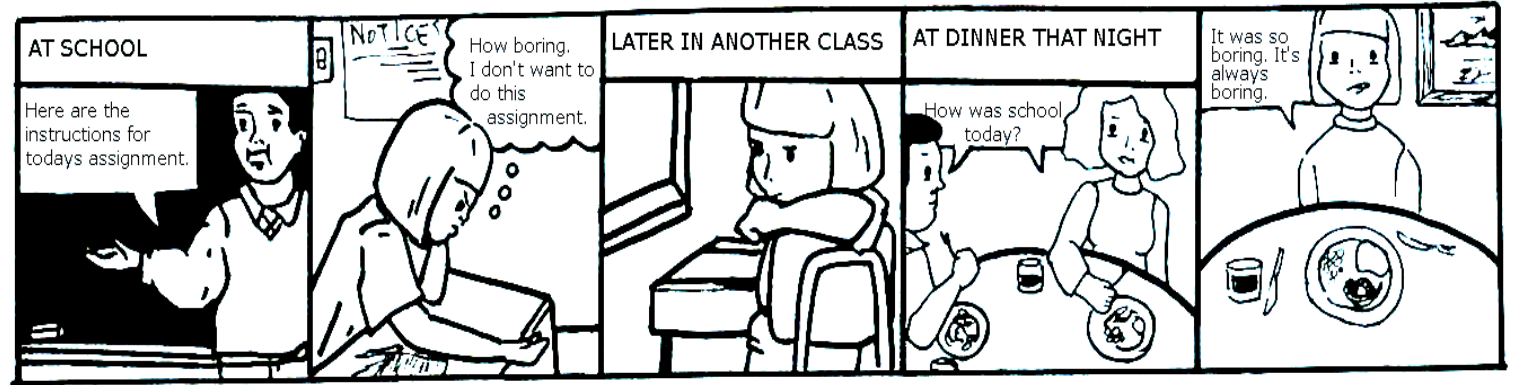

What would you recommend she do?

$\begin{array}{cccc}\begin{array}{c}\text { Strongly } \\ \text { discourage }\end{array} & \begin{array}{c}\text { Somewhat } \\ \text { discourage }\end{array} & \text { Neutral } & \begin{array}{c}\text { Somewhat Strongly } \\ \text { encourage encourage }\end{array}\end{array}$

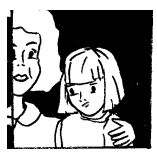

Talk to her parents about how bored

she is at school.
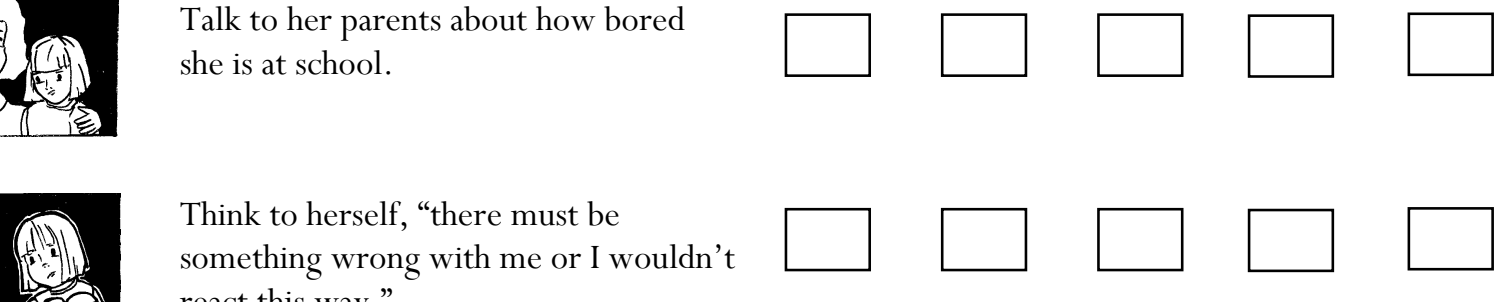

Think to herself, "there must be

something wrong with me or I wouldn't
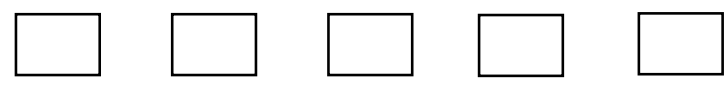

react this way."

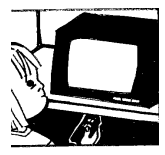

Do something she enjoys to take her

mind off school.
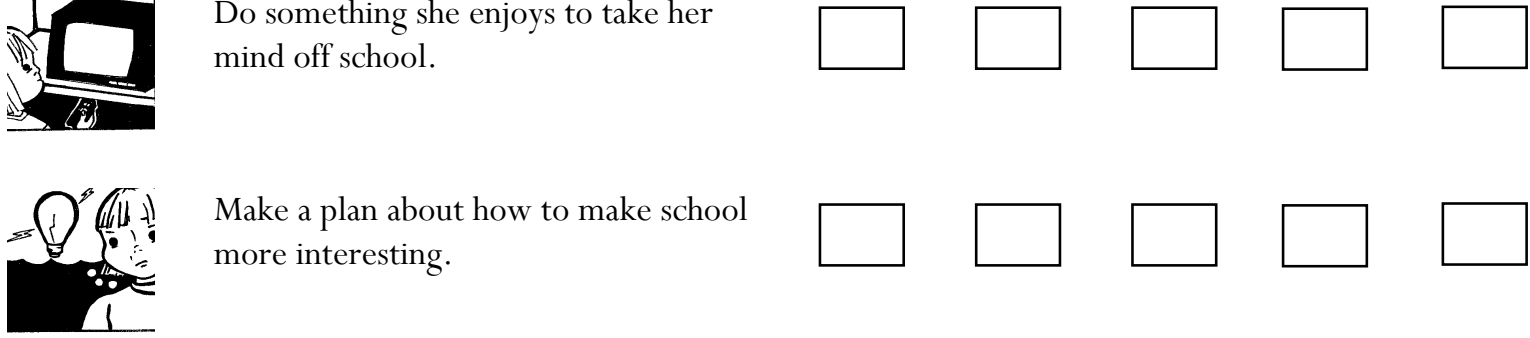

Make a plan about how to make school more interesting.
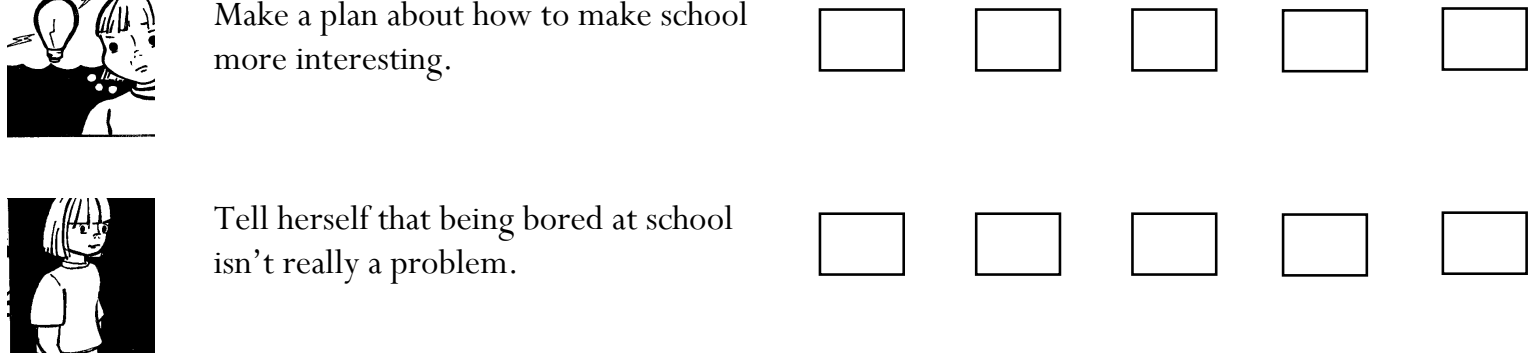

Tell herself that being bored at school isn't really a problem.
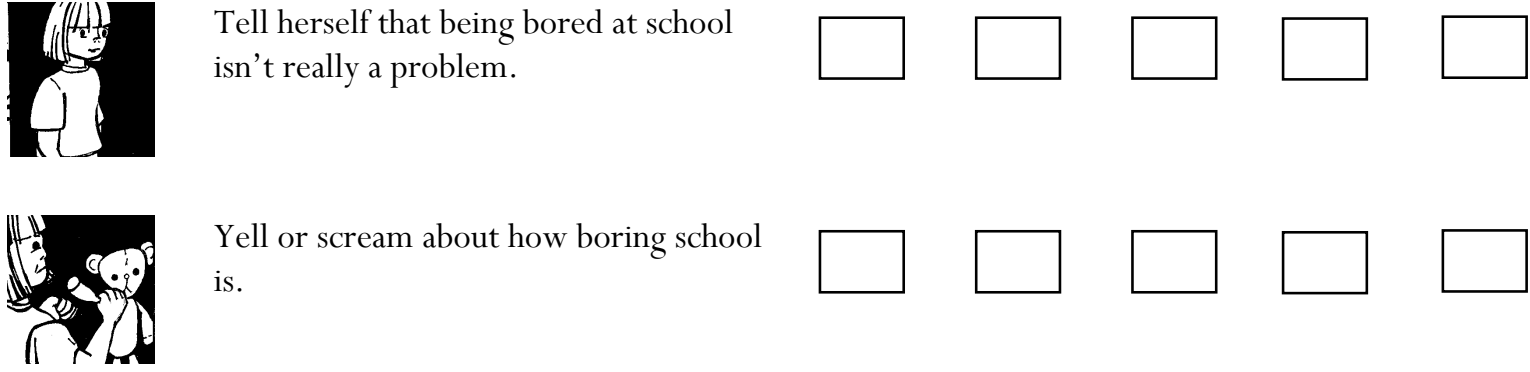

Yell or scream about how boring school is.
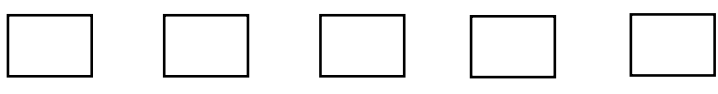
Your child does badly in a test that they studied hard for.

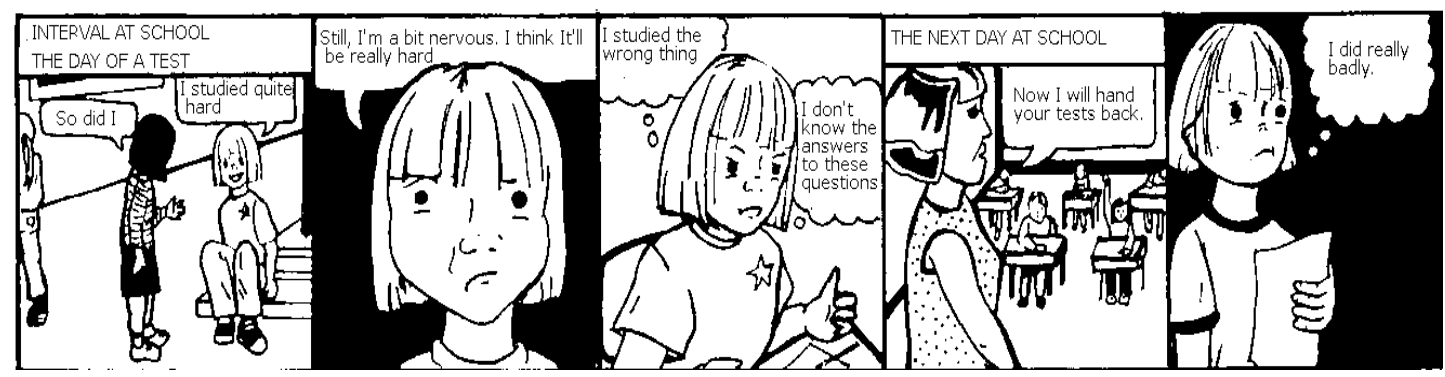

What would you recommend she do? $\begin{array}{clll}\text { Strongly } & \text { Somewhat } & \text { Neutral } & \begin{array}{c}\text { Somewhat Strongly } \\ \text { encourage encourage }\end{array}\end{array}$
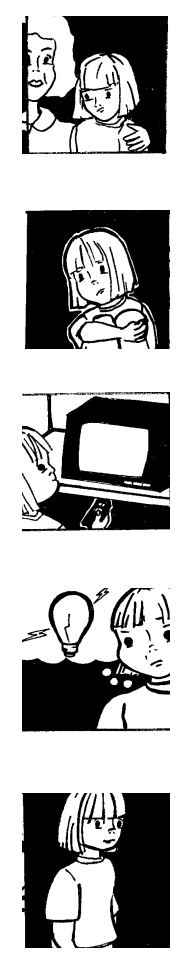

Tell herself that the test didn't really matter anyway.

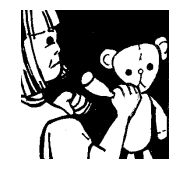
feelings.

Think to herself, "I won't be able to off her feelings.

Make a plan to ensure she is better prepared for her next test.

Throw things or break things.
Talk to somebody she trusts about her concentrate if I keep feeling this way."

Play a game she likes, to take her mind
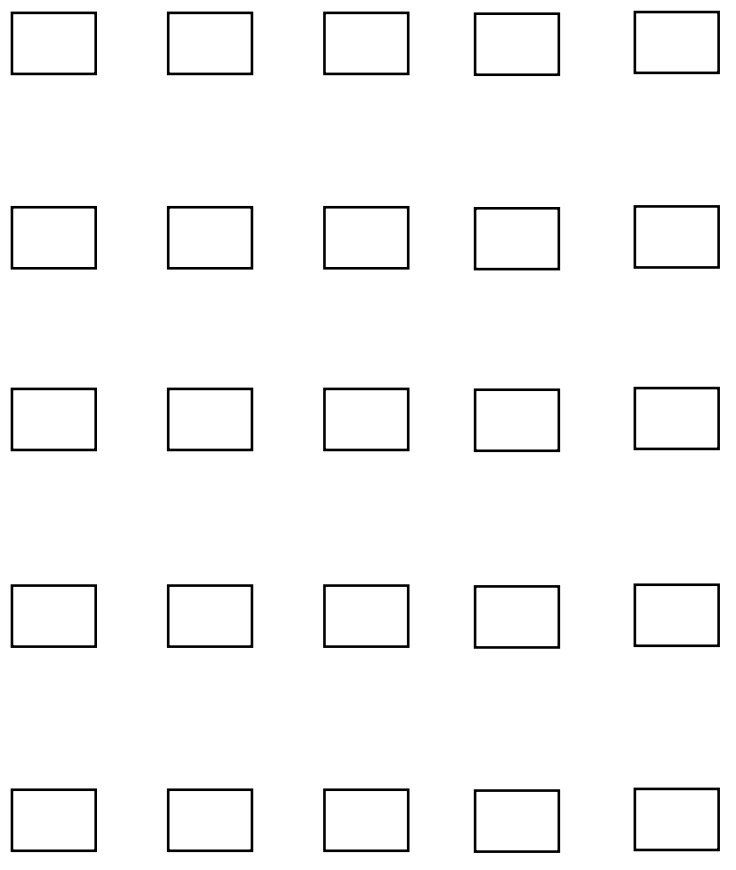
Your child is blamed by their sibling for something they didn't do.

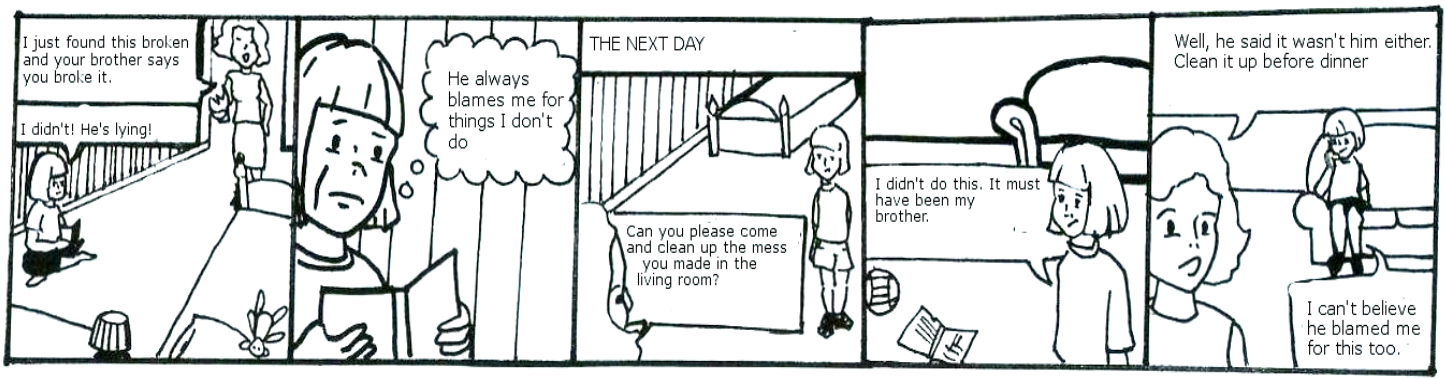

What would you recommend she do?

$\begin{array}{cccc}\begin{array}{c}\text { Strongly } \\ \text { discourage }\end{array} & \begin{array}{c}\text { Somewhat } \\ \text { discourage }\end{array} & \text { Neutral } & \begin{array}{c}\text { Somewhat Strongly } \\ \text { encourage encourage }\end{array}\end{array}$

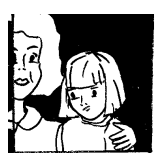

Talk to her mother about how she is feeling.
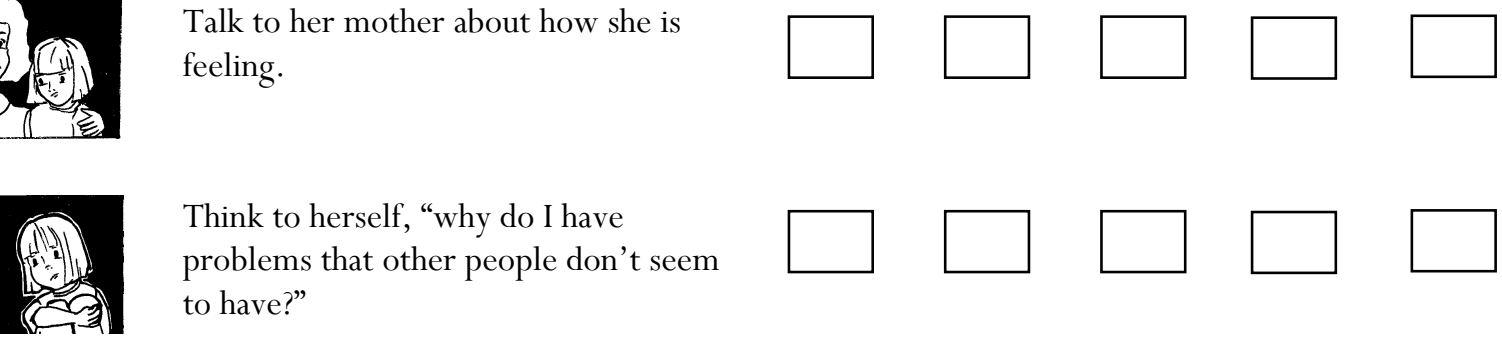

Think to herself, "why do I have problems that other people don't seem to have?"

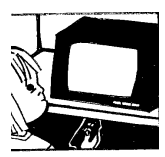

Go back to reading her book to make herself feel better.
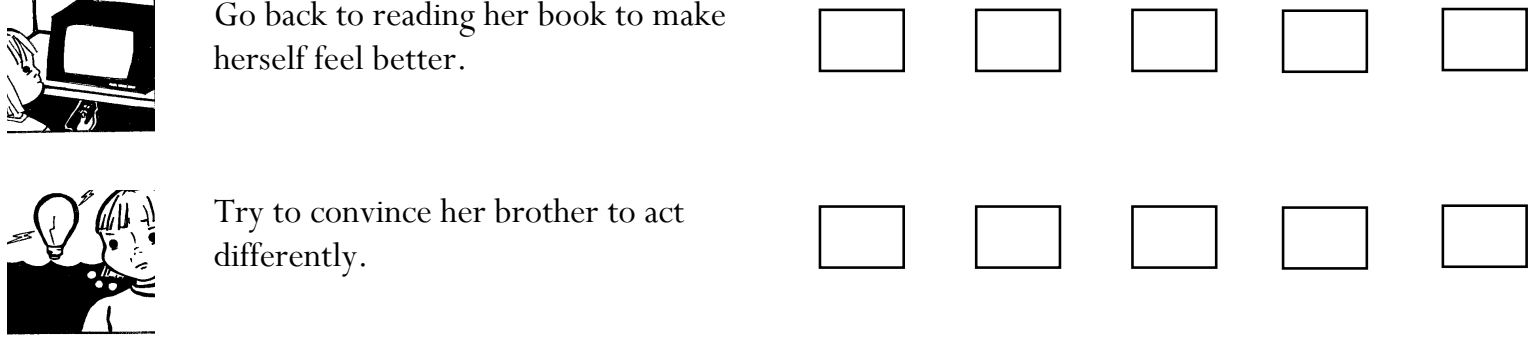

Try to convince her brother to act differently.
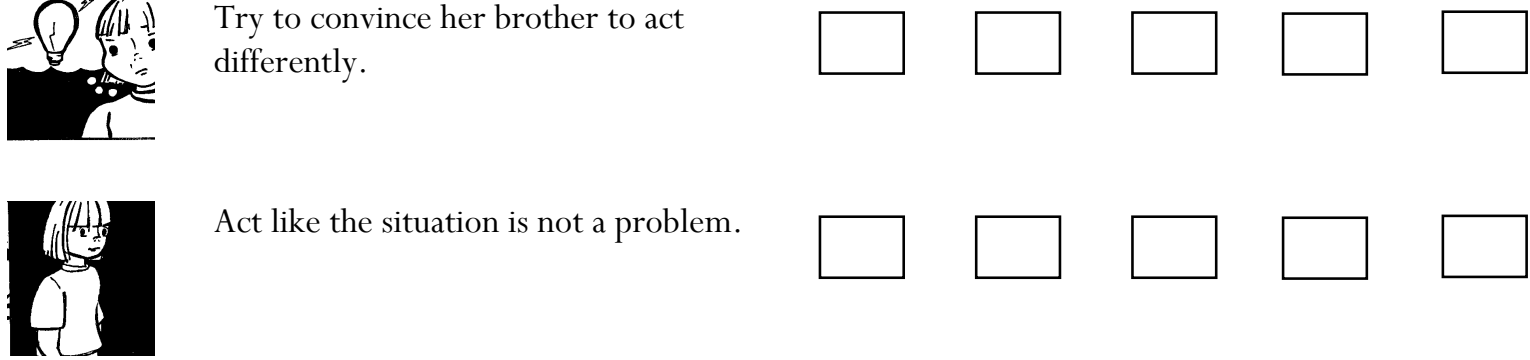

Act like the situation is not a problem.
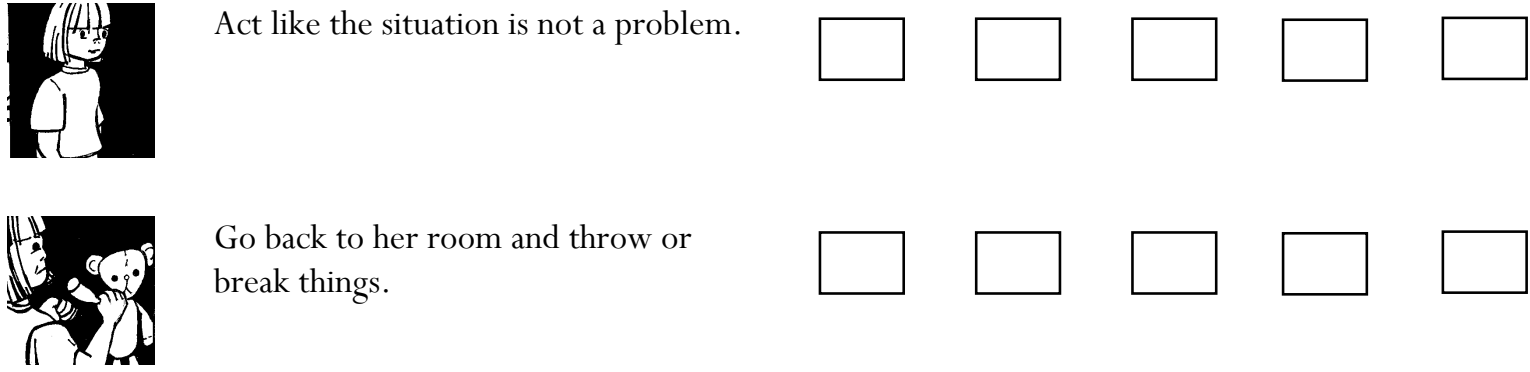

Go back to her room and throw or break things.
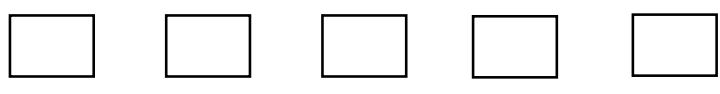


\section{Appendix G: Perception of False Self Scale}

\begin{tabular}{|c|c|c|c|c|c|c|}
\hline & & $\begin{array}{l}\text { Strongly } \\
\text { disagree }\end{array}$ & & Neutral & & $\begin{array}{l}\text { trongly } \\
\text { agree }\end{array}$ \\
\hline $1 . *$ & $\begin{array}{l}\text { I say what I think even if it is different from the } \\
\text { opinions of others }\end{array}$ & 1 & 2 & 3 & 4 & 5 \\
\hline $2 . *$ & I cannot express my opinions to others & 1 & 2 & 3 & 4 & 5 \\
\hline 3. & $\begin{array}{l}\text { I prefer to say nothing if my opinion differs from } \\
\text { other people }\end{array}$ & 1 & 2 & 3 & 4 & 5 \\
\hline 4. & The way I dress reflects my own style & 1 & 2 & 3 & 4 & 5 \\
\hline $5 . *$ & I act one way, but want to act a different way & 1 & 2 & 3 & 4 & 5 \\
\hline 6. & $\begin{array}{l}\text { To gain approval from others I need to act in a } \\
\text { specific way }\end{array}$ & 1 & 2 & 3 & 4 & 5 \\
\hline 7. & I always say what I mean no matter what & 1 & 2 & 3 & 4 & 5 \\
\hline $8 . *$ & I don't let people see the real me & 1 & 2 & 3 & 4 & 5 \\
\hline 9. & I judge myself by how I think other people see me & 1 & 2 & 3 & 4 & 5 \\
\hline 10. & $\begin{array}{l}\text { Even if I am sad or angry on the inside, I try to look } \\
\text { happy on the outside. }\end{array}$ & 1 & 2 & 3 & 4 & 5 \\
\hline 11. & I appear to others the way I am & 1 & 2 & 3 & 4 & 5 \\
\hline 12. & I say things that I think other people want to hear & 1 & 2 & 3 & 4 & 5 \\
\hline $13 . *$ & My thoughts are not important to others & 1 & 2 & 3 & 4 & 5 \\
\hline 14.* & I hide the real me by looking like others & 1 & 2 & 3 & 4 & 5 \\
\hline $15 *^{*}$ & I act in ways that express who I really am & 1 & 2 & 3 & 4 & 5 \\
\hline 16. & If people saw the real me, they would not like me & 1 & 2 & 3 & 4 & 5 \\
\hline 17. & $\begin{array}{l}\text { When making decisions, the opinions of others } \\
\text { matter more than mine }\end{array}$ & 1 & 2 & 3 & 4 & 5 \\
\hline 18.* & I hide my true feelings if I think they will upset others & 1 & 2 & 3 & 4 & 5 \\
\hline 19. & $\begin{array}{l}\text { I ask people close to me their opinion about how I } \\
\text { look }\end{array}$ & 1 & 2 & 3 & 4 & 5 \\
\hline 20. & $\begin{array}{l}\text { What I say on the outside is different to what I think } \\
\text { on the inside }\end{array}$ & 1 & 2 & 3 & 4 & 5 \\
\hline 21. & I am sure that my real opinions matter to others & 1 & 2 & 3 & 4 & 5 \\
\hline 22. & $\begin{array}{l}\text { I hide what I really think if it is different from other } \\
\text { people's views }\end{array}$ & 1 & 2 & 3 & 4 & 5 \\
\hline $23 . *$ & I tend to say one thing even when I think another & 1 & 2 & 3 & 4 & 5 \\
\hline 24. & I change my appearance if I think others do not like it & 1 & 2 & 3 & 4 & 5 \\
\hline 25. & How other people see me is important to me & 1 & 2 & 3 & 4 & 5 \\
\hline 26. & I present myself to others as I truly am & 1 & 2 & 3 & 4 & 5 \\
\hline 27. & I like to dress the same as my friends & 1 & 2 & 3 & 4 & 5 \\
\hline 28. & I don't feel responsible for other people's feelings & 1 & 2 & 3 & 4 & 5 \\
\hline 29. & I can express different opinions to other peoples & 1 & 2 & 3 & 4 & 5 \\
\hline $30 * *$ & I can talk openly to others about my feelings & 1 & 2 & 3 & 4 & 5 \\
\hline
\end{tabular}


31.* I stay quiet when I don't agree with other people $\begin{array}{llllllll}1 & 2 & 3 & 4 & 5\end{array}$

32.* I don't like to look different from other people $\begin{array}{lllllll}1 & 2 & 3 & 4 & 5\end{array}$

33. Others don't see the real me $\quad \begin{array}{llllll}1 & 2 & 3 & 4 & 5\end{array}$

34. I spend a lot of time thinking about how other people $\begin{array}{llllll}1 & 2 & 3 & 4 & 5\end{array}$

feel

35. I act in ways that are not different to who I truly am $\quad \begin{array}{llllll}1 & 2 & 3 & 4 & 5\end{array}$

36. The way I look reflects the real me $\quad \begin{array}{llllll}1 & 2 & 3 & 4 & 5\end{array}$

37.* If people knew what I was really like on the inside, $\begin{array}{lllllll}1 & 2 & 3 & 4 & 5\end{array}$ they would not like me

38.* Other people's feelings are more important than $\quad \begin{array}{lllllll}1 & 2 & 3 & 4 & 5\end{array}$ mine

39. My point of view is not respected by others $\quad \begin{array}{lllllll}1 & 2 & 3 & 4 & 5\end{array}$

40. I say what I think even if it will cause an argument $\quad \begin{array}{llllll}1 & 2 & 3 & 4 & 5\end{array}$

41.* I spend a lot of time thinking about how other people $\begin{array}{llllll}1 & 2 & 3 & 4 & 5\end{array}$ see me

42. I look the way I like, not the way other people want $\begin{array}{llllll}1 & 2 & 3 & 4 & 5\end{array}$

me to look

43.* What I say on the outside is different to what I think $\begin{array}{lllllll}1 & 2 & 3 & 4 & 5\end{array}$ on the inside

Note: $*=$ items in the final 16 -item POFS scale. 


\section{Appendix H: The Revised Manifest Anxiety Scale for Children}

Please read each question carefully. Put a circle around the word "yes" if you think it is true about you. Put a circle around the word "no" if you think it is not true about you. Please be as honest as you can in answering these questions.

1. I have trouble making up my mind.

Yes $\quad$ No

2. I get nervous when things do not go the right way for me.

Yes No

3. Others seem to do things easier than I can.

Yes No

4. I often have trouble getting my breath.

Yes No

5. I worry a lot of the time.

Yes No

6. I am afraid a lot of the time.

Yes No

7. I get mad easily.

Yes No

8. I worry about what my parents will say to me.

Yes No

9. I feel that others do not like the way I do things.

Yes No

10. It is hard for me to get to sleep at night.

Yes No

11. I worry about what other people will think of me.

Yes No

12. I feel alone even when there are people with me.

Yes No

13. I often feel sick in my stomach.

Yes No

14. My feelings get hurt easily.

Yes No

15. My hands feel sweaty.

Yes No

16. I am tired a lot.

Yes

No

17. I worry about what is going to happen.

Yes No

18. Other people are happier than me.

Yes No

19. I have bad dreams.

Yes No

20. My feelings get hurt easily when I am fussed at

Yes No

21. I feel someone will tell me I do things the wrong way.

Yes

No

22. I wake up scared some of the time.

Yes No

23. I worry when I go to bed at night.

Yes No

24. It is hard for me to keep my mind on my school work.

Yes No

25. I wriggle in my seat a lot.

Yes No

26. I am nervous.

Yes

No

27. A lot of people are against me.

Yes No

28. I often worry about something bad happening to me.

Yes $\quad$ No 


\section{Appendix I: The Say What I Think Around Others Scale}

For the following scale, select which statement best describes you on each line. Then indicate whether that statement is "really true of you" or "sort of true of you". For each pair of statements, only tick one box. Remember, there are no right or wrong answers, indicate how you think you are, not how you SHOULD be.

\begin{tabular}{|c|c|c|c|c|c|c|}
\hline $\begin{array}{c}\text { Really } \\
\text { true }\end{array}$ & $\begin{array}{c}\text { Sort of } \\
\text { true }\end{array}$ & & & & $\begin{array}{c}\text { Sort of } \\
\text { true }\end{array}$ & $\begin{array}{c}\text { Really } \\
\text { True }\end{array}$ \\
\hline & & $\begin{array}{l}\text { Some people share what } \\
\text { they are thinking with } \\
\text { female classmates }\end{array}$ & BUT & $\begin{array}{l}\text { Other people find it } \\
\text { hard to share what they } \\
\text { are really thinking with } \\
\text { female classmates }\end{array}$ & & \\
\hline & & $\begin{array}{l}\text { Some people usually don't } \\
\text { say what's on their mind } \\
\text { around female classmates }\end{array}$ & BUT & $\begin{array}{l}\text { Other people do say } \\
\text { what's on their mind } \\
\text { around female } \\
\text { classmates }\end{array}$ & & \\
\hline & & $\begin{array}{l}\text { Some people are able to } \\
\text { express their opinions to } \\
\text { female classmates }\end{array}$ & BUT & $\begin{array}{l}\text { Other people have } \\
\text { trouble expressing their } \\
\text { opinions to female } \\
\text { classmates }\end{array}$ & & \\
\hline & & $\begin{array}{l}\text { Some people are able to } \\
\text { let female classmates } \\
\text { know what's important to } \\
\text { them }\end{array}$ & BUT & $\begin{array}{l}\text { Other people are not } \\
\text { able to let female } \\
\text { students know what's } \\
\text { important to them }\end{array}$ & & \\
\hline & & $\begin{array}{l}\text { Some people have a hard } \\
\text { time expressing their } \\
\text { point of view to female } \\
\text { classmates }\end{array}$ & BUT & $\begin{array}{l}\text { Other people can } \\
\text { express their point of } \\
\text { view to female } \\
\text { classmates }\end{array}$ & & \\
\hline
\end{tabular}




\section{Appendix J: The Silencing the Self Scale}

Please circle the number that best describes how well each statement describes you.

\begin{tabular}{|c|c|c|c|c|c|c|}
\hline & & $\begin{array}{l}\text { Strongly } \\
\text { disagree }\end{array}$ & & Neutral & & $\begin{array}{c}\text { Strongly } \\
\text { agree }\end{array}$ \\
\hline 1. & $\begin{array}{l}\text { I don't speak my feelings when I know they will } \\
\text { cause disagreement. }\end{array}$ & 1 & 2 & 3 & 4 & 5 \\
\hline 2. & $\begin{array}{l}\text { I tend to judge myself by how I think other } \\
\text { people see me }\end{array}$ & 1 & 2 & 3 & 4 & 5 \\
\hline 3. & $\begin{array}{l}\text { I feel dissatisfied with myself because I should be } \\
\text { able to do all the things people are } \\
\text { supposed to be able to do these days. }\end{array}$ & 1 & 2 & 3 & 4 & 5 \\
\hline 4. & $\begin{array}{l}\text { When another persons needs and feelings conflict } \\
\text { with my own, I always state mine clearly }\end{array}$ & 1 & 2 & 3 & 4 & 5 \\
\hline 5. & $\begin{array}{l}\text { Instead of risking arguments, I would rather not } \\
\text { rock the boat }\end{array}$ & 1 & 2 & 3 & 4 & 5 \\
\hline 6. & $\begin{array}{l}\text { I speak my feelings even when it leads to } \\
\text { problems or disagreements }\end{array}$ & 1 & 2 & 3 & 4 & 5 \\
\hline 7. & $\begin{array}{l}\text { When someone's needs or opinions conflict with } \\
\text { mine, rather than asserting my own } \\
\text { point of view I usually end up agreeing } \\
\text { with him/her }\end{array}$ & 1 & 2 & 3 & 4 & 5 \\
\hline 8. & $\begin{array}{l}\text { When it looks as though certain of my needs cant } \\
\text { be met, I usually realise that they } \\
\text { weren't very important anyway }\end{array}$ & 1 & 2 & 3 & 4 & 5 \\
\hline 9. & $\begin{array}{l}\text { When I make decisions, other people's thoughts } \\
\text { and opinions influence me more than my } \\
\text { own thoughts and opinions }\end{array}$ & 1 & 2 & 3 & 4 & 5 \\
\hline 10. & I rarely express my anger at those close to me & 1 & 2 & 3 & 4 & 5 \\
\hline 11. & $\begin{array}{l}\text { I think it's better to keep my feelings to myself } \\
\text { when they conflict with others }\end{array}$ & 1 & 2 & 3 & 4 & 5 \\
\hline 12. & I feel responsible for other peoples feelings & 1 & 2 & 3 & 4 & 5 \\
\hline 13. & $\begin{array}{l}\text { I find it hard to know what I think and feel } \\
\text { because I spend a lot of time thinking } \\
\text { about how other people are feeling }\end{array}$ & 1 & 2 & 3 & 4 & 5 \\
\hline 14. & $\begin{array}{l}\text { I try to bury my feelings when I think they will } \\
\text { cause trouble with others }\end{array}$ & 1 & 2 & 3 & 4 & 5 \\
\hline 15. & $\begin{array}{l}\text { I never seem to measure up to the standards I set } \\
\text { for myself }\end{array}$ & 1 & 2 & 3 & 4 & 5 \\
\hline
\end{tabular}




\section{Appendix K: Everyday Life Events Scale For Children}

Below is a list of everyday events that can happen to anyone. Please indicate a) whether each event happened, b) and if it happened, how much of a problem it was (by circling a number). Report events if they happened in the last month only.

\begin{tabular}{|c|c|c|c|c|c|c|}
\hline \multirow{2}{*}{\multicolumn{3}{|c|}{ Has this event happened to you in the last month? }} & \multicolumn{4}{|c|}{ Was the event a problem? } \\
\hline & & & No & A little & Some & A lot \\
\hline 1. You misplaced or lost something & Yes & No & 0 & 1 & 2 & 3 \\
\hline 2. Someone teased you or made fun of you & Yes & No & 0 & 1 & 2 & 3 \\
\hline 3. Not weighing enough & Yes & No & 0 & 1 & 2 & 3 \\
\hline 4. Someone in your family was sick & Yes & No & 0 & 1 & 2 & 3 \\
\hline 5. You didn't have enough money & Yes & No & 0 & 1 & 2 & 3 \\
\hline 6. Schoolwork was boring & Yes & No & 0 & 1 & 2 & 3 \\
\hline $\begin{array}{l}\text { 7. You were rushed, you couldn't relax or take } \\
\text { it easy }\end{array}$ & Yes & No & 0 & 1 & 2 & 3 \\
\hline 8. You were sick & Yes & No & 0 & 1 & 2 & 3 \\
\hline 9. Doing housework or cleaning & Yes & No & 0 & 1 & 2 & 3 \\
\hline 10. Difficulty in finding a quiet place at home & Yes & No & 0 & 1 & 2 & 3 \\
\hline $\begin{array}{l}\text { 11. You were bored, not enough fun things to } \\
\text { do }\end{array}$ & Yes & No & 1 & 2 & 3 & \\
\hline $\begin{array}{l}\text { 12. Difficult to find the time to study and do } \\
\text { assignments }\end{array}$ & Yes & No & 0 & 1 & 2 & 3 \\
\hline $\begin{array}{l}\text { 13. You did something foolish or embarrassing } \\
\text { in front of others }\end{array}$ & Yes & No & 0 & 1 & 2 & 3 \\
\hline 14. You were avoided by someone & Yes & No & 0 & 1 & 2 & 3 \\
\hline 15. Classmate(s) was/were mean to me & Yes & No & 0 & 1 & 2 & 3 \\
\hline 16. You went to the doctor or dentist & Yes & No & 0 & 1 & 2 & 3 \\
\hline 17. Your mum or dad were mean to you & Yes & No & 0 & 1 & 2 & 3 \\
\hline $\begin{array}{l}\text { 18. Your mum or dad told you about their } \\
\text { worries }\end{array}$ & Yes & No & 0 & 1 & 2 & 3 \\
\hline 19. Your body changed shape & Yes & No & 0 & 1 & 2 & 3 \\
\hline $\begin{array}{l}\text { 20. You were unable to talk to other people } \\
\text { about your feelings }\end{array}$ & Yes & No & 0 & 1 & 2 & 3 \\
\hline 21. You don't like the way you look & Yes & No & 0 & 1 & 2 & 3 \\
\hline 22. Being pressured to drink or use drugs & Yes & No & 0 & 1 & 2 & 3 \\
\hline 23. Misunderstanding with a friend & Yes & No & 0 & 1 & 2 & 3 \\
\hline 24. Not getting enough sleep & Yes & No & 0 & 1 & 2 & 3 \\
\hline 25. You don't look the way you want to. & Yes & No & 0 & 1 & 2 & 3 \\
\hline $\begin{array}{l}\text { 26. Schoolwork was more difficult than } \\
\text { expected }\end{array}$ & Yes & No & 0 & 1 & 2 & 3 \\
\hline $\begin{array}{l}\text { 27. Not being with your family as much as you } \\
\text { wanted }\end{array}$ & Yes & No & 0 & 1 & 2 & 3 \\
\hline
\end{tabular}


28. Food that you're eating doesn't taste good

29. Couldn't buy something due to lack of money

30. Someone close to you didn't keep a promise

31. Misunderstanding with your boy/girlfriend

32. Don't like where you are living

33. Difficult to get to school

34. You saw a family member who was drunk

35. Too many people live in your house

36. Received an unfair grade/mark for an assignment

37. Being alone too much

38. Weighing too much

39. Not enough time for relaxation

40. Someone has stolen something that belongs to you

\begin{tabular}{ccccc} 
Yes No & 0 & 1 & 2 & 3 \\
Yes No & 0 & 1 & 2 & 3 \\
Yes No & 0 & 1 & 2 & 3 \\
Yes No & 0 & 1 & 2 & 3 \\
Yes No & 0 & 1 & 2 & 3 \\
Yes No & 0 & 1 & 2 & 3 \\
Yes No & 0 & 1 & 2 & 3 \\
Yes No & 0 & 1 & 2 & 3 \\
Yes No & 0 & 1 & 2 & 3 \\
Yes No & 0 & 1 & 2 & 3 \\
Yes No & 0 & 1 & 2 & 3 \\
Yes No & 0 & 1 & 2 & 3 \\
Yes No & 0 & 1 & 2 & 3 \\
\hline
\end{tabular}




\section{Appendix L: Beck Anxiety Inventory}

Please carefully read each item in the list. Indicate how much you have been bothered by each statement during the past month, including today, by circling the number in the corresponding space in the column next to each symptoms.

\begin{tabular}{lcccc}
\hline & Not at all & $\begin{array}{c}\text { Mildly but it } \\
\text { didn't bother } \\
\text { me much }\end{array}$ & $\begin{array}{c}\text { Moderately, it } \\
\text { wasn't pleasant } \\
\text { at times }\end{array}$ & $\begin{array}{c}\text { Severely, it } \\
\text { bothered me a } \\
\text { lot }\end{array}$ \\
\hline 1. Numbness or tingling & 0 & 1 & 2 & 3 \\
2. Feeling hot & 0 & 1 & 2 & 3 \\
3. Wobbliness in legs & 0 & 1 & 2 & 3 \\
4. Unable to relax & 0 & 1 & 2 & 3 \\
5. Fear of worse happening & 0 & 1 & 2 & 3 \\
6. Dizzy or light-headed & 0 & 1 & 2 & 3 \\
7. Heart pounding/racing & 0 & 1 & 2 & 3 \\
8. Unsteady & 0 & 1 & 2 & 3 \\
9. Terrified or afraid & 0 & 1 & 2 & 3 \\
10. Nervous & 0 & 1 & 2 & 3 \\
11. Feeling of choking & 0 & 1 & 2 & 3 \\
12. Hands trembling & 0 & 1 & 2 & 3 \\
13. Shaky / unsteady & 0 & 1 & 2 & 3 \\
14. Fear of losing control & 0 & 1 & 2 & 3 \\
15.Difficulty in breathing & 0 & 1 & 2 & 3 \\
16. Fear of dying & 0 & 1 & 2 & 3 \\
17. Scared & 0 & 1 & 2 & 3 \\
18. Indigestion & 0 & 1 & 2 & 3 \\
19. Faint / light-headed & 0 & 1 & 2 & 3 \\
20. Face flushed & 0 & 1 & 2 & 3 \\
21. Hot/ cold sweats & 0 & 1 & 2 & 3 \\
\hline & 0 & 1 & 2 & 3 \\
\hline
\end{tabular}

